\title{
Singularidades de curvas irredutíveis planas ${ }^{1}$
}

\author{
Grazielle Feliciani Barbosa
}

Orientadora: Profa. Dra. Roberta Godoi Wik Atique

Dissertação apresentada ao Instituto de Ciências Matemáticas e de Computação da Universidade de São Paulo, como parte dos requisitos para obtenção do título de Mestre em Ciências - Área: Matemática.

USP - São Carlos

Fevereiro/2004

\footnotetext{
${ }^{1}$ Este trabalho teve suporte financeiro da Fapesp proc: 01/12535-9
} 


\section{A Comissão Julgadora:}

Profa. Dra. Roberta Godoi Wik Atique

Prof. Dr. Daniel Levcovitz

Profa. Dra. Neuza Kazuko Kakuta

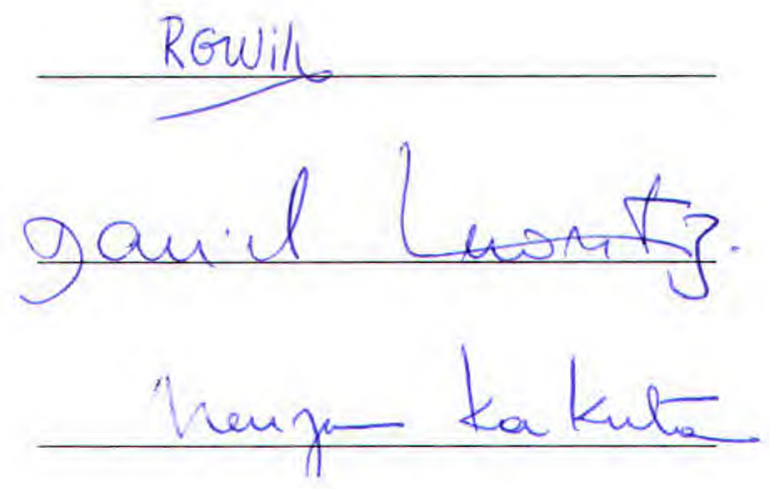


Aos meu pais, à minha irmã Giselle e ao Mauricio com imenso amor e admiração... 


\section{Agradecimentos}

Acima de tudo agradeço a Deus, pela presença constante em minha vida, e por me dar saúde e força em todos as momentos.

Agradeço a Prof. Roberta, pela orientação em todas as etapas da minha formação, pela paciência e principalmente pela amizade.

Que importância tem a família em nossas vidas, nosso refúgio e fortaleza. Agradeço aos meus pais, José Lourenço e Inês, por todo amor e carinho que me dedicam, por terem me ensinado o caminho a seguir e por acreditarem em mim. À minha irmã Giselle, agradeço pela amizade e compreensão. Agradeço à toda minha família, pelas orações que me dispensaram e por terem colaborado muito para que esta etapa fosse concluída. Amo vocês!

Ao meu namorado Mauricio, agradeço por me incentivar a seguir em frente e estar sempre ao meu lado com toda sua dedicação, carinho e amor.

Aos meus amigos de graduação(Mariana, Ronaldinho, Luci, Aline, Érica, Flávia, Fábio, Alex e Kelly), de pós-graduação(Elenice, Ana Carla, Márcio, Olivâine e Rodrigo) e aos fofinhos Carol, Luís Felipe e Pedro que colaboraram para tornar essa caminhada muito mais agradável. Agradeço por todas as alegrias, conversas, ajudas e principalmente pela amizade. Valeu!

As minhas amigas Gerusa, Daniela, Neyma, Beth e Lígia, obrigada por sempre me apoiarem e me ouvirem. Agradeço aos meus amigos: Carol, Cida, Miriam, Vera, Dona Helena, seu Celso, Dona Deolinda (obrigada pelas oraçōes), Camila, Fran, Karen e Priscila, que sempre torceram por mim. Obrigada!

A todos os professores que fizeram parte da minha vida acadêmica e sempre me incentivaram a prosseguir em meus estudos sem desistir de meus objetivos. Agradeço em especial aos professores Abramo Hefez e Marcelo Escudeiro Hernandes pela atenção que me dispensaram.

Enfim, agradeço a todos aqueles que de alguma forma colaboraram para a realização deste trabalho. 


\section{Resumo}

O objetivo deste trabalho é estudar as curvas algebróides planas. O Teorema de Newton-Puiseux permite obter uma parametrização especial destas curvas chamada de parametrização de Puiseux. Apresentamos o processo de desingularização que consiste em transformar uma curva singular numa curva regular através de sucessivos blowing-ups (transformações quadráticas especiais de $\mathbb{C}^{2}$ ). Também estudamos o índice de interseção entre duas curvas e o semigrupo associado a uma curva, assim como as relações entre esses conceitos. Finalmente mostramos que o número de Milnor de uma curva é igual ao condutor do semigrupo associado a ela e ambos são invariantes da curva. 


\section{Abstract}

Our main subject are the algebroid irreducible plane curves or plane branches. We show that every plane branch has a special kind of parametrization, the so called Puiseux parametrization, and that a singular plane branch can be turned into a regular one, through blowing-ups. We also study the intersection index of two plane branches and the semigroup of values associated to a plane branch. Finally we estabilish the equality between the semigroup's conductor and the Milnor number of the branch. 


\section{Sumário}

Introdução $\quad$ v

1 Séries de potências 1

1.1 Anéis de séries de potências . . . . . . . . . . . . . . . . . . . 1

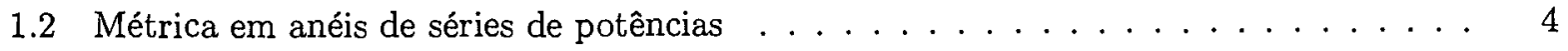

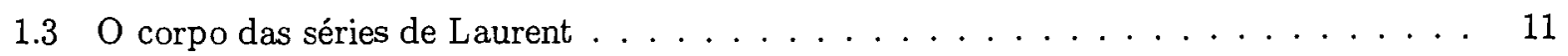

1.4 Lema de Hensel . . . . . . . . . . . . . . . . . . . . . . . . . . 12

2 O Teorema da Preparação 15

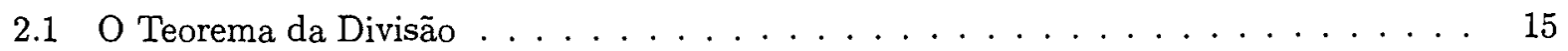

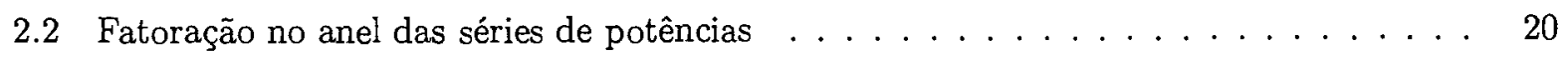

2.3 Teorema da base de Hilbert-Rückert . . . . . . . . . . . . . . . . 23

2.4 Eliminação . . . . . . . . . . . . . . . . . . . 24

3 Curvas Planas 31

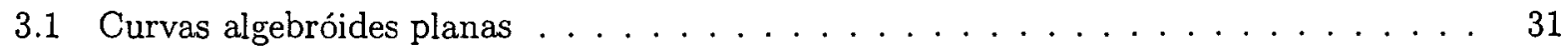

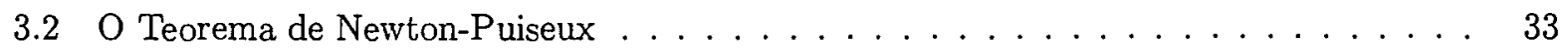

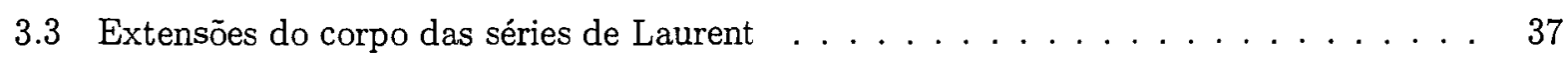

3.4 Parametrização e expoentes característicos . . . . . . . . . . . . . . . . . . . 42

3.5 Curvas analíticas planas . . . . . . . . . . . . . . . . . 46

4 Interseção de Curvas $\quad 53$

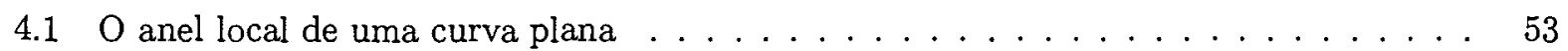

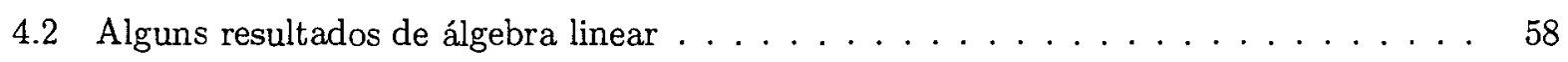

4.3 Índice de interseção . . . . . . . . . . . . . . . . . . . . . . . . . 61

5 Resolução de singularidades $\quad 67$

5.1 Transformações quadráticas em $\mathbb{C}^{2} \ldots \ldots \ldots \ldots \ldots \ldots \ldots \ldots$

5.2 Resolução de singularidades de curvas planas $\ldots \ldots \ldots \ldots \ldots \ldots$

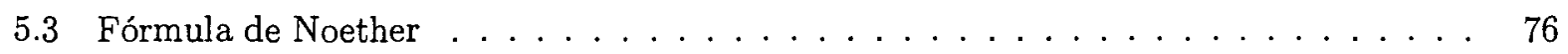

6 Semigrupos de Ramos Planos $\quad \mathbf{7 9}$

6.1 Semigrupos dos Naturais . . . . . . . . . . . . . . . . . . . . . 79

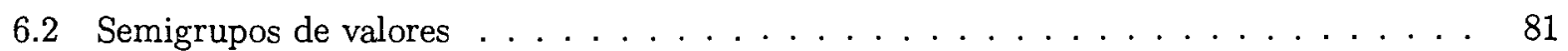

6.3 Semigrupos e equações cartesianas $\ldots \ldots \ldots \ldots \ldots \ldots \ldots$ 
6.4 Sequência de Apéry de um semigrupo de valores . . . . . . . . . . . . . . . . 91

6.5 Semigrupos e blowing-ups . . . . . . . . . . . . . . . . . 96

7 Semigrupos dos Naturais $\quad 99$

7.1 Semigrupos com condutores . . . . . . . . . . . . . . . . . . . . . . 99

7.2 Semigrupos fortemente crescentes . . . . . . . . . . . . . . . . . 104

7.3 Sequências de Apéry . . . . . . . . . . . . . . . . . . . 106

7.4 Aplicação ao semigrupos de valores . . . . . . . . . . . . . . . 109

$\begin{array}{ll}\text { Referências Bibliográficas } & 115\end{array}$ 


\section{Lista de Figuras}

5.1 Seqüência de blowing-ups (cúbica nodal) $\ldots \ldots \ldots \ldots \ldots \ldots$

5.2 Sequência de blowing-ups (cúspide) $\ldots \ldots \ldots \ldots \ldots \ldots \ldots \ldots$

5.3 Seqüência de blowing-ups (cúspide de ordem 2$) \ldots \ldots \ldots \ldots \ldots$ 


\section{Introdução}

O objetivo desta dissertação é estudar as singularidades de curvas irredutíveis planas do ponto de vista algébrico. O estudo local de uma curva algébrica $\mathcal{C}$ em $\mathbb{C}^{2}$ dada por

$$
\mathcal{C}=\left\{(x, y) \in \mathbb{C}^{2} ; f(x, y)=0\right\}
$$

onde $f(X, Y)$ é um polinômio em $\mathbb{C}[X, Y]$, na vizinhança de um ponto $P=(a, b) \in \mathcal{C}$, depende de $P$ ser ponto regular ou singular da curva.

Quando o ponto $P$ é regular, isto é, $f_{X}(P) \neq 0$ ou $f_{Y}(P) \neq 0$, o Teorema da Função Implícita nos diz que numa vizinhança do ponto $P$ podemos escrever $Y$ como uma série de potências em $X$, convergente numa vizinhança de $a$ em $\mathbb{C}$ (ou $X$ como uma série de potências em $Y$, convergente numa vizinhança de $b \mathrm{em} \mathbb{C}$ ). Assim, nesta vizinhança de $P$, a curva é o gráfico de uma função analítica em uma variável. Quando o ponto $P$ é singular, ou seja, quando $f(P)=f_{X}(P)=f_{Y}(P)=0$, Newton propôs uma solução para o problema explicitando $Y$ em função de $X$, usando séries de potências com expoentes fracionários. $O$ estudo de Newton era formal, sem a preocupação de verificar a convergência das séries em questão. Apesar disto, Newton forneceu um algoritmo para determinar tal desenvolvimento, chamado de polígono de Newton.

Em 1950, M.V. Puiseux estudou, do ponto de vista das funções de variável complexa, as soluções da equação $f(X, Y)=0$, onde $f$ é uma função polinomial em duas variáveis complexas, na vizinhança de um ponto. Puiseux justificou, do ponto de vista analítico, a construção de Newton das séries com expoentes fracionários.

Quando a curva é regular, segue do Teorema da Função Implícita, que ela é localmente isomorfa a $Y=0$. Quando o ponto é singular, a situação é diferente. $O$ anel $\mathbb{C}[X, Y]$ não é apropriado para estudar tais situações, pois o polinômio pode ser irredutível e a curva pode ser localmente redutível. No anel $\mathbb{C}[[X, Y]]$ das séries de potências formais é que vamos obter as informações algébricas a respeito da curva $\mathcal{C}$. Assim, a curva $\mathcal{C}$ é dada por $f=0$, onde $f$ é uma série de potências irredutível, e é chamada de curva algebróide irredutível plana. No entanto, mostramos que $f$ é equivalente a uma série mais simples, um polinômio de Weierstrass. Também obtemos uma parametrização especial para $f$ : a parametrização de Puiseux. Neste contexto, obtemos critérios algébricos para a equivalência de curvas e como determinar o índice de interseção entre duas curvas. Vamos estudar o processo de desingularização de uma curva que consiste em transformar uma curva singular em uma curva regular. Isso será feito através de blowing-ups, que são transformações quadráticas especiais em $\mathbb{C}^{2}$. Também estudamos as relações entre as curvas e seus blowing-ups, no que diz respeito ao índice de interseção e aos semigrupos de valores de curvas algebróides irredutíveis planas, um importante invariante de curvas. 
Estudamos as singularidades de curvas irredutíveis planas utilizando o método de Puiseux e temos como referência básica o artigo [1].

No capítulo 1 apresentamos o anel $\mathcal{R}=k\left[\left[X_{1}, \ldots, X_{n}\right]\right]$ das séries de potências formais, onde $k$ é um corpo, e estudamos algumas propriedades com respeito à topologia deste anel. Vimos que $\mathcal{R}$ é um espaço métrico completo e que todo homomorfismo de $k\left[\left[Y_{1}, \ldots, Y_{s}\right]\right]$ em $\mathcal{R}$ é dado por uma aplicação de substituição. Também apresentamos o corpo das séries de Laurent e o Lema de Hensel.

No capítulo 2 demonstramos o Teorema de Divisão, do qual segue o Teorema da Preparação de Weierstrass. Definimos séries de potências regulares em relação a uma indeterminada, pseudopolinômios e polinômios de Weierstrass. Mostramos que $\mathcal{R}$ é um domínio de fatoração única Noetheriano e definimos a resultante entre dois polinômios com coefientes em um domínio de fatoração única. Também mostramos algumas propriedades da resultante entre duas curvas e definimos o discriminante de um polinômio.

No capítulo 3 definimos curvas algebróides planas e aplicamos os resultados do capítulo 2 para mostrar que toda curva algebróide em $k[[X, Y]]$ é equivalente a um polinômio de Weierstrass, se $k$ é um corpo infinito. Também demonstramos o Teorema de Newton-Puiseux, que tem como consequência o fato de que toda curva algebróide irredutível possui uma parametrização de Puiseux. Terminamos o capítulo com uma abordagem especial para o caso $k=\mathbb{C}$, dando ênfase à questão da convergência das séries.

No capítulo 4 apresentamos ó anel local de uma curva algebróide plana e mostramos que o anel local de duas tais curvas são isomorfos se, e somente se, as curvas são equivalentes. Também definimos o índice de interseção entre duas curvas, obtemos suas propriedades, sua unicidade e mostramos como calcular o índice de interseção através dessas propriedades. Ainda, mostramos que o índice de interseção entre duas curvas é igual à multiplicidade da resultante dessas curvas.

No capítulo 5 utilizamos blowing-ups (transformações quadráticas especiais em $\mathbb{C}^{2}$ ) para construir o processo de desingularização de uma curva, que consiste em transformar uma curva singular em uma curva regular. Definimos a sequência de multiplicidades de uma curva através deste processo e mostramos o Teorema de Max Noether que relaciona o índice de interseção entre duas curvas planas irredutíveis e suas sequências de blowing-ups.

No capitulo 6 apresentamos o semigrupo de valores associado a uma curva algebróide irredutível plana, que é um importante invariante de tais curvas. Também relacionamos este semigrupo com a sequência de multiplicidades que obtemos quando estudamos o processo de desingularização. Além disso, utilizamos os inteiros característicos de uma curva para encontrar o semigrupo de uma curva dada pela equação cartesiana.

O Teorema de Milnor diz que o número de Milnor de uma curva irredutível plana é igual ao condutor do semigrupo de valores associado a ela. Este teorema, que é o objetivo central do capítulo 7, foi provado primeiramente por J.Milnor através de ferramentas topológicas e posteriormente por J.J.Risler com ferramentas algébricas. Assim, o número de Milnor de uma curva é um invariante da relação de equivalência entre curvas assim como o condutor. 


\section{Capítulo 1}

\section{Séries de potências}

\subsection{Anéis de séries de potências}

Seja $k$ um corpo e $X_{1}, \ldots, X_{r}$ indeterminadas sobre $k$. Denotaremos por $\mathcal{R}=k\left[\left[X_{1}, \ldots, X_{r}\right]\right]$ o conjunto das somas formais do tipo:

$$
f=\sum_{i=0}^{\infty} P_{i}=P_{0}+P_{1}+P_{2}+\ldots,
$$

onde cada $P_{i}$ é um polinômio homogêneo de grau $i$ nas indeterminadas $X_{1}, \ldots, X_{r}$ com coeficientes em $k$.

Sejam $f=P_{0}+P_{1}+\ldots$ e $g=Q_{0}+Q_{1}+\ldots$ elementos de $\mathcal{R}$, dizemos que $f=g$ se, e somente se, $P_{i}=Q_{i}, \forall i$. Definimos em $\mathcal{R}$ as seguintes operações:

$$
\begin{gathered}
f+g=\sum_{i=0}^{\infty} P_{i}+Q_{i} \\
f . g=\sum_{i=0}^{\infty} \sum_{j=0}^{i} P_{j} Q_{i-j}
\end{gathered}
$$

com estas operações $\mathcal{R}$ é um anel comutativo com unidade, chamado de anel das séries de potências formais em $r$ indeterminadas.

Os elementos de $\mathcal{R}$ podem ser representados na forma

$$
f=\sum_{i=0}^{\infty} \sum_{i_{1}+\ldots+i_{r}=i} a_{i_{1}, \ldots, i_{r}} X_{1}^{i_{1}} \ldots X_{r}^{i_{r}}
$$

$\operatorname{com} a_{i_{1}, \ldots, i_{r}} \in k$.

Proposição 1.1.1 O elemento $f=P_{0}+P_{1}+\ldots$ em $\mathcal{R}$ é invertível se, e somente se, $P_{0} \neq 0$.

\section{Demonstração:}

Suponhamos $f$ invertível e seja $f^{-1}=Q_{0}+Q_{1}+\ldots$ o inverso de $f$ em $\mathcal{R}$. Então

$$
\begin{gathered}
1=f \cdot f^{-1}=\left(P_{0}+P_{1}+\ldots\right)\left(Q_{0}+Q_{1}+\ldots\right) \\
P_{0} Q_{0}+\left(P_{1} Q_{0}+P_{0} Q_{1}\right)+\left(P_{2} Q_{0}+P_{1} Q_{1}+P_{0} Q_{2}\right)+\ldots
\end{gathered}
$$


Portanto, $P_{0} Q_{0}=1$, e como $P_{0}, Q_{0} \in k$, segue que $P_{0} \neq 0$.

Reciprocamente, suponhamos que $P_{0} \neq 0$. Se existisse o inverso $f^{-1}$ de $f, f^{-1}=Q_{0}+Q_{1}+\ldots$ deveria satisfazer

$$
1=P_{0} Q_{0}+\left(P_{1} Q_{0}+P_{0} Q_{1}\right)+\left(P_{0} Q_{2}+P_{1} Q_{1}+P_{2} Q_{0}\right)+\ldots
$$

e assim poderia ser determinado pelas relações:

$$
\begin{array}{ll}
P_{0} Q_{0}=1 & Q_{0}=P_{0}^{-1} \\
P_{1} Q_{0}+P_{0} Q_{1}=0 & Q_{1}=-P_{0}^{-1}\left(P_{1} Q_{0}\right) \\
P_{2} Q_{0}+P_{1} Q_{1}+P_{0} Q_{2}=0 & \Longrightarrow \\
\vdots & Q_{2}=-P_{0}^{-1}\left(P_{2} Q_{0}+P_{1} Q_{1}\right) \\
P_{n} Q_{0}+P_{n-1} Q_{1}+\ldots+P_{0} Q_{n}=0 & \vdots \\
& Q_{n}=-P_{0}^{-1}\left(P_{n} Q_{0}+P_{n-1} Q_{1}+\ldots+P_{1} Q_{n-1}\right)
\end{array}
$$

Como $P_{0} \neq 0$, temos que $f^{-1}$ está bem definida em $\mathcal{R}$.

Definição 1.1.2 Seja $f \in \mathcal{R} \backslash\{0\}$. Suponhamos que $f=P_{n}+P_{n+1}+\ldots$, onde cada $P_{j}$ é um polinômio homogêneo de grau $j$ e $P_{n} \neq 0$. O polinômio homogêneo $P_{n}$ é chamado de forma inicial de $f$ e o inteiro $n$ é chamado de multiplicidade de $f$ e será denotado por mult $(f)$. Se $f=0$ definimos mult $(f)=\infty$.

Segue da Proposição 1.1.1 que $f$ é invertível se, e somente se, mult $(f)=0$. A multiplicidade satisfaz as seguintes propriedades:

Proposição 1.1.3 Sejam $f, g \in \mathcal{R}$. Temos que

(i) $\operatorname{mult}(f \cdot g)=\operatorname{mult}(f)+$ mult $(g)$.

(ii) $\operatorname{mult}(f \pm g) \geq \min \{\operatorname{mult}(f)$, mult $(g)\}$, com igualdade ocorrendo sempre que mult $(f) \neq$ mult $(g)$.

\section{Demonstração:}

(i) Sejam $f, g \in \mathcal{R}, f=P_{n}+P_{n+1}+\ldots$ e $g=Q_{m}+Q_{m+1}+\ldots$, com mult $(f)=n$ e mult $(g)=m$. Temos que

$$
f \cdot g=\left(P_{n} Q_{m}\right)+\left(P_{n+1} Q_{m}+P_{n} Q_{m+1}\right)+\ldots
$$

onde $P_{n} Q_{m}$ é um polinômio homogêneo não nulo de grau $n+m$, pois o anel dos polinômios é um domínio. Logo, mult $(f \cdot g)=n+m=\operatorname{mult}(f)+\operatorname{mult}(g)$.

Se $f=0$ ou $g=0$, temos que $f \cdot g=0$. Assim, mult $(f \cdot g)=\infty=\operatorname{mult}(f)+$ mult $(g)$.

(ii) Suponhamos $n<m$ e sejam $f=P_{n}+P_{n+1}+\ldots$ e $g=Q_{m}+Q_{m+1}+\ldots$, com mult $(f)=n$ e mult $(g)=m$. Temos que

$$
f \pm g=P_{n}+P_{n+1}+\ldots+P_{m-1}+\left(P_{m} \pm Q_{m}\right)+\left(P_{m+1} \pm Q_{m+1}\right)+\ldots
$$

Portanto mult $(f \pm g)=n=\operatorname{mult}(f)=\min \{\operatorname{mult}(f)$, mult $(g)\}$. 
Se $f=P_{n}+P_{n+1}+\ldots$ e $g=Q_{n}+Q_{n+1}+\ldots$, com mult $(f)=\operatorname{mult}(. g)=n$, então

$$
f \pm g=\left(P_{n} \pm Q_{n}\right)+\left(P_{n+1} \pm Q_{n+1}\right)+\ldots
$$

Notemos que $P_{n} \pm Q_{n}$ pode ser um polinômio homogêneo de grau $n$ ou pode ser o polinômio nulo. Portanto, mult $(f \pm g) \geq n=\operatorname{mult}(f)=\operatorname{mult}(g)$.

Assim, $\forall f, g \in \mathcal{R}$, temos que mult $(f \pm g) \geq \min \{$ mult $(f)$, mult $(g)\}$.

Proposição 1.1.4 $O$ anel $\mathcal{R}$ é um domínio.

Demonstração: Sabendo que $\mathcal{R}$ é um anel comutativo com unidade, basta mostrar que $\mathcal{R}$ não possui divisores de zero. Sejam $f, g \in \mathcal{R} \backslash\{0\}$. Da Proposição 1.1.3(1), segue que

$$
\text { mult }(f \cdot g)=\operatorname{mult}(f)+\operatorname{mult}(g)<\infty
$$

pois $f, g \neq 0$. Como mult $(0)=\infty$, segue que $f \cdot g \neq 0$.

Denotaremos por $\mathcal{M}_{\mathcal{R}}=\left\langle X_{1}, \ldots, X_{r}\right\rangle$ o ideal de $\mathcal{R}$ gerado por $X_{1}, \ldots, X_{r}$. Vamos denotar por $\mathcal{M}_{\mathcal{R}}^{i}$ a $i$-ésima potência do ideal $\mathcal{M}_{\mathcal{R}}$ e por $\mathcal{M}_{\mathcal{R}}^{0}$ o domínio $\mathcal{R}$.

Proposição 1.1.5 $O$ ideal $\mathcal{M}_{\mathcal{R}}$ é o único ideal maximal de $\mathcal{R}$ e é tal que

$$
\bigcap_{i \in \mathbb{N}} \mathcal{M}_{\mathcal{R}}^{i}=\{0\}
$$

\section{Demonstração:}

Mostremos que se $f \in \mathcal{R} \backslash \mathcal{M}_{\mathcal{R}}$ então $f$ é invertível. Como

$$
\mathcal{M}_{\mathcal{R}}=\left\{f_{1} X_{1}+\ldots+f_{r} X_{r}: f_{1}, \ldots, f_{r} \in R\right\}
$$

temos que todos os elementos de $\mathcal{M}_{\mathcal{R}}$ tem multiplicidade $\geq 1$. Logo, $\mathcal{R} \backslash \mathcal{M}_{\mathcal{R}}=\{f \in \mathcal{R}:$ mult $f \neq 0\}$, que pela Proposição 1.1.1 são os elementos invertiveis de $\mathcal{R}$. Assim, $\mathcal{R}$ é um anel local e $\mathcal{M}_{\mathcal{R}}$ é seu único ideal maximal.

Mostremos que $\bigcap_{i \in \mathbb{N}} \mathcal{M}_{\mathcal{R}}^{i}=\{0\}$. Temos que

$$
\mathcal{M}_{\mathcal{R}}^{i}=\left\langle X_{i_{1}} \ldots X_{i_{j}}\right\rangle
$$

$\operatorname{com} i_{1}, \ldots, i_{j}=1, \ldots, r, i_{1}+\ldots+i_{j}=i$. Como $0 \in \mathcal{M}_{\mathcal{R}}^{i}, \forall i$, segue que $\{0\} \subset \bigcap_{i \in \mathbb{N}} \mathcal{M}_{\mathcal{R}}^{i}$.

Se $f \in \mathcal{M}_{\mathcal{R}}^{i}, \forall i \in \mathbb{N}$, entāo $f=P_{i}+P_{i+1}+\ldots$ Mas $f \in \mathcal{M}_{\mathcal{R}}^{i+1} ; \operatorname{logo} P_{i}=0, \forall i$. Segue que $f=0$, e portanto

$$
\bigcap_{i \in \mathbb{N}} \mathcal{M}_{\mathcal{R}}^{i}=\{0\} .
$$




\subsection{Métrica em anéis de séries de potências}

Para dar um significado à soma infinita de séries de potências, será conveniente definir uma métrica nestes conjuntos infinitos de séries. Isto é o que faremos a seguir.

Seja $\mathcal{R}=k\left[\left[X_{1}, \ldots, X_{r}\right]\right]$ e $\mathcal{M}_{\mathcal{R}}$ seu ideal maximal. Seja $\rho>1$ um número real e consideremos a seguinte aplicação

$$
\begin{aligned}
d: \mathcal{R} \times \mathcal{R} & \longrightarrow \mathbb{R} \\
(f, g) & \longmapsto \begin{cases}\rho^{- \text {mult }(f-g)} & \text { se } f \neq g \\
0 & \text { se } f=g\end{cases}
\end{aligned}
$$

Proposição 1.2.1 $O \operatorname{par}(\mathcal{R}, d)$ é um espaço métrico completo.

\section{Demonstração:}

Mostremos primeiramente que $d$ é uma métrica em $\mathcal{R}$.

Como $\rho>1$ e mult $(f) \geq 0, \forall f \in \mathcal{R}$, temos que

$$
d(f, g)=\rho^{- \text {mult }(f-g)}=\frac{1}{\rho^{\text {mult }(f-g)}} \geq 0
$$

e $d(f, g)=0$ se, e somente se, $f=g$.

Sabemos que mult $(f-g)=$ mult $(g-f)$, e portanto $d(f, g)=d(g, f)$.

A desigualdade triangular segue da seguinte desigualdade

$$
\text { mult }(f-g)=\operatorname{mult}((f-h)-(g-h)) \geq \min \{\operatorname{mult}(f-h), \text { mult }(g-h)\}:=m
$$

e do fato de $\rho>1$, pois

$$
d(f, g)=\rho^{- \text {mult }(f-g)} \leq \rho^{-m} \leq \rho^{- \text {mult }(f-h)}+\rho^{- \text {mult }(g-h)}=d(f, h)+d(h, g) .
$$

Para mostrar que $(\mathcal{R}, d)$ é um espaço métrico completo, seja $\left(f_{n}\right)_{n \in \mathbb{N}}$ uma sequência de Cauchy em $\mathcal{R}$, ou seja, $\forall n \in \mathbb{N}$, existe um inteiro $\nu(n)$ tal que $d\left(f_{l}, f_{m}\right)=\rho^{-\operatorname{mult}\left(f_{l}-f_{m}\right)}<\rho^{-n}, \forall l, m \geq \nu(n)$. Assim, mult $\left(f_{l}-f_{m}\right)>n$ e portanto $f_{l}-f_{m} \in \mathcal{M}_{\mathcal{R}}^{n}, \forall l, m \geq \nu(n)$.

Podemos escolher $\nu(n)$ de forma que $(\nu(n))$ seja uma sequência crescente de números inteiros e assim teremos $f_{\nu(n)}-f_{\nu(n+1)} \in \mathcal{M}_{\mathcal{R}}^{n}, \forall n \in \mathbb{N}$.

Para cada $i \in \mathbb{N}$, escrevemos $f_{\nu(i)}=P_{i, 0}+P_{i, 1}+\ldots$, onde cada $P_{i, j}$ é um polinômio homogêneo de grau $j$, e definimos $f=P_{1,0}+P_{2,1}+P_{3,2}+\ldots+P_{i+1, i}+\ldots$ Temos que

$$
\begin{aligned}
& f-f_{\nu(1)}=\underline{P_{1,0}}+P_{2,1}+P_{3,2}+\ldots-\underline{P_{1,0}}-P_{1,1}-P_{1,2}-\ldots \in \mathcal{M}_{\mathcal{R}} \\
& f-f_{\nu(2)}=P_{1,0}+\underline{P_{2,1}}+P_{3,2}+\ldots-P_{2,0}-\underline{P_{2,1}}-P_{2,2}-\ldots \in \mathcal{M}_{\mathcal{R}}^{2}
\end{aligned}
$$

pois como $f_{\nu(1)}-f_{\nu(2)} \in \mathcal{M}_{\mathcal{R}}$, temos que $P_{1,0}-P_{2,0}=0$.

Temos que

$$
f-f_{\nu(i)}=P_{1,0}+P_{2,1}+\ldots+P_{i-1, i-2}+\underline{P_{i, i-1}}+\ldots-P_{i, 0}-P_{i, 1}-\ldots-P_{i, i-2}-\underline{P_{i, i-1}}-P_{i, i}+\ldots
$$

Mas, $f_{\nu(j)}-f_{\nu(j+1)} \in \mathcal{M}_{\mathcal{R}}^{j}, j=1, \ldots, i-1$, donde

$$
P_{1,0}-P_{i, 0}+P_{2,1}-P_{i, 1}+\ldots+P_{i-1, i-2}-P_{i, i-2}=0 .
$$


Assim, $f-f_{\nu(i)} \in \mathcal{M}_{\mathcal{R}}^{i}, \forall i \in \mathbb{N}$, o que implica que

$$
\lim _{n \rightarrow \infty} f_{\nu(n)}=f
$$

Isto mostra que a sequência de Cauchy $\left(f_{n}\right)$ tem uma subsequência $\left(f_{\nu(n)}\right)$ que converge para $f \in \mathcal{R}$; portanto a sequência $\left(f_{n}\right)$ converge para $f$. Logo, o par $(\mathcal{R}, d)$ é um espaço métrico completo.

A definição a seguir é um critério para dizer quando uma família infinita de séries de potências é somável.

Definição 1.2.2 Seja $\mathcal{F}=\left\{f_{\lambda} ; \lambda \in \Lambda\right\}$ uma familia de séries de potências em $\mathcal{R}$. Dizemos que a familia $\mathcal{F}$ é somável se $\forall n \in \mathbb{N}$,

$$
\#\left\{\lambda \in \Lambda ; \operatorname{mult}\left(f_{\lambda}\right) \leq n\right\}<\infty .
$$

Proposição 1.2.3 Sejam $\left\{f_{\lambda} ; \lambda \in \Lambda\right\}$ e $\left\{g_{\lambda} ; \lambda \dot{\in} \Lambda\right\}$ duas familias somáveis em $\mathcal{R}$ e sejam $A, B \in \mathcal{R}$. Então

(i) A soma $\sum_{\lambda \in \Lambda} f_{\lambda}$ é bem definida em $\mathcal{R}$.

(ii) A familia $\left\{A f_{\lambda}+B g_{\lambda} ; \lambda \in \Lambda\right\}$ é somável $e$

$$
\sum_{\lambda \in \Lambda}\left(A f_{\lambda}+B g_{\lambda}\right)=A \sum_{\lambda \in \Lambda} f_{\lambda}+B \sum_{\lambda \in \Lambda} g_{\lambda} .
$$

(iii) A familia $\left\{f_{\lambda} g_{\mu} ;(\lambda, \mu) \in \Lambda \times \Lambda\right\}$ é somável e

$$
\left(\sum_{\lambda \in \Lambda} f_{\lambda}\right)\left(\sum_{\mu \in \Lambda} g_{\mu}\right)=\sum_{(\lambda, \mu) \in \Lambda \times \Lambda} f_{\lambda} g_{\mu} .
$$

\section{Demonstração:}

(i) Como a familia $\left\{f_{\lambda}\right\}$ é somável, o conjunto

$$
\Lambda_{n}=\left\{\lambda \in \Lambda ; \operatorname{mult}\left(f_{\lambda}^{\prime}\right) \leq n\right\}
$$

é finito. Seja

$$
h_{n}=\sum_{\lambda \in \Lambda_{n}} f_{\lambda}
$$

Se $m>n$, então

$$
\Lambda_{m}=\Lambda_{n} \cup\left\{\lambda \in \Lambda ; n<\operatorname{mult}\left(f_{\lambda}\right) \leq m\right\} .
$$

Logo, $\forall m \geq n$, temos que mult $\left(h_{m}-h_{n}\right) \geq \min \left\{\operatorname{mult}\left(h_{m}\right)\right.$, mult $\left.\left(h_{n}\right)\right\}>n$. Assim,

$$
d\left(h_{m}, h_{n}\right)=\rho^{- \text {mult }\left(h_{m}-h_{n}\right)}<\rho^{-n}, \forall m \geq n .
$$

Logo $\left(h_{n}\right)$ é uma sequência de Cauchy, e portanto converge para um elemento de $\mathcal{R}$, o qual denotaremos por $\sum_{\lambda \in \Lambda} f_{\lambda}$. 
(ii) Dado $n \in \mathbb{N}$, definimos

$$
\Lambda_{n}^{\prime \prime}=\left\{\lambda \in \Lambda ; \text { mult }\left(A f_{\lambda}+B g_{\lambda}\right) \leq n\right\}
$$

Se $A=B=0$, então $A f_{\lambda}+B g_{\lambda}=0$. Como mult $(0)=\infty$, segue que $\Lambda_{n}^{\prime \prime}=\emptyset, \forall n$, e, portanto, é finito. Temos que

$$
n \geq \operatorname{mult}\left(A f_{\lambda}+B g_{\lambda}\right) \geq \min \left\{\operatorname{mult}\left(A f_{\lambda}\right), \operatorname{mult}\left(B g_{\lambda}\right)\right\} .
$$

Logo

$$
\min \left\{\operatorname{mult}(A)+\operatorname{mult}\left(f_{\lambda}\right), \operatorname{mult}(B)+\operatorname{mult}\left(g_{\lambda}\right)\right\} \leq n
$$

e existe apenas um número finito de índices que satisfaz a desigualdade acima. Portanto, a família $\left\{A f_{\lambda}+B g_{\lambda}\right\}$ é somável.

Sejam $\Lambda_{n}=\left\{\lambda \in \Lambda ;\right.$ mult $\left.\left(f_{\lambda}\right) \leq n\right\}$ e $\Lambda_{n}^{\prime}=\left\{\lambda \in \Lambda ;\right.$ mult $\left.\left(g_{\lambda}\right) \leq n\right\}$. Definimos

$$
\phi_{n}=\sum_{\lambda \in \Lambda_{n}^{\prime \prime}}\left(A f_{\lambda}+B g_{\lambda}\right)
$$

e

$$
\psi_{n}=A \sum_{\lambda \in A_{n}} f_{\lambda}+B \sum_{\lambda \in \Lambda_{n}^{\prime}} g_{\lambda}
$$

Temos que $\dot{\psi}_{n}-\phi_{n} \in \mathcal{M}_{\mathcal{R}}^{n+1}$, pois mult $\left(\iota_{n}-\phi_{n}\right)>n, \forall n \in \mathbb{N}$.

Logo

$$
\lim _{n \rightarrow \infty}\left(\dot{u}_{n}-\phi_{n}\right)=0
$$

Portanto

$$
\lim _{n \rightarrow \infty} \psi_{n}-\lim _{n \rightarrow \infty} \dot{\phi}_{n}=A \sum_{\lambda \in \Lambda} f_{\lambda}+B \sum_{\lambda \in \Lambda} g_{\lambda}-\sum_{\lambda \in \Lambda}\left(A f_{\lambda}+B g_{\lambda}\right)=0
$$

O que implica

$$
A \sum_{\lambda \in \Lambda} f_{\lambda}+B \sum_{\lambda \in \Lambda} g_{\lambda}=\sum_{\lambda \in \Lambda}\left(A f_{\lambda} \div B g_{\lambda}\right)
$$

(iii) Definimos

$$
\Lambda_{n}^{\prime \prime \prime}=\left\{(\lambda, \mu) \in \Lambda \times \Lambda ; \operatorname{mult}\left(f_{\lambda} g_{\mu}\right) \leq n\right\}
$$

Mas

$$
\text { mult }\left(f_{\lambda} g_{\mu}\right)=\operatorname{mult}\left(f_{\lambda}\right)+\operatorname{mult}\left(g_{\mu}\right)
$$

e existe um número finito de índices $(\lambda, \mu) \in \Lambda \times \Lambda$ tais que mult $\left(f_{\lambda} g_{\mu}\right) \leq n$, pois as familias $\left\{f_{\lambda}\right\}$ e $\left\{g_{\mu}\right\}$ são somáveis. Logo a família $\left\{f_{\lambda} g_{\mu} ;(\lambda, \mu) \in \Lambda \times \Lambda\right\}$ é somável.

Definimos

$$
\begin{gathered}
\phi_{n}=\sum_{(\lambda, \mu) \in \Lambda_{n}^{\prime \prime \prime}} f_{\lambda} g_{\mu} \\
\psi_{n}=\left(\sum_{\lambda \in \Lambda_{n}} f_{\lambda}\right)\left(\sum_{\mu \in \Lambda_{n}^{\prime}} g_{\mu}\right)
\end{gathered}
$$

Temos que $\psi_{n}-\phi_{n} \in \mathcal{M}_{\mathcal{R}}^{n+1}$, pois mult $\left(\imath_{n}-\phi_{n}\right)>n, \forall n \in \mathbb{N}$. 
Logo

$$
\lim _{n \rightarrow \infty}\left(\psi_{n}-\phi_{n}\right)=0
$$

Portanto

$$
0=\lim _{n \rightarrow \infty} \psi_{n}-\lim _{n \rightarrow \infty} \phi_{n}=\left(\sum_{\lambda \in \Lambda} f_{\lambda}\right)\left(\sum_{\mu \in \Lambda} g_{\mu}\right)-\sum_{(\lambda, \mu) \in \Lambda \times \Lambda} f_{\lambda} g_{\mu}
$$

O que implica o resultado.

Seja $\left\{P_{i} ; i \in \mathbb{N}\right\}$ uma família de polinômios homogêneos, com $P_{i} \in k\left[Y_{1}, \ldots, Y_{s}\right]$ de grau $i$ e sejam $g_{1}, \ldots, g_{s} \in \mathcal{M}_{\mathcal{R}}$

Consideremos a família $\mathcal{F}=\left\{P_{i}\left(g_{1}, \ldots, g_{s}\right) ; i \in \mathbb{N}\right\}$ e os conjuntos

$$
\Lambda_{i}=\left\{n \in \mathbb{N} ; \text { mult }\left(P_{n}\left(g_{1}, \ldots, g_{s}\right)\right) \leq i\right\}
$$

Se mult $\left(P_{n}\left(g_{1}, \ldots, g_{s}\right)\right) \leq i$ então $n \leq i$, pois o fato de que $g_{1}, \ldots, g_{s} \in \mathcal{M}_{\mathcal{R}}$ implica que mult $\left(P_{j}\left(g_{1}, \ldots, g_{s}\right)\right) \geq j, \forall j \in \mathbb{N}$. Assim, existe um número finito de números naturais $n$ para os quais mult $\left(P_{n}\left(g_{1}, \ldots, g_{s}\right)\right) \leq i$. Portanto, $\mathcal{F}$ é somável.

Denotando por $f=\sum_{i \in \mathbb{N}} P_{i}\left(Y_{1}, \ldots, Y_{s}\right) \in k\left[\left[Y_{1}, \ldots, Y_{s}\right]\right]$, então denotaremos a soma da família $\mathcal{F}$ por $f\left(g_{1}, \ldots, g_{s}\right)$ e será chamada de substituição de $Y_{1}, \ldots, Y_{s}$ por $g_{1}, \ldots, g_{s}$ em $f$.

Corolário 1.2.4 Dados $g_{1}, \ldots, g_{s} \in \mathcal{M}_{\mathcal{R}}, f, h \in k\left[\left[Y_{1}, \ldots, Y_{s}\right]\right]$ e $a \in k$, então

(i) $(f+a h)\left(g_{1}, \ldots, g_{s}\right)=f\left(g_{1}, \ldots, g_{s}\right)+a h\left(g_{1}, \ldots, g_{s}\right)$.

(ii) $(f \cdot h)\left(g_{1}, \ldots, g_{s}\right)=f\left(g_{1}, \ldots, g_{s}\right) \cdot h\left(g_{1}, \ldots, g_{s}\right)$.

(iii) $A$ aplicação de substituição.

$$
\begin{array}{cl}
\mathcal{S}_{g_{1}, \ldots, g_{s}}: k\left[\left[Y_{1}, \ldots, Y_{s}\right]\right] & \longrightarrow \mathcal{R}=k\left[\left[X_{1}, \ldots, X_{r}\right]\right] \\
-f & \longmapsto \mathcal{S}_{g_{1}, \ldots, g_{s}}(f)=f\left(g_{1}, \ldots, g_{s}\right)
\end{array}
$$

é um homomorfismo de k-álgebras.

\section{Demonstração:}

Sejam $f=\sum_{i \in \mathbb{N}} P_{i}\left(Y_{1}, \ldots, Y_{s}\right)$ e $h=\sum_{i \in \mathbb{N}} Q_{i}\left(Y_{1}, \ldots, Y_{s}\right)$, onde $P_{i}, Q_{i}$ são polinộmios homogêneos de grau i. Temos que $\mathcal{F}=\left\{P_{i}\left(g_{1}, \ldots, g_{s}\right) ; i \in \mathbb{N}\right\}$ e $\mathcal{H}=\left\{Q_{i}\left(g_{1}, \ldots, g_{s}\right) ; i \in \mathbb{N}\right\}$ são famílias somáveis, assim pela Proposição 1.2.3(ii) e (iii) seguem as propriedades $(i)$ e (ii) deste corolário. A propriedade (iii) segue das propriedades $(i)$ e $(i i)$.

Reciprocamente, todo homomorfismo de $k$-álgebras de $\mathcal{S}=k\left[\left[Y_{1}, \ldots, Y_{s}\right]\right]$ em $\mathcal{R}$ é uma aplicação de substituição. Denotando por $\mathcal{M}_{\mathcal{R}}$ e $\mathcal{M}_{\mathcal{S}}$ os respectivos ideais maximais de $\mathcal{R}$ e $\mathcal{S}$ tal resultado segue da seguinte proposição. 
Proposição 1.2.5 Seja $T: \mathcal{S} \longrightarrow \mathcal{R}$ um homomorfismo de k-álgebras. Então

(i) $T\left(\mathcal{M}_{\mathcal{S}}\right) \subset \mathcal{M}_{\mathcal{R}}$.

(ii) $T$ é contínua.

(iii) Existem $g_{1}, \ldots, g_{s} \in \mathcal{M}_{\mathcal{R}}$ tais que $T=\mathcal{S}_{g_{1}, \ldots, g_{s}}$.

\section{Demonstração:}

(i) Seja $f \in \mathcal{M}_{\mathcal{S}}$ e suponhamos que $T(f) \notin \mathcal{M}_{\mathcal{R}}$. Então podemos escrever $T(f)=c+g$, onde $c \in k \backslash\{0\}$ e $g \in \mathcal{M}_{\mathcal{R}}$. Como $T$ é um homomorfismo de $k$-álgebras, segue que

$$
T(f-c)=T(f)-c T(1)=T(f)-c=c+g-c=g .
$$

Mas $f-c$ é invertível em $\mathcal{S}$ enquanto que $g \in \mathcal{M}_{\mathcal{R}}$ não é invertível em $\mathcal{R}$, o que é uma contradição, pois $T$ é homomorfismo e portanto leva unidades de $\mathcal{S}$ em unidades de $\mathcal{R}$.

(ii) Segue de (1) que $T\left(\mathcal{M}_{\mathcal{S}}^{n}\right) \subset \mathcal{M}_{\mathcal{R}}^{n}$. Assim, mult $(f-g) \leq \operatorname{mult}(T(f)-T(g)), \forall f, g \in \mathcal{S}$, o que implica

$$
\rho^{-\operatorname{mult}(T(f)-T(g))} \leq \rho^{-\operatorname{mult}(f-g)} .
$$

Dado $f \in \mathcal{S}$ e $\epsilon>0$, tome $\delta \leq \epsilon$. Se $g \in \mathcal{S}$ é tal que $d(f, g)<\delta$. então $d(T(f), T(g)) \leq d(f, g)<$ $\delta \leq \epsilon$. Portanto $T$ é contínua.

(iii) Seja $g_{j}=T\left(Y_{j}\right), \forall j=1, \ldots, s$. De (i) temos que $g_{j} \in \mathcal{M}_{\mathcal{R}}, \forall j=1, \ldots, s$.

Seja $f=\sum_{i \in \mathbb{N}} P_{i} \in \mathcal{S}$. Como $T$ é homomorfismo, temos que

$$
T\left(\sum_{i=1}^{n} P_{i}\right)=\sum_{i=1}^{n} T\left(P_{i}\right)=\sum_{i=1}^{n} P_{i}\left(g_{1}, \ldots, g_{s}\right)
$$

Mas $\lim _{n \rightarrow \infty} \sum_{i=1}^{n} P_{i}=f$ e $\lim _{n \rightarrow \infty} \sum_{i=1}^{n} P_{i}\left(g_{1}, \ldots, g_{s}\right)=f\left(g_{1}, \ldots, g_{s}\right)$. Como $T$ é contínua, segue que

$$
T(f)=f\left(g_{1}, \ldots, g_{s}\right)=\mathcal{S}_{g_{1}, \ldots, g_{s}}(f) .
$$

Logo, $T=\mathcal{S}_{g_{1}, \ldots, g_{s}}$.

Então, dado um homomorfismo $\mathcal{T}: \mathcal{S} \longrightarrow \mathcal{R}$, existem $g_{1}, \ldots, g_{s} \in \mathcal{M}_{\mathcal{R}}$ tais que $T=\mathcal{S}_{g_{1}, \ldots, g_{s}}$. Vejamos agora quais as condições para que $T$ seja um $k$-isomornismo.

Se $f \in \mathcal{S}$ e $g_{1}, \ldots, g_{s} \in \mathcal{M}_{\mathcal{R}}$ então

$$
\operatorname{mult}\left(\mathcal{S}_{g_{1}, \ldots, g_{s}}(f)\right)=\operatorname{mult}\left(f\left(g_{1}, \ldots, g_{s}\right)\right) \geq \operatorname{mult}(f) \cdot \min _{i}\left\{\operatorname{mult}\left(g_{i}\right)\right\} \cdot \operatorname{mult}(f) .
$$

Se $T$ é um $k$-isomorfismo então

$$
\text { mult }(f) \leq \operatorname{mult}(T(f)) \leq \operatorname{mult}\left(T^{-1}(T(f))\right)=\operatorname{mult}(f) .
$$


Portanto, mult $(T(f))=$ mult $(f)$. Logo, todo $k$-isomorfismo de $\mathcal{S}$ em $\mathcal{R}$ deve preservar multiplicidades.

Como $T\left(Y_{i}\right)=g_{i}, \forall i=1, \ldots, s$, segue que

$$
\operatorname{mult}\left(g_{i}\right)=\operatorname{mult}\left(Y_{i}\right)=1, \forall i=1, \ldots, s .
$$

Sejam $L_{1}, \ldots, L_{s}$ as respectivas formas iniciais de $g_{1}, \ldots, g_{s}$ que são polinômios homogêneos de grau 1 em $X_{1}, \ldots, X_{r}$. Suponhamos que exista uma relação de dependência linear não trivial em $k$,

$$
a_{1} L_{1}+\ldots+a_{s} L_{s}=0 .
$$

Assim, tomando $f=a_{1} Y_{1}+\ldots+a_{s} Y_{s}$, teríamos

$$
\operatorname{mult}\left(\mathcal{S}_{g_{1}, \ldots, g_{s}}(f)\right)>1=\operatorname{mult}(f) \text {, }
$$

o que implicaria que $S_{g_{1}, \ldots, g_{s}}$ não é um $k$-isomorfismo."

Conseqüentemente uma condição necessária para que $\mathcal{S}_{g_{1}, \ldots, g_{s}}$ seja um $k$-isomorfismo de $\mathcal{S}$ em $\mathcal{R}$ é que as formas iniciais de $g_{1}, \ldots, g_{s}$ sejam linearmente independentes sobre $k$. Isto mostra, em particular, que $s \leq r$, e usando o mesmo argumento para $T^{-1}$ temos que $r \leq s$. Obtemos, assim que $r=s$.

Para mostrar que as condições acima são também suficientes vamos precisar do seguinte lema.

Lema 1.2.6 Um subanel $A$ de $\mathcal{R}$ é denso em $\mathcal{R}$ se, e somente se, para todo polinômio homogêneo $P \in k\left[X_{1}, \ldots, X_{r}\right]$ existe um elemento em $A$ cuja forma inicial é $P$.

\section{Demonstraçāo:}

Suponhamos que $A$ é denso em $\mathcal{R}$ e seja $P$ um polinômio homogêneo de grau $d$ em $k\left[X_{1}, \ldots, X_{r}\right]$. Seja $n \in \mathbb{N}, n>d$. Como $A$ é denso em $\mathcal{R}$ o polinômio $P$ é o limite de uma sequência de elementos de $A$ e portanto existe $f \in A$ tal que mult $\left(f^{\prime}-P\right) \geq n$. Como $n>d$, segue que $P$ é a forma inicial de $f$.

Reciprocamente, suponhamos que para todo polinômio homogêneo $P \in k\left[X_{1}, \ldots, X_{r}\right]$ existe um elemento em $A$ cuja forma inicial é $P$. Seja $f \in \mathcal{R}$. Vamos construir uma sequência infinita $\left(f_{i}\right)$ de elementos de $A$ tal que $\lim f_{i}=f$. Procederemos por indução. Seja $f_{0}=0$. Suponhamos que foram construídos elementos $f_{0}, f_{1}, \ldots, f_{n-1}$ de $A$ tais que

$$
\operatorname{mult}\left(f-f_{i}\right) \geq i, i=0, \ldots, n-1 .
$$

Se mult $\left(f-f_{n-1}\right) \geq n$, colocamos $f_{n}=f_{n-1}$.

Suponhamos mult $\left(f-f_{n-1}\right)=n-1$. Se $G_{n-1}$ é a forma inicial de $f-f_{n-1}$, então existe um elemento $h_{n-1}$ de $A$ cuja forma inicial é $G_{n-1}$. Definindo $f_{n}=f_{n-1}+h_{n-1}$, temos que $f_{n} \in A$ e mult $\left(f-f_{n}\right)=$ mult $\left(f-f_{n-1}-h_{n-1}\right) \geq n$, pois a forma inicial de $f-f_{n-1}$ é igual à forma inicial de $h_{n-1}$. Dessa forma construímos uma sequência de elementos de $A$ tais que $\lim f_{i}=f$. Logo $A$ é denso em $\mathcal{R}$.

Em particular, temos que $k\left[X_{1}, \ldots, X_{r}\right]$ é denso em $k\left[\left[X_{1}, \ldots, X_{r}\right]\right]$. 
Se $L_{1}, \ldots, L_{r}$ são formas lineares em $\mathcal{R}$ linearmente independentes sobre $k$, então $T=\mathcal{S}_{L_{1}, \ldots, L_{r}}$ é um $k$-isomorfismo de $\mathcal{S}$ em $\mathcal{R}$ quando $r=s$. De fato, se

$$
L_{i}=a_{i, 1} X_{1}+\ldots+a_{i, r} X_{r}, i=1, \ldots, r
$$

e se $A=\left(a_{i, j}\right)$ então $T^{-1}=\mathcal{S}_{M_{1}, \ldots, M_{r}}$, onde

$$
M_{i}=b_{i, 1} X_{1}+\ldots+b_{i, r} X_{r}, i=1, \ldots, r
$$

e $\left(b_{i, j}\right)$ é a matriz inversa de $A$.

Proposição 1.2.7 Suponhamos $r=s$ e sejam $g_{1}, \ldots, g_{r} \in \mathcal{R}$ com formas iniciais $L_{1}, \ldots, L_{r}$ lineares e linearmente independentes sobre $k$. Então $\mathcal{S}_{g_{1}, \ldots, g_{r}}$ é um $k$-isomorfismo de $\mathcal{S}$ em $\mathcal{R}$.

\section{Demonstração:}

Mostremos que $S_{g_{1}, \ldots, g_{r}}$ é injetora. Observemos que se $0 \neq f=P_{n}+P_{n+1}+\ldots$ onde cada $P_{i}$ é polinômio homogêneo de grau $i$ e $P_{n} \neq 0$, então o termo inicial de $\mathcal{S}_{g_{1}, \ldots, g_{r}}(f)$ é $\mathcal{S}_{L_{1}, \ldots, L_{r}}\left(P_{n}\right)$, pois

$$
0 \neq P_{n}=\mathcal{S}_{L_{1}, \ldots, L_{r}}^{-1}\left(\mathcal{S}_{L_{1}, \ldots, L_{r}}\left(P_{n}\right)\right)
$$

Segue que

$$
\operatorname{mult}\left(\mathcal{S}_{g_{1}, \ldots, g_{r}}(f)\right)=\operatorname{mult}\left(\mathcal{S}_{L_{1}, \ldots, L_{r}}\left(P_{n}\right)\right)=\operatorname{mult}\left(P_{n}\right)=\operatorname{mult}(f) \neq \infty
$$

Logo, $\mathcal{S}_{g_{1}, \ldots, g_{r}}$ é injetora.

Mostremos agora que $\mathcal{S}_{g_{1}, \ldots, g_{r}}$ é sobrejetora. Para isto, é suficiente mostrar que sua imagem $A=k\left[\left[g_{1}, \ldots, g_{r}\right]\right]$ é fechada e densa em $\mathcal{R}$. Seja $P \in k\left[Y_{1}, \ldots, Y_{r}\right]$ um polinômio homogêneo qualquer. Consideremos

$$
Q=\mathcal{S}_{L_{1}, \ldots, L_{r}}^{-1}(P) \in \mathcal{R} \text {. }
$$

Logo, $P=S_{L_{1}, \ldots, L_{r}}(Q)$ é a forma inicial de $\mathcal{S}_{g_{1}, \ldots, g_{r}}(Q) \in A$. Pelo Lema 1.2 .6 , segue que $A$ é denso em $\mathcal{R}$.

Mostremos que $A$ é fechado em $\mathcal{R}$. Seja $h \in \mathcal{R}$ tal que

$$
h=\lim _{i \rightarrow \infty} f_{i}\left(g_{1}, \ldots, g_{r}\right),
$$

onde $f_{i} \in \mathcal{R}$. Devemos mostrar que $h \in A$.

Como $\mathcal{S}_{g_{1}, \ldots, g_{r}}$ é um homomorfismo e mult $\left(\mathcal{S}_{g_{1}, \ldots, g_{r}}(f)\right)=\operatorname{mult}(f)$, temos que

$$
\operatorname{mult}\left(f_{i}-f_{j}\right)=\operatorname{mult}\left(\mathcal{S}_{g_{1}, \ldots, g_{r}}\left(f_{i}\right)-\mathcal{S}_{g_{1}, \ldots, g_{r}}\left(f_{j}\right)\right) \text {. }
$$

Logo, $\left\{f_{i}\right\}$ é uma sequência de Cauchy em $\mathcal{R}$, e portanto existe $f \in \mathcal{R}$ tal que

$$
f=\lim _{i \rightarrow \infty} f_{i}
$$

Como $\mathcal{S}_{g_{1}, \ldots, g_{r}}$ é contínua segue que

$$
h=\lim _{i \rightarrow \infty} f_{i}\left(g_{1}, \ldots, g_{r}\right)=\mathcal{S}_{g_{1}, \ldots, g_{r}}\left(\lim _{i \rightarrow \infty} f_{i}\right)=\mathcal{S}_{g_{1}, \ldots, g_{r}}(f) \in A
$$




\subsection{O corpo das séries de Laurent}

Seja $k((X))$ o corpo de fraçōes do anel das séries de potências formais em uma variável $k[[X]]$.

Dado $h=f / g \in k((X)) \backslash\{0\}$, como podemos escrever $f=X^{n} u$ e $g=X^{m} v$, onde $n, m \in \mathbb{N}$ e $u, v$ são unidades em $k[[X]]$, temos que

$$
h=\frac{X^{n} u}{X^{m} v}=X^{n-m} u v^{-1}=X^{r} w
$$

onde $r \in \mathbb{Z}$ e $w$ é uma unidade em $k[[X]]$.

Isto mostra que todo elemento $h$ de $k((X))$ é da forma

$$
a_{-l} X^{-l}+a_{-l+1} X^{-l+1}+\ldots+a_{-1} X^{-1}+a_{0}+a_{1} X+\ldots
$$

onde $l \in \mathbb{N}$ e $a_{i} \in k, \forall i$. Os elementos de $k((X))$ são chamados de séries de potências formais de Laurent.

Dado $h \in k((X))$, escrito como acima, definimos a parte polar de $h$ como sendo

$$
\wp(h)=a_{-l} X^{-l}+a_{-l+1} X^{-l+1}+\ldots+a_{-1} X^{-1}
$$

Temos que $h-\wp(h) \in k[[X]]$. Escrevendo um elemento $h \in k((X)) \backslash\{0\}$ como $X^{r} w$, onde $r \in \mathbb{Z}$ e $w$ é uma unidade em $k[[X]]$, dizemos que $r$ é a multiplicidade de $h$ e denotamos por mult $(h)=r$. Também definimos mult $(0)=\infty$.

Analogamente a $k[[X]]$, definimos a distância entre $h, k \in k((X))$ como sendo

$$
d(h, k)= \begin{cases}\rho^{- \text {mult }(h-k)} & \text { se } h \neq k \\ 0 & \text { se } h=k\end{cases}
$$

onde $\rho>1$ é um número real. Observemos que $d(h, k) \leq 1$ se, e somente se, $\wp(h)=\wp(k)$. De fato, se $\wp(h)=\wp(k)$ então $h-k \in k[[X]]$ e mult $(h-k) \geq 0$. Logo

$$
d(h, k)=\rho^{- \text {mult }(h-k)}=\frac{1}{\rho^{\text {mult }(h-k)}} \leq 1
$$

pois $\rho>1$ e mult $(h-k) \geq 0$.

Reciprocamente, se $d(h, k) \geq 1$ então $\rho^{\text {mult }(h-k)} \geq 1$. Logo, mult $(h-k) \geq 0$ e, portanto, $\wp(h)=\wp(k)$.

Também podemos mostrar que o par $(k((X)), d)$ é um espaço métrico completo, como visto na Proposição 1.2.1.

Seja $\mathcal{F}=\left\{h_{\lambda} ; \lambda \in \Lambda\right\}$ uma família de elementos em $k((X))$, definimos

$$
\mathcal{F}^{+}=\left\{h_{\lambda}-\wp\left(h_{\lambda}\right) ; \lambda \in \Lambda\right\} \in k[[X]]
$$

Vamos dizer que $\mathcal{F}$ é somável em $k((X))$ se $\mathcal{F}^{+}$é somável em $k[[X]]$ e $\wp\left(h_{\lambda}\right)=0$, exceto possivelmente para um número finito de $\lambda^{\prime}$ s. Com esta definição, obtemos um resultado análogo à Proposição 1.2.3 sobre soma e produto de famílias somáveis em $k((X))$. 
Proposição 1.3.1 Se $T$ é um $k$-automorfismo de $k((X))$, então $T(X)=X u(X)$, onde $u \in k \|[X]]$, com $u(0) \neq 0$.

\section{Demonstração:}

Seja $T(X)=X^{r} u(X)$, onde $r \in \mathbb{Z}$ e $u$ é uma unidade em $k[[X]]$. Como $T$ é um homomorfismo, temos que

$$
T\left(X+X^{2}+\ldots\right)=T(X)+T\left(X^{2}\right)+\ldots=X^{r} u(X)+X^{2 r} u(X)^{2}+\ldots
$$

Se $r<0$ temos que $T\left(X+X^{2}+\ldots\right)$ não pertence a $k((X))$. Absurdo, pois $T$ é um.endomorfismo. Logo, $r \geq 0$. Assim, $T(X)=X^{r} u(X)$, onde $r \geq 0$ e $u$ é uma unidade em $k[[X]]$.

Como $T$ é um $k$-automorfismo, existe um $k$-automorfismo $T^{-1}$, definido por $T^{-1}(X)=X^{s} v(X)$, onde $s \geq 0$ e $v$ é uma unidade em $k[[X]]$, tal que

$$
X=T\left(T^{-1}(X)\right)=T\left(X^{s} v(X)\right)=\left(X^{s} v(X)\right)^{r} u\left(X^{s} v(X)\right)=X^{s r} v(X)^{r} u\left(X^{s} v(X)\right)=X^{s r} w(X),
$$

onde $w$ é uma unidade em $k[[X]]$. Logo, $w(X)=1$ e $X^{s r}=X$. Portanto, $s=r=1$.

Logo, $T(X)=X u(X)$, onde $u \in k[[X]]$, com $u(0) \neq 0$.

\subsection{Lema de Hensel}

Nesta seção vamos estabelecer um importante critério de redutibilidade em $k[[X]][Y]$, onde $k$ é um corpo qualquer e $X$ e $Y$ são indeterminadas. Definimos o grau do polinômio nulo como sendo $-\infty$.

Lema 1.4.1 Sejam p e q polinômios não constantes, relativamente primos em $k[Y]$, de graus respectivamente $r$ es. Dado um polinômio $F \in k[Y]$, tal que $\partial(F)<r+s$, existem polinômios unicamente determinados $g, h \in k[Y]$, com $\partial g<s$ e $\partial h<r$, tais que

$$
F=g p+h q
$$

\section{Demonstração:}

Como $p$ e $q$ são relativamente primos e. não constantes em $k[Y]$, segue que $\operatorname{mdc}(p, q)=1$, e portanto, existem polinômios $\varphi, \psi \in k[Y]$ tais que

$$
1=\varphi p+\psi q
$$

Assim,

$$
F=F \varphi p+F \psi q
$$

Do algoritmo da divisão segue que

$$
F \psi=p \rho+h
$$

$\operatorname{com} \rho, h \in k[Y]$ e $\partial h<\partial p=r$.

Substituindo (1.2) em (1.1), temos que

$$
F=F \varphi p+(p \rho+h) q=(F \varphi+\rho q) p+h q=g p+h q,
$$


onde $g=F \varphi+\rho q$. Assim, $g p=F-h q$ e

$$
\partial g+\partial p=\partial g p=\partial(F-h q)<r+s .
$$

Portanto, $\partial g<s$.

Mostremos agora a unicidade dos polinômios $g$ e $h$. Suponhamos que existam $g^{\prime}, h^{\prime} \in k[Y]$ tais que

$$
g p+h q=g^{\prime} p+h^{\prime} q
$$

com $\partial h, \partial h^{\prime}<r$ e $\partial g, \partial g^{\prime}<s$. Logo,

$$
\left(g-g^{\prime}\right) p=\left(h-h^{\prime}\right) q
$$

Como $p$ e $q$ são relativamente primos, então $p$ divide $h-h^{\prime}$. Como $\partial p=r>\partial\left(h-h^{\prime}\right)$, segue que $h-h^{\prime}=0$, o que por sua vez, implica que $g-g^{\prime}=0$, pois $p \neq 0$. Logo, $g$ e $h$ são unicamente determinados.

Teorema 1.4.2 (Lema de Hensel) Seja $f$ um polinômio mônico em $k[[X]][Y]$ tal que $f(0, Y)=$ $p(Y) q(Y)$, onde $p(Y)$ e $q(Y)$ são relativamente primos e não constantes em $k[Y]$ de graus $r$ e $s$, respectivamente. Então existem polinômios $g, h \in k[[X]][Y]$, unicamente determinados, de graus. (em $Y$ ) respectivamente $r$ e s, taiis que $f=g h$, com $g(0, Y)=p(Y)$ e $h(0, Y)=q(Y)$.

\section{Demonstração:}

Se $n=\partial_{Y} f$, então

$$
n=\partial(f(0, Y))=\partial(p(Y) q(Y))=r+s
$$

pois $f$ é mônico. Escreva

$$
f=f_{0}(Y)+X f_{1}(Y)+X^{2} f_{2}(Y)+\ldots
$$

onde $f_{0}(Y)=f(0, Y)$ tem grau $n$ e cada $f_{i}(Y), i \geq 1$, se não nulo, é um polinômio em $k[Y]$ de grau menor que $n$.

Queremos encontrar

$$
g(X, Y)=p(Y)+X g_{1}(Y)+X^{2} g_{2}(Y)+\cdots
$$

e

$$
h(X, Y)=q(Y)+X h_{1}(Y)+X^{2} h_{2}(Y)+\ldots
$$

onde os $g_{i}^{\prime}$ s, se não nulos, são polinômios em $k[Y]$ de grau menor que $r$ e os $h_{i}^{\prime}$ s, se não nulos, são polinômios em $k[Y]$ de grau menor que $s$, e são tais que $f=g h$. Logo,

$$
f_{i}(Y)=p(Y) h_{i}(Y)+g_{1}(Y) h_{i-1}(Y)+g_{2}(Y) h_{i-2}(Y)+\ldots+g_{i-1}(Y) h_{1}(Y)+g_{i}(Y) q(Y)
$$

No que segue, usaremos o lema anterior para obter os $g_{i}^{\prime}$ s e $h_{i}^{\prime} \mathrm{s}$ de maneira recursiva. Aplicando o Lema 1.4.1 para $f_{1}(Y)$ temos que existem $g_{1}, h_{1} \in k[Y]$, com $\partial\left(g_{1}\right)<r, \partial\left(h_{1}\right)<s$, tais que $f_{1}(Y)=p(Y) h_{1}(Y)+q(Y) g_{1}(Y)$. Devemos ter $f_{2}(Y)=p(Y) h_{2}(Y)+g_{1}(Y) h_{1}(Y)+q(Y) g_{2}(Y)$. 
Aplicando o Lema 1.4.1 para $f_{2}(Y)-g_{1}(Y) h_{1}(Y)$ encontramos $g_{2}$ e $h_{2}$. Suponhamos construídos $g_{j}$ e $h_{j}$ de graus respectivamente menores que $r$ e $s, \forall j \leq i-1$. Aplicando o Lema 1.4.1 a

$$
f_{i}(Y)-g_{1}(Y) h_{i-1}(Y)-g_{2}(Y) h_{i-2}(Y)-\ldots-g_{i-1}(Y) h_{1}(Y)
$$

encontramos $g_{i}$ e $h_{i}$ de graus respectivamente menores que $r$ e $s$, se não nulos.

Como a demonstração do Lema de Hensel é construtiva, é possivel determinar as séries $g$ e $h$ de forma recursiva.

Corolário 1.4.3 Seja $k$ um corpo algebricamente fechado e $u=u(X)$ uma unidade em $k[[X]]$. Se $n$ é um inteiro positivo que não é múltiplo da caracteristica de $k$, então existe um elemento invertivel $v$ em $k[[X]]$, tal que $u=v^{n}$.

\section{Demonstração:}

Como $u$ é invertível, segue que $u(0) \in k \backslash\{0\}$. Definindo

$$
f(X, Y)=Y^{n}-u(X) \in k[[X]][Y]
$$

temos que

$$
f(0, Y)=Y^{n}-u(0)=\prod_{i=1}^{n}\left(Y-a_{i}\right),
$$

onde os $a_{i}^{\prime} \mathrm{s}, i=1, \ldots, n$, são as raízes $n$-ésimas de $u(0)$, pois $k$ é algebricamente fechado. Observe que $f(0, Y)$ e $\frac{\partial(f)}{\partial Y}(0, Y)=n Y^{n-1}$ não possuem raízes em comum, pois $n$ não é múltiplo da característica de $k$. Logo, os $a_{i}^{\prime} \mathrm{s}$ são distintos.

Do Lema de Hensel, temos que existem $g_{1}, \ldots, g_{n} \in k[[X]][Y]$ tais que $g_{i}(0, Y)=Y-a_{i}$ e

$$
f=g_{1} \ldots g_{n}
$$

Como o grau de $f$ como polinômio em $Y$ é $n$, segue que o grau de cada $g_{i}$ em $Y$ é 1 . Assim

$$
g_{i}=Y=a_{i}(X)
$$

e $a_{i}(0)=a_{i} \neq 0$. Como $g_{1}\left(X, a_{1}(X)\right)=0$, segue que

$$
f\left(X, a_{1}(X)\right)=0 .
$$

Portanto,

$$
u=\left(a_{1}(X)\right)^{n} .
$$

O resultado segue tomando $v=a_{1}(X)$. 


\section{Capítulo 2}

\section{O Teorema da Preparação}

\subsection{O Teorema da Divisão}

Vamos continuar denotando por $\mathcal{R} \circ$ anel das séries de potências $k\left[\left[X_{1}, \ldots, X_{r}\right]\right]$ e por $\mathcal{M}_{\mathcal{R}}$ seu ideal maximal.

Definição 2.1.1 Seja $f \in \mathcal{R}$. Diremos que $f$ é regular de ordem $m$ com respeito a indeterminada $X_{j}$, se $f\left(0, \ldots, X_{j}, \ldots, 0\right)$ é divisivel por $X_{j}^{m}$, mas não é divisivel por $X_{j}^{m+1}$.

Diremos que $f$ é regular em $X_{j}$, se $f$ é regular com respeito a $X_{j}$ de ordem $n=$ mult $(f)$. Neste caso,

$$
\text { mult }(f)=\operatorname{mult}\left(f\left(0, \ldots, X_{j}, \ldots, 0\right)\right) \text {. }
$$

Lema 2.1.2 Dados $f, g \in \mathcal{M}_{\mathcal{R}}$ então fg é regular com respeito a $X_{j}$ de uma certa ordem se, e somente se, $f$ e $g$ são regulares com respeito a $X_{j}$ de determinadas ordens.

\section{Demonstração:}

Suponhamos que $f g$ é regular com respeito a $X_{j}$, ou seja,

$$
(f g)\left(0, \ldots, X_{j}, \ldots, 0\right)=X_{j}^{m} u\left(X_{j}\right)
$$

onde $u\left(X_{j}\right)$ é uma unidade em $k\left[\left[X_{j}\right]\right]$. Observemos que, como consequência, $f\left(0, \ldots, X_{j}, \ldots, 0\right) \neq 0$ e $g\left(0, \ldots, X_{j}, \ldots, 0\right) \neq 0$.

Se $f$ não é regular com respeito a $X_{j}$, então $f\left(0, \ldots, X_{j}, \ldots, 0\right)$ é uma unidade em $k\left[\left[X_{j}\right]\right]$. Logo, $f$ é uma unidade em $\mathcal{R}$, o que é uma contradição, pois $f \in \mathcal{M}_{\mathcal{R}}$. Analogamente, segue que $g$ é regular com respeito a $X_{j}$.

Reciprocamente, se $f$ e $g$ são regulares com respeito a $X_{j}$ de determinadas ordens, temos

$$
f\left(0, \ldots, X_{j}, \ldots, 0\right)=X_{j}^{m_{1}} u_{1}\left(X_{j}\right)
$$

e

$$
g\left(0, \ldots, X_{j}, \ldots, 0\right)=X_{j}^{m_{2}} u_{2}\left(X_{j}\right),
$$

onde $u_{1}\left(X_{j}\right)$ e $u_{2}\left(X_{j}\right)$ são unidades em $k\left[\left[X_{j}\right]\right]$. Logo,

$$
(f g)\left(0, \ldots, X_{j}, \ldots, 0\right)=X_{j}^{m_{1}+m_{2}}\left(u_{1} u_{2}\right)\left(X_{j}\right) .
$$


Assim, $f g$ é regular de ordem $m_{1}+m_{2}$ com respeito a $X_{j}$.

Vamos denotar $k\left[\left[X_{1}, \ldots, X_{r-1}\right]\right]$ por $\mathcal{R}^{\prime}$ e $\mathcal{M}_{\mathcal{R}^{\prime}}$ seu ideal maximal.

O próximo resultado, provado por Stickelberger em 1887, foi inspirado no Teorema da Preparação de Weierstrass de 1860 e tem como corolário o próprio Teorema da Preparação.

Teorema 2.1.3 (O Teorema da Divisão) Seja $F \in \mathcal{M}_{\mathcal{R}}$ regular de ordem $m$ com respeito a $X_{r}$. Dado $G \in \mathcal{R}$, existem $Q \in \mathcal{R}$ e $R \in \mathcal{R}^{\prime}\left[X_{r}\right]$, com $\partial_{X_{r}}(R)<m$, unicamente determinados por $F e$ $G$, tais que

$$
G=F Q+R
$$

\section{Demonstração:}

Escrevamos $G$ como um elemento de $\mathcal{R}^{\prime}\left[\left[X_{r}\right]\right]$

$$
G=\sum_{i=0}^{\infty} A_{i} X_{r}^{i}, \quad A_{i} \in \mathcal{R}^{\prime}, \forall i
$$

e seja

$$
R_{-1}=\sum_{i=0}^{m-1} A_{i} X_{r}^{i} \in \mathcal{R}^{\prime}\left[X_{r}\right]
$$

onde $\partial_{X_{r}}\left(R_{-1}\right)<m$.

Suponhamos que mult $(F)=n$ e escrevamos

$$
F=F_{n}+\ldots+F_{m}+F_{m+1}+\ldots
$$

onde $F_{i}$ é um polinômio homogêneo de grau $i$. Observemos que $n \leq m$.

Seja $P=F_{n}+\ldots+F_{m}$. Como $F$ é regular de ordem $m$ com respeito a $X_{r}$ temos que

$$
P=F_{n}+\ldots+F_{m}=c X_{r}^{m}+\sum_{i=0}^{m-1} B_{i} X_{r}^{i}, B_{i} \in \mathcal{M}_{\mathcal{R}^{\prime}}
$$

$\operatorname{com} c \in k \backslash\{0\}$.

Seja $H=G-R_{-1}=\sum_{i=m}^{\infty} A_{i} X_{r}^{i}$ e escrevamos

$$
H=H_{m}+H_{m+1}+\ldots
$$

sua decomposição em polinômios homogêneos.

Como $P=c X_{r}^{m}+\sum_{i=0}^{m-1} B_{i} X_{r}^{i}, B_{i} \in \mathcal{M}_{\mathcal{R}^{\prime}}$, existem $q_{0} \in k, R_{0} \in \mathcal{R}^{\prime}\left[X_{r}\right]$ tais que

$$
H_{m}=q_{0} P+R_{0}
$$

com $R_{0}=0$ ou $\partial_{X_{r}} R_{0}<m$.

Observemos que $q_{0}=0$ se, e somente se, $H_{m}\left(0, \ldots, 0, X_{r}\right)=0$. Se $q_{0}=0$ então $R_{0}=H_{m}$ e mult $\left(R_{0}\right)=m \geq n$. Se $q_{0} \neq 0$ então mult $\left(R_{0}\right)=n$. Portanto mult $\left(R_{0}\right) \geq n$. 
Temos que

$$
G=R_{-1}+H=R_{-1}+H_{m}+H_{m+1}+\ldots=R_{-1}+q_{0} P+R_{0}+H_{m+1}+\ldots
$$

Observando que $P=F-\left(F_{m+1}+\ldots\right)$ temos

$$
G=R_{-1}+R_{0}+q_{0} F-q_{0}\left(F_{m+1}+\ldots\right)+H_{m+1}+\ldots=R_{-1}+R_{0}+q_{0} F+\left(\dot{H}_{m+1}-q_{0} F_{m+1}\right)+\ldots
$$

Efetuando a divisão de $H_{m+1}-q_{0} F_{m+1}$ por $P$, obtemos

$$
H_{m+1}-q_{0} F_{m+1}=q_{1} P+R_{1}
$$

onde $q_{1} \in \mathcal{R}$ e $R_{1}=0$ ou $\partial_{X_{r}} R_{1}<m$. Notemos que mult $\left(q_{1}\right) \geq 1$.

Observemos que mult $\left(H_{m+1}-q_{0} F_{m+1}\right)=m+1$ ou mult $\left(H_{m+1}-q_{0} F_{m+1}\right)=\infty$. Se mult $\left(H_{m+1}-\right.$ $\left.q_{0} F_{m+1}\right)=m+1$ então

$$
m+1=\operatorname{mult}\left(q_{1} P+R_{1}\right) \leq \min \left\{\operatorname{mult}\left(q_{1}\right)+n, \text { mult }\left(R_{1}\right)\right\}
$$

Logo mult $\left(R_{1}\right)=m+1 \geq n+1>n$. Se mult $\left(H_{m+1}-q_{0} F_{m+1}\right)=\infty$ então $R_{1}=-q_{1} P$ e mult $\left(R_{1}\right)=$ mult $\left(q_{1}\right)+n \geq n+1>n$. Portanto, em ambos os casos, mult $\left(R_{1}\right) \geq n$.

Assim,

$$
\begin{gathered}
G=R_{-1}+R_{0}+q_{0} F+\left(q_{1} P+R_{1}\right)+\left(H_{m+2}-q_{0} F_{m+2}\right)+\ldots= \\
R_{-1}+R_{0}+R_{1}+\left(q_{0} F+q_{1} F\right)+\left(H_{m+2}-q_{0} F_{m+2}-q_{1} F_{m+1}\right)+\ldots
\end{gathered}
$$

Prosseguindo analogamente construímos $q_{0}, q_{1}, \ldots$ e $R_{0}, R_{1}, \ldots$, de modo que

$$
G=F\left(q_{0}+q_{1}+\ldots\right)+R_{-1}+R_{0}+R_{1}+\ldots
$$

Tome $Q=q_{0}+q_{1}+\ldots$ e $R=R_{-1}+R_{0}+R_{1}+\ldots$ em $\mathcal{R}^{\prime}\left[X_{r}\right]$, assim $G=F Q+R$.

Teorema 2.1.4 (Teorema da Preparação de Weierstrass) Seja $F \in \mathcal{R}$ regular de ordem $m$ com respeito a $X_{r}$. Então existem $U \in \mathcal{R}$ invertivel e $A_{1}, \ldots, A_{m} \in \mathcal{M}_{\mathcal{R}^{\prime}}$, unicamente determinados por $F$, tais que

$$
F U=X_{r}^{m}+A_{1} X_{r}^{m-1}+A_{2} X_{r}^{m-2} \ldots+A_{m} .
$$

Além disso, se $F$ é regular em $X_{r}$, isto é, se $m=m u l t(F)$, então $m u l t\left(A_{i}\right) \geq i, \forall i=1, \ldots, m$.

\section{Demonstração:}

Tomando $G=X_{r}^{m}$ no Teorema da Divisão, temos que existem $Q \in \mathcal{R}$ e $R \in \mathcal{R}^{\prime}\left[X_{r}\right]$, com $\partial_{X_{r}}(R)<m$, unicamente determinados por $F$ e $G$, tais que

$$
X_{r}^{m}=F Q+R
$$

Como $R \in \mathcal{R}^{\prime}\left[X_{r}\right]$ e $\partial_{X_{r}}(R)<m$, existem $A_{1}, \ldots, A_{m} \in \mathcal{R}^{\prime}$ tais que $-R=A_{1} X_{r}^{m-1}+A_{2} X_{r}^{m-2} \ldots+$ $A_{m}$. Tome $U=Q$. O fato de $U$ ser uma unidade segue da demonstração do Teorema da Divịsão, pois $G=X_{r}^{m}=H_{m}$, o que implica que $q_{0} \neq 0$. 
Temos que

$$
F\left(0, \ldots, 0, X_{r}\right) U\left(0, \ldots, 0, X_{r}\right)=X_{r}^{m}+A_{1}(0) X_{r}^{m-1}+A_{2}(0) X_{r}^{m-2} \ldots+A_{m}(0) .
$$

Mas $X_{r}^{m}$ divide $F\left(0, \ldots, 0, X_{r}\right)$ e portanto

$$
A_{1}(0)=A_{2}(0)=\ldots=A_{m}(0)=0 .
$$

Assim $A_{1}, \ldots, A_{m} \in \mathcal{M}_{\mathcal{R}^{\prime}}$.

Se $F$ é regular em $X_{r}$, então

$$
\text { mult }\left(X_{r}^{m}+A_{1} X_{r}^{m-1}+A_{2} X_{r}^{m-2} \ldots+A_{m}\right)=\operatorname{mult}(F U)=\operatorname{mult}(F)=m .
$$

Logo, mult $\left(A_{i}\right) \geq i, \forall i=1, \ldots, m$.

A unicidade segue da unicidade no Teorema da Divisão.

Corolário 2.1.5 (Teorema da Função Implícita) Seja $F \in \mathcal{M}_{\mathcal{R}}$ e tal que $\frac{\partial F}{\partial X_{r}}(0) \neq 0$. Então existe $\varphi\left(X_{1}, \ldots, X_{r-1}\right) \in \mathcal{M}_{\mathcal{R}^{\prime}}$ tal que $F\left(X_{1}, \ldots, X_{r-1}, \varphi\left(X_{1}, \ldots, X_{r-1}\right)\right)=0$ como um elemento de $\mathcal{R}^{\prime}$.

\section{Demonstração:}

Podemos escrever $F$ como um elemento de $\mathcal{R}^{\prime}\left[\left[X_{r}\right]\right]$,

$$
F=A_{0}+A_{1} X_{r}+A_{2} X_{r}^{2}+\ldots+A_{m} X_{r}^{m}+\ldots
$$

Como $F(0)=0$, segue que $A_{0} \in \mathcal{M}_{\mathcal{R}^{\prime}}$.

Temos que

$$
\frac{\partial F}{\partial X_{r}}=A_{1}+2 A_{2} X_{r} \ldots+m A_{m} X_{r}^{m-1}+\ldots
$$

Como $\frac{\partial F}{\partial X_{r}}(0) \neq 0$, temos que $A_{1}(0) \neq 0$.

Logo, $A_{1}$ é uma unidade em $\mathcal{R}^{\prime}$ e $F$ é regular de ordem 1 em $X_{r}$.

Segue do Teorema da Preparação que existem $U \in \mathcal{R}$ invertível e $B_{1} \in \mathcal{M}_{\mathcal{R}^{\prime}}$ unicamente determinados por $F$, tais que

$$
F U=X_{r}+B_{1}
$$

- O resultado segue tomando $\varphi\left(X_{1}, \ldots, X_{r-1}\right)=-B_{1}$, pois

$$
F\left(X_{1}, \ldots, X_{r-1},-B_{1}\right) U\left(X_{1}, \ldots, X_{r-1},-B_{1}\right)=0 .
$$

Como $U$ é invertível, temos que $F\left(X_{1}, \ldots, X_{r-1},-B_{1}\right)=0$.

A condição de $F$ ser regular não é tão restritiva quanto parece, pois se assumirmos que o corpo $k$ é infinito, mostraremos que compondo $F$ com um automorfismo linear de $\mathcal{R}$, transformamos $F$ numa série regular em uma das indeterminadas, que pode ser escolhida arbitrariamente.

Se $k$ é finito não podemos garantir a existência de $k$-automorfismos lineares, mas será sempre possível encontrar um $k$-automorfismo de $\mathcal{R}$ que transforma $F$ numa série de potências regular em uma das indeterminadas. 
Lema 2.1.6 Seja $k$ um corpo infinito. Dada uma familia finita $\mathcal{F}$ de polinômios homogêneos não nulos em $k\left[Y_{1}, \ldots, Y_{r}\right]$ existe uma transformação linear $T: k\left[X_{1}, \ldots, X_{r}\right] \rightarrow k\left[Y_{1}, \ldots, Y_{r}\right]$ tal que $\forall F \in \mathcal{F}$, de grau $n$, existe $c_{F} \in k \backslash\{0\}$ tal que

$$
F\left(T\left(X_{1}\right), \ldots, T\left(X_{r}\right)\right)=c_{F} X_{r}^{n}+\sum_{i=0}^{n-1} B_{i} X_{r}^{i}, B_{i} \in\left\langle X_{1}, \ldots, X_{r-1}\right\rangle \subset k\left[X_{1}, \ldots, X_{r-1}\right] .
$$

\section{Demonstração:}

Como $\mathcal{F}$ é finita e $k$ é infinito existe $\left(\alpha_{1}, \ldots, \alpha_{r}\right) \in k^{r}$ tal que

$$
F\left(\alpha_{1}, \ldots, \alpha_{r}\right) \neq 0, \forall F \in \mathcal{F} \text {. }
$$

De fato, como $\mathcal{F}$ é uma família finita de polinômios homogêneos em $k\left[Y_{1}, \ldots, Y_{r}\right]$ e cada um deles tem um número finito de raízes em $k^{r}$, podemos tomar $\alpha \in k^{r} \backslash\left\{\right.$ raízes de $F \in \mathcal{F}$ em $\left.k^{r}, \forall F\right\}$, pois $k$ é infinito.

Definimos $T$ por

$$
Y_{i}=T\left(X_{i}\right)=X_{i}+\alpha_{i} X_{r}, i=1, \ldots, r-1, Y_{r}=T\left(X_{r}\right)=\alpha_{r} X_{r}
$$

Portanto

$$
\begin{gathered}
Y_{1}^{m_{1}} \ldots Y_{r-1}^{m_{r-1}} Y_{r}^{m_{r}}=\left(X_{1}+\alpha_{1} X_{r}\right)^{m_{1}} \ldots\left(X_{r-1}+\alpha_{r-1} X_{r}\right)^{m_{r-1}}\left(\alpha_{r} X_{r}\right)^{m_{r}}= \\
\alpha_{1}^{m_{1}} \ldots \alpha_{r}^{m_{r}} X_{r}^{m_{1} \ldots m_{r}}+\sum_{i=0}^{m_{1} \ldots m_{r}-1} C_{i} X_{r}^{i}, C_{i} \in k\left[X_{1}, \ldots, X_{r-1}\right] .
\end{gathered}
$$

Então $\forall F \in \mathcal{F}$, de grau $n$, temos que

$$
F\left(T\left(X_{1}\right), \ldots, T\left(X_{r}\right)\right)=F\left(Y_{1}, \ldots, Y_{r}\right)=F\left(\alpha_{1}, \ldots, \alpha_{r}\right) X_{r}^{n}+\sum_{i=0}^{n-1} B_{i} X_{r}^{i}, B_{i} \in k\left[X_{1}, \ldots, X_{r-1}\right] .
$$

Tomando $c_{F}=F\left(\alpha_{1}, \ldots, \alpha_{r}\right) \neq 0$, temos que

$$
F\left(T\left(X_{1}\right), \ldots, T\left(X_{r}\right)\right)=c_{F} X_{r}^{n}+\sum_{i=0}^{n-1} B_{i} X_{r}^{i}, B_{i} \in k\left[X_{1}, \ldots, X_{r-1}\right] .
$$

Observemos que $B_{i}(0, \ldots, 0)=0$.

Corolário 2.1.7 Seja $k$ um corpo infinito. Dada uma família finita $\mathcal{F}$ de elementos não nulos em $\mathcal{M}_{\mathcal{R}}$, existe um automorfismo linear $\mathcal{T}$ de $\mathcal{R}$ tal que $\forall F \in \mathcal{F}, F \circ T$ é regular em $X_{r}$.

\section{Demonstração:}

Suponhamos $\mathcal{F}=\left\{F_{1}, \ldots, F_{n}\right\}$. Sejam $f_{1}, \ldots, f_{n}$ os polinômios homogêneos que definem a multiplicidade dos elementos de $\mathcal{F}$ respectivamente. Segue do Lema 2.1.6 que existe umá transformação linear $T_{1}: k\left[X_{1}, \ldots, X_{r}\right] \rightarrow k\left[X_{1}, \ldots, X_{r}\right]$ tal que $f_{i}\left(T_{1}\left(X_{1}\right), \ldots, T_{1}\left(X_{r}\right)\right)$ é regular em $X_{r}, \forall i=1, \ldots, n$.

Seja $T: \mathcal{R} \longrightarrow \mathcal{R} \cdot$ a aplicação de substituição $T=\mathcal{S}_{Y_{1}, \ldots, Y_{r}}$, onde

$$
Y_{i}=X_{i}+\alpha_{i} X_{r}, i=1, \ldots, r-1, Y_{r}=\alpha_{r} X_{r}
$$


e $\left(\alpha_{1}, \ldots, \alpha_{r}\right) \in k^{r}$ é tal que $f_{i}\left(\alpha_{1}, \ldots, \alpha_{r}\right) \neq 0, i=1, \ldots, n$. Notemos que $Y_{1}, \ldots, Y_{r}$ são linearmente independentes. Segue da Proposição 1.2.7 que $T$ é um automorfismo linear. Ainda

$$
T\left(F_{i}\right)=F_{i}\left(X_{1}+\alpha_{1} X_{r}, \ldots, \alpha_{r} X_{r}\right)=f_{i}\left(T_{1}\left(X_{1}\right), \ldots, T_{1}\left(X_{r}\right)\right)+\ldots
$$

Como $f_{i}\left(T_{1}\left(X_{1}\right), \ldots, T_{1}\left(X_{r}\right)\right)$ é regular em $X_{r}, \forall i=1, \ldots, n$, segue que $F_{i} \circ T$ é regular em $X_{r}$, $i=1, \ldots, n$.

O corolário seguinte é consequência dos principais resultados desta seção.

Corolário 2.1.8 Seja $k$ um corpo infinito. Seja $F \in \mathcal{R} \backslash\{0\}$ de multiplicidade n. Então existem um k-automorfismo $T$ de $\mathcal{R}$, uma unidade $U \in \mathcal{R}$ e $A_{1}, \ldots, A_{n} \in \mathcal{R}^{\prime}$ tais que mult $\left(A_{i}\right) \geq i$, para $i=1, \ldots, n, e$

$$
T(F) U=X_{r}^{n}+A_{1} X_{r}^{n-1}+\ldots+A_{n}
$$

\subsection{Fatoração no anel das séries de potências}

Nesta seção vamos estudar a fatoração no domínio $\mathcal{R}=k\left[\left[X_{1}, \ldots, X_{r}\right]\right]$ e mostraremos que $\mathcal{R}$ é um domínio de fatoração única. Vamos começar definindo duas classes de séries de potêneias que serão muito importantes: os pseudo-polinômios e os polinômios de Weierstrass.

Definição 2.2.1 Um pseudo-polinômio (resp. um polinômio de Weierstrass) em $X_{r}$ é uma série de potências.da forma

$$
P\left(X_{1}, \ldots, X_{r}\right)=X_{r}^{n}+A_{1} X_{r}^{n-1}+\ldots+A_{n} \in \mathcal{R}^{\prime}\left[X_{r}\right]
$$

onde $n \geq 1$ e mult $\left(A_{i}\right) \geq 1$ (resp. mult $\left.\left(A_{i}\right) \geq i\right)$, para $i=1, \ldots, n$.

Lema 2s2.2 Sejam $F_{1}, \ldots, F_{s}$ polinômios mônicos em $\mathcal{R}^{\prime}\left[X_{r}\right]$. Então $F_{1} \ldots F_{s}$ é um pseudo-polinômio (resp. um polinômio de Weierstrass) se, e somente se, cada $F_{i}, i=1, \ldots, s$, é um pseudo-polinômio (resp. um polinômio de Weierstrass).

\section{Demonstraçao:}

É suficiente considerar o caso $s=2$.

Sejam $F_{1}=X_{r}^{m}+A_{1} X_{r}^{m-1}+\ldots+A_{m}$ e $F_{2}=X_{r}^{n}+B_{1} X_{r}^{n-1}+\ldots+B_{n}$, onde $A_{i}, B_{j} \in \mathcal{R}^{\prime}$, $i=1, \ldots, m, j=1, \ldots, n$. Então

$$
\begin{aligned}
F_{1} F_{2}= & X_{r}^{m+n}+\left(A_{1}+B_{1}\right) X_{r}^{m+n-1}+\left(A_{2}+A_{1} B_{1}+B_{2}\right) X_{r}^{m+n-2}+\ldots+ \\
& +\left(A_{i}+A_{i-1} B_{1}+\ldots+A_{1} B_{i-1}+B_{i}\right) X_{r}^{m+n-i}+\ldots+A_{m} B_{n} .
\end{aligned}
$$

Se $F_{1}$ e $F_{2}$ são pseudo-polinômios (resp. polinômios de Weierstrass), então mult $\left(A_{i}\right) \geq 1$ (resp. mult $\left(A_{i}\right) \geq i$ ), para $i=1, \ldots, m$, e mult $\left(B_{i}\right) \geq 1$ (resp. mult $\left.\left(B_{i}\right) \geq i\right)$, para $i=1, \ldots, n$. Logo

mult $\left(A_{i}+A_{i-1} B_{1}+\ldots+A_{1} B_{i-1}+B_{i}\right) \geq \min \left\{\right.$ mult $\left(A_{i}\right)$, mult $\left(A_{i-1} B_{1}\right), \ldots$, mult $\left.\left(B_{i}\right)\right\}=$ $\min \left\{\operatorname{mult}\left(A_{i}\right)\right.$, mult $\left(A_{i-1}\right)$ mult $\left(B_{1}\right), \ldots$, mult $\left.\left(B_{i}\right)\right\} \geq 1$ (resp. $\left.\geq i\right)$. 
Portanto, $F_{1} F_{2}$ é um pseudo-polinômio (resp. um polinômio de Weierstrass).

Reciprocamente, se $F_{1} F_{2}$ é um pseudo-polinômio então $F_{1} F_{2}\left(0, \ldots, 0, X_{r}\right)=X_{r}^{m+n}$. Mas

$$
F_{1} F_{2}\left(0, \ldots, 0, X_{r}\right)=\left(X_{r}^{m}+A_{1}(0) X_{r}^{m-1}+\ldots+A_{m}(0)\right)\left(X_{r}^{n}+B_{1}(0) X_{r}^{n-1}+\ldots+B_{n}(0)\right) .
$$

Portanto, $A_{i}(0)=0, \forall i=1, \ldots, m$, e $B_{j}(0)=0, \forall j=1, \ldots, n$.

Logo, $F_{1}$ e $F_{2}$ são pseudo-polinômios.

Se $F_{1} F_{2}$ é um polinômio de Weierstrass então

$$
\text { mult }\left(F_{1} F_{2}\right)=\operatorname{mult}\left(F_{1} F_{2}\left(0, \ldots, 0, X_{r}\right)\right)=m+n \text {. }
$$

Mas mult $\left(F_{1} F_{2}\right)=\operatorname{mult}\left(F_{1}\right)+\operatorname{mult}\left(F_{2}\right)$. Como mult $\left(F_{1}\right) \leq m$ e mult $\left(F_{2}\right) \leq n$, segue que mult $\left(F_{1}\right)=m$ e mult $\left(F_{2}\right)=n$.

Logo, $F_{1}$ e $F_{2}$ são polinômios de Weierstrass.

Lema 2.2.3 Seja $F \in \mathcal{R}^{\prime}\left[X_{r}\right]$ um pseudo-polinômio. Então $F$ é redutível em $\mathcal{R}$ se, e somente se, $F$ é redutivel em $\mathcal{R}^{\prime}\left[X_{r}\right]$.

\section{Demonstração:}

Suponhamos que $F$ é redutível em $\mathcal{R}$. Então existem $F_{1}, F_{2} \in \mathcal{R}$, não unitários, tais que $F=F_{1} F_{2}$. Como $F$ é um pseudo-polinômio segue que $F$ é regular de uma certa ordem com respeito à $X_{r}$. Segue do Lema 2.1.2 que $F_{1}$ e $F_{2}$ são regulares de ordem $\geq 1$ com respeito a $X_{r}$. Do Teorema da Preparação de Weierstrass, temos que existem $U_{1}, U_{2} \in \mathcal{R}$ invertíveis, tais que $H_{1}=F_{1} U_{1}$ e $H_{2}=F_{2} U_{2}$ são pseudo-polinômios de grau $\geq 1$.

Seja $U=U_{1} U_{2}$. Temos que $U$ é uma unidade e $F U=F_{1} F_{2} U_{1} U_{2}=H_{1} H_{2}$.

Como $H_{1}$ e $H_{2}$ são pseudo-polinômios segue que $F U$ é um pseudo-polinômio. Mas $F .1=F$, e sendo $F$ um pseudo-polinômio, segue da unicidade do Teorema da Preparação que $F=H_{1} H_{2}$, ou seja, $F$ é redutível em $\mathcal{R}^{\prime}\left[X_{r}\right]$.

Reciprocamente, suponhamos que $F \in \mathcal{R}^{\prime}\left[X_{r}\right]$ é um pseudo-polinômio redutivel de grau $d$. Então existem $H_{1}$ e $H_{2}$ polinômios mônicos não unitários de graus respectivamente $m$ e $n$ em $\mathcal{R}^{\prime}\left[X_{r}\right]$, tais que $F=H_{1} H_{2}$, com $m+n=d$.

Pelo Lema 2.2.2, temos que $H_{1}$ e $H_{2}$ são pseudo-polinômios de grau $\geq 1$. Isto mostra que $H_{1}$ e $H_{2}$ não são invertíveis em $\mathcal{R}$. Então $F$ é redutível em $\mathcal{R}$.

Seja $A$ um domínio. Um elemento não nulo e não invertível $a \in A$ é primo, se toda vez que $a$ divide um produto de dois elementos de $A$ então ele divide um dos fatores. Um elemento $a \in A$ não nulo e não invertível é irredutível se $a=b c, \operatorname{com} b, c \in A$, implica que $b$ ou $c$ é uma unidade. Um domínio $A$ é um domínio de ideais principais (D.I.P.) se todo ideal próprio de $A$ é principal, ou seja, gerado por um único elemento. Dizemos que um domínio $A$ é um domínio de fatoração única (D.F.U.), se todo elemento nāo nulo e não invertível de $A$ se fatora como um produto de um número finito de elementos irredutíveis em $A$. Além disso, tal fatoração é única a menos da ordem dos fatores e elementos associados. No que segue precisaremos do seguinte resultado clássico da Álgebra, cuja demonstração pode ser encontrada, por exemplo, em [3], Teorema 2.3, pag. 182. 
Lema 2.2.4 (Lema de Gauss) Seja $A$ um domínio de fatoração única. Então $A[X]$ também é um dominio de fatoração única.

Teorema 2.2.5 $O$ anel $\mathcal{R}=k\left[\left[X_{1}, \ldots, X_{r}\right]\right]$ é um dominio de fatoração única.

\section{Demonstração:}

Já sabemos que $\mathcal{R}=k\left[\left[X_{1}, \ldots, X_{r}\right]\right]$ é um domínio, așsim basta mostrar que tódo elemento não nulo e não invertivel de $\mathcal{R}$ possui uma fatoração em finitos elementos irredutiveis e que todo elemento irredutível de $\mathcal{R}$ é primo (ver [6], Teorema 5, pag. 150). A prova será por indução sobre $r$.

Primeiramente, mostremos que $k[[X]]$ é um domínio de fatoração única. Sejam $I$ um ideal próprio de $k[[X]] \mathrm{e}$

$$
A=\left\{m \in \mathbb{N} \backslash\{0\} ; X^{m} \mid f, \forall f \in I\right\} .
$$

Temos que $A \neq \emptyset$, pois $1 \in A$. Além disso, $A$ possui um máximo. De fato, seja $f=X^{m_{0}} u$, onde $u$ é uma unidade. Então, $X^{m_{0}+1}$ não divide $f$. Logo, $m_{0}+1$ é um limitante superior para $A$ e, portanto, $A$ tem ưm máximo.

Seja $n=\max A$. Vamos mostrar que $I=\left\langle X^{n}\right\rangle$. Seja $f \in I$, então $X^{n}$ divide $f$. Logo, $f=X^{n} g$ e, portanto, $I \subset\left\langle X^{n}\right\rangle$. Por outro lado, como $n=\max A$, existe $h \in I$ tal que $X^{n}$ divide $h$ mas $X^{n+1}$ não divide $h$. Logo, $\dot{h}=X^{n} u$, onde $u$ é uma unidade. Então, $X^{n}=h u^{-1}$ e, portanto, $\left\langle X^{n}\right\rangle \subset I$.

Portanto, $k[[X]]$ é um domínio de ideais principais e logo é um domínio de fatoração única (ver [3], Teorema 5.2, pag. 112).

Suponhamos por hipótese de indução que $\mathcal{R}^{\prime}$ é um D.F.U.. Sejam $F, G, H \in \mathcal{R} \operatorname{com} F$ irredutível e $F \mid G H$. Então, $F$ também é irredutivel em $\mathcal{R}^{\prime}\left[X_{r}\right]$ e, portanto, $F$ é primo em $\mathcal{R}^{\prime}\left[X_{r}\right]$, que é um D.F.U., pelo Lema de Gauss. Queremos mostrar que $F \mid G$ ou $F \mid H$. Como $G H=F B$, para algum $B \in \mathcal{R}$, se $G$ é invertível em $\mathcal{R}$, entāo $H=F B G^{-1}$. Logo, $F \mid H$. Analogamente, se $H$ é invertível em $\mathcal{R}$, então $F \mid G$. Se $G$ e $H$ não são invertíveis em $\mathcal{R}$, então pelo Teorema de Preparação da Weierstrass, $F, G$ e $H$ são associados a polinômios de Weierstrass. Considerando então $F, G$ e $H$ como elementos de $\mathcal{R}^{\prime}\left[X_{r}\right]$, como $F$ é primo em $\mathcal{R}^{\prime}\left[X_{r}\right]$, segue que $F \mid G$ ou $F \mid H$.

Logo, $\mathcal{R}$ é um domínio de fatoração única.

Corolário 2.2.6 Suponhamos que $F \in \mathcal{R}^{\prime}\left[X_{r}\right]$ seja um pseudo-polinômio (resp. um polinômio de Weierstrass) com respeito à indeterminada $X_{r}$. Se $F=F_{1} \ldots F_{s}$ é a decomposição de $F$ em fatores irredutiveis em $\mathcal{R}$, então podemos escolher uma decomposição onde cada $F_{i}$ é um pseudo-polinômio (resp. um polinômio de Weierstrass).

\section{Demonstração:}

Se $F=F_{1} \ldots F_{s}$ é a decomposição de $F$ em fatores irredutiveis em $\mathcal{R}$, então $F=F_{1} \ldots F_{s}$ é também a decomposição de $F$ em fatores irredutiveis em $\mathcal{R}^{\prime}\left[X_{r}\right]$. Como $F$ é um pseudo-polinômio (resp. um polinômio de Weierstrass), portanto um polinômio mônico, podemos supor que os $F_{i}^{\prime}$ s são mônicos. Então $F_{1}, \ldots, F_{s}$ são pseudo-polinômios (resp. um polinômio de Weierstrass), pelo Lema 2.2 .2 . 


\subsection{Teorema da base de Hilbert-Rückert}

Um anel $A$ é Noetheriano se todo ideal de $A$ é finitamente gerado.

Seja $f=a_{n} X^{n}+\ldots+a_{1} X+a_{0} \in A[X]$. Chamamos $a_{n} X^{n}$ de termo líder de $f$ e $a_{n}$ de coeficiente líder de $f$.

Teorema 2.3.1 (Teorema da Base de Hilbert) Se A é um anel Noetheriano, então $A[X]$ é Noetheriano.

\section{Demonstração:}

Seja $I$ um ideal de $A[X]$, queremos mostrar que $I$ é finitamente gerado. Escolha um elemento $f_{1}$ de $I$ de grau mínimo. Se $I=\left\langle f_{1}\right\rangle$, o resultado está provado. Caso contrário, escolha $f_{2} \in I \backslash\left\langle f_{1}\right\rangle$ de menor grau. Se $I=\left\langle f_{1}, f_{2}\right\rangle$, o resultado segue. Senão, escolha $f_{3} \in I \backslash\left\langle f_{1}, f_{2}\right\rangle$ de menor grau. Se $I=\left\langle f_{1}, f_{2}, f_{3}\right\rangle$, o resultado segue. Senão, continuamos o processo. Mostraremos que este processo para após um número finito de passos. Seja $J$ o ideal em $A$ gerado pelo coeficientes líderes $a_{i}^{\prime} \mathrm{s} \operatorname{dos} f_{i}^{\prime} \mathrm{s}$ escolhidos acima. Como $A$ é um anel Noetheriano, podemos supor que $J=\left\langle a_{1}, \ldots, a_{m}\right\rangle$ para algum $m \in \mathbb{N}$.

Mostremos que $I=\left\langle f_{1}, \ldots, f_{m}\right\rangle$. Suponhamos, por absurdo, que $I \neq\left\langle f_{1}, \ldots, f_{m}\right\rangle$. Consideremos o elemento $f_{m+1} \in I \backslash\left\langle f_{1}, \ldots, f_{m}\right\rangle$, o qual sabemos ser de menor grau, por construção. Temos que

$$
a_{m+1}=\sum_{i=1}^{m} c_{i} a_{i} ; c_{i} \in A .
$$

Por construção, $\partial\left(f_{m+1}\right) \geq \partial\left(f_{i}\right), i=1, \ldots, m$. Então

$$
g=\sum_{i=1}^{m} c_{i} f_{i} X^{\partial\left(f_{m+1}\right)-\partial\left(f_{i}\right)} \in\left\langle f_{1}, \ldots, f_{m}\right\rangle .
$$

Como o coeficiente líder de $g$ é igual ao coeficiente líder de $f_{m+1}$, segue que o grau de $f_{m+1}-g$ é menor que o grau de $f_{m+1}$ e, além disso

$$
f_{m+1}-g \in I \backslash\left\langle f_{1}, \ldots, f_{m}\right\rangle,
$$

o que é uma contradição.

Portanto, $I=\left\langle f_{1}, \ldots, f_{m}\right\rangle$ e $A[X]$ é Noetheriano.

Vamos usar o Teorema da Base de Hilbert e o Teorema de Divisão para provar o seguinte resultado

Teorema 2.3.2 (Teorema da Base de Rückert) $O$ anel $\mathcal{R}=k\left[\left[X_{1}, \ldots, X_{r}\right]\right]$ é Noetheriano.

\section{Demonstração:}

A prova será feita por indução sobre $r$.

Sabemos que $k[[X]]$ é um domínio de ideais principais, e portanto, é um anel Noetheriano.

Suponhamos, por hipótese de indução, que $\mathcal{R}^{\prime}=k\left[\left[X_{1}, \ldots, X_{r-1}\right]\right]$ é Noetheriano para $r \geq 2$. Seja $I \subset \mathcal{R}$ um ideal próprio não nulo e seja $G \in I \backslash\{0\}$ um elemento qualquer. Após uma mudança de coordenadas, se necessário, podemos supor $G$ regular com respeito a $X_{r}$. 
Como $\mathcal{R}^{\prime}$ é Noetheriano, segue do Teorema da Base de Hilbert que $\mathcal{R}^{\prime}\left[X_{r}\right]$ é Noetheriano.

Além disso, existem $G_{1} \ldots, G_{m} \in \mathcal{R}^{\prime}\left[X_{r}\right]$, tais que

$$
I \cap \mathcal{R}^{\prime}\left[X_{r}\right]=\left\langle G_{1}, \ldots, G_{m}\right\rangle
$$

Seja $F \in I$, segue do Teorema da Divisão que podemos escrever

$$
F=g G+R ; g \in \mathcal{R} \in R \in \mathcal{R}^{\prime}\left[X_{r}\right]
$$

Como $F, G \in I$, segue que $R \in I$ e, portanto, $R \in I \cap \mathcal{R}^{\prime}\left[X_{r}\right]$. Logo, existem $g_{1}, \ldots, g_{m} \in$ $\mathcal{R}^{\prime}\left[X_{r}\right] \subset \mathcal{R}$ tais que

$$
R=g_{1} G_{1}+\ldots+g_{m} G_{m}
$$

Segue que

$$
F=g G+g_{1} G_{1}+\ldots+g_{m} G_{m} .
$$

Portanto, $F \in\left\langle G, G_{1}, \ldots, G_{m}\right\rangle$ e assim $I=\left\langle G, G_{1}, \ldots, G_{m}\right\rangle$.

\subsection{Eliminação}

Sejam $A$ um D.F.U. e $f=a_{0} Y^{n}+a_{1} Y^{n-1}+\ldots+a_{n}$ e $g=b_{0} Y^{m}+b_{1} Y^{m-1}+\ldots+b_{m}$ polinômios em $A[Y]$. Queremos determinar um critério para decidir quando os polinômios $f$ e $g$ possuem fator comum não constante em $A[Y]$. Para tanto, definimos a resultante de $f$ e $g$, que denotamos por $R_{Y} \cdot(f, g)$, e é dada por

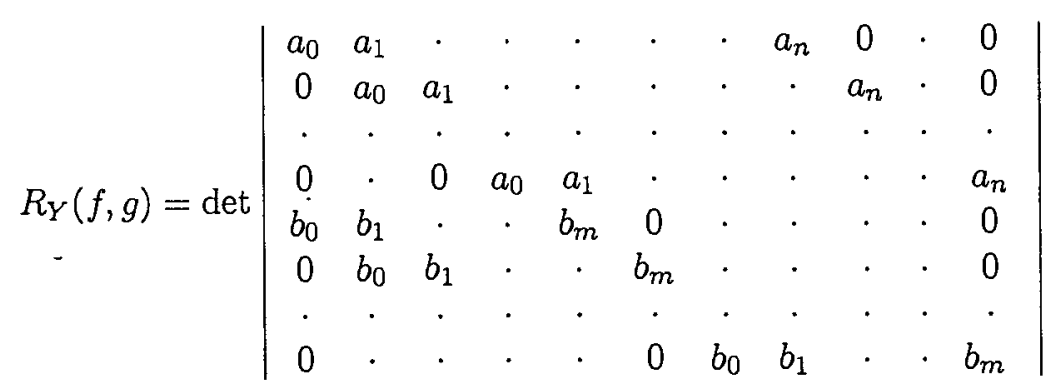

Lema 2.4.1 Os polinômios $f$ e $g$ em $A[Y]$ admitem fator comum não constante se, e somente se, existem polinômios não nulos $p, q \in A[Y]$, de graus menores que os graus de $f$ e g respectivamente, tais que $q f=p g$.

\section{Demonstração:}

Suponhamos que $h$ é um fator comum não constante de $f$ e $g$, ou seja, existem $p, q \in A[Y]$ tais que $f=h p$ e $g=h q$. Então $q f=q h p$ e $p g=p h q$, portanto $q f=p g$, pois $A[Y]$ é um anel comutativo.

Reciprocamente, suponhamos que existam polinômios não nulos $p, q \in A[Y]$, tais que $q f=p g$, com $\partial p<\partial f$ e $\partial q<\partial g$. Se $f$ e $g$ não têm fator comum não constante, então $f$ divide $p$, pois $A[Y]$ é um D.F.U.. Portanto, $\partial f \leq \partial p$, o que é um absurdo. Logo $f$ e $g$ admitem fator comum não constante. 
Proposição 2.4.2 Sejam $f=a_{0} Y^{n}+a_{1} Y^{n-1}+\ldots+a_{n}$ e $g=b_{0} Y^{m}+b_{1} Y^{m-1}+\ldots+b_{m}$ polinômios não constantes em $A[Y]$, de graus $n$ e $m$ respectivamente. ${ }^{\prime}$ Então $R_{Y}(f, g)=0$ se, e somente se, $f$ e $g$ admitem fator comum não constante em $A[Y]$.

\section{Demonstração:}

Segue do lema acima que $f$ e $g$ admitem fator comum não constante em $A[Y]$ se, e somente se, existem polinômios não nulos $p, q \in A[Y]$, com $\partial p<n$ e $\partial q<m$, tais que $q f$. $=p g$. Escrevendo $p=c_{1} Y^{n-1}+c_{2} Y^{n-2}+\ldots+c_{n}$ e $q=d_{1} Y^{m-1}+d_{2} Y^{m-2}+\ldots+d_{m}$, temos

$$
d_{1} Y^{m-1} f+d_{2} Y^{m-2} f+\ldots+d_{m} f-c_{1} Y^{n-1} g-c_{2} Y^{n-2} g-\ldots-c_{n} g=0 .
$$

Como os $c_{i}^{\prime}$ s e $d_{i}^{\prime}$ s não são todos nulos, $Y^{m-1} f, Y^{m-2} f, \ldots Y f, f, Y^{n-1} g, Y^{n-2} g, \ldots, Y g, g$ são linearmente dependentes no corpo de frações $F$ de $A$.

Escrevendo os polinômios acima como vetores em $F^{n+m}$, temos que o determinante da matriz $R$ cujas linhas são esses vetores é $R_{Y}(f, g)$. Como esses vetores são linearmente dependentes, segue que $R_{Y}(f, g)=0$. Reciprocamente, se $\operatorname{det} R=0$, então os vetores são linearmente dependentes e portanto, $f$ e $g$ têm fator comum não constante em $A[Y]$.

Corolário 2.4.3 Sejam $P, Q \in \mathcal{R}[Y]$ pseudo-polinômios com respeito à indeterminada $Y$. As séries $P$ e $Q$ admitem fator comum não unitário em $\mathcal{R}[[Y]]$ se, e somente se, $R_{Y}(P, Q)=0$.

\section{Demonstração:}

Do Corolário 2.2.6 temos que $P$ e $Q$ admitem fator comum não unitário em $\mathcal{R}[[Y]]$ se, e somente se, $P$ e $Q$ admitem fator comum não unitário em $\mathcal{R}[Y]$. O resultado segue agora da Proposição 2.4.2, lembrando que $\mathcal{R}$ é um D.F.U..

Corolário 2.4.4 Consideremos $\mathbb{C}$ o corpo dos número complexos e $A=\mathbb{C}\{X\}$ que é o anel das séries de potências absolutamente convergentes numa vizinhança da origem, onde $X=\left(X_{1}, \ldots, X_{n-1}\right)$. Sejam $f=a_{0}(X) Y^{n}+a_{1} Y^{n-1}+\ldots+a_{n}(X)$ e $g=b_{0}(X) Y^{m}+b_{1}(X) Y^{m-1}+\ldots+b_{m}(X)$ polinômios em $\mathbb{C}\{X\}[Y]$ e seja $U$ uma vizinhança do zero em $\mathbb{C}^{n-1}$ onde os $a_{i}^{\prime} s$ e $b_{j}^{\prime}$ s convergem absolutamente. Denotando por $R(X)$ a resultante de $f$ e $g$, temos que $R(\alpha)=0, \alpha \in U$, se, e somente se, $a_{0}(\alpha) .=$ $b_{0}(\alpha)=0$ ou $f(\alpha, Y)$ e $g(\alpha, Y)$ admitem uma raiz comum em $\mathbb{C}$.

Proposição 2.4.5 Sejam $f=a_{0} Y^{n}+a_{1} Y^{n-1}+\ldots+a_{n}$ e $g=b_{0} Y^{m}+b_{1} Y^{m-1}+\ldots+b_{m}$ polinômios não nulos em $A[Y]$ de graus $n$ e $m$ respectivamente. Então existem polinômios não nulos $p, q \in A[Y]$, com $\partial p<n$ e $\partial q<m$, tais que.

$$
q f+p g=R_{Y}(f, g)
$$

\section{Demonștração:}

Se $f$ e $g$ admitem fator comum não constante então $R_{Y}(f, g)=0$. Além disso, segue do Lema 2.4.1, que existem polinômios não nulos $p, q \in A[Y], \operatorname{com} \partial p<n$ e $\partial q<m$, tais que $q f=p g$. Então

$$
q f+(-p g)=0=R_{Y}(f, g) .
$$


Suponhamos que $f$ e $g$ não têm fator comum não constante. Da Proposição 2.4 .2 segue que $R_{Y}(f, g) \neq 0$. Por outro lado temos que

$$
\left(\begin{array}{ccccccccccc}
a_{0} & a_{1} & \cdot & \cdot & \cdot & \cdot & \cdot & a_{n} & 0 & \cdot & 0 \\
0 & a_{0} & a_{1} & \cdot & \cdot & \cdot & \cdot & \cdot & a_{n} & \cdot & 0 \\
\cdot & \cdot & \cdot & \cdot & \cdot & \cdot & \cdot & \cdot & \cdot & \cdot & \cdot \\
0 & \cdot & 0 & a_{0} & a_{1} & \cdot & \cdot & \cdot & \cdot & \cdot & a_{n} \\
b_{0} & b_{1} & \cdot & \cdot & b_{m} & 0 & \cdot & \cdot & \cdot & \cdot & 0 \\
0 & b_{0} & b_{1} & \cdot & \cdot & b_{m} & \cdot & \cdot & \cdot & \cdot & \cdot \\
\cdot & \cdot & \cdot & \cdot & \cdot & \cdot & \cdot & \cdot & \cdot & \cdot & \cdot \\
0 & \cdot & \cdot & \cdot & \cdot & 0 & b_{0} & b_{1} & \cdot & \cdot & \cdot \\
b_{m}
\end{array}\right)\left(\begin{array}{c}
Y^{n+m-1} \\
Y^{n+m-2} \\
\vdots \\
Y^{n} \\
Y^{n-1} \\
Y^{n-2} \\
\vdots \\
1
\end{array}\right)=\underbrace{\left(\begin{array}{c}
Y^{m-1} f \\
Y^{m-2} f \\
\vdots \\
f \\
Y^{n-1} g \\
Y^{n-2} g \\
\vdots \\
g
\end{array}\right)}_{(*)}
$$

Trocando a última coluna da matriz cujo determinante é $R_{Y}(f, g)$ por (*), obtemos a matriz

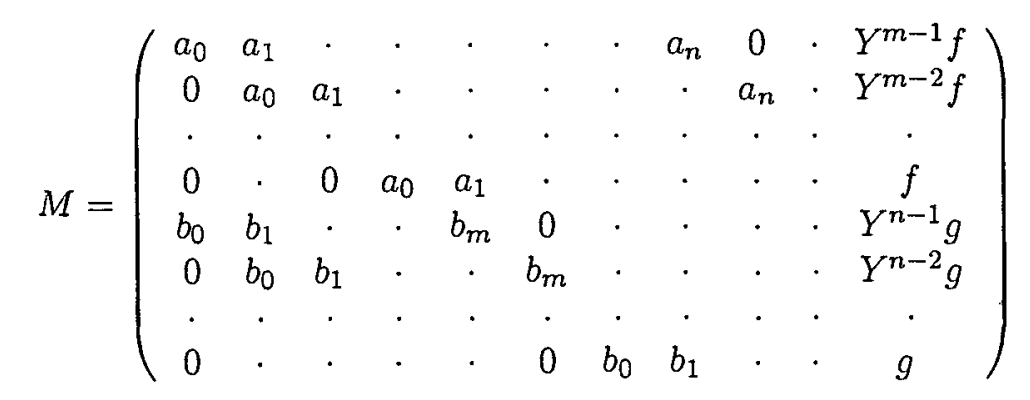

Aplicando a Regra de Cramer (ver [4], pag. 151) ao sistema acima com coeficientes no corpo de frações de $A$, obtemos

$$
\frac{\operatorname{det} M}{R_{Y}(f, g)}=1 \text {. }
$$

Calculando o determinante de $M$, desenvolvendo-o pela última coluna, temos que

$$
\begin{gathered}
\operatorname{det} M=A_{1} Y^{m-1} f+A_{2} Y^{m-2} f+\ldots+A_{m} f+B_{1} Y^{n-1} g+B_{2} Y^{n-2} g+\ldots+B_{n} g \\
=\left(A_{1} Y^{m-1}+A_{2} Y^{m-2}+\ldots+A_{m}\right) f+\left(B_{1} Y^{n-1}+B_{2} Y^{n-2}+\ldots+B_{n}\right) g
\end{gathered}
$$

onde $A_{i}=(-1)^{m+n+i}$.(determinante da matriz obtida de $M$ retirando-se a $i$-ésima linha e a última coluna), $i=1, \ldots, m, \mathrm{e} \cdot B_{j}=(-1)^{m+n+j}$. (determinante da matriz obtida de $M$ retirando-se a $j-$ ésima linha e a última coluna), $j=m+1, \ldots, m+n$.

$O$ resultado segue tomando

$$
q=A_{1} Y^{m-1}+A_{2} Y^{m-2}+\ldots+A_{m} \text { e } p=B_{1} Y^{n-1}+B_{2} Y^{n-2}+\ldots+B_{n}
$$

pois $R_{Y}(f, g)=\operatorname{det} M$.

Sejam $\mathrm{X}=\left(X_{1}, \ldots, X_{n}\right)$ e $\mathrm{Y}={ }^{\cdot}\left(Y_{1}, \ldots, Y_{m}\right)$ dois conjuntos de indeterminadas e consideremos o anel

$$
\mathcal{R}^{\prime \prime}=A[\mathrm{X}, \mathrm{Y}]=A\left[X_{1}, \ldots, X_{n}, Y_{1}, \ldots, Y_{m}\right] .
$$

Consideremos os polinômios

$$
F=a_{0}\left(\dot{Z}-X_{1}\right) \ldots\left(Z-X_{n}\right) \in \mathcal{R}^{\prime \prime}[Z]
$$


e

$$
G=b_{0}\left(Z-Y_{1}\right) \ldots\left(Z-Y_{m}\right) \in \mathcal{R}^{\prime \prime}[Z]
$$

A resultante $R_{Z}(F, G)$ é, portanto, um polinômio em $\mathcal{R}^{\prime \prime}$.

Lema 2.4.6 O polinômio $R_{Z}(F, G)$ é homogêneo de grau $\partial F \partial G=n m$ em $\mathcal{R}^{\prime \prime}$.

\section{Demonstração:}

Podemos escrever

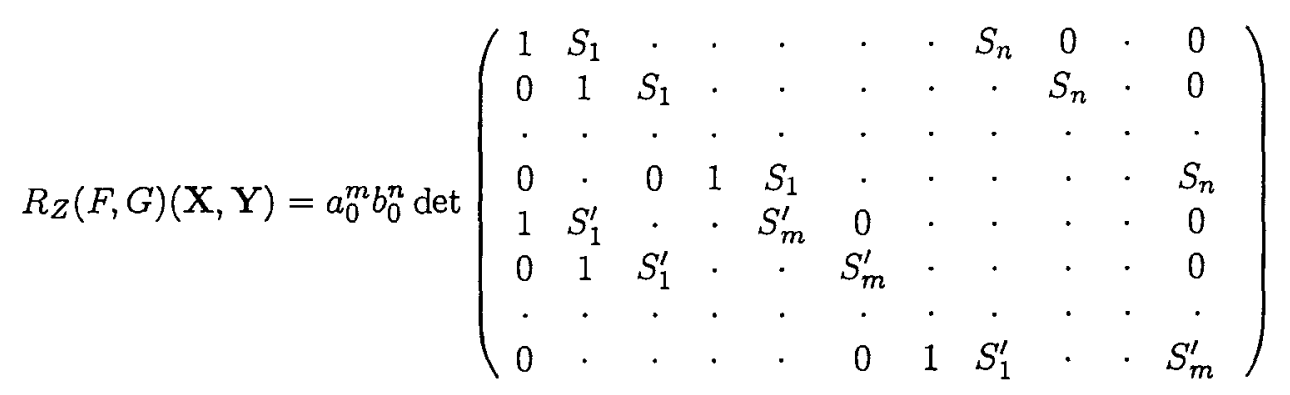

onde os $S_{i}$ 's e os $S_{j}^{\prime}$ 's são as funções simétricas elementares nas indeterminadas $\mathrm{X}$ e $\mathrm{Y}$, respectivamente:

$$
S_{1}=(-1)\left(X_{1}+\ldots+X_{n}\right), S_{2}=(-1)^{2} \sum_{\substack{i, j=1 \\ i \neq j}}^{n} X_{i} X_{j}, \ldots, S_{n}=(-1)^{n}\left(X_{1} \ldots X_{n}\right) .
$$

Substituindo, na matriz acima, $X_{i}$ por $T^{i} X_{i}, i=1, \ldots, n$, e $Y_{j}$ por $T^{j} Y_{j}, j=1, \ldots, m$, obtemos

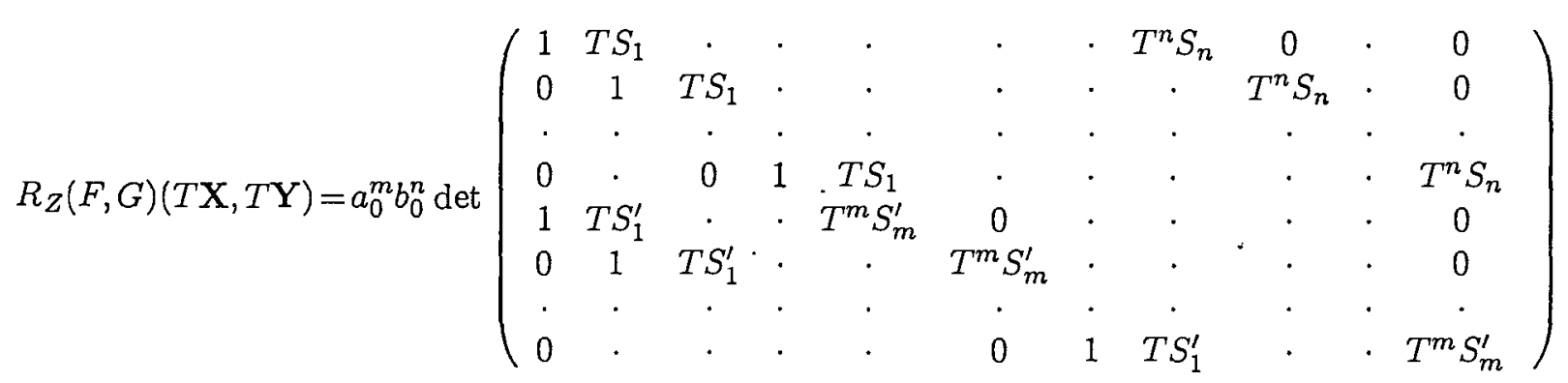

Multiplicando, na matriz acima, a segunda linha por $T$, a terceira linha por $T^{2}, \ldots$, a $m$-ésima linha por $T^{m-1}$, a $(m+2)$-ésima linha por $T, \ldots$, a $(m+n)$-ésima linha por $T^{n-1}$, obtemos

$$
T^{M} R_{Z}(F, G)(T \mathbf{X}, T \mathrm{Y})=T^{N} R_{Z}(F, G)(\mathbf{X}, \mathbf{Y}),
$$

onde $M=(1+2+\ldots+m-1)+(1+2+\ldots+n-1)$ e $N=1+\ldots+(m+n-1)$. Portanto

$$
R_{Z}(F, G)(T \mathbf{X}, T \mathbf{Y})=T^{N-M} R_{Z}(F, G)(\mathbf{X}, \mathbf{Y})
$$

$\operatorname{com} N-M=m n$.

Logo, $R_{Z}(F, G)$ é um polinômio homogêneo de grau $n m$ em $\mathcal{R}^{\prime \prime}$.

Vamos usar o lema acima para provar o seguinte resultado. 
Proposição 2.4.7 Sejam $f=a_{0} Y^{n}+a_{1} Y^{n-1}+\ldots+a_{n}$ e g $=b_{0} Y^{m}+b_{1} Y^{m-1}+\ldots+b_{m}$ polinômios não constantes em $k[Y]$, de graus $n$ e $m$ respectivamente, onde $k$ é um corpo. Seja $E$ uma extensão de $k$ que contém as raízes $\alpha_{1}, \ldots, \alpha_{n}$ e $\beta_{1}, \ldots, \beta_{m}$ de $f$ e $g$ respectivamente. Entāo

$$
R_{Y}(f, g)=a_{0}^{m} b_{0}^{n} \prod_{i=1}^{n} \prod_{j=1}^{m}\left(\alpha_{i}-\beta_{j}\right)=a_{0}^{m} \prod_{i=1}^{n} g\left(\alpha_{i}\right)=(-1)^{n m} b_{0}^{n} \prod_{j=1}^{m} f\left(\beta_{j}\right) .
$$

\section{Demonstração:}

Consideremos os polinômios $F, G$ e $R_{Z}(F, G)$, onde

$$
F=a_{0}\left(Z-X_{1}\right) \ldots\left(Z-X_{n}\right) \in \mathcal{R}^{\prime \prime}[Z]
$$

$\mathrm{e}$

$$
G=b_{0}\left(Z-Y_{1}\right) \ldots\left(Z-Y_{m}\right) \in \mathcal{R}^{\prime \prime}[Z] .
$$

Tomando $X_{i}=Y_{j}$, temos que $F$ e $G$ possuem fator comum não constante. Logo, $R_{Z}(F, G)$ se anula quando $X_{i}=Y_{j}$. Assim, $R_{Z}(F, G)$ como um polinômio em $\mathcal{R}^{\prime \prime}[Z]$ é divisível por $X_{i}-Y_{j}, \forall i, j$.

Como $X_{i}-Y_{j}$ e $X_{r}-Y_{s}$ são relativamente primos $\forall(i, j) \neq(r, s)$, temos que $R_{Z}(F, G)$ é divisível por

$$
P=a_{0}^{m} b_{0}^{n} \prod_{i=1}^{n} \prod_{j=1}^{m}\left(X_{i}-Y_{j}\right)
$$

que é um polinômio homogêneo em $\mathcal{R}^{\prime \prime}[Z]$ de grau $m n$, o mesmo grau de $R_{Z}(F, G)$.

O termo de menor grau em $R_{Z}(F, G)$ que só contém as indeterminadas de $\mathrm{Y}$ é $a_{0}^{m} b_{0}^{n}\left(Y_{1} \ldots Y_{m}\right)^{n}=$ $a_{0}^{m} b_{0}^{n} S_{m}^{\prime}$, que corresponde à diagonal principal da matriz

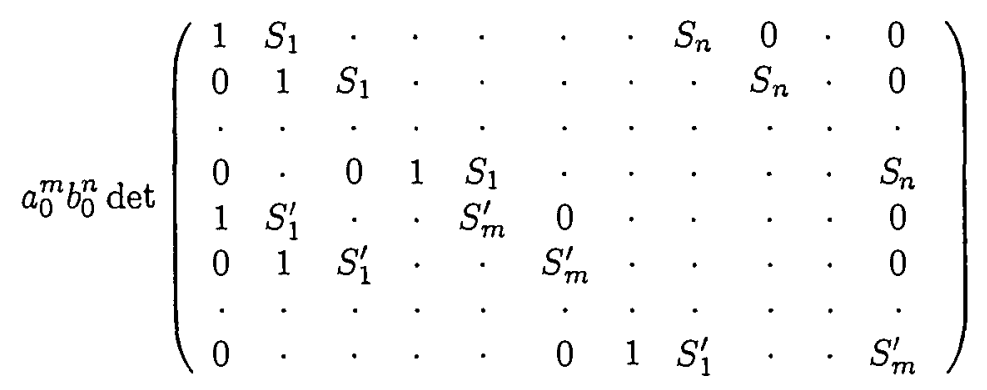

cujo determinante é $R_{Z}(F, G)$.

Das expressões de $F$ e $G$ segue que

$$
P=a_{0}^{m} \prod_{i=1}^{n} G\left(X_{i}\right)=(-1)^{n m} b_{0}^{n} \prod_{j=1}^{m} F\left(Y_{j}\right) .
$$

O termo de menor grau em $P$ que contém somente as indeterminadas de $\mathrm{Y}$ é $a_{0}^{m} b_{0}^{n}\left(Y_{1} \ldots Y_{m}\right)^{n}=$ $a_{0}^{m} b_{0}^{n} S_{m}^{\prime n}$.

Segue que

$$
R_{Z}(F, G)=a_{0}^{m} b_{0}^{n} \prod_{i=1}^{n} \prod_{j=1}^{m}\left(X_{i}-Y_{j}\right)=a_{0}^{m} \prod_{i=1}^{n} G\left(X_{i}\right)=(-1)^{n m} b_{0}^{n} \prod_{j=1}^{m} F\left(Y_{j}\right) .
$$

Substituindo as indeterminadas $X_{i}$ por $\alpha_{i}$ e $Y_{j}$ por $\beta_{j}$ o resultado segue. 
Definição 2.4.8 Seja $P \in \mathcal{R}[Y]$ e denotemos por $P_{Y}$ a derivada de $P$ em relação à $Y$. Definimos o discriminante de $P$ como sendo a resultante de $P$ e $P_{Y}$

$$
D_{Y}(P)=R_{Y}\left(P, P_{Y}\right)
$$

Se $P \in \mathcal{R}[Y]$ é um polinômio de Weierstrass, cuja decomposição em fatores irredutíveis em $\mathcal{R}[[Y]$ é dada por $P=P_{1}^{n_{1}} \ldots P_{r}^{n_{r}}$ e se $n_{i}>1$, para algum $i$, então $P$ e $P_{Y}$ tem fator comum não constante. Logo, segue do Corolário 2.4.3 que $D_{Y}(P)=0$. Portanto $D_{Y}(P) \neq 0$ se, e somente se, $n_{1}=n_{2}=$ $\ldots=n_{r}=1$.

Quando $n_{1}=\ldots=n_{r}=1$ dizemos que $P$ é reduzido. Se $P=P_{1}^{n_{1}} \ldots P_{r}^{n_{r}}$, definimos a redução de $P$ por

$$
\text { red } P=P_{1} \ldots P_{r}
$$

Proposição 2.4.9 Seja $f=a_{0} Y^{n}+a_{1} Y^{n-1}+\ldots+a_{n} \in k[Y] \backslash k$, onde $k$ é um corpo que contém as raizes $\alpha_{1}, \ldots, \alpha_{n}$ de $f$. Temos que

$$
D_{Y}(f)=a_{0}^{2 n-1} \prod_{i \neq j}\left(\alpha_{i}-\alpha_{j}\right)
$$

\section{Demonstração:}

Temos que $f=a_{0}\left(Y-\alpha_{1}\right) \ldots\left(Y-\alpha_{n}\right)$ em $k[Y]$. Logo,

$$
f_{Y}=\sum_{i=1}^{n} a_{0}\left(Y-\alpha_{1}\right) \ldots\left(\widehat{Y-\alpha_{i}}\right) \ldots\left(Y-\alpha_{n}\right)
$$

é um polinômio de grau $n-1$ em $k[Y] \mathrm{e}$

$$
f_{Y}\left(\alpha_{i}\right)=a_{0} \prod_{i \neq j}\left(\alpha_{i}-\alpha_{j}\right) .
$$

O resultado segue agora da Proposição 2.4.7:

$$
D_{Y}(f)=R_{Y}\left(f, f_{Y}\right)=a_{0}^{n-1} \prod_{i=1}^{n} f_{Y}\left(\alpha_{i}\right)=a_{0}^{n-1} \prod_{i=1}^{n} a_{0} \prod_{i \neq j}\left(\alpha_{i}-\alpha_{j}\right)=a_{0}^{2 n-1} \prod_{i \neq j}\left(\alpha_{i}-\alpha_{j}\right) .
$$




\section{Capítulo 3}

\section{Curvas Planas}

Neste capítulo vamos definir curvas irredutiveis algebróides planas ou, simplesmente, ramos planos. Estas curvas serão nosso principal objeto de estudo. Também vamos obter uma parametrização de Newton-Puiseux de um ramo plano sobre um corpo de característica zero.

\subsection{Curvas algebróides planas}

O estudo das singularidades de uma curva algébrica plana ou de uma curva analítica em $\mathbb{C}^{2}$, dada localmente pela equação

$$
f(X, Y)=0
$$

onde $f$ é um polinômio ou uma função analítica numa vizinhança da origem, pode ser realizado estudando as propriedades algébricas de $f(X, Y)$ como um elemento de $k[[X, Y]]$ e esta é a motivação para a seguinte definição.

Definiçāo 3.1.1 Uma curva algebróide plana $(f)$ é a classe de equivalência de uma elemento nâo invertível $f$ de $k[[X, Y]] \backslash\{0\}$, módulo a relação de associados.

Isto implica que $(f)=\{u f ; u$ é uma unidade em $k[[X, Y]]\}$.

Por definição, temos que $(f)=(g)$ se, e somente se, existe uma unidade $u \in k[[X, Y]]$ tal que $g=u f$.

Como a multiplicidade de uma série de potências é invariante por multiplicação por unidade, podemos definir a multiplicidade de uma curva algebróide plana $(f)$ como sendo a multiplicidade de $f$. Uma curva algebróide plana de multiplicidade 1 será chamada regular. Quando a multiplicidade for maior que 1, diremos que a curva é singular.

Seja $(f)$ uma curva algebróide plana. Dizemos que a curva $(f)$ é irredutível se a série de potências $f$ é irredutível em $k[[X, Y]]$. A noção de irredutibilidade independe do representante $f$ de $(f)$, pois se $g$ é outro representante de $(f)$, então $g=f u, \operatorname{com} f$ irredutível e $u$ uma unidade, logo $g$ é irredutível. Uma curva algebróide plana irredutível será chamada de ramo plano.

Seja $(f)$ uma curva algebróide plana e consideremos a decomposição de $f$ em fatores irredutiveis em $k[[X, Y]], f=f_{1} f_{2} \ldots f_{r}$. As curvas algebróides planas $\left(f_{j}\right), j=1, \ldots, r$, são chamadas de ramos da curva $(f)$. A curva $(f)$ será chamada reduzida se $\left(f_{i}\right) \neq\left(f_{j}\right), \forall i \neq j$, isto é, quando $f_{i}$ e $f_{j}$ não são associados se $i \neq j$. 
Vamos agora definir uma relação de equivalência entre curvas algebróides planas e mostraremos que algumas propriedades destas curvas são invariantes módulo essa relação de equivalência.

Definiçāo 3.1.2 Duas curvas algebróides planas $(f)$ e $(g)$ são ditas equivalentes, e escrevemos $(f) \sim$ $(g)$, se existe um $k$-automorfismo $\phi$ de $k[[X, Y]]$ tal que

$$
\phi(f)=u g
$$

onde $u \in k[[X, Y]]$ é uma unidade.

Se $k$ é um corpo infinito, então toda curva é equivalente a uma curva definida por um polinômio de Weierstrass. De fato, dado $f \in k[[X, Y]] \backslash\{0\}$, com mult $(f)=n$, então, do Corolário 2.1.8 existem um automorfismo linear $T$ de $k[[X, Y]]$, uma unidade $u \in k[[X, Y]]$ e $A_{1}, \ldots, A_{n} \in k[[X]]$, com mult $\left(A_{i}\right) \geq i, i=1, \ldots, n$, tais que

$$
T(f) u=Y^{n}+A_{1} Y^{n-1}+\ldots+A_{n}:=P .
$$

Portanto, $(T(f))=(P)$, onde $P$ é um polinômio de Weierstrass.

Proposição 3.1.3 Se $(f)$ e $(g)$ são curvas regulares então $(f) \sim(g)$.

\section{Demonstração:}

Como mult $(f)=1$, segue que

$$
f=(a X+b Y)+f_{2}+f_{3}+\ldots
$$

com $a \neq 0$ ou $b \neq 0$.

Se $a \neq 0$, podemos considerar $\circ k$-automorfismo $\phi=\mathcal{S}_{f, Y}$ definido por

$$
\begin{aligned}
\phi: k[[X, Y]] & \longrightarrow k[[X, Y]] \\
X & \longmapsto \\
Y & \longmapsto
\end{aligned}
$$

Logo, $(X) \sim(f)$ :

Se $b \neq 0$, podemos considerar o $k$-automorfismo $\dot{\psi}=\mathcal{S}_{X, f}$ definido por

$$
\begin{aligned}
\psi: k[[X, Y]] & \longrightarrow k[[X, Y]] \\
X & \longrightarrow \\
Y & \longrightarrow X
\end{aligned}
$$

Logo, $(Y) \sim(f)$.

Analogamente, $(X) \sim(g)$ ou $(Y) \sim(g)$. Como $(X) \sim(Y)$, segue que $(f) \sim(g)$.

Um problema na teoria de curvas alġebróides planas é a classificação das curvas segundo esta relação de equivalência. No entanto, ainda não existe um algoritmo que nos permita decidir quando duas curvas são equivalentes. 
Por exemplo, sejam

$$
f=Y^{2}-X^{5}
$$

e

$g=-3125 Y^{5}+(6250 X) Y^{4}+\left(-5000 X^{2}\right) Y^{3}+\left(2000 X^{3}+X^{2}\right) Y^{2}+\left(-400 X^{4}+2 X^{2}\right) Y-32 X^{5}+X^{2}$.

Essas curvas são equivalentes? Observemos que mult $(f)=$ mult $(g)=2$.

Neste caso, a série $g$ foi escolhida de modo que $\phi(f)=g$, onde

$$
\phi(X, Y)=(2 X-5 Y, X+X Y)
$$

Portanto, $(f) \sim(g)$.

Seja $(f)$ uma curva algebróide plana de multiplicidade $n, f=F_{n}+F_{n+1}+\ldots$, ondé cada $F_{i}$ é homogêneo de grau $i$ e $F_{n} \neq 0$. Chamamos a curva $\left(F_{n}\right)$ de cone tangente da curva $(f)$. Como qualquer polinômio homogêneo em 2 indeterminadas em um corpo algebricamente fechado decompõese em fatores lineares, (ver [10]), podemos escrever

$$
F_{n}=\prod_{i=1}^{s}\left(a_{i} X+b_{i} Y\right)^{r_{i}},
$$

onde $\sum_{i=1}^{s} r_{i}=n, a_{i}, b_{i} \in k, \forall i, j=1, \ldots, s$ e $a_{i} b_{j}-a_{j} b_{i} \neq 0$ se $i \neq j$. Então o cone tangente de $(f)$ consiste das formas lineares $\left(a_{i} X+b_{i} Y\right)$, onde $r_{i}$ é a multiplicidade da reta $\left(a_{i} X+b_{i} Y\right), i=1, \ldots, s$. Tais formas lineares são chamadas de retas tangentes de $(f)$.

Se a curva $(f)$ tem multiplicidade 1 , isto é, $(f)$ é regular, então seu cone tangente $\left(F_{1}\right)$ consiste de uma reta tangente com multiplicidade 1.

Exemplo 3.1.4 O cone tangente da curva $\left(Y^{2}-X^{5}\right)$ é $\left(Y^{2}\right)$ e sua reta tangente é $(Y)$ com multiplicidade 2 , enquanto que o cone tangente à curva $\left(Y^{2}-X^{2}(X+1)\right)$ é dado por $\left(Y^{2}-X^{2}\right)$ e consiste das retas tangentes $(Y+X)$ e $(Y-X)$, cada uma destas com multiplicidade 1 . A curva $\left(Y-X^{2}\right)$ é regular e sua reta tangente é $(Y)$.

\subsection{O Teorema de Newton-Puiseux}

No que segue $k$ é um corpo algebricamente fechado e de característica zero, logo $k$ é infinito. Vimos na seção anterior que neste caso toda curva algebróide plana é equivalente a ựa curva definida por um polinômio de Weierstrass em $k[[X]][Y]$. Utilizaremos este fato para estudar as curvas algebróides irredutíveis planas.

Também nesta seção vamos caracterizar o fecho algébrico $\overline{k((X))}$ de $k((X))$ e, para isto, vamos precisar de algumas definições básicas da Teoria de Corpos. Dizemos que um corpo $L$ é algebricamente fechado se todo polinômio em $L[X]$ de grau $\geq 1$ tem uma raiz em $L$. Dado $k$ um corpo qualquer, existe um corpo algebricamente fechado $L$ que contém $k$ como subcorpo (ver [3], Teorema 2.5, pag. 231). Este corpo $L$ é chamado de fecho algébrico de $k$ e será denotado por $\bar{k}$.

Na seção 1.3 vimos que $k((X))$ é o corpo das séries de Laurent com coeficientes em $k$, isto é, as séries da forma

$$
b_{-m} X^{-m}+b_{-m+1} X^{-m+1}+\ldots+b_{-1} X^{-1}+b_{0}+b_{1} X+\ldots
$$


onde $m \in \mathbb{N}$ e $b_{i} \in k, \forall i$.

Temos que $\overline{k((X))}$ deve conter as raízes dos polinômios $Y^{n}-X=0$, para todo inteiro positivo $n$, e portanto deve conter os elementos da forma $X^{\frac{1}{n}}$, sujeitos às seguintes relaçōes:

(i) $X^{\frac{1}{1}}=X$.

(ii) $\left(X^{\frac{m}{r n}}\right)^{r}=X^{\frac{m}{n}}, \forall m, n, r \in \mathbb{N} ; n, r>0$.

Definição 3.2.1 Seja $F / k$ uma extensão de corpos $(k \subset \mathcal{F})$. Se o grupo

$$
G(F / k)=\{\sigma: F \longrightarrow F ; \sigma \text { é um k-automorfismo }\}
$$

é finito e seu corpo fixo é $k$, ou seja,

$$
\{a \in F ; \sigma(a)=a, \forall \sigma \in G(F / k)\}=k
$$

então a extensão é chamada finita e galoisiana e $G(F / k)$ é seu grupo de Galois.

Denotaremos por $U_{n}$ o grupo multiplicativo das $n$-ésimas raízes da unidade em $k$. Temos que $U_{n}$ é um grupo cíclico de ordem $n$ (ver [3], pag. 277), ou seja, existe um elemento $u \in U_{n}$, tal que $U_{n}=\left\{1, u, u^{2}, \ldots, u^{n-1}\right\}$. O elemento $u$ é um gerador de $U_{n}$ e é chamado uma raiz $n$-ésima primitiva da unidade.

Seja $k\left(\left(X^{\frac{1}{n}}\right)\right)$ o corpo das séries de potências da forma

$$
b_{-m} X^{\frac{-m}{n}}+b_{-m+1} X^{\frac{-m+1}{n}}+\ldots+b_{-1} X^{\frac{-1}{n}}+b_{0}+b_{1} X^{\frac{1}{n}}+b_{2} X^{\frac{2}{n}} \ldots
$$

onde $m \in \mathbb{N}$ e $b_{i} \in k, \forall i$. Então $k\left(\left(X^{\frac{1}{n}}\right)\right)$ é $k$-isomorfo a $k((X))$ e, pela Proposição 1.3.1, temos que qualquer $k$-automorfismo $\sigma$ de $k\left(\left(X^{\frac{1}{n}}\right)\right)$ é tal que $\sigma\left(X^{\frac{1}{n}}\right)=X^{\frac{1}{n}} u\left(X^{\frac{1}{n}}\right)$, para alguma unidade $u \in k\left[\left[X^{\frac{1}{n}}\right]\right]$.

Lema 3.2.2 A extensão $k\left(\left(X^{\frac{1}{n}}\right)\right) / k((X))$ é finita e galoisiana com grupo de Galois isomorfo ao grupo $U_{n}$.

\section{Demonstração:}

Sejam $G=G\left(k\left(\left(X^{\frac{1}{n}}\right)\right) / k((X))\right)$ e $\sigma \in G$. Como $\sigma$ é um $k((X))$-automorfismo, segue da observação acima que existe uma unidade $b_{\sigma} \in k\left[\left[X^{\frac{1}{n}}\right]\right]$ tal que

$$
\sigma\left(X^{\frac{1}{n}}\right)=X^{\frac{1}{n}} b_{\sigma}\left(X^{\frac{1}{n}}\right)
$$

Como $\sigma \in G$ e o corpo fixo de $G$ contém $k((X))$, segue que

$$
X=\sigma(X)=\left(\sigma\left(X^{\frac{1}{n}}\right)\right)^{n}=\left(X^{\frac{1}{n}} b_{\sigma}\left(X^{\frac{1}{n}}\right)\right)^{n}=X\left(b_{\sigma}\left(X^{\frac{1}{n}}\right)\right)^{n} .
$$

Portanto, $\left(b_{\sigma}\left(X^{\frac{1}{n}}\right)\right)^{n}=1$ e $b_{\sigma} \in U_{n}$.

A aplicação $h: G \longrightarrow U_{n}$, definida por $h(\sigma)=b_{\sigma}$, é um isomorfismo de grupos. De fato, se $\rho \in G$ então

$$
. b_{\rho \circ \sigma} X^{\frac{1}{n}}=\rho \circ \sigma\left(X^{\frac{1}{n}}\right)=\rho\left(b_{\sigma} X^{\frac{1}{n}}\right)=b_{\sigma} \rho\left(X^{\frac{1}{n}}\right)=b_{\rho} b_{\sigma} X^{\frac{1}{n}} .
$$


Logo, $h(\rho \circ \sigma)=b_{\rho \circ \sigma}=b_{\rho} b_{\sigma}=h(\rho) h(\sigma)$ e, portanto, $h$ é um homomorfismo de.grupos. Suponhamos agora que $b_{\rho}=b_{\sigma}$. Como

$$
\sigma\left(\sum b_{i} X^{\frac{i}{n}}\right)=\sum b_{i}\left(\sigma\left(X^{\frac{1}{n}}\right)\right)^{i}=\sum b_{i} b_{\sigma}^{i} X^{\frac{i}{n}}=\sum b_{i} b_{\rho}^{i} X^{\frac{i}{n}}=\rho\left(\sum b_{i} X^{\frac{i}{n}}\right) .
$$

Isto mostra que $\sigma=\rho$ e, portanto, $h$ é injetora. Segue da definição de $\sigma$ que $h$ é sobrejetora. Portanto $G$ é isomorfo à $U_{n}$.

Mostremos agora que o corpo fixo de $G$ é precisamente $k((X))$. Já sabemos que o corpo fixo de $G$ contém $k((X))$. Suponhamos que $\sum_{i \geq i_{0}} b_{i} X^{\frac{i}{n}} \in k\left(\left(X^{\frac{1}{n}}\right)\right)$ é invariante pela ação dé elementos de $G$, isto é, $\forall \xi \in U_{n}$,

$$
\sum_{i \geq i_{0}} b_{i} X^{\frac{i}{n}}=\sigma\left(\sum_{i \geq i_{0}} b_{i} X^{\frac{i}{n}}\right)=\sum_{i \geq i_{0}} b_{i} \xi^{i} X^{\frac{i}{n}} .
$$

Logo, $b_{i}=b_{i} \xi^{i}, \forall i \geq i_{0}$. Se $\xi$ é uma raiz $n$-ésima primitiva da unidade e $i$ não divisível por $n$, então $\xi^{i} \neq 1$ e como $b_{i}\left(1-\xi^{i}\right)=0$, segue que $b_{i}=0$. Portanto, $\sum b_{i} X^{\frac{i}{n}} \in k((X))$, o que conclui a prova.

Do lema acima temos que toda extensão do tipo $k\left(\left(X^{\frac{1}{n}}\right)\right)$, com $n$ inteiro positivo, é finita sobre $k((X))$. Como toda extensão finita é algébrica (ver [3], Proposição 1.1, pag. 224), segue que todo elemento $\alpha \in k\left(\left(X^{\frac{1}{n}}\right)\right)$ é algébrico sobre $k((X))$. Mas, o fecho algébrico de um corpo contém todos os elementos que são algébricos sobre ele. Portanto, os corpos do tipo $k\left(\left(X^{\frac{1}{n}}\right)\right)$ èstão contidos em $\overline{k((X))}$. Podemos então definir

$$
k((X))^{*}=\bigcup_{n=1}^{\infty} k\left(\left(X^{\frac{1}{n}}\right)\right) \subset \overline{k((X))} .
$$

Os elementos de $k((X))^{*}$ podem ser escritos na forma

$$
\alpha=b_{1} X^{\frac{p_{1}}{q_{1}}}+b_{2} X^{\frac{p_{2}}{q_{2}}}+\ldots
$$

$\operatorname{com} b_{i} \in k, p_{i}, q_{i} \in \mathbb{Z}, q_{i}>0, \forall i, \frac{p_{1}}{q_{1}}<\frac{p_{2}}{q_{2}}<\ldots$, e o conjunto $\left\{\frac{p_{i}}{q_{i}} ; i \in \mathbb{N} \backslash\{0\}\right\}$ admite um deriominador comum.

Se $b_{1} \neq 0$, então o número racional $\frac{p_{1}}{q_{1}}$ é chamado de multiplicidade de $\alpha$ e será denotado por mult $(\alpha)$. Definimos mult $(0)=\infty$.

Dados $\alpha, \beta \in k((X))^{*}$, temos que

$$
\begin{gathered}
\text { mult }(\alpha \cdot \beta)=\operatorname{mult}(\alpha)+\operatorname{mult}(\beta) \\
\text { mult }(\alpha \pm \beta) \geq \min \{\operatorname{mult}(\alpha), \operatorname{mult}(\beta)\}
\end{gathered}
$$

valendo a igualdade sempre que mult $(\alpha) \neq \operatorname{mult}(\beta)$.

Também definimos

$$
k[[X]]^{*}=\bigcup_{i=1}^{\infty} k\left[\left[X^{\frac{1}{n}}\right]\right] .
$$

Portanto, todo elemento $\alpha$ de $k[[X]]^{*}$ é da forma

$$
\alpha=b_{1} X^{\frac{p_{1}}{q_{1}}}+b_{2} X^{\frac{p_{2}}{q_{2}}}+\ldots
$$

onde $b_{i} \in k, p_{i}, q_{i} \in \mathbb{N}, q_{i}>0, \forall i, \frac{p_{1}}{q_{1}}<\frac{p_{2}}{q_{2}}<\ldots$, e o conjunto $\left\{\frac{p_{i}}{q_{i}} ; i \in \mathbb{N} \backslash\{0\}\right\}$ admite um denominador comum. Assim, todo elemento de $k[[X]]^{*}$ tem multiplicidade maior ou igual a zero. 
Lema 3.2.3 $k((X))^{*}$ é um subcorpo de $\overline{k((X))}$.

\section{Demonstração:}

Se $f, g \in k((X))^{*}$, existem $r, s \in \mathbb{N}$ tais que $f \in k\left(\left(X^{\frac{1}{r}}\right)\right)$ e $g \in k\left(\left(X^{\frac{1}{s}}\right)\right)$. Como $k\left(\left(X^{\frac{1}{r}}\right)\right) \subset k\left(\left(X^{\frac{1}{r s}}\right)\right)$ e $k\left(\left(X^{\frac{1}{s}}\right)\right) \subset k\left(\left(X^{\frac{1}{r s}}\right)\right)$, temos que $f+g, f \cdot g$ e $\frac{f}{g}$, se $g \neq 0$, estão em $k\left(\left(X^{\frac{1}{r s}}\right)\right) \subset k((X))^{*}$

O próximo resultado desempenha um papel central na Teoria de Curvas Algebróides Irredutíveis Planas definidas em corpos algebricamente fechados e de característica zero, pois permite obter uma parametrização para um ramo plano.

Teorema 3.2.4 (Newton-Puiseux) Temos que $\overline{k((X))}=k((X))^{*}$.

\section{Demonstração:}

Vimos acima que $k((X)) \subset k((X))^{*} \subset \overline{k((X))}$. Logo, $\overline{k((X))} \subset \overline{k((X))^{*}}$. Portanto, é suficiente mostrar que $k((X))^{*}$ é algebricamente fechado. Para tanto, basta mostrar que todo polinômio em $k((X))^{*}[Y]$ de grau $\geq 2$ é redutível em $k((X))^{*}[Y]$, pois todo polinômio de grau 1 tem raiz.

Seja

$$
p(X, Y)=a_{0}(X) Y^{n}+a_{1}(X) Y^{n-1}+\ldots+a_{n}(X) \in k((X))^{*}[Y],
$$

com $n \geq 2$ e $a_{0}(X) \neq 0$. Vamos supor, sem perda de generalidade, que $a_{0}(X)=1$.

Vamos eliminar o termo de grau $n-1 \mathrm{em} p(X, Y)$ através de uma mudança de variáveis. Consideremos o $k((X))^{*}$-isomorfismo

$$
\begin{array}{ccc}
\phi: k((X))^{*}[Y] & \longrightarrow & k((X))^{*}[Z] \\
Y & \longmapsto & Z-n^{-1} a_{1}(X)
\end{array}
$$

Assim,

$$
\begin{gathered}
q(X, Z):=\phi(p(X, Y))=p\left(X, Z-n^{-1} a_{1}(X)\right)= \\
=\left(Z-n^{-1} a_{1}(X)\right)^{n}+a_{1}(X)\left(Z-n^{-1} a_{1}(X)\right)^{n-1}+\ldots+a_{n}(X) \\
=Z^{n}+b_{2}(X) Z^{n-2}+\ldots+b_{n}(X),
\end{gathered}
$$

onde $b_{i}(X) \in k((X))^{*}, i=2, \ldots, n$.

Se $b_{i}(X)=0, \forall i=2, \ldots, n$, então $q$ é redutível em $k((X))^{*}[Z]$ e portanto $p$ é redutível em $k((X))^{*}[Y]$.

Suponhamos agora que exista um índice $i$ tal que $b_{i}(X) \neq 0$. Denotaremos por $u_{i}$ a multiplicidade de $b_{i}(X)$ e

$$
u=\min \left\{\frac{u_{i}}{i}: 2 \leq i \leq n\right\}
$$

Seja $r$ tal que $u=\frac{u_{r}}{r}$ e consideremos a aplicação

$$
\begin{aligned}
\psi: k((X))^{*}[Z] & \longrightarrow k((W))^{*}[Z] \\
f(X, Z) & \longmapsto f\left(W^{r}, Z W^{u_{r}}\right)
\end{aligned}
$$

Temos que $\psi$ é um $k$-isomorfismo, onde $\psi^{-1}(g(W, Z))=g\left(X^{\frac{1}{r}}, X^{-u} Z\right)$. Além disso, $\psi$ preserva o grau como polinômio em $Z$. De fato, se $f(X, Z)=a_{0}(X) Z^{n}+a_{1}(X) Z^{n-1}+\ldots+a_{n}(X)$, com $a_{i}(X) \in k((X))^{*}, i=0, \ldots, n$, então

$$
\psi(f(X, Z))=f\left(W^{r}, Z W^{u_{r}}\right)=a_{0}\left(W^{r}\right)\left(Z W^{u_{r}}\right)^{n}+a_{1}\left(W^{r}\right)\left(Z W^{u_{r}}\right)^{n-1}+\ldots+a_{n}\left(W^{r}\right)=
$$




$$
a_{0}\left(W^{r}\right) W^{u_{r} n} Z^{n}+a_{1}\left(W^{r}\right) W^{u_{r}(n-1)} Z^{n-1}+\ldots+a_{n}\left(W^{r}\right) .
$$

Portanto, $\psi$ preserva o grau como polinômio em $Z$.

Seja

$$
\begin{gathered}
h(W, Z):=W^{-n u_{r}} \psi(q(X, Z))=W^{-n u_{r}} q\left(W^{r}, Z W^{u_{r}}\right)= \\
=W^{-n u_{r}}\left(Z^{n} W^{u_{r} n}+b_{2}\left(W^{r}\right) Z^{n-2} W^{u_{r}(n-2)}+\ldots+b_{n}\left(W^{r}\right)\right)=Z^{n}+\sum_{i=2}^{n} c_{i}(W) Z^{n-i},
\end{gathered}
$$

onde $c_{i}(W)=b_{i}\left(W^{r}\right) W^{-n u_{r}} W^{u_{r}(n-i)}=b_{i}\left(W^{r}\right) W^{-i u_{r}}$. Temos que

$$
\text { mult }\left(c_{i}\right)=u_{i} r-i u_{r} \geq 0
$$

pois $\frac{u_{i}}{i} \geq \frac{u_{r}}{r}, \forall i$, e mult $\left(c_{i}\right)=0$ se $i=r$. Logo, $c_{r}(0) \neq 0$ e $c_{i}(W) \in k[[W]]^{*}, \forall i=2, \ldots, n$.

Então existe um inteiro positivo $c$ tal que

$$
h\left(W^{c}, Z\right)=Z^{n}+\sum_{i=2}^{n} c_{i}\left(W^{c}\right) Z^{n-i} \in k[[W]][Z] .
$$

Como $c_{r}(0) \neq 0$ e car $k=0$ então $h(0, Z)$ tem pelo menos 2 raízes distintas. De faro, como $c_{r}(0) \neq 0, h(0, Z)$ tem uma raiz não nula. Se $h(0, Z)=(Z+a)^{n}$, comparando os termos de grau $n-1$ temos $0=n a$, donde $a=0$, o que é uma contradição. Pelo Lema de Hensel 1.4.2, existem $h_{1}$ e $h_{2} \mathrm{em}$ $k[[W]][Z]$ de graus $\geq 1$ em $Z$ tais que

$$
\dot{h}\left(W^{c}, Z\right)=h_{1}(W, Z) h_{2}(W, Z)
$$

Logo,

$$
\psi(q(X, Z))=W^{n u_{r}} h(W, Z)=W^{n u_{r}} h_{1}\left(W^{\frac{1}{c}}, Z\right) h_{2}\left(W^{\frac{1}{c}}, Z\right)
$$

Portanto,

$$
q(X, Z)=\psi^{-1}\left(W^{n u_{r}} h_{1}\left(W^{\frac{1}{c}}, Z\right) h_{2}\left(W^{\frac{1}{c}}, Z\right)\right) .
$$

Como $\psi^{-1}(W)=X^{\frac{1}{r}}$, temos que

$$
q(X, Z)=X^{\frac{n u_{r}}{r}} \psi^{-1}\left(h_{1}\left(W^{\frac{1}{c}}, Z\right)\right) \psi^{-1}\left(h_{2}\left(W^{\frac{1}{c}}, Z\right)\right) .
$$

Segue que $q(X, Z)$ é redutível em $k((X))^{*}[Z]$.

O teorema acima não é válido se car $k>0$.

\subsection{Extensões do corpo das séries de Laurent}

Vịmos que o grupo de Galois da extensão $k\left(\left(X^{\frac{1}{n}}\right)\right) / k((X))$ é isomorfo a $U_{n}$. Assim um elemento $\rho \in U_{n}$ age em um elemento

$$
\alpha=\sum_{i \geq i_{0}} b_{i}\left(X^{\frac{1}{n}}\right)^{i}=\sum_{i \geq i_{0}} b_{i} X^{\frac{i}{n}} \in k\left(\left(X^{\frac{1}{n}}\right)\right)
$$

da seguinte forma

$$
\rho * \alpha=\sum_{i \geq i_{0}} b_{i}\left(\rho X^{\frac{1}{n}}\right)^{i}=\sum_{i \geq i_{0}} b_{i} \rho^{i} X^{\frac{i}{n}}
$$


Lema 3.3.1 Sejam $\alpha \in k((X))^{*} \backslash k((X))$ e $n=\min \left\{q \in \mathbb{N} ; \alpha \in k\left(\left(X^{\frac{1}{q}}\right)\right)\right\}$. Considerando $\alpha$ como um elemento de $k\left(\left(X^{\frac{1}{n}}\right)\right)$, temos que $\xi * \alpha \neq \rho * \alpha, \forall \xi, \rho \in U_{n}$, com $\xi \neq \rho$.

\section{Demonstração:}

Como $\alpha \notin k((X))$, então $n \geq 2$. Escrevendo

$$
\alpha=\varphi\left(X^{\frac{1}{n}}\right)=\sum_{i \geq i_{0}} b_{i} X^{\frac{i}{n}}
$$

vamos supor, por contradição, que $\varphi\left(\rho X^{\frac{1}{n}}\right)=\varphi\left(\xi X^{\frac{1}{n}}\right), \rho, \xi \in U_{n}, \rho \neq \xi$. Então $\rho^{i} b_{i}=\xi^{i} b_{i}$ e, portanto, $\rho^{i}=\xi^{i}$ para todo $i$ tal que $b_{i} \neq 0$.

Seja $d$ o máximo divisor comum entre $n$ e os $i^{\prime}$ s para os quais $b_{i} \neq 0$. Como o conjunto $\left\{i \in \mathbb{N} ; b_{i} \neq 0\right\}$ pode ser infinito, $d$ é o ínfimo do conjunto dos máximos divisores comuns entre $n$ e um número finito desses $i$ 's. Afirmamos que $d=1$ pois, caso contrário, teríamos $\alpha \in k\left(\left(X^{\frac{1}{n^{\prime}}}\right)\right)$, onde $n^{\prime}=\frac{n}{d}<n$, o que é um absurdo, pois $n=\min \left\{q \in \mathbb{N} ; \alpha \in k\left(\left(X^{\frac{1}{q}}\right)\right)\right\}$.

Isto mostra que existem índices $i_{1}, \ldots, i_{k}$ tais que $b_{i_{j}} \neq 0, j=1, \ldots, k, \mathrm{e} v, v_{1}, \ldots, v_{k} \in \mathbb{Z}$, tais que

$$
v n+v_{1} i_{1}+\ldots+v_{k} i_{k}=1
$$

e, portanto,

$$
\xi=\left(\xi^{n}\right)^{v}\left(\xi^{i_{1}}\right)^{v_{1}} \ldots\left(\xi^{i_{k}}\right)^{v_{k}}=\left(\rho^{n}\right)^{v}\left(\rho^{i_{1}}\right)^{v_{1}} \ldots\left(\rho^{i_{k}}\right)^{v_{k}}=\rho
$$

o que contradiz a hipótese de que $\xi \neq \rho$.

No próximo resultado vamos descrever as principais extensões algébricas de $k((X))$, isto é, os corpos $k((X))(\alpha)$, obtidos adicionando à $k((X))$ o elemento algébrico $\alpha$. Lembramos que um elemento $\alpha$ é algébrico.sobre $k((X))$ se existem $\alpha_{0}(X), \alpha_{1}(X), \ldots, \alpha_{n}(X) \in k((X))$, não todos nulos, tais que se

$$
F(Y)=\alpha_{0}(X) Y^{n}+\alpha_{1}(X) Y^{n-1}+\ldots+\alpha_{n}(X) \in k((X))[Y]
$$

então $F(\alpha)=0$. Temos que $k((X))(\alpha)$ é o menor corpo que contém $k((X))$ e $\alpha$, e consiste de todos os quocientes $\frac{f(\alpha)}{g(\alpha)}$, "onde $f$ e $g$ são polinômios em $k((X))[Y]$ e $g(\alpha) \neq 0$. Como $\alpha$ é algébrico sobre $k((X))$, temos que

$$
k((X))(\alpha)=k((X))[\alpha]=\{p(\alpha) ; p \in k((X))[Y]\}
$$

(ver [3], Proposição 1.4, pag. 225).

Seja $F / k$ uma extensão de corpos. Vamos definir o grau dessa extensão como sendo a dimensão de $F$ como espaço vetorial sobre $k$ e denotaremos por $[F: k]$.

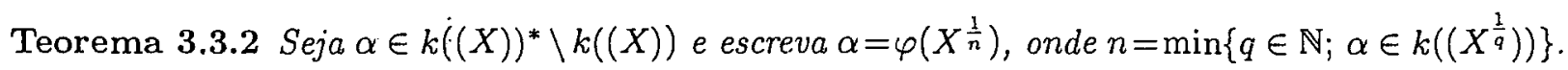
Então

(i) $k((X))[\alpha]=k\left(\left(\dot{X}^{\frac{1}{n}}\right)\right)$

(ii) O polinômio minimal de $\alpha$ sobre $k((X))$ é dado por

$$
g(X, Y)=\prod_{i=1}^{n}\left(Y-\alpha_{i}\right)
$$

onde $\alpha_{i}=\varphi\left(\zeta^{i} X^{\frac{1}{n}}\right)$ e $\zeta \in U_{n}$ é uma raiz $n$-ésima primitiva da unidade. 
(iii) Temos que $g(X, Y)=Y^{n}+a_{1}(X) Y^{n-1}+\ldots+a_{n}(X) \in k((X))[Y]$, onde

$$
\text { mult }\left(a_{i}(X)\right) \geq i \text { mult }(\alpha)=i \frac{\operatorname{mult}\left(a_{n}(X)\right)}{n}
$$

e a igualdade ocorre quando $i=n$. Em particular, se mult $(\alpha) \geq 1$ (resp. mult $(\alpha)>0$ ) então $g(X, Y) \in k[[X]][Y]$ é um polinômio de Weierstrass (resp. um pseudo-polinômio).

\section{Demonstração:}

(i) Sejam $G=G\left(k\left(\left(X^{\frac{1}{n}}\right)\right) / k((X))\right) \mathrm{e}$ $G^{\prime}=G\left(k\left(\left(X^{\frac{1}{n}}\right)\right) / k((X))[\alpha]\right)=\left\{\sigma: k\left(\left(X^{\frac{1}{n}}\right)\right) \longrightarrow k\left(\left(X^{\frac{1}{n}}\right)\right) ; \sigma\right.$ é um $k((X))[\alpha]$ - automorfismo $\}$

Observemos que $G^{\prime} \subset G$ e que $G^{\prime}=\{\sigma \in G ; \sigma * \alpha=\alpha\}$ Mas, se

$$
\sigma * \alpha=\sum_{i \geq i_{0}} b_{i}\left(\sigma X^{\frac{1}{n}}\right)^{i}=\sum_{i \geq i_{0}} b_{i} \sigma^{i} X^{\frac{i}{n}}=\sum_{i \geq i_{0}} b_{i} X^{\frac{i}{n}}=\alpha
$$

então $\sigma^{i}=1$, para todo $i \geq i_{0}$. Segue que $\sigma$ é a identidade e, portanto, $k\left(\left(X^{\frac{1}{n}}\right)\right)=k((X))[\alpha]$.

(ii) Como $\alpha_{i}=\varphi\left(\zeta^{i} X^{\frac{1}{n}}\right), i=1, \ldots, n$, são as transformações de $\alpha$ pelos elementos de $G$, temos que $g(X, Y) \in k((X))[Y]$.

Também temos que,

$$
\partial(g(X, Y))=[k((X))[\alpha]: k((X))]=\left[k\left(\left(X^{\frac{1}{n}}\right)\right): k((X))\right]=n
$$

pois o grupo de Galois da extensão $k\left(\left(X^{\frac{1}{n}}\right)\right) / k((X))$ tem $n$ elementos (ver [3]; Teorema 1.8, pag. 264). Como $\alpha$ é algébrico sobre $k((X))$ e $[k((X))[\alpha]: k((X))]=n$, temos que $n$ é o' grau do polinômio irredutível de menor grau em $k((X))[Y]$ satisfeito por $\alpha$. Portanto, $g(X, Y)$ é irredutível em $k((X))[Y]$. Logo, $g(X, Y)$ é o polinômio minimal de $\alpha$ sobre $k((X))$.

(iii) Se $S_{i}, i=1, \ldots, n$, são as funçōes simétricas elementares em $\alpha_{1}, \ldots, \alpha_{n}$, então os coeficientes de $g(X, Y)$, como polinômio em $Y$, são dados por

$$
a_{i}(X)=(-1)^{i} S_{i}\left(\alpha_{1}, \ldots, \alpha_{n}\right) \in k((X)) .
$$

Temos que mult $\left(\alpha_{j}\right)=\operatorname{mult}(\alpha), \forall j=1, \ldots, n$. Então

$$
\text { mult }\left(a_{i}(X)\right)=\operatorname{mult}\left((-1)^{i} S_{i}\left(\alpha_{1}, \ldots, \alpha_{n}\right)\right) \geq i \operatorname{mult}(\alpha) \geq i \frac{\operatorname{mult}\left(a_{n}(X)\right)}{n} .
$$

Se $i=n$, temos que mult $\left(a_{n}(X)\right)=n$ mult $(\alpha)$.

Se mult $(\alpha) \geq 1$ então mult $\left(a_{i}(X)\right) \geq i$ mult $(\alpha) \geq i$. Logo, $g(X, Y)$ é um polinômio de Weierstrass.

Se mult $(\alpha)>0$ então mult $\left(a_{i}(X)\right)>0$. Logo, $g(X, Y)$ é um pseudo-polinômio.

Corolário 3.3.3 Toda extensão finita de $k((X))$ é da forma $k\left(\left(X^{\frac{1}{n}}\right)\right)$ para algum $n \in \mathbb{N} \backslash\{0\}$. 


\section{Demonstração:}

Seja $k((X))\left(a_{1}, \ldots, a_{n}\right)$ uma extensão finita de $k((X))$. Como car $k((X))=0$ e os elementos $a_{1}, \ldots, a_{n}$ são algébricos sobre $k((X))$, temos que existe $a \in k((X))\left(a_{1}, \ldots, a_{n}\right)$ tal que

$$
k((X))\left(a_{1}, \ldots, a_{n}\right)=k((X))(a)
$$

(ver [5], Corolário, pag. 235). Mas, $k((X))(a)=k\left(\left(X^{\frac{1}{n}}\right)\right)$; para algum $n \in \mathbb{N} \backslash\{0\}$, pelo Teorema 3.3 .2 e o resultado segue.

Corolário 3.3.4 Seja $f \in k((X))[Y]$ um polinômio mônico irredutivel de grau $n \geq 1$, e seja $\alpha \in$ $k((X))^{*}$ uma raiz de $f$. Então,

(i) $\min \left\{q \in \mathbb{N} ; \alpha \in k\left(\left(X^{\frac{1}{a}}\right)\right)\right\}=n$.

(ii) Se $\alpha=\varphi\left(X^{\frac{1}{n}}\right)$ e $\alpha_{i}=\varphi\left(\zeta^{i} X^{\frac{1}{n}}\right)$, onde $\zeta \in U_{n}$ é uma raiz n-ésima primitiva da unidade, temos que

$$
f(X, Y)=\prod_{i=1}^{n}\left(Y-\alpha_{i}\right) .
$$

(iii) Se $f \in k[[X]][Y]$ é um polinômio de Weierstrass (resp. um pseudo-polinômio), então mult $(\alpha) \geq$ 1 (resp. mult $(\alpha)>0)^{\prime}$. Em particular, $\alpha \in k[[X]]^{*}$.

\section{Demonstração:}

(i) Temos que $\alpha$ é um elemento algébrico sobre $k((X))$, pois $f(\alpha)=0$, onde $f \in k((X))[Y]$ é um polinômio mônico irredutível de grau $n \geq 1$. Então $[k((X))[\alpha]: k((X))]=n$, e, portanto, $k((X))[\alpha]=k\left(\left(X^{\frac{1}{n}}\right)\right)$. Logo, $\min \left\{q \in \mathbb{N} ; \alpha \in k\left(\left(X^{\frac{1}{q}}\right)\right)\right\}=n$.

(ii) Como $f$ é um polinômio mônico irredutível de grau $n$ e $\alpha$ é raiz de $f$ segue que $f$ é o polinômio minimal de $\alpha$ sobre $k((X))$. Portanto, segue do Teorema 3.3.2 que $f(X, Y)=\prod_{i=1}^{n}\left(Y-\alpha_{i}\right)$.

(iii) Se $f=Y^{n}+a_{1}(X) Y^{n-1}+\ldots+a_{n}(X) \in k[[X][[Y]$ é um polinômio de Weierstrass, então

$$
-\alpha^{n}=a_{n}(X)+a_{n-1}(X) \alpha+\ldots+a_{1}(X) \alpha^{n-1} .
$$

Para algum $i_{0}=1, \ldots, n$, temos que

$$
\begin{gathered}
n \text { mult }(\alpha) \geq \min \left\{\text { mult }\left(a_{i}(X)\right)+(n-i) \text { mult }(\alpha)\right\}= \\
\text { mult }\left(a_{i_{0}}(X)\right)+\left(n-i_{0}\right) \text { mult }(\alpha) \geq i_{0}+\left(n-i_{0}\right) \text { mult }(\alpha) .
\end{gathered}
$$

Portanto, $i_{0}$ mult $(\alpha) \geq i_{0}$. Logo, mult $(\alpha) \geq 1$.

Se $f=Y^{n}+a_{1}(X) Y^{n-1}+\ldots+a_{n}(X)$ é um pseudo-polinômio, então

$$
n \text { mult }(\alpha) \geq \text { mult }\left(a_{i_{0}}(X)\right)+\left(n-i_{0}\right) \text { mult }(\alpha)>0+\left(n-i_{0}\right) \text { mult }(\alpha) \text {. }
$$


Portanto, $i_{0}$ mult $(\alpha)>0$. Logo, mult $(\alpha)>0$.

Em particular, $\alpha \in k[[X]]^{*}$.

Corolário 3.3.5 (Teorema da Função Implícita de Newton) Seja $f \in k[[X, Y]]$ irredutível e de multiplicidade n. Suponhamos que $\frac{\partial^{n} f}{\partial Y^{n}}(0,0) \neq 0$. Então existe

$$
\varphi\left(X^{\frac{1}{n}}\right)=\sum_{i \geq i_{0}} b_{i} X^{\frac{2}{n}} \in k\left[\left[X^{\frac{1}{n}}\right]\right]
$$

tal que

$$
f\left(X, \varphi\left(X^{\frac{1}{n}}\right)\right)=0 .
$$

Além disso, qualquer $\alpha \in k\left[\left[X^{\frac{1}{n}}\right]\right]$ satisfazendo $f(X, \alpha)=0$ é tal que $\alpha=\varphi\left(\zeta X^{\frac{1}{n}}\right)$, para algum $\zeta \in U_{n}$.

\section{Demonstração:}

Como mult $(f)=n$ e sua $n$-ésima derivada parcial com respeito a $Y$ em $(0,0)$ não se anula, temos que $f$ é regular com respeito a $Y$.

Segue do Teorema da Preparação de Weierstrass que $f$ é associado a um pseudo-polinômio em $k[[X]][Y]$ de grau $n$. Segue do corolário anterior que se $\alpha \in k((X))^{*}$ é raiz de $f$, então $\alpha \in k[[X]]^{*}$. Mais ainda, $\alpha=\varphi\left(X^{\frac{1}{n}}\right) \in k\left[\left[X^{\frac{1}{n}}\right]\right]$. Também é consequência do corolário anterior que qualquer $\alpha \in k\left[\left[X^{\frac{1}{n}}\right]\right]$ satisfaz

$$
f(X, Y) u=\prod_{i=1}^{n}\left(Y-\alpha_{i}\right)
$$

para alguma unidade $u$. Logo, o resultado segue.

Como $k[[X]]$ é D.F.U., com corpo de frações $k((X))$, todo polinômio mônico irredutível em $k[[X]][Y]$ é irredutível em $k((X))[Y]$.

O resultado que segue é uma condição de irredutibilidade de uma série de potências e tem fundamental importância geométrica.

Lema 3.3.6 (Lema da Unitangente) Seja $f \in k[[X, Y]]$ com $f(0,0)=0$, irredutivel de multiplicidade $n$. Então a forma inicial de $f$ é da forma

$$
F_{n}=(a X+b Y)^{n},
$$

onde $a, b \in k$, não são simultaneamente nulos.

\section{Demonstração:}

Toda curva $f$ é equivalente a uma curva definida por um polinômio de Weierstrass, ou seja, existem $p \in k[[X]][Y]$ de grau $n$ em $Y, u \in k[[X, Y]]$ unidade, tais que $u p=f$. Como $f$ é irredutível, segue do Lema 2.2.3 que $p$ é irredutível em $k[[X]][Y]$. Assim, $p$ é irredutível em $k((X))[Y]$. Do Corolário 3.3.4, temos que

$$
p(X, Y) u=\prod_{k=1}^{n}\left(Y-\varphi\left(\zeta^{k} X^{\frac{1}{n}}\right)\right),
$$


onde $\zeta$ é uma raiz $n$-ésima primitiva da unidade e

$$
\varphi\left(X^{\frac{1}{n}}\right)=b_{r} X^{\frac{r}{n}}+b_{r+1} X^{\frac{r+1}{n}}+\ldots \in k\left(\left(X^{\frac{1}{n}}\right)\right)
$$

$\operatorname{com} b_{r} \neq 0$.

Como $p$ é um polinômio de Weierstrass, segue do Corolário 3.3 .4 que mult $\left(\varphi\left(X^{\frac{1}{n}}\right)\right) \geq 1, \operatorname{logo}$ $\frac{r}{n} \geq 1$, o que implica $r \geq n$.

A forma inicial de $p$ é a forma inicial do polinômio $q$ dado por

$$
q(X, Y)=\prod_{k=1}^{n}\left(Y-\zeta^{k r} b_{r} X^{\frac{r}{n}}\right)=Y^{n}-\left(b_{r} X^{\frac{r}{n}} \sum_{k=1}^{n} \zeta^{k r}\right) Y^{n-1}+\ldots+(-1)^{n} b_{r} X^{\tau}
$$

Se $r=n$, a forma inicial de $p$ é $\left(Y-b_{n} X\right)$, pois

$$
\prod_{k=1}^{n}\left(Y-\zeta^{k n} b_{n} X^{\frac{n}{n}}\right)=\prod_{k=1}^{n}\left(Y-b_{n} X\right)=\left(Y-b_{n} X\right)^{n} .
$$

Se $r>n$ então $n-i+\frac{i r}{n}>n, \forall i=1, \ldots, n$. Portanto, a forma inicial de $p$ é $Y^{n}$.

Como a forma inicial de $f$ é o produto de $u(0,0) \neq 0$ pela forma inicial de $p$, segue que

$$
F_{n}=(a X+b Y)^{n}
$$

onde $a, b \in k$, não são simultaneamente nulos.

Este Lema nos mostra que se $f$ é irredutível então o cone tangente de $(f)$ consiste apenas da reta $(a X+b Y)$ contada com multiplicidade.

Exemplo 3.3.7 O seguintes polinômios são redutíveis em $k[[X, Y]]$.

- A cúbica nodal: $Y^{2}-X^{2}(X-1)$. Cone tangente: $Y^{2}+X^{2}=(Y-i X)(Y+i X)$.

- Folium de Descartes: $3 X Y-\left(X^{3}+Y^{3}\right)$. Cone tangente: $3 X Y$.

- Trissetriz de MacLaurin : $X\left(X^{2}+Y^{2}\right)-\left(Y^{2}-3 X^{2}\right)$. Cone tangente: $3 X^{2}-Y^{2}=(\sqrt{3} X+$ $Y)(\sqrt{3} X-Y)$.

- Conchóide de Nicomedes: $(Y-1)^{2}\left(X^{2}+Y^{2}\right)-2 Y^{2}$. Cone tangente: $X^{2}-Y^{2}=(X-Y)(X+Y)$.

\subsection{Parametrização e expoentes característicos}

Nesta seção vamos introduzir o conceito de parametrização de ramos planos.

Seja $f=F_{n}+F_{n+1}+\ldots \in k[[X, Y]]$ uma série de potências irredutível de multiplicidade $n$. Pelo Lema da Unitangente temos que $F_{n}=(a X+b Y)^{n}$, onde $a, b \in k$ não são simultaneamente nulos. Então, $f$ é regular em $Y$ (quando $b \neq 0$ ) ou $f$ é regular em $X$ (quando $a \neq 0$ ).

Se $f$ é regular em $Y$, podemos escrever $f$ na forma

$$
f=a_{0}(X) Y^{n}+a_{1}(X) Y^{n-1}+\ldots+a_{n}(X)+Y^{n+1} h(X, Y)
$$

onde $a_{i}(X) \in k[[X]]$, mult $\left(a_{i}(X)\right) \geq i, \forall i=1, \ldots, n, a_{0}(X) \neq 0$ e $h(X, Y) \in k[[X, Y]]$. 
Lema 3.4.1 Seja $f \in k[[X, Y]]$ uma série de potências irredutivel de multiplicidade $n$ e regular em $Y$. Escrevendo $f$ na forma

$$
f=a_{0}(X) Y^{n}+a_{1}(X) Y^{n-1}+\ldots+a_{n}(X)+Y^{n+1} h(X ; Y) .
$$

Temos que

$$
\text { mult }\left(a_{i}(X)\right) \geq i \frac{\text { mult }\left(a_{n}(X)\right)}{n}, \forall i=0, \ldots, n
$$

\section{Demonstração:}

Como $f$ é regular em $Y$ de ordem $n$, segue do Teorema da Preparação de Weierśstrass que existem uma unidade $u \in k[[X, Y]]$ e $A_{1}, \ldots, A_{n} \in k\left[\left[X_{i}\right], A_{i}(0) \neq 0, i=1, \ldots, n\right.$, tais que

$$
u f=Y^{n}+A_{1} Y^{n-1}+\ldots+A_{n} .
$$

Como $f$ é irredutível, segue do Teorema 3.3.2(iii) que

$$
\text { mult }\left(A_{i}(X)\right) \geq i \frac{\operatorname{mult}\left(A_{n}(X)\right)}{n}, \forall i=1, \ldots, n \text {. }
$$

Escrevendo $u=u_{0}+u_{1} Y+\ldots$, com $u_{i} \in k[[X]]$ e $u_{0}(0) \neq 0$, temos que $\left(u_{0}+u_{1} Y+\ldots\right)\left(a_{0}(X) Y^{n}+a_{1}(X) Y^{n-1}+\ldots+a_{n}(X)+Y^{n+1} h(X, Y)\right)=Y^{n}+A_{1} Y^{n-1}+\ldots+A_{n}$. Portanto,

$$
u_{0} a_{i}+u_{1} a_{i+1}+\ldots+u_{n-i} a_{n}=A_{i}, \forall i=1, \ldots, n
$$

Em particular,

$$
\operatorname{mult}\left(a_{n}\right)=\operatorname{mult}\left(u_{0} a_{n}\right)=\operatorname{mult}\left(A_{n}\right)
$$

Temos que, $u_{0} a_{i}=A_{i}-u_{1} a_{i+1}-\ldots-u_{n-i} a_{n}$. Logo,

mult $\left(a_{i}\right)=\operatorname{mult}\left(u_{0} a_{i}\right)=\operatorname{mult}\left(A_{i}-\left(u_{1} a_{i+1}+\ldots+u_{n-i} a_{n}\right)\right) \geq \operatorname{mult}\left(A_{i}\right) \geq i \frac{\operatorname{mult}\left(a_{n}(X)\right)}{n}$.

Lema 3.4.2 Seja $f$ como no lema anterior. As seguintes condiçōes são equivalentes:

(i) O cone tangente de $(f)$ é $\left(Y^{7 b}\right)$.

(ii) $\forall i \geq 1$, mult $\left(a_{i}(X)\right)>i$.

(iii) Para algum $i \geq 1$, mult $\left(a_{i}(X)\right)>i$.

Demonstração: Vamos mostrar que (iii) implica $(i)$. Se existe $i \geq 1$ tal que mult $\left(a_{i}(X)\right)>i$, então $a_{i}(X) Y^{n-i} \notin F_{n}$. Logo,

$$
F_{n}=a_{0}(X) Y^{n}+a_{1}(X) Y^{n-1} \div \ldots+a_{i} \widehat{(X) Y^{n}-i} \div \ldots+a_{n}(X)=(a X+b Y)^{n} .
$$

Como $f$ é regular em $Y$ de ordem $n$, segue que $a_{i}(X)=0, \forall i \geq 1$ e portanto, $F_{n}=Y^{n}$. 
Consideremos $f \in k[[X, Y]]$ irredutível de multiplicidade $n$ e regular em $Y$. Seja $P=Y^{n}+$ $A_{1} Y^{n-1}+\ldots+A_{n} \in k[[X]][Y], A_{i} \in k[[X]], i=1, \ldots, n$, o pseudo-polinômio de grau $n$ associado a $f$ e seja $\alpha=\varphi\left(X^{\frac{1}{n}}\right) \in k\left[\left[X^{\frac{1}{n}}\right]\right]$, onde $n=\min \left\{q \in \mathbb{N} ; \alpha \in k\left[\left[X^{\frac{1}{q}}\right]\right]\right\}$, tal que $P(X, \alpha)=0$. Se colocarmos $T=X^{\frac{1}{n}}$, temos que $\varphi(T) \in k[[T]] \mathrm{e}$

$$
f\left(T^{n}, \varphi(T)\right)=0
$$

Nessas condições dizemos que

$$
\left\{\begin{array}{l}
X=T^{n} \\
Y=\varphi(T)=\sum_{i \geq m} b_{i} T^{i} ; b_{m} \in k \backslash\{0\}
\end{array}\right.
$$

é uma parametrização de Puiseux para o ramo $(f)$.

Outra raiz qualquer de $P$ nos dá uma parametrização de Puiseux $\left(T^{n}, \psi(T)\right)$ de $(f)$, onde $\psi(T)=$ $\varphi(\zeta T)$ e $\zeta$ é uma $n$-ésima raiz da unidade. Estas são as únicas parametrizações de $(f)$ da forma $\left(T^{n}, \varphi(T)\right)$.

Notemos que a condição

$$
n=\min \left\{q \in \mathbb{N} ; \alpha \in k\left[\left[X^{\frac{1}{q}}\right]\right] \text { e } f(X, \alpha)=0\right\}
$$

implica que, em qualquer parametrização de Puiseux como acima, $n$ e os índices $i$ para os quais $b_{i} \neq 0$, são relativamente primos.

Do Teorema 3.3.2(iii), temos

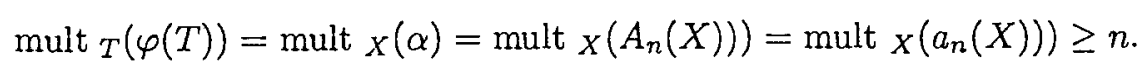

Em particular, se o cone tangente de $(f)$ é dado por $\left(Y^{n}\right)$, temos que

$$
\text { mult } \left.T(\varphi(T))=\text { mult } X\left(a_{n}(X)\right)\right)>n
$$

onde $f$ é como no Lema 3.4.1.

Existem outras parametrizações possíveis de $(f)$ por meio de séries em $k[[T]]$. Seja $\left(\psi_{1}(T), \psi_{2}(T)\right)$ um par de elementos não nulos e não invertíveis em $k[[T]]$. Dizemos que $\left(\psi_{1}(T), \psi_{2}(T)\right)$ é uma parametrização de $(f)$ se

$$
f\left(\psi_{1}(T), \psi_{2}(T)\right)=0
$$

como um elemento de $k[[T]]$.

Uma parametrização $\left(\psi_{1}(T), \psi_{2}(T)\right)$ de $(f)$ é chamada primitiva, se existe um automorfismo $\rho$ de $k[[T]]$ tal que

$$
\left(\rho\left(\psi_{1}(T)\right), \rho\left(\psi_{2}(T)\right)\right)=\left(T^{n}, \varphi(T)\right)
$$

onde $\left(T^{n}, \varphi(T)\right)$ é uma parametrização de Puiseux de $(f)$.

Se $f$ é regular em $X$ temos os mesmos resultados anteriores trocando $X$ por $Y$.

Como poderemos ver nos exemplos a seguir, a relação entre uma curva e sua parametrização de Puiseux é muito complexa. 


\section{Exemplos 3.4.3 1. A curva dada por}

$$
\begin{gathered}
f=Y^{8}-4 X^{3} Y^{6}-8 X^{5} Y^{5}+\left(6 X^{6}-26 X^{7}\right) Y^{4}+\left(-24 X^{9}+16 X^{8}\right) Y^{3}+ \\
+\left(36 X^{10}-4 X^{9}-20 X^{11}\right) Y^{2}+\left(-8 X^{11}+16 X^{12}-8 X^{13}\right) Y+21 X^{14}+X^{12}+6 X^{13}-X^{15}
\end{gathered}
$$

pode ser parametrizada por

$$
\left\{\begin{array}{l}
X=T^{8} \\
Y=T^{12}+T^{14}-T^{15}
\end{array}\right.
$$

2. A curva

$$
(f)=\left(Y^{3}-9 X^{3} Y-X^{4}\right)
$$

tem como parametrização

$$
\left\{\begin{array}{l}
X=T^{3} \\
Y=T^{4}+3 T^{5}-9 T^{7}+27 T^{8}-324 T^{10}+1215 T^{11}-18711 T^{13}+\ldots
\end{array}\right.
$$

Seja $C$ um ramo plano definido por uma série de potências irredutível $f$ de multiplicidade $n$ e regular em $Y$ com parametrização de Puiseux

$$
\left\{\begin{array}{l}
X=T^{n} \\
Y=\varphi(T)=\sum_{i \geq m} b_{i} T^{i} ; b_{m} \neq 0, m>n
\end{array}\right.
$$

Definimos duas sequências finitas $\left(\varepsilon_{i}\right)$ e $\left(\beta_{i}\right)$ de números naturais associados a $(f)$ como segue

$$
\begin{aligned}
& \varepsilon_{0}=\beta_{0}=n \\
& \beta_{j}=\min \left\{i \in \mathbb{N} ; i \neq 0 \bmod \varepsilon_{j-1} \text { e } b_{i} \neq 0\right\}, \text { se } \varepsilon_{j-1} \neq 1 \\
& \varepsilon_{j}=\operatorname{mdc}\left(\varepsilon_{j-1}, \beta_{j}\right)=\operatorname{mdc}\left(\beta_{0}, \ldots, \beta_{j}\right)
\end{aligned}
$$

Observemos que se $\varepsilon_{j-1} \neq 1$, então o conjunto $\left\{i \in \mathbb{N} ; i \neq 0 \bmod \varepsilon_{j-1}\right.$ e $\left.b_{i} \neq 0\right\}$ é não vazio, pois a parametrização é primitiva. Portanto, os $\beta_{j}^{\prime}$ s estão bem definidos com $\beta_{1}$ igual ao primeiro expoente de $T$ em $\varphi(T)$ que não é divisível por $n$ e com coeficiente não nulo. Também temos que $\varepsilon_{j} \mid \varepsilon_{j-1}$, $\forall j \geq 1 \mathrm{e}$

$$
n=\varepsilon_{0}>\varepsilon_{1}>\varepsilon_{2}>\ldots
$$

Consequentemente, para algum $\gamma \in \mathbb{N}$, devemos ter $\varepsilon_{\gamma}=1$, portanto a sequência $\left(\beta_{j}\right)$ é crescente e para em $\beta_{\gamma}$.

Definiçāo 3.4.4 Os expoentes característicos de $C$ são os números naturais $\left(\beta_{0}, \beta_{\mathrm{r}}, \ldots, \beta_{\gamma}\right)$.

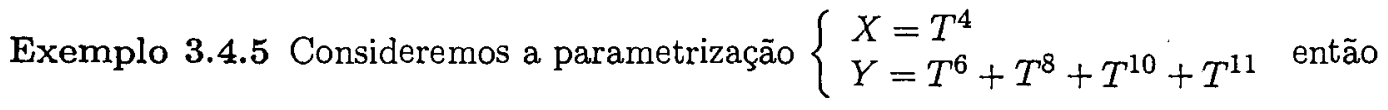

$$
\left\{\begin{array}{l}
\varepsilon_{0}=\beta_{0}=4 \\
\beta_{1}=6, \text { pois } 6 \text { não é divisível por } 4 \\
\varepsilon_{1}=\operatorname{mdc}(4,6)=2 \\
\beta_{2}=11, \text { pois } 6,8 \text { e } 10 \text { são pares } \\
\varepsilon_{2}=\operatorname{mdc}(4,6,11)=1
\end{array}\right.
$$

Portanto, $\gamma=2$. 
Observemos que os expoentes característicos de um ramo plano $C$ determinam os inteiros $\varepsilon_{j}$, pois $\varepsilon_{j}=\operatorname{mdc}\left(\beta_{0}, \ldots, \beta_{j}\right)$.

Com a notação acima, podemos escrever uma parametrização do ramo $\dot{C}$ como segue

$$
\left\{\begin{array}{l}
x=T^{n} \\
y=P\left(T^{n}\right)+\sum_{i=\beta_{1}}^{\beta_{2}-1} b_{i} T^{i}+\ldots+\sum_{i=\beta_{\gamma-1}}^{\beta_{\gamma}-1} b_{i} T^{i}+\sum_{i \geq \beta_{\gamma}} b_{i} T^{i}
\end{array}\right.
$$

onde $P(T) \in k[T]$ e $b_{\beta_{1}}, \ldots, b_{\beta_{\gamma}}$ não são nulos.

Da definição de $\beta_{j}$, podemos deduzir a seguinte propriedade: se $i$ e $j$ são inteiros tais que $\beta_{j-1} \leq i<\beta_{j}$ e se $\varepsilon_{j-1}$ não divide $i$, então $b_{\imath}=0$, pois se $b_{i} \neq 0$, então $i=\beta_{j}$, o que é um absurdo.

Por outro lado, dada qualquer sequência crescente de números naturais relativamente primos $\beta_{0}, \ldots, \beta_{\gamma}$ tais que os inteiros $\varepsilon_{j}$ definidos por $\varepsilon_{j}=\operatorname{mdc}\left(\beta_{0}, \ldots, \beta_{j}\right), j=1, \ldots, \gamma$, formam uma sequência estritamente decrescente, então $\left(\beta_{0}, \ldots, \beta_{\gamma}\right)$ são os expoentes característicos de algum ramo plano $C$.

Também definimos os pares de Puiseux $\left(\eta_{j}, \mu_{j}\right), j=1, \ldots, \gamma$, de $C$, como segue

$$
\eta_{j}=\frac{\varepsilon_{j-1}}{\varepsilon_{j}} \text { e } \mu_{j}=\frac{\beta_{j}}{\varepsilon_{j}}
$$

Como $\varepsilon_{j}=\operatorname{mdc}\left(\varepsilon_{j-1}, \beta_{j}\right)$, temos

$$
\operatorname{mdc}\left(\eta_{j}, \mu_{j}\right)=\operatorname{mdc}\left(\frac{\varepsilon_{j-1}}{\varepsilon_{j}}, \frac{\beta_{j}}{\varepsilon_{j}}\right)=1
$$

\subsection{Curvas analíticas planas}

Seja $\mathbb{C}\{X, Y\}$ o anel de séries de potências em $X$ e $Y$ convergentes com coeficientes complexos. Tal anel tem propriedades muito similares às de $\mathbb{C}[[X, Y]]$. São válidos os Teoremas da Divisão e da Preparação e o Lema de Hensel. Assim, $\mathbb{C}\{X, Y\}$ é um domínio de fatoração única Noetheriano.

Um elemento $f \in \mathbb{C}\{X, Y\}$ é invertível se, e somente se, $f(0,0) \neq 0$. Assim, $\mathbb{C}\{X, Y\}$ é um anel local cujo único ideal maximal é $\mathcal{M}=\langle X, Y)$ satisfazendo

$$
\bigcap_{i \in \mathbb{N}} \mathcal{M}^{i}=\{0\} .
$$

Podemos substituir séries de potências não invertíveis e convergentes, $g_{1}, g_{2} \in \mathbb{C}\{X, Y\}$, em uma série de potências e o resultado ainda será uma série convergente. Também temos que todo automorfismo de $\mathbb{C}\{X, Y\}$ é o resultado da substituição de $X$ e $Y$ por séries $g_{1}$ e $g_{2}$ com multiplicidade 1 e formas lineares linearmente independentes sobre $\mathbb{C}$.

Quando $f$ 'é uma série de potências convergente temos que

$$
C_{f}=\{(z, w) \in U ; f(z, w)=0\}
$$

é uma curva analítica onde $U$ é uma vizinhança suficientemente pequena do zero em $\mathbb{C}^{2}$.

Sejam $f, g \in \mathbb{C}\{X, Y\}$. Seja $U$ uma vizinhança aberta da origem em $\mathbb{C}^{2}$, onde $f$ e $g$ são convergentes. Temos que

$$
C_{f g}=\{(z, w) \in U ; f g(z, w)=0\}=\{(z, w) \in U ; f(z, w)=0\} \cup\{(z, w) \in U ; g(z, w)=0\}=C_{f} \cup C_{g} .
$$


Seja $\phi: \mathbb{C}\{X, Y\} \longrightarrow \mathbb{C}\{X, Y\}$ um $\mathbb{C}$-automorfismo. Então $\phi=\mathcal{S}_{g_{1}, g_{2}}$ e $\phi^{-1}=\mathcal{S}_{h_{1}, h_{2} \text {, }}$ onde $g_{1}, g_{2} \in \mathbb{C}\{X, Y\}$, tem multiplicidade 1 e suas formas lineares são linearmente independentes sobre $\mathbb{C}$ (idem para $h_{1}$ e $h_{2}$ ). Podemos associar a $\phi$ um isomorfismo bi-analitico

$$
\begin{aligned}
& \tilde{\phi}: \quad V \longrightarrow U \\
& (a, b) \longmapsto\left(h_{1}(a, b), h_{2}(a, b)\right)
\end{aligned}
$$

onde $U$ e $V$ são vizinhanças abertas da origem em $\mathbb{C}^{2}$ e

$$
\tilde{\phi}\left(C_{f}\right)=C_{\phi(f)}
$$

De fato, temos que

$$
\widetilde{\phi}\left(C_{f}\right)=\left\{\left(h_{1}(z, w), h_{2}(z, w)\right) ; f(z, w)=0\right\}
$$

Se $f(z, w)=0$, então

$$
\phi(f)\left(h_{1}(z, w), h_{2}(z, w)\right)=f\left(g_{1}\left(h_{1}(z, w), h_{2}(z, w)\right), g_{2}\left(h_{1}(z, w), h_{2}(z, w)\right)=f(z, w)=0\right.
$$

e, portanto, $\tilde{\phi}\left(C_{f}\right) \subset C_{\phi(f)}$ Por outro lado, seja $(\bar{z}, \bar{w}) \in C_{\phi(f)}$, ou seja, $\phi(f)(\bar{z}, \bar{w})=0$. Queremos encontrar $(z, w) \in C_{f}$ tal que $\tilde{\phi}(z, w)=(\bar{z}, \bar{w})$. Para tanto, basta tomar,

$$
(z, w)=\left(g_{1}(\bar{z}, \bar{w}), g_{2}(\bar{z}, \bar{w})\right)
$$

Logo, $C_{\phi(f)} \subset \tilde{\phi}\left(C_{f}\right)$.

Um ponto $P \in C_{f}$ é singular se

$$
f(P)=f_{X}(P)=f_{Y}(P)=0
$$

onde $f_{X}$ e $f_{Y}$ são as derivadas parciais de $f$ com respeito a $X$ e $Y$.

Um ponto $P=(a, b) \in C_{f}$ é regular quando é não singular e, neste caso, definimos a reta tangente a $C_{f}$ passando por $P$ como sendo

$$
T_{P} C_{f}: f_{X}(P)(X-a)+f_{Y}(P)(Y-b)=0 .
$$

Proposição 3.5.1 Seja $f \in \mathbb{C}\{X, Y\}$ reduzida. Entāo existe um aberto $U$ de $\mathbb{C}^{2}$, contendo a origem, tal que $C_{f}$ é regular em $C_{f} \cap(U \backslash\{(0,0)\})$.

\section{Demonstração:}

Como a regularidade (ou singularidade) de um ponto não se altera após uma mudança linear de coordenadas, podemos supor que $f$ é um pseudo-polinômio em uma vizinhança $U^{\prime}$ de $(0,0)$. Séja $g(X)=D_{Y}(f)$ o discriminante de $f$, isto é, a resultante $R_{Y}\left(f, f_{Y}\right)$. Como $f$ é reduzida, segue que $f$ e $f_{Y}$ não possuem fator comum e, portanto, $R_{Y}\left(f, f_{Y}\right)$ não é identicamente nula, pelo Corolário 2.4.3. Como os zeros de uma função analítica $g(X)$ em uma variável são isolados, existe uma vizinhança aberta $V$ da origem em $\mathbb{C}$ tal que

$$
R_{Y}\left(f(a, Y), f_{Y}(a, Y)\right)=g(a) \neq 0, \forall a \in V \backslash\{0\} .
$$


Assim, segue do Corolário 2.4.4 que $f$ e $f_{Y}$ não se anulam simultaneamente em $V \times \mathbb{C} \backslash\{(0,0)\}$. Tomando $U=V \times \mathbb{C}$; o resultado segue.

Analogamente às séries de potências formais, definimos uma relação de equivalência em $\mathbb{C}\{X, Y\}$ : dados $f, g \in \mathbb{C}\{X, Y\}$, dizemos que $(f) \sim(g)$ se, e somente se, existe um $\mathbb{C}$-automorfismo $\dot{\phi}:$ $\mathbb{C}\{X, Y\} \rightarrow \mathbb{C}\{X, Y\}$ e uma unidade $u \in \mathbb{C}\{X, Y\}$ tais que

$$
\phi(f)=u g .
$$

Isto implica que existem vizinhanças $U$ e $V$ da origem em $\mathbb{C}^{2}$ tais que $C_{f}$ está definida em $U, C_{g}$ está definida em $V$ e a aplicação bi-analítica associada $\widetilde{\phi}: U \longrightarrow V$ satisfaz $\widetilde{\phi}\left(C_{f}\right)=C_{g}$.

Por outro lado, vamos mostrar que se $f, g \in \mathbb{C}\{X, Y\}$ são reduzidas e se existe uma aplicação bi-analítica $\widetilde{\phi}: U \longrightarrow V$, onde $U$ e $V$ são vizinhanças abertas da origem em $\mathbb{C}^{2}$, tal que $\widetilde{\phi}\left(C_{f}\right)=C_{g}$, então $(f) \sim(g)$.

Observamos que o Teorema de Newton-Puiseux, neste contexto, diz que se $f \in \mathbb{C}\{X, Y\}$ é um pseudo-polinômio irredutível de multiplicidade $n$, então existe uma série de potências $\varphi(t) \in \mathbb{C}\{t\}$ convergente em, um disco $D$ centrado na origem de $\mathbb{C}$, tal que

$$
f\left(t^{n}, \varphi(t)\right)=0, \forall t \in D \text {. }
$$

Isto nos dá uma parametrização de $C_{f}$ perto da origem em $\mathbb{C}^{2}$ por funções analíticas. Por outro lado, existem $n$ parametrizações de $C_{f}$, digamos $\left(t^{n}, \varphi_{i}(t)\right), i=0, \ldots, n-1$, onde $\varphi_{i}(t)=\varphi\left(\zeta^{i} t\right)$ e $\zeta$ é uma raiz $n$-ésima primitiva da unidade. Deste modo, $\forall z \in \mathbb{C}$ próximo da origem, se $w$ é uma $n$-ésima raiz de $z$, então $\varphi_{0}(w), \ldots, \varphi_{n-1}(w)$ são raízes do polinômio $f(z, Y)$. Como $D_{Y}(f) \neq 0$, as raízes em $\mathbb{C}$ de $f(z, Y)$ para $z \neq 0$ próximo da origem são todas distintas. Isto mostra que, para $z \neq 0$, se $\varphi(z)=\varphi_{i}(z)$, então $i=0$.

Lema 3.5.2 Seja $f \in \mathbb{C}\{X\}[Y]$ um pseudo-polinômio irredutivel de multiplicidade n. Então existem uma vizinhança aberta $U$ de $\mathbb{C}^{2}$ contendo a origem, um disco $D$ centrado na origem de $\mathbb{C}$ e uma aplicação analítica

$$
\begin{aligned}
\Psi: D & \longrightarrow U \\
t & \longmapsto \Psi(t)=\left(t^{n}, \varphi(t)\right)
\end{aligned}
$$

tais que $f$ é convergente em $U, \Psi(D)=C_{f} \cap U$ e $\Psi: D \longrightarrow \Psi(D)$ é um homeomorfismo com $\Psi(0)=(0,0)$.

\section{Demonstração:}

Seja $D_{1}$ um disco centrado na origem de $\mathbb{C}$ onde os coeficientes de $f$ em $\mathbb{C}\{X\}$ convergem e $D_{Y}(f)(z) \neq 0, \forall z \in D_{1} \backslash\{0\}$. Sejam $\varphi$ e $D$ como acima, com $D$ suficientemente pequeno de modo que $t^{n} \in D_{1}, \forall t \in D$. Seja $U=D_{1} \times \mathbb{C}$. Temos que $\Psi(D) \subset C_{f} \cap U$. Reciprocamente, seja $(z, w) \in C_{f} \cap U$, Então existe $t \in D$ tal que $z=t^{n}$ e $w=\varphi\left(\zeta^{i} t\right)$, para algum $i=0, \ldots, n$. Portanto $\Psi(D)=C_{f} \cap U$. Ainda, $\Psi$ é injetiva, pois se $\Psi(t)=\Psi\left(t^{\prime}\right)$, então $t^{\prime}=\zeta^{i} t$, para algum $i$, onde $\zeta$ é uma raiz $n$-ésima primitiva da unidade. Portanto

$$
\varphi(t)=\varphi\left(t^{\prime}\right)=\varphi\left(\zeta^{i} t\right)=\varphi_{i}(t)
$$

O que implica pela observação acima que $i=0 \mathrm{e}$, portanto, $t=t^{\prime}$. 
A aplicação $\Psi$ é analítica e $\left.\Psi\right|_{D \backslash\{0\}}$ tem derivada não nula em todos os pontos. Logo, $\Psi$ é um homeomorfismo entre $D \backslash\{0\}$ e $\left(C_{f} \cap U\right) \backslash\{(0,0)\}$, que por continuidade pode ser estendido a um homeomorfismo entre $D$ e $C_{f} \cap U$.

Corolário 3.5.3 Se $f \in \mathbb{C}\{X, Y\}$ é irredutível, então $C_{f}$ é homeomorfo a um dịsco numa vizinhança da origem.

Lema 3.5.4 Se $f$ e $g$ são irredutíveis e não associados em $\mathbb{C}\{X, Y\}$ então existe uma vizinhança $U$ da origem em $\mathbb{C}^{2}$ tal que

$$
C_{f} \cap C_{g} \cap U=\{(0,0)\}
$$

\section{Demonstração:}

Podemos reduzir o problema para o caso em que $f$ e $g$ são pseudo-polinômios.

Como $f$ e $g$ são irredutíveis e não associados, segue do Corolário 2.4 .3 que $R_{Y}(f, g)$ não é identicamente nula. Logo, existe uma vizinhança $D$ da origem em $\mathbb{C}$ tal que $R_{Y}(f, g)(a) \neq 0, \forall a \in D \backslash\{0\}$. Isto implica que $f(a, Y)$ e $g(a, Y)$ não possuem raízes em comum em $\mathbb{C}, \forall a \in D \backslash\{0\}$. Tomando $U=D \times \mathbb{C}$, o resultado segue.

Corolário 3.5.5 Sejam $f$ e $g$ em $\mathbb{C}\{X, Y\}$ irredutiveis. Então $C_{f}=. C_{g}$ se, e somente se, $f$ e $g$ são associados.

\section{Demonstração:}

Se $f$ e $g$ são associados, temos por definição que $C_{f}=C_{g}$.

A recíproca segue do Lema 3.5.4, pois se $f$ e $g$ não são associados então $C_{f} \cap C_{g} \cap U=\{(0,0)\}$.

Proposição 3.5.6 Sejam $f$ e $g$ em $\mathbb{C}\{X, Y\}$ e suponhamos que exista uma vizinhança da origem em $\mathbb{C}^{2}$ tal que $C_{f}=C_{g}$, então red $(f)$ e red $(g)$ säo associados.

\section{Demonstração:}

Sejam $f=f_{1}^{n_{1}} \ldots f_{r}^{n_{r}}$ e $g=g_{1}^{m_{1}} \ldots g_{s}^{m_{s}}$. Temos que red $(f)=f_{1} \ldots f_{r}$, red $(g)=g_{1} \ldots g_{s}$ e

$$
C_{f}=C_{f_{1}} \cup \ldots \cup C_{f_{r}} \text { e } C_{g}=C_{g_{1}} \cup \ldots \cup C_{g_{s}} .
$$

Segue do Corolário 3.5.3 e do Lema 3.5.4 que, numa vizinhança da origem, $C_{f_{i}} \backslash\{(0,0)\}, i=1, \ldots, r$, e $C_{g_{j}} \backslash\{(0,0)\}, j=1, \ldots, s$, são respectivamente as componentes conexas de $C_{f} \backslash\{(0,0)\}$ e $C_{g} \backslash\{(0,0)\}$. Como $C_{f}=C_{g}$ então $r=s$ e $C_{f_{i}}=C_{g_{j}}$, para algum $j$. Segue do Corolário 3.5 .5 que $\left(f_{i}\right)$ e $\left(g_{j}\right)$ são associados para algum $j$. Portanto, red $(f)$ e red $(g)$ são associados.

Corolário 3.5.7 Sejam $f$ e $g$ em $\mathbb{C}\{X, Y\}$ reduzidas. Sejam $U$ e $V$ vizinhanças da origem tais que $f$ é convergente em $U$ e $g$ é convergente em $V$. Suponhamos que exista uma função bi-analítica $\widetilde{\phi}: U \longrightarrow V$ tal que $\widetilde{\phi}\left(C_{f}\right)=C_{g}$. Então $(f) \sim(g)$. 


\section{Demonstração:}

Consideremos $\tilde{\phi}^{-1}: V \longrightarrow U$ dada por $\widetilde{\phi}^{-1}(a, b)=\left(h_{1}(a, b), h_{2}(a, b)\right)$, onde $h_{1}, h_{2} \in \mathbb{C}\{X, Y\}$ tem multiplicidade 1 e formas lineares linearmente independentes sobre $\mathbb{C}$.

Definimos $\psi: \mathbb{C}\{X, Y\} \longrightarrow \mathbb{C}\{X, Y\}$ dada por $\psi=S_{h_{1}, h_{2}}$. Logo, $\psi$ é um $\mathbb{C}$-automorfismo e sua inversa $\phi$ é o automorfismo de $\mathbb{C}\{X, Y\}$ associado a $\widetilde{\phi}$. Temos que $\widetilde{\phi}\left(C_{f}\right)=C_{\phi(f)}$ e por hipótese $\widetilde{\phi}\left(C_{f}\right)=C_{g}$. Portanto, $C_{\phi(f)}=C_{g}$ e segue da Proposição 3.5.6 que red( $\left.\phi(f)\right)$ e $\operatorname{red}(g)$ são associados em alguma vizinhança da origem em $\mathbb{C}^{2}$. Como $f$ é reduzida, segue que $\phi(f)$ é reduzida e, como $g$ também é reduzida, segue que $\phi(f)$ e $g$ são associados.

No que segue, vamos dar uma interpretação geométrica para o cone tangente de uma curva.

Definição 3.5.8 O espaço projetivo $\mathbb{C P}^{n-1}$ associado a $\mathbb{C}^{n}$ é o quociente de $\mathbb{C}^{n} \backslash\{0\}$ módulo a relação de equivalência: se $z, z^{\prime} \in \mathbb{C}^{n} \backslash\{0\}$, então $z$ é equivalente a $z^{\prime}$ se existe $\lambda \in \mathbb{C} \backslash\{0\}$ tal que $z=\lambda z^{\prime}$.

Denotaremos por $\left(x_{0}: \ldots: x_{n-1}\right)$ a classe de equivalência de $\left(x_{0}, \ldots, x_{n-1}\right) \in \mathbb{C}^{n} \backslash\{0\}$ e chamaremos de coordenadas homogêneas. Os pontos da reta $b_{0} X-a_{0} Y=0$ em $\mathbb{C}^{2}$ estão na classe $\left(a_{0}: b_{0}\right)$ em $\mathbb{C P}^{1}$.

Proposição 3.5.9 Seja $C_{f}$ uma curva analítica numa vizinhança $U$ da origem em $\mathbb{C}^{2}$. Seja $\left(P_{m}\right)$ uma sequência de pontos em $\dot{C}_{f} \backslash\{0\}$ que converge para a origem. Então a sequência $\left(\overline{P_{m}}\right)$ de retas complexas $\overline{P_{m}}$ passando pela origem e por $P_{m}$, vistas como pontos em $\mathbb{C P}^{1}$, converge para $\bar{P}$, que ë uma reta pertencente ao cone tangente de $C_{f}$.

\section{Demonstração:}

Seja

$$
f(X, Y)=F_{n}(X, Y)+F_{n+1}(X, Y)+\ldots
$$

a expansão em série de Taylor de $f$ onde $F_{j}$ é um polinômio homogêneo de grau $j$ e $F_{n} \neq 0$. $O$ cone tangente de $C_{f}$ é dado por $F_{n}(X, Y)=0$.

Suponhamos $P_{m}=\left(a_{m}, b_{m}\right) \in C_{f} \backslash\{0\}, m \in \mathbb{N}$. Como $P_{m} \neq(0,0)$ vamos assumir, sem perda de de generalidade, que $a_{m} \neq 0$. Mas

$$
\mathbb{C P}^{1}=\{(x: y) ; x \neq 0\} \cup\{(x: y) ; y \neq 0\}
$$

e como $\{(x: y) ; x \neq 0\}$ é aberto em $\mathbb{C P}^{1}$, existe $m_{0} \in \mathbb{N}$ tal que $\forall m \geq m_{0},\left(a_{m}: b_{m}\right) \in\{(x: y) ; x \neq 0\}$. Portanto,

$$
0=f\left(P_{m}\right)=F_{n}\left(a_{m}, b_{m}\right)+F_{n+1}\left(a_{m}, b_{m}\right)+\ldots=a_{m}^{n}\left(F_{n}\left(1, \frac{b_{m}}{a_{m}}\right)+\sum_{i \geq 1} a_{m}^{i} F_{n+i}\left(1, \frac{b_{m}}{a_{m}}\right)\right)
$$

para $m \geq m_{0}$. Logo

$$
F_{n}\left(1, \frac{b_{m}}{a_{m}}\right)+\sum_{i \geq 1} a_{m}^{i} F_{n+i}\left(1, \frac{b_{m}}{a_{m}}\right)=0
$$

pois $a_{m} \neq 0$.

Tomando o limite quando $m \rightarrow \infty$ temos que $a_{m} \rightarrow 0$, logo

$$
F_{n}\left(1, \frac{b_{m}}{a_{m}}\right) \rightarrow 0 \text {. }
$$


Portanto, $\left(1: \frac{b_{m}}{a_{m}}\right) \rightarrow\left(1: \frac{b}{a}\right) \operatorname{com}\left(1: \frac{b}{a}\right)$ pertencente ao cone tangente. Assim, $F_{n}(a, b)=0$ e $\overline{P_{m}} \rightarrow \bar{P}$, com $\bar{P}$ pertencente ao cone tangente.

Proposição 3.5.10 Seja C uma curva analítica plana irredutível definida numa vizinhança da origem e regular fora da origem. Para qualquer sequência de pontos $\left(P_{m}\right)$ em $\mathbb{C}^{2} \backslash\{0\}$ que converge para a origem temos que $T_{P_{m}} C$ tende ao cone tangente de $C$.

\section{Demonstração:}

Podemos supor que a equação de $C$ é regular em $Y$ e que sua reta tangente é dada por $(Y)$. Temos que $C$ tem uma parametrização da forma $\left(T^{n}, \varphi(T)\right)$, onde $n=$ mult $(C)$ e

$$
\varphi(T)=b_{r} T^{r}+b_{r+1} T^{r+1}+\ldots \in \mathbb{C}\{T\} .
$$

Um vetor diretor da reta tangente a $C$ no ponto $P_{m}=\left(t_{m}^{n}, \varphi\left(t_{m}\right)\right)$ é dado por

$$
\left(n t_{m}^{n-1}, \frac{\partial \varphi}{\partial T}\left(t_{m}\right)\right)=\left(n t_{m}^{n-1}, r b_{r} t_{m}^{r-1}+(r+1) b_{r+1} t_{m}^{r}+\ldots\right) .
$$

Vamos considerar o vetor

$$
\left(1, \frac{r}{n} b_{r} t_{m}^{r-n}+\frac{r+1}{n} b_{r+1} t_{m}^{r-n+1}+\ldots\right) .
$$

Como mult $T(\varphi(T))=r>n$, então $r-n>0$. Quando $t_{m} \rightarrow 0$, as retas tạngentes tendem para $Y=0$, que é a reta tangente de $C$. 


\section{Capítulo 4}

\section{Interseção de Curvas}

\subsection{O anel local de uma curva plana}

Dado um anel $A$ e elementos $z_{1}, \ldots, z_{n} \in A$, denotamos por $\left\langle z_{1}, \ldots, z_{n}\right\rangle$ o ideal de $A$ gerado pelos elementos $z_{1}, \ldots, z_{n}$.

Seja $k$ um corpo qualquer e $f$ um elemento no ideal maximal $\mathcal{M}=\langle X, Y\rangle$ de $k[[X, Y]]$.

Definimos o anel de coordenadas de uma curva $(f)$ como sendo a $k$-álgebra

$$
\mathcal{O}_{f}=\frac{k[[X, Y]]}{\langle f\rangle}
$$

Se $h \in k[[X, Y]]$ e $B \subset k[[X, Y]]$, denotaremos por $\bar{h}$ a classe de $h$ em $\mathcal{O}_{f}$ e por $\bar{B}$ o conjunto das classes dos elementos de $B$. Denotaremos a classe residual $\bar{Y}$ por $y$ e $\bar{X}$ por $x$, respectivamente.

$\mathrm{O}$ anel $\mathcal{O}_{f}$ é local com ideal maximal

$$
\mathcal{M}_{f}=\overline{\mathcal{M}}
$$

Quando $f$ é irredutível, temos que $\mathcal{O}_{f}$ é um domínio e, neste caso, indicaremos o corpo de frações de $\mathcal{O}_{f}$ por $K_{f}$.

$\mathrm{O}$ anel $\mathcal{O}_{f}$ é um importante invariante das classes de equivalência de curvas algebróides planas. Quando as $k$-álgebras locais $\mathcal{O}_{f}$ e $\mathcal{O}_{g}$ forem isomorfas, denotaremos por $\mathcal{O}_{f} \cong \mathcal{O}_{g}$.

Teorema 4.1.1 Sejam $(f)$ e $(g)$ duas curvas algebróides planas. Então $(f) \sim(g)$ se, e somente se, $\mathcal{O}_{f} \cong \mathcal{O}_{g}$

\section{Demonstração:}

Seja $h \in k[[X, Y]]$. Vamos uenotar por $\bar{h}=h+\langle f\rangle$ e $\overline{\bar{h}}=h+\langle g\rangle$. Suponhamos $(f) \sim(g)$. Então existem um $k$-automorfismo $\Phi$ e uma unidade $u \in k[[X, Y]]$ tais que

$$
\Phi(f)=u g .
$$

Se $\pi: k[[X, Y]] \longrightarrow \mathcal{O}_{g}$ é projeção canônica então $\pi \circ \Phi$ é sobrejetora e $\operatorname{ker}(\pi \circ \Phi)=\langle f\rangle$. De fato, claramente, $\langle f\rangle$ ker $\subset(\pi \circ \Phi)$. Reciprocamente, se $h \in \operatorname{ker}(\pi \circ \Phi)$, então $\Phi(h) \in\langle g\rangle=\langle\Phi(f)\rangle$. Como $\Phi$ é um automorfismo, segue que $h=\Phi^{-1}\left(h_{0}\right) f$, para algum $h_{0} \in k[[X, Y]]$. Portanto, $h \in\langle f\rangle$. Assim, $\pi \circ \Phi$ induz um isomorfismo entre $\mathcal{O}_{f}$ e $\mathcal{O}_{g}$. 
Suponhamos agora que $\mathcal{O}_{f} \cong \mathcal{O}_{g}$. Se mult $(f)=\operatorname{mult}(g)=1$, segue da Proposição 3.1.3 que $(f) \sim(g)$. Suponhamos que mult $(g) \geq 2$. Sejam $\widetilde{\Phi}: \mathcal{O}_{f} \longrightarrow \mathcal{O}_{g}$ um isomorfismo e $T_{1}, T_{2} \in \mathcal{M}$,

$$
\begin{aligned}
& T_{1}=(a X+b Y)+\ldots \\
& T_{2}=(c X+d Y)+\ldots
\end{aligned}
$$

tais que

$$
\left\{\begin{array}{l}
\widetilde{\Phi}(\bar{X})=\overline{\overline{T_{1}}} \\
\widetilde{\Phi}(\bar{Y})=\overline{\overline{T_{2}}}
\end{array}\right.
$$

Definimos o homomorfismo de substituição

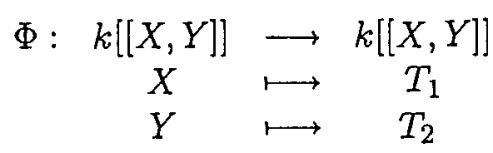

Como $\widetilde{\Phi}$ é um isomorfismo, existem $R(X, Y), S(X, Y) \in k[[X, Y]]$,

$$
\begin{aligned}
& R(X, Y)=a_{r} X+b_{r} Y+R_{2}+R_{3}+\ldots \\
& S(X, Y)=a_{s} X+b_{s} Y+S_{2}+S_{3}+\ldots
\end{aligned}
$$

tais que

$$
\begin{aligned}
& \overline{\bar{X}}=\widetilde{\Phi}(R(\bar{X}, \bar{Y}))=R\left(\overline{\overline{T_{1}}}, \overline{\overline{T_{2}}}\right) \\
& \overline{\bar{Y}}=\widetilde{\Phi}(S(\bar{X}, \bar{Y}))=S\left(\overline{\overline{T_{1}}}, \overline{\overline{T_{2}}}\right)
\end{aligned}
$$

Isto implica que

$$
\begin{aligned}
& X-R\left(T_{1}, T_{2}\right) \in\langle g\rangle \subset \mathcal{M}^{2} \\
& Y-S\left(T_{1}, T_{2}\right) \in\langle g\rangle \subset \mathcal{M}^{2}
\end{aligned}
$$

Como,

$$
\begin{aligned}
& R\left(T_{1}, T_{2}\right)=a_{r}((a X+b Y)+\ldots)+b_{r}((c X+d Y)+\ldots)+\ldots \\
& S\left(T_{1}, T_{2}\right)=a_{s}((a X+b Y)+\ldots)+b_{s}((c X+d Y)+\ldots)+\ldots
\end{aligned}
$$

Temos que os sistemas

$$
\left\{\begin{array} { l } 
{ a _ { r } a + b _ { r } c = 1 } \\
{ a _ { r } b + b _ { r } d = 0 }
\end{array} \quad \text { e } \left\{\begin{array}{l}
a_{s} a+b_{s} c=0 \\
a_{s} b+b_{s} d=1
\end{array}\right.\right.
$$

têm solução única, o que implica que $a d-b c \neq 0$. Assim, as formas lineares de $T_{1}$ e $T_{2}$ são linearmente independentes e segue da Proposição 1.2.7 que $\Phi$ é um $k$-automorfismo tal que

$$
\overline{\overline{0}}=\widetilde{\Phi}(f(\bar{X}, \bar{Y}))=f\left(\overline{\overline{T_{1}}}, \overline{\overline{T_{2}}}\right) .
$$

Logo, $\Phi(f) \in\langle g\rangle$, isto é, existe $h \in k[[X, Y]]$ tal que $\Phi(f)=h g$.

Em particular,

$$
\text { mult }(f)=\operatorname{mult}(\Phi(f))=\text { mult }(h)+\text { mult }(g) \geq 2 .
$$

Sejam $S_{1}, S_{2} \in k[[X, Y]]$ tais que

$$
\begin{array}{ccc}
\Phi^{-1}: k[[X, Y]] & \longrightarrow k[[X, Y]] \\
X & \longmapsto & S_{1} \\
Y & \longmapsto & S_{2}
\end{array}
$$


Então $\Phi^{-1}(g)=g\left(S_{1}, S_{2}\right)$ e $\overline{\Phi^{-1}(g)}=g\left(\overline{S_{1}}, \overline{S_{2}}\right) \in \mathcal{O}_{f}$. Portanto,

$$
\widetilde{\Phi}\left(g\left(\overline{S_{1}}, \overline{S_{2}}\right)\right)=g\left(S_{1}\left(\overline{\overline{T_{1}}}, \overline{\overline{T_{2}}}\right), S_{2}\left(\overline{\overline{T_{1}}}, \overline{\overline{T_{2}}}\right)\right)=g(\overline{\bar{X}}, \overline{\bar{Y}})=0 .
$$

Logo, $\Phi^{-1}(g) \in\langle f\rangle$, isto é, existe $h^{\prime} \in k[[X, Y]]$ tal que $\Phi^{-1}(g)=h^{\prime} f$.

Assim, $g=\Phi \circ \Phi^{-1}(g)=\Phi\left(h^{\prime}\right) \Phi(f)=\Phi\left(h^{\prime}\right) h g$, o que implica que $h$ é uma unidade. Segue que $(f) \sim(g)$.

Vimos no teorema acima que se as $k$-álgebras $\mathcal{O}_{f}$ e $\mathcal{O}_{g}$ são isomorfas então $(f)$ e $(g)$ são equivalentes. Assim, temos o seguinte corolário.

Corolário 4.1.2 Se $\mathcal{O}_{f} \cong \mathcal{O}_{g}$ então mult $(f)=\operatorname{mult}(g)$.

Proposição 4.1.3 Seja $f \in \mathcal{M}$ regular em $Y$ de ordem n. Então $\mathcal{O}_{f}$ é um $k[[X]]-$ módulo livre de rank $n$ gerado pelas classes $y^{i}$ de $Y^{i}, i=0, \ldots, n-1, e m \mathcal{O}_{f}$. Em outras palavras,

$$
\mathcal{O}_{f}=k[[X]] \oplus k[[X]] y \oplus \ldots \oplus k[[X]] y^{n-1} .
$$

\section{Demonstração:}

Seja $g \in k[[X, Y]]$. Aplicando o Teorema da Divisão 2.1.3, podemos encontrar $q \in k[[X, Y]] \mathrm{e}$ $r \in k[[X]][Y], \partial_{Y} r<n$, tais que $g=q f+r$. Então existem $a_{0}(X), \ldots, a_{n-1}(X) \in k[[X]]$ tais que

$$
g=q f+a_{0}(X)+a_{1}(X) Y+\ldots+a_{n-1}(X) Y^{n-1} .
$$

Logo,

$$
\bar{g}=a_{0}(X)+a_{1}(X) y+\ldots+a_{n-1}(X) y^{n-1}
$$

pois $f$ é regular em $Y$ de ordem $n$. Portanto, $\mathcal{O}_{f}$ é um $k[[X]]-$ módulo gerado por $1, y, \ldots, y^{n-1}$.

Mostremos que estes elementos são livres sobre $k[[X]]$. Suponhamos que exista uma relação não trivial em $\mathcal{O}_{f}$, com coeficientes em $k((X)$.)

$$
b_{0}(X)+b_{1}(X) y+\ldots+b_{n-1}(X) y^{n-1}=0 .
$$

Logo, existe $q \in k[[X]][Y]$ tal que

$$
b_{0}(X)+b_{1}(X) Y+\ldots+b_{n-1}(X) Y^{n-1}=q f .
$$

Como $X$ não divide $f$, segue que, a menos de uma possivel divisão dos $b_{i}^{\prime}$ s e de $q$ por $X$, podemos supor que $b_{j}(0) \neq 0$ para algum $j=0, \ldots, n-1$. Tomando $X=0$ na expressão acima temos

$$
b_{0}(0)+b_{1}(0) Y+\ldots+b_{n-1}(0) Y^{n-1}=q(0, Y) f(0, Y) .
$$

Como $Y^{n} \mid f(0, Y) \neq 0$, segue que $b_{0}(0)=b_{1}(0)=\ldots=b_{n-1}(0)=q(0, Y)=0$, o que contradiz 0 fato de que $b_{j}(0) \neq 0$ para algum $j$.

Da Proposição acima deduzimos a seguinte propriedade fundamental das curvas representadas por séries de potências irredutíveis regulares em $Y$ de ordem $n$ e com uma parametrização do tipo $\left(T^{n}, \varphi(T)\right)$ 
Consideremos o homomorfismo

$$
\begin{aligned}
\alpha: k[[X, Y]] & \longrightarrow k[[T]] \\
g & \longmapsto g\left(T^{n}, \varphi(T)\right)
\end{aligned}
$$

Seja $g \in \operatorname{ker}(\alpha)$. Pelo Teorema da Divisão 2.1.3, existem $q \in k[[X, Y]]$ e $r \in k[[X]][Y], \partial_{Y} r<n$, tais que $g=q f+r$.

Como $g\left(T^{n}, \varphi(T)\right)=0$, com $\varphi(T) \in k[[T]]$, segue que $q\left(T^{n}, \varphi(T)\right) f\left(T^{n}, \varphi(T)\right)+r\left(T^{n}, \varphi(T)\right)=0$. Mas, $f\left(T^{n}, \varphi(T)\right)=0$. Portanto, $r\left(T^{n}, \varphi(T)\right)=0$. Logo, $r$ é divisível pelo polinômio minimal de $\varphi\left(X^{\frac{1}{n}}\right)$, que tem grau $n$. Então, $r=0$ e $g=q f$. Assim, $\operatorname{ker}(\alpha)=\langle f\rangle$.

Portanto, $\alpha$ induz um homomorfismo injetor

$$
\begin{aligned}
H_{\varphi}: \mathcal{O}_{f} & \longrightarrow k[[T]] \\
\bar{g} & \longmapsto g\left(T^{n}, \varphi(T)\right)
\end{aligned}
$$

que nos permite identificar $\mathcal{O}_{f}$ com a subálgebra de $k[[T]]$, dada por

$$
A_{\varphi}:=H_{\varphi}\left(\mathcal{O}_{f}\right)=k\left[\left[T^{n}, \varphi(T)\right]\right]
$$

Se $\psi(T)=\varphi(\zeta T)$, onde $\zeta$ é uma $n$-ésima raiz da unidade, então $A_{\psi} \cong A_{\varphi}$, pelo automorfismo

$$
\begin{aligned}
h_{\zeta}: k[[T]] & \longrightarrow k[[T]] \\
P(T) & \longmapsto P(\zeta T)
\end{aligned}
$$

Segue da Proposição 4.1.3 que

$$
A_{\varphi}=k\left[\left[T^{n}\right]\right] \oplus k\left[\left[T^{n}\right]\right] \varphi \oplus \ldots \oplus k\left[\left[T^{n}\right]\right] \varphi^{n-1} .
$$

O corpo de frações de $A_{\varphi}$ é $k((T)$ ) (ver Teorema 3.3.2(1)).

Os resultados acima podem ser obtidos quando $f$ é regular em $X$ (Basta trocar $Y$ por $X$ ).

Teorema 4.1.4 Seja $f \in k[[X]][Y]$ um pseudo-polinômio irredutivel de grau $n$ e seja $\varphi$ um elemento de $k\left[\left[X^{\frac{1}{n}}\right]\right]$ tal que $f(X, \varphi)=0$. Se $D_{Y}(f)(X) \in k[[X]]$ é o discriminante de $f(X, Y)$, então

$$
D_{Y}(f)\left(T^{n}\right) k[[T]] \subset A_{\varphi}
$$

\section{Demonstração:}

Considere o seguinte diagrama

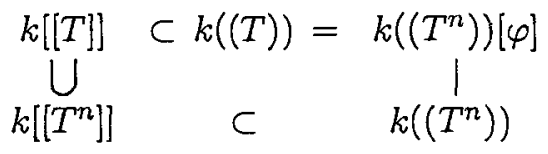

Temos que $k\left(\left(T^{n}\right)\right)[\varphi] / k\left(\left(T^{n}\right)\right)$ é uma extensão galoisiana com grupo de Galois isomorfo a $U_{n}$ (ver Teorema 3.2.2).

Seja $\beta \in k[[T]]$. Como um elemento de $k((T))=k\left(\left(T^{n}\right)\right)[\varphi]$, (ver Teorema 3.3.2), podemos escrever $\beta$ na forma

$$
\beta=\sum_{i=0}^{n-1} a_{i}\left(T^{n}\right) \varphi^{i} ; \quad a_{i}\left(T^{n}\right) \in k\left(\left(T^{n}\right)\right)
$$


Seja $\zeta$ um gerador do grupo cíclico $U_{n}$. Seja $\beta_{j}=\zeta^{j} * \beta$ e $\varphi_{j}=\zeta^{j} * \varphi$ (ver Seção. 3.3). Aplicando $\zeta^{j}$ à $\beta$, obtemos o sistema

$$
\beta_{j}=\sum_{i=0}^{n-1} a_{i}\left(T^{n}\right) \varphi_{j}^{i}, j=0, \ldots, n-1 .
$$

cujo determinante é o determinante de Vandermonde (ver [3], pag. 258)

$$
\operatorname{det}\left(\begin{array}{cccc}
\varphi_{0}^{0} & \varphi_{0}^{1} & \ldots & \varphi_{0}^{n-1} \\
\varphi_{1}^{0} & \varphi_{1}^{1} & \ldots & \varphi_{1}^{n-1} \\
\vdots & \vdots & \ldots & \vdots \\
\varphi_{n-1}^{0} & \varphi_{n-1}^{1} & \ldots & \varphi_{n-1}^{n-1}
\end{array}\right)=\prod_{r<s}\left(\varphi_{s}-\varphi_{r}\right)=\Delta
$$

Da Proposição 2.4.9, segue que $\Delta^{2}= \pm D_{Y}(f)\left(T^{n}\right)$. Aplicando a Regra de Cramer ao sistema acima obtemos

$$
a_{i}\left(T^{n}\right)=\Delta^{-1} M_{i}
$$

onde

$$
M_{i}=\operatorname{det}\left(\begin{array}{ccccc}
\varphi_{0}^{0} & \ldots & \beta_{0} & \ldots & \varphi_{0}^{n-1} \\
\varphi_{1}^{0} & \ldots & \beta_{1} & \ldots & \varphi_{1}^{n-1} \\
\vdots & \ldots & \vdots & \ldots & \vdots \\
\varphi_{n-1}^{0} & \ldots & \beta_{n-1} & \ldots & \varphi_{n-1}^{n-1}
\end{array}\right) \in k[[T]]
$$

é obtido substituindo a $i$-ésima coluna por $\beta_{0}, \ldots, \beta_{n-1}$. Então $\Delta^{2} a_{i}\left(T^{n}\right)=\Delta M_{i} \in k\left[\left[T^{n}\right]\right]$. Como $\beta \in k[[T]]$, temos que

$$
D_{Y}(f)\left(T^{n}\right) \beta= \pm \sum_{i=0}^{n-1} \Delta^{2} a_{i}\left(T^{n}\right) \varphi^{i} \in \sum_{i=0}^{n-1} k\left[\left[T^{n}\right]\right] \varphi^{i}=A_{\varphi}
$$

Logo, $D_{Y}(f)\left(T^{n}\right) k[[T]] \subset A_{\varphi}$.

Corolário 4.1.5 Com a mesma notação do teorema acima, temos que

$$
\operatorname{dim}_{k} \frac{k[[T]]}{A_{\varphi}}<\infty
$$

\section{Demonstração:}

Temos que

$$
\operatorname{dim}_{k} \frac{k[[T]]}{A_{\varphi}}<\operatorname{dim}_{k} \frac{k[[T]]}{D_{Y}(f)\left(T^{n}\right) k[[T]]}=\operatorname{dim}_{k} \frac{k[[T]]}{\left\langle D_{Y}(f)\left(T^{n}\right)\right\rangle}=\operatorname{mult}\left(D_{Y}(f)\left(T^{n}\right)\right)<\infty .
$$

Segue do corolário acima que existe um número natural $c$ tal que se $h(T) \in k[[T]]$ é tal que mult $(h(T)) \geq c$, então $h(T) \in A_{\varphi}$. Identificando $A_{\varphi}$ com $\mathcal{O}_{f}$, podemos dizer que $\mathcal{O}_{f}$ tem um condutor que é o menor inteiro $c$ com esta propriedade. Tal inteiro é chamado de condutor da curva $(f)$. 
Definimos a valorização associada a $f$ como sendo a função

$$
\begin{aligned}
v_{f}: \mathcal{O}_{f} \backslash\{0\} & \longrightarrow \mathbb{N} \\
\bar{g} & \longmapsto \operatorname{mult}\left(H_{\varphi}(\bar{g})\right)=\operatorname{mult}\left(g\left(T^{n}, \varphi(T)\right)\right)
\end{aligned}
$$

e $v_{f}(\overline{0})=\infty$.

Seja $\left(\psi_{1}(T), \psi_{2}(T)\right)$ é uma parametrização primitiva de $f$. Logo existe um automorfismo $\rho$ de $k[[T]]$ tal que

$$
\left(\rho\left(\psi_{1}(T), \psi_{2}(T)\right)\right)=\left(T^{n}, \varphi(T)\right)
$$

Assim,

$$
\begin{gathered}
v_{f}(g)=\operatorname{mult}\left(g\left(T^{n}, \varphi(T)\right)\right)=\operatorname{mult}\left(g\left(\rho \psi_{1}(T), \rho \psi_{2}(T)\right)\right)= \\
\text { mult }\left(\rho g\left(\psi_{1}(T), \psi_{2}(T)\right)=\operatorname{mult}\left(g\left(\psi_{1}(T), \psi_{2}(T)\right)\right)\right.
\end{gathered}
$$

Como consequência das propriedades da multiplicidade temos que a valorização $v_{f}$ satisfaz as seguinte propriedades

- $v_{f}(\bar{g} \bar{h})=v_{f}(\bar{g})+v_{f}(\bar{h})$.

- $v_{f}(\overline{1})=0$.

- $v_{f}(\bar{g}+\bar{h}) \geq \min \left\{v_{f}(\bar{g}), v_{f}(\bar{h})\right\}$, com igualdade se $v_{f}(\bar{g}) \neq v_{f}(\bar{h})$.

para todo $\bar{g}, \bar{h} \in \mathcal{O}_{f}$.

\subsection{Alguns resultados de álgebra linear}

Nesta seção apresentamos alguns resultados sobre quocientes e sequências exatas de espaços vetoriais.

Sejam $U, V$ e $W$ espaços vetoriais sobre um corpo $k$. Uma sequência é um diagrama da forma

$$
W \stackrel{\psi}{\longrightarrow} V \stackrel{\varphi}{\longrightarrow} U
$$

onde $\psi$ e $\varphi$ são homomorfismos de espaços vetoriais.

Definição 4.2.1 Diremos que uma sequência $W \stackrel{\psi}{\longrightarrow} V \stackrel{\varphi}{\longrightarrow} U$ é exata quando $\operatorname{Im}(\psi)=\operatorname{ker}(\varphi)$

Uma sequência exata da forma

$$
0 \stackrel{\Delta}{\longrightarrow} V \stackrel{\psi}{\longrightarrow} U
$$

implica que $\psi$ é injetora. Enquanto que uma sequência exata da forma

$$
W \stackrel{\varphi}{\longrightarrow} V \stackrel{\triangle}{\longrightarrow} 0
$$

implica que $\varphi$ é sobrejetora.

Seja $W \subset V$ um subespaço vetorial. Então

é um espaço vetorial.

$$
\frac{V}{W}=\{\bar{v}=v+W, v \in V\}
$$

Além disso, se $\operatorname{dim}_{k} V<\infty$, então

$$
\operatorname{dim}_{k} \frac{V}{W}=\operatorname{dim}_{k} V-\operatorname{dim}_{k} W
$$


Proposição 4.2.2 Consideremos a seguinte sequência exata de espaços vetoriais

$$
0 \longrightarrow W \stackrel{\psi}{\longrightarrow} V \stackrel{\varphi}{\longrightarrow} U \longrightarrow 0
$$

Então $V$ é de dimensão finita se, e somente se, $U$ e $W$ são de dimensão finita. Neste caso,

$$
\operatorname{dim}_{k} V=\operatorname{dim}_{k} W+\operatorname{dim}_{k} U
$$

\section{Demonstração:}

Como a sequência é exata, temos que $\operatorname{Im}(\varphi)=U$ e

$$
\operatorname{dim}_{K} W=\operatorname{dim}_{K} \operatorname{Im}(\psi)=\operatorname{dim}_{K} \operatorname{ker}(\varphi) \leq \operatorname{dim}_{K} V
$$

Então $\operatorname{dim}_{K} V$ é finita se, e somente se, $\operatorname{dim}_{K} U$ e $\operatorname{dim}_{K} W$ são finitas. Segue do Teorema do Isomorfismo que

$$
\frac{V}{\operatorname{ker}(\varphi)} \simeq U
$$

De 4.1 segue que

$$
\operatorname{dim}_{K} V=\operatorname{dim}_{K} \operatorname{ker}(\varphi)+\operatorname{dim}_{K} U=\operatorname{dim}_{K} W+\operatorname{dim}_{K} U
$$

Proposição 4.2.3 Sejam $W \subset V \subset U$ espaços vetoriais tais que $\operatorname{dim}_{k} \frac{U}{W}<\infty$. Então

$$
\operatorname{dim}_{k} \frac{U}{W}=\operatorname{dim}_{k} \frac{V}{W}+\operatorname{dim}_{k} \frac{U}{V}
$$

\section{Demonstração:}

Este resultado segue da proposição anterior usando a sequência exata:

$$
0 \longrightarrow \frac{V}{W} \longrightarrow \frac{U}{W} \longrightarrow \frac{U}{V} \longrightarrow 0
$$

Proposição 4.2.4 (Teorema do Isomorfismo de Noether) Sejam $V$ e.W subespaços de um espaço vetorial $U$. Então

$$
\frac{V}{V \cap W} \simeq \frac{V+W}{W}
$$

\section{Demonstração:}

A aplicação

$$
\begin{array}{ccc}
V+W & \longrightarrow & \frac{V}{V \cap_{\bar{v}} W} \\
v+w & \mapsto
\end{array}
$$

é injetora, sobrejetora e seu núcleo é $W$. 
Proposição 4.2.5 Sejam $V$ um espaço vetorial e $L: V \rightarrow V$ uma aplicação linear injetora. Seja $W$ um subespaço vetorial de $V$ tal que $L(W) \subset W$. Então

$$
\frac{V}{W} \simeq \frac{L(V)}{L(W)}
$$

Além disso, se $\frac{V}{W}$ e $\frac{V}{L(V)}$ são espaços de dimensão finita, então

$$
\operatorname{dim}_{k} \frac{W}{L(W)}=\operatorname{dim}_{k} \frac{V}{L(V)}
$$

\section{Demonstração:}

Consideremos a sequência,

$$
0 \longrightarrow W \stackrel{j}{\rightarrow} V \stackrel{\ell}{\rightarrow} \frac{L(V)}{L(W)} \longrightarrow 0
$$

onde $j$ é a inclusão e $\ell$ é o homomorfismo induzido por $L$.

Como $L$ é injetora, temos que

$$
\operatorname{ker}(\ell)=\{v \in V ; L(v) \in L(W)\}=W
$$

e a sequência acima é exata.

Consideremos agora as seguintes cadeias de inclusões

$$
W \subset W+L(V) \subset V
$$

e

$$
L(W) \subset W \cap L(V) \subset L(V) .
$$

Então $\operatorname{dim}_{K} \frac{L(V)}{L(W)}=\operatorname{dim}_{K} \frac{V}{W}<\infty$, e da Proposição 4.2 .4 temos

$$
\begin{aligned}
\operatorname{dim}_{K} \frac{V}{W} & =\operatorname{dim}_{K} \frac{W+L(V)}{W}+\operatorname{dim}_{K} \frac{V}{W+L(V)} \\
\| & \| \operatorname{dim}_{K} \frac{L(V)}{L(W)}=\operatorname{dim}_{K} \frac{L(V)}{W \cap L(V)}+\operatorname{dim}_{K} \frac{W \cap L(V)}{L(W)}
\end{aligned}
$$

onde a igualdade

$$
\operatorname{dim}_{K} \frac{W+L(V)}{W}=\operatorname{dim}_{K} \frac{L(V)}{W \cap L(V)}
$$

segue da Proposição 4.2.4.

Consequentemente, temos

$$
\operatorname{dim}_{K} \frac{V}{W+L(V)}=\operatorname{dim}_{K} \frac{W \cap L(V)}{L(W)}<\infty
$$

Por hipótese, $\operatorname{dim}_{K} \frac{V}{L(V)}<\infty$, da cadeia

$$
L(V) \subset W+L(V) \subset V
$$

temos

$$
\operatorname{dim}_{K} \frac{V}{L(V)}=\operatorname{dim}_{K} \frac{V}{W+L(V)}+\operatorname{dim}_{K} \frac{W+L(V)}{L(V)}
$$


Isto implica, usando a Proposição 4.2 .4 e a igualdade (4.2) que

$$
\operatorname{dim}_{K} \frac{W}{W \cap L(V)}=\operatorname{dim}_{K} \frac{W+L(V)}{L(V)}<\infty .
$$

Das equações (4.2), (4.3), e da cadeia acima que

$$
L(W) \subset W \cap L(V) \subset W .
$$

Segue que

$$
\operatorname{dim}_{K} \frac{W}{L(W)}=\operatorname{dim}_{K} \frac{W}{W \cap L(V)}+\operatorname{dim}_{K} \frac{W \cap L(V)}{L(W)}<\infty .
$$

$\mathrm{O}$ resultado segue agora da última igualdade e das equações (4.2) e (4.3).

\section{3 Índice de interseção}

Nesta seção vamos introduzir um método para expressar numericamente a ordem ou o grau de contato entre duas curvas algebróides planas. Isto será feito através da noção de índice de interseção.

Proposição 4.3.1 Sejam $f, g \in \mathcal{M}$. As seguinte condições são equivalentes

(i) $f$ e $g$ são relativamente primos.

(ii) A dimensão de $\frac{k[[X, Y]]}{\langle f, g\rangle}$ como espaço vetorial é finita.

\section{Demonstração:}

Como as condições (i) e (ii)são preservadas por automorfismos de $k[[X, Y]]$ e pela multiplicação de $f$ e $g$ por unidades de $k[[X, Y]]$, podemos assumir que $f$ e $g$ são pseudo-polinômios.

Suponhamos que $f$ e $g$ são relativamente primos em $k[[X, Y]]$. Portanto, são relativamente primos em $k[[X]][Y]$ e $R_{Y}(f, g) \neq 0$ (ver Teorema 2.4.3). Então $R_{Y}(f, g)=X^{r} u$, onde $r \geq 0$ e $u(0) \neq 0$. Segue da Proposição 2.4 .5 que $R_{Y}(f, g) \in\langle f, g\rangle$, logo $R_{Y}(f, g)(0)=0$ e como $u(0) \neq 0$, segue que $r \geq 1$. Portanto, $X^{r} \in\langle f, g\rangle$.

Suponhamos que $\partial_{Y} f=n$. Pelo Teorema da Divisão, dado $h \in k[[X, Y]]$, existem $q \in k[[X, Y]]$ e $a_{i}(X) \in k[[X]], i=0, \ldots, n-1$, tais que

$$
h=f q+a_{0}(X)+a_{1}(X) Y+\ldots+a_{n-1}(X) Y^{n-1} .
$$

Logo, a imagem $\bar{h}$ de $h$ em $\frac{k[[X, Y]]}{\langle f, g\rangle}$ está no $k$-espaço vetorial gerado por $\bar{X}^{i} \bar{Y}^{j}, 0 \leq i \leq r-1$, $0 \leq j \leq n-1$, pois $X^{r} \in\langle f, g\rangle$. Portanto, vale $(i i)$.

Suponhamos agora que $f$ e $g$ não são relativamente primos em $k[[X, Y]]$. Então existe $h \in k[[X]][Y]$ um pseudo-polinômio não invertível tal que $f=h f_{1}$ e $g=h g_{1}$.

Portanto, $\langle f, g\rangle \subseteq\langle h\rangle$. Afirmamos que $1, \bar{X}, \bar{X}^{2}, \ldots$ são linearmente independentes sobre $k$ em $\frac{k[[X, Y]]}{\langle h\rangle}$ pois, caso contrário, existiria um polinômio $P(X) \in k[X]$ tal que $P(X)=h_{1} h$, para algum $h_{1} \in k[[X, Y]]$, contradizendo o fato que $h$ é um pseudo-polinômio em $Y$.

Logo,

$$
\operatorname{dim}_{k} \frac{k[[X, Y]]}{\langle f, g\rangle} \geq \operatorname{dim}_{k} \frac{k[[X, Y]]}{\langle h\rangle}=\infty
$$


Definição 4.3.2 Sejam $f, g \in \mathcal{M}$. O indice de interseção de $f$ e g é o inteiro ( ou $\infty$ )

$$
I(f, g)=\operatorname{dim}_{k} \frac{k[[X, Y]]}{\langle f, g\rangle} .
$$

Vamos denotar por $\bar{z}$ a classe de $z \in k[[X, Y]]$ em $\mathcal{O}_{f}$. Então

$$
I(f, g)=\operatorname{dim}_{k} \frac{k[[X, Y]]}{\langle f, g\rangle}=\operatorname{dim}_{k} \frac{\frac{k[[X, Y]]}{\langle f\rangle}}{\langle\bar{g}\rangle}=\operatorname{dim}_{k} \frac{\mathcal{O}_{f}}{\langle\bar{g}\rangle} .
$$

Definição 4.3.3 Dizemos que duas curvas algebróides $(f)$ e $(g)$ são transversais se $(f)$ e $(g)$ são regulares e suas retas tangentes são distintas.

Teorema 4.3.4 Sejam $f, g \in \mathcal{M}, \Phi$ um automorfismo de $k[[X, Y]]$ e u e v unidades em $k[[X, Y]]$. $O$ índice de interseção possui as seguintes propriedades:

(i) $I(f, g)<\infty \Longleftrightarrow f$ e g são relativamente primos em $k[[X, Y]]$.

(ii) $I(f, g)=I(g, f)$.

(iii) $I(\Phi(f), \Phi(g))=I(u f, v g)=I(f, g)$.

(iv) $I(f, g h)=I(f, g)+I(f, h)$.

(v) $I(f, g)=1 \Longleftrightarrow(f) e(g)$ são transversais.

(vi) $I(f, g-h f)=I(f, g)$.

\section{Demonstração:}

(i) Proposição 4.3.1.

(ii) $I(f, g)=\operatorname{dim}_{k} \frac{k[[X, Y]]}{\langle f, g\rangle}=\operatorname{dim}_{k} \frac{k[[X, Y]]}{\langle g, f\rangle}=I(g, f)$, pois $\langle f, g\rangle=\langle g, f\rangle$.

(iii) A igualdade $I(u f, v g)=I(f, g)$ segue do fato que $\langle u f, v g\rangle=\langle f, g\rangle$.

Um automorfismo $\Phi$ induz um isomorfismo entre $\frac{k[[X, Y]]}{\langle\Phi(f), \Phi(g)\rangle}$ e $\frac{k[[X, Y]]}{\langle f, g\rangle}$. Logo,

$$
I(\Phi(f), \Phi(g))=\operatorname{dim}_{k} \frac{k[[X, Y]]}{\langle\Phi(f), \Phi(g)\rangle}=\operatorname{dim}_{k} \frac{k[[X, Y]]}{\langle f, g\rangle}=I(f, g) .
$$

(iv) Se $f$ e $g$ tem tator comum então $I(f, g)=I(f, g h)=\infty$. Suponhamos que $f$ e $g$ não tem fator comum. Vamos mostrar que a seguinte sequência de $k$-espaços vetoriais é exata

$$
0 \longrightarrow \frac{\mathcal{O}_{f}}{\langle\bar{h}\rangle} \stackrel{\psi}{\longrightarrow} \frac{\mathcal{O}_{f}}{\langle\bar{g} \bar{h}\rangle} \stackrel{\varphi}{\longrightarrow} \frac{\mathcal{O}_{f}}{\langle\bar{g}\rangle} \longrightarrow 0,
$$

onde $\varphi$ é induzida pela projeção $\tilde{\varphi}: \mathcal{O}_{f} \longrightarrow \frac{\mathcal{O}_{f}}{\langle\bar{g}\rangle}$ e aplicação $\psi$ é definida por

$$
\psi(\bar{z}+\langle\bar{h}\rangle)=\overline{g z}+\langle\bar{g} \bar{h}\rangle .
$$


Mostremos que $\psi$ é injetora. Suponhamos que $\psi(\bar{z}+\langle\bar{h}\rangle)=\bar{g} \bar{z}+\langle\bar{g} \bar{h}\rangle=0$. Isto implica que existe $\bar{\alpha} \in \mathcal{O}_{f}$ tal que $\bar{g} \bar{z}=\overline{\alpha g} \bar{h}$ donde $g z-\alpha g h \in\langle f\rangle$. Logo, existe $\beta \in k[[X, Y]]$, tal que $g z-\alpha g h=\beta f$. Como $f$ não divide $g$ então $f$ divide $z-\alpha h$. Portanto, $\overline{(z-\alpha h)}=0$, donde $\bar{z} \in\langle\bar{h}\rangle$. Logo, $\bar{z}+\langle\bar{h}\rangle=0$ e, portanto, $\psi$ é injetora.

Temos que $\operatorname{ker}(\varphi)=\frac{\langle\bar{g}\rangle}{\langle\bar{g} \bar{h}\rangle}$. Como, $\operatorname{Im}(\psi)=\frac{\langle\bar{g}\rangle}{\langle\bar{g} \bar{h}\rangle}$, a sequência

$$
0 \longrightarrow \frac{\mathcal{O}_{f}}{\langle\bar{h}\rangle} \stackrel{\psi}{\longrightarrow} \frac{\mathcal{O}_{f}}{\langle\bar{g} \bar{h}\rangle} \stackrel{\varphi}{\longrightarrow} \frac{\mathcal{O}_{f}}{\langle\bar{g}\rangle} \longrightarrow 0
$$

é exata. Logo, do Teorema 4.2.2

$$
\operatorname{dim}_{k} \frac{\mathcal{O}_{f}}{\langle\bar{g} \bar{h}\rangle}=\operatorname{dim}_{k} \frac{\mathcal{O}_{f}}{\langle\bar{h}\rangle}+\operatorname{dim}_{k} \frac{\mathcal{O}_{f}}{\langle\bar{g}\rangle}
$$

e o resultado segue.

(v) Suponhamos $(f)$ e $(g)$ transversais, ou seja, $(f)$ e $(g)$ são regulares com tangentes distintas. Após mudanças de coordenadas, se necessário, podemos assumir que

$$
f=X u+Y f_{1} \text { e } g=Y v+X g_{1}
$$

onde $u$ e $v$ unidades são e $f_{1}, g_{1} \in \mathcal{M}$. Então

$$
Y\left(v-u^{-1} f_{1} g_{1}\right)=Y v-u^{-1} Y f_{1} g_{1}=g-X g_{1}+u^{-1} g_{1}(X u-f)=g-\dot{u}^{-1} g_{1} f \in\langle f, g\rangle .
$$

Como $\left(v-u^{-1} f_{1} g_{1}\right)(0,0)=v(0,0) \neq 0$, segue que $v-u^{-1} f_{1} g_{1}$ é uma unidade e, portanto, $Y \in(f, g)$.

Do mesmo modo, temos que

$$
X\left(u-v^{-1} f_{1} g_{1}\right)=X u-v^{-1} X f_{1} g_{1}=f-Y f_{1}+v^{-1} f_{1}(Y v-g)=f-v^{-1} f_{1} g \in\langle f, g\rangle .
$$

Como $\left(u-v^{-1} f_{1} g_{1}\right)(0,0)=u(0,0) \neq 0$, segue que $u-v^{-1} f_{1} g_{1}$ é uma unidade e, portanto, $X \in\langle f, g\rangle$.

Assim, $\langle f, g\rangle=\langle X, Y\rangle \mathrm{e}$

$$
I(f, g)=\operatorname{dim}_{k} \frac{k[[X, Y]]}{\langle f, g\rangle}=\operatorname{dim}_{k} \frac{k[[X, Y]]}{\langle X, Y\rangle}=1 .
$$

Reciprocamente, suponhamos que mult $(f) \geq 2$, ou mult $(g) \geq 2$, ou $(f)$ e $(g)$ são regulares e possuem a mesma reta tangente. Após mudança linear de coordenadas podemos supor que $f=Y f_{1}+f_{2}$ e $g=Y g_{1}+g_{2}, \operatorname{com} f_{1}, g_{1} \in k[[X, Y]]$ e $f_{2}, g_{2} \in \mathcal{M}^{2}$. Logo, $\langle f, g\rangle \subset\langle Y\rangle+\mathcal{M}^{2}$ e

$$
\operatorname{dim}_{k} \frac{k[[X, Y]]}{\langle f, g\rangle} \geq \operatorname{dim}_{k} \frac{k[[X, Y]]}{\langle Y\rangle+\mathcal{M}^{2}}=\operatorname{dim}_{k} \frac{k[[X]]}{\left\langle X^{2}\right\rangle}=2
$$

(vi) Segue do fato que $\langle f, g-h f\rangle=\langle f, g\rangle$. 
Teorema 4.3.5 Seja $I^{r}: \mathcal{M} \times \mathcal{M} \longrightarrow \mathbb{N} \cup\{\infty\}$ uma aplicação satisfazendo as propriedades de (i) a (vi) do Teorema 4.3.4. Entĩo $I=I^{\prime}$.

\section{Demonstração:}

A prova será feita por indução sobre $I^{\prime}(f, g)$.

Podemos assumir que $f . g \in \mathcal{M} \backslash\{0\}$ são relativamente primos em $k[[X, Y]]$, pois caso contrário, de $(i)$ temos que $I^{\prime}(f, g)=I(f, g)=\infty$. Além disso, de $(v)$ segue que se $I^{\prime}(f, g)=1$ então $I(f, g)=1$.

Por hipótese de indução. suponhamos que se $I^{\prime}(f, g) \leq r-1$ então $I^{\prime}(f, g)=I(f, g)$.

Sejam $f, g \in \mathcal{M} \backslash\{0\}$ tais que $I^{\prime}(f, g)=r$. Seja $\phi$ um automorfismo de $k[[X, Y]]$ de modo que $\phi(f)$ e $\phi(g)$ são associados aos pseudo-polinômios mônicos $p$ e $q$. Da propriedade (iii) segue que

$$
I^{\prime}(f, g)=I^{\prime}(p, q) \text { e } I(f, g)=I(p, q) .
$$

Suponhamos que $\partial_{Y}(p)=n$ e $\partial_{Y}(q)=m$. Da propriedade (ii), podemos assumir, sem perda de generalidade, que $n \leq m$. Definimos $q_{1}=q-Y^{m-n} p$. Observemos que $q_{1}=X q_{2}$, para algum $q_{2} \in k[[X, Y]]$. Se $q_{2}$ é uma unidade, segue de $(i i i),(i v)$ e $(v i)$ que

$$
I^{\prime}(p, q)=I^{\prime}\left(p, q_{1}\right)=I^{\prime}\left(p, X q_{2}\right)=I^{\prime}(p, X)=I^{\prime}\left(Y^{n}, X\right)=n I^{\prime}(Y, X)=n=I(p, q) .
$$

Se $q_{2}$ não é uma unidade, segue de $(v i),(i v)$ e da hipótese de indução que

$$
r=I^{\prime}(p, q)=I^{\prime}\left(p, q_{1}\right)=I^{\prime}\left(p, X q_{2}\right)=I^{\prime}(p, X)+I^{\prime}\left(p, q_{2}\right)=I(p, X)+I\left(p, q_{2}\right)=I(p, q) .
$$

Logo, $I^{\prime}(f, g)=I(f, g)$.

O Teorema acima é um algoritmo executável para calcular $I(f, g)$ quando $f, g \in \mathcal{M} \backslash\{0\}$ são pseudo-polinômios.

Teorema 4.3.6 Sejam $f, g \in \mathcal{M} \backslash\{0\}$ pseudo-polinômios em $k[[X]][Y]$. Seja $f=f_{1} \ldots f_{r}$ a decomposição de $f$ em fatores irredutiveis, os quais podemos supor que são pseudo-polinômios. Então

$$
I(f, g)=\sum_{i=1}^{r} v_{f_{i}}\left(\bar{g}^{i}\right)=\text { mult }\left(R_{Y}(f, g)\right),
$$

onde $\bar{g}^{i}$ é a classe de $g$ em $\mathcal{O}_{f_{i}}$.

\section{Demonstração:}

Se $f$ e $g$ tem componente em comum, então

$$
I(f, g)=\infty=\operatorname{mult}\left(R_{Y}(f, g)\right) .
$$

Observemos que existe $i$ tal que $H_{\varphi_{i}}(\bar{g})=0$, exatamente $o i$ tal que $f_{i}$ é fator comum de $f$ e $g$, assim

$$
\sum_{i=1}^{r} v_{f_{i}}\left(\bar{g}^{i}\right)=\sum_{i=1}^{r} \operatorname{mult}\left(H_{\varphi_{i}}\left(\bar{g}^{i}\right)\right)=\infty,
$$

onde $\left(T^{n_{i}}, \varphi_{i}(T)\right)$ é a parametrização de $f_{i}$, que é um pseudo-polinômio irredutível. 
Suponhamos que $f$ e $g$ não tem componente em comum. Da propriedade $(i v)$, temos que $I(f, g)=$ $\sum_{i=1}^{r} I\left(f_{i}, g\right)$. Além disso,

$$
R_{Y}(f, g)=\prod_{i=1}^{r} R_{Y}\left(f_{i}, g\right) .
$$

Assim, é suficiente mostrar que se $f$ é irredutível então

$$
I(f, g)=v_{f}(\bar{g})=\operatorname{mult}\left(R_{Y}(f, g)\right) .
$$

Consideremos a aplicação $k$-linear

$$
\begin{array}{ccc}
L: k[[T]] & \longrightarrow & k[[T]] \\
h(T) & \longmapsto h(T) g\left(T^{n}, \varphi(T)\right)
\end{array}
$$

onde $n=\partial_{Y}(f)$ e $\left(T^{n}, \varphi(T)\right)$ é uma parametrização de Puiseux de $f$.

Como $f$ não é componente de $g$, então $g\left(T^{n}, \varphi(T)\right) \neq 0$ e, portanto, $L$ é injetora.

Seja $A_{\varphi}=k\left[\left[T^{n}, \varphi(T)\right]\right]\left(\cong \mathcal{O}_{f}\right)$ um $k$-subespaço vetorial de $k[[T]]$. Observemos que $L\left(A_{\varphi}\right) \subset A_{\varphi}$. Temos que

e

$$
\frac{A_{\varphi}}{L\left(A_{\varphi}\right)}=\frac{A_{\varphi}}{g\left(T^{n}, \varphi(T)\right) A_{\varphi}} \cong \frac{\mathcal{O}_{f}}{\langle\bar{g}\rangle}
$$

$$
\frac{k[[T]]}{L(k[[T]])}=\frac{k[[T]]}{\left\langle g\left(T^{n}, \varphi(T)\right\rangle\right.}
$$

Do Corolário 4.1.5, temos que $\operatorname{dim}_{k} \frac{k[T]]}{A_{\varphi}}<\infty$. Como

$$
\operatorname{dim}_{k} \frac{k[[T]]}{\left\langle g\left(T^{n}, \varphi(T)\right\rangle\right.}=\operatorname{mult}\left(g\left(T^{n}, \varphi(T)\right)<\infty\right.
$$

e $L\left(A_{\varphi}\right) \subset A_{\varphi}$, segue da Proposição 4.2 .5 que

$$
\operatorname{dim}_{k} \frac{A_{\varphi}}{L\left(A_{\varphi}\right)}=\operatorname{dim}_{k} \frac{k[[T]]}{L(k[[T]])}=\operatorname{mult}\left(g\left(T^{n}, \varphi(T)\right)=v_{f}(\bar{g})\right.
$$

Portanto,

$$
I(f, g)=\operatorname{dim}_{k} \frac{\mathcal{O}_{f}}{\langle\bar{g}\rangle}=\operatorname{dim}_{k} \frac{W}{L(W)}=v_{f}(\bar{g}) .
$$

Por outro lado, se $\xi$ é uma raiz $n$-ésima primitiva da unidade então $\varphi\left(\xi^{i} X^{\frac{1}{n}}\right), i=1, \ldots, n$, são as raízes de $f$ como polinômio em $Y$, e segue da Proposição 2.4.7 que

$$
R_{Y}(f, g)=\prod_{i=1}^{n} g\left(X, \varphi\left(\xi^{i} X^{\frac{1}{n}}\right)\right)
$$

Logo,

$$
\operatorname{mult}\left(R_{Y}(f, g)\right)=n \operatorname{mult}_{X^{\frac{1}{n}}} g\left(X, \varphi\left(X^{\frac{1}{n}}\right)\right)=\operatorname{mult}_{T}\left(g\left(T^{n}, \varphi(T)\right)=v_{f}(\bar{g})\right.
$$

Teorema 4.3.7 Sejam $f, g \in \mathcal{M}$. Então

$$
I(f, g) \geq \operatorname{mult}(f) \text { mult }(g)
$$

com igualdade se, e somente se, $(f)$ e $(g)$ não possuem tangentes comuns. 


\section{Demonstração:}

Sejam $f=f_{1} \ldots f_{r}$ e $g=g_{1} \ldots g_{s}$ as decomposiçōes de $f$ e $g$ em fatores irredutíveis. Das propriedades $(i i)$ e $(i v)$ temos que

$$
I(f, g)=\sum_{i, j} I\left(f_{i}, g_{j}\right)
$$

Por outro lado,

$$
\text { mult }(f) \text { mult }(g)=\sum_{i} \operatorname{mult}\left(f_{i}\right) \text { mult }(g)=\sum_{i, j} \operatorname{mult}\left(f_{i}\right) \text { mult }\left(g_{j}\right) .
$$

Das igualdades acima podemos reduzir o problema ao caso de $f$ e $g$ irredutíveis.

Suponhamos que, através de uma mudança de coordenadas, $f$ e $g$ são associados à pseudopolinômios em $k[[X]][Y]$ e que $(f)$ tem $(Y)$ como reta tangente. Seja $\left(T^{n}{ }_{r} \varphi(T)\right)$, onde $n=$ mult $(f)$, uma parametrização de Puiseux de $(f)$. Como $(Y)$ é a reta tangente de $f$, segue que mult $(\varphi(T))>n$.

Suponhamos que

$$
g(X, Y)=(a X+b Y)^{m}+g_{m+1}(X, Y)+\ldots
$$

Então

$$
I(f, g)=v_{f}(g)=\operatorname{mult}\left(g\left(T^{n}, \varphi(T)\right)=\operatorname{mult}\left(a T^{n}+b \varphi(T)\right)^{m}+g_{m+1}\left(T^{n}, \varphi(T)\right)+\ldots\right) \geq n m,
$$

com igualdade se, e somente se, $a \neq 0$, isto é, $(Y)$ não é reta tangente de $(g)$, ou equivalentemente as retas tangentes de $(f)$ e $(g)$ são distintas.

Observamos que o índice de interseção é efetivamente calculável pelos Teoremas 4.3.5 e 4.3.6, quando as duas curvas são dadas pela equação na forma cartesiana ou uma delas é dada na forma cartesiana e a outra na forma paramétrica. 


\section{Capítulo 5}

\section{Resolução de singularidades}

Neste capítulo vamos estudar o processo de resolução de singularidades de curvas irredutíveis planas que consiste em transformar as singularidades de uma curva em singularidades mais simples, por meio de uma sequência finita de certas transformações quadráticas que chamaremos de blowingups. Também exibiremos algumas propriedades e relações entre as curvas e seus blowing-ups.

\subsection{Transformações quadráticas em $\mathbb{C}^{2}$}

Definição 5.1.1 Uma transformação quadrática ou um blowing-up centrado na origem de $\mathbb{C}^{2}$ é uma aplicação que, em algum sistema de coordenadas em $\mathbb{C}^{2}$, é da forma:

$$
T: \begin{array}{cl}
\mathbb{C}^{2} & \longrightarrow \mathbb{C}^{2} \\
\left(X_{1}, Y_{1}\right) & \longmapsto(X, Y)=\left(X_{1}, X_{1} Y_{1}\right)
\end{array}
$$

A reta $E: X_{1}=0$ é chamada de divisor excepcional do blowing-up e é igual a $T^{-1}(0,0)$.

Seja $L: Y-a X=0$ uma reta em $\mathbb{C}^{2}$ que passa pela origem. Então $T^{-1}(L)$ é a união do divisor excepcional $E$ e da reta $Y_{1}=a$, que corta $E$ no ponto $(0, a)$. Portanto, os pontos do divisor excepcional correspondem à todas as direções de $\mathbb{C}^{2}$ que passam pela origem, exceto a direção do eixo $Y$.

A transformação $T$ induz um automorfismo analítico de $\mathbb{C}^{2} \backslash E$.

Seja $C_{f}$ uma curva analítica definida por $f \in \mathcal{M} \subset \mathbb{C}\{X, Y\}$. Vejamos quem é o conjunto $T^{-1}\left(C_{f}\right)$. Suponhamos que a expansão de $f$ em polinômios homogêneos é dada por

$$
f(X, Y)=F_{n}(X, Y)+F_{n+1}(X, Y)+\ldots
$$

Então $T^{-1}\left(C_{f}\right)$ é dada por

$$
f\left(T\left(X_{1}, Y_{1}\right)\right):=F_{n}\left(X_{1}, X_{1} Y_{1}\right)+F_{n+1}\left(X_{1}, X_{1} Y_{1}\right)+\ldots=X_{1}^{n}\left(F_{n}\left(1, Y_{1}\right)+X_{1} F_{n+1}\left(1, Y_{1}\right)+\ldots\right)=0 .
$$

Chamamos $f\left(T\left(X_{1}, Y_{1}\right)\right)$ de transformada total de $f$ e a curva associada $T^{-1}\left(C_{f}\right)$ será chamada de transformada total de $C_{f}$.

A curva $C_{f^{(1)}}$, determinada pela equação $f^{(1)}\left(X_{1}, Y_{1}\right)=0$, onde

$$
f^{(1)}\left(X_{1}, Y_{1}\right)=F_{n}\left(1, Y_{1}\right)+X_{1} F_{n+1}\left(1, Y_{1}\right)+\ldots+X_{1}^{j-n} F_{j}\left(1, Y_{1}\right)+\ldots
$$

é chamada de transformada estrita de $C_{f}$. Como $T^{-1}(0,0)$ é o divisor excepcional, com equação $E: X_{1}=0$, temos que $T^{-1}\left(C_{f}\right)=E \cup C_{f^{(1)}}$. 
Exemplo 5.1.2 Consideremos a curva $C_{f}$ onde $f: Y^{2}-a^{2} X^{2}-X^{3}=0$, com $a \neq 0$. Como a curva é dada por um polinômio não teremos problemas de convergência e, portanto, $f$ está definida em todo $\mathbb{C}^{2}$.

A transformada total de $C_{f}$ é

$$
T^{-1}\left(C_{f}\right): f\left(T\left(X_{1}, Y_{1}\right)\right)=f\left(X_{1}, X_{1} Y_{1}\right)=X_{1}^{2} Y_{1}^{2}-a^{2} X_{1}^{2}-X_{1}^{3}=X_{1}^{2}\left(Y_{1}^{2}-a^{2}-X_{1}\right)=0
$$

enquanto que a transformada estrita de $C_{f}$ é dada por

$$
C_{f(1)}: Y_{1}^{2}-a^{2}-X_{1}=0
$$

Observemos que as retas tangentes à $C_{f}$ na origem são dadas por

$$
Y^{2}-a^{2} X^{2}=(Y-a X)(Y+a X)=0
$$

que têm por transformadas estritas $Y_{1}=a$ e $Y_{1}=-a$, que são retas horizontais passando pelos pontos $P_{1}=(0, a)$ e $P_{2}=(0,-a)$, respectivamente. Estes pontos são as interseções de $C_{f^{(1)}}$ com $E$.
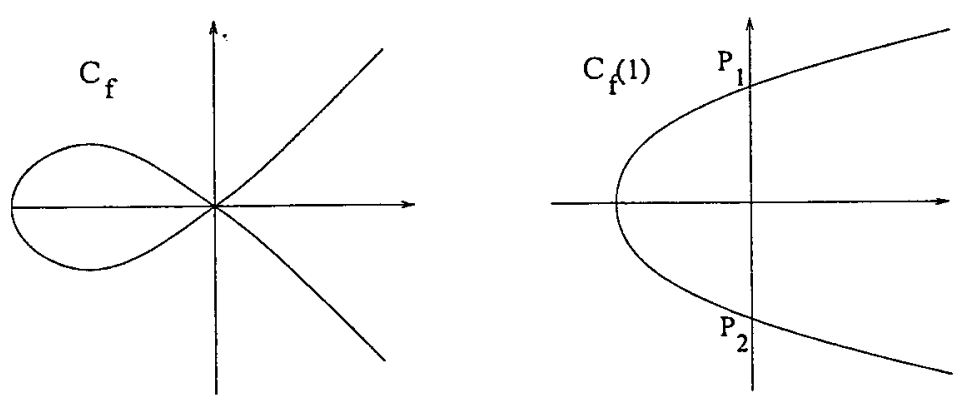

Figura 5.1: Seqüência de blowing-ups (cúbica nodal)

Observemos que após um blowing-up a curva $C_{f}$ é transformada em uma curva suave $C_{f^{(1)}}$ e seus dois ramos que passam pela origem são transformadas em ramos da curva que passam por $P_{1}$ e $P_{2}$.

Exemplo 5.1.3 Consideremos a curva $C_{f}$ onde $f: Y^{2}-X^{3}=0$. Como a curva é dada por um polinômio não teremos problemas de convergência e, portanto, $f$ está definida em todo $\mathbb{C}^{2}$.

A transformada total de $C_{f}$ é

$$
T^{-1}\left(C_{f}\right): f\left(T\left(X_{1}, Y_{1}\right)\right)=f\left(X_{1}, X_{1} Y_{1}\right)=X_{1}^{2} Y_{1}^{2}-X_{1}^{3}=X_{1}^{2}\left(Y_{1}^{2}-X_{1}\right)=0
$$

enquanto que a transformada estrita de $C_{f}$ é dada por

$$
C_{f^{(1)}}: Y_{1}^{2}-X_{1}=0
$$

que tem como reta tangente ná origem $X_{1}=0$. Notemos que as retas tangentes à $C_{f}$ na origem são dadas por $Y=0$ que têm por transformadas estritas $Y_{1}=0$. 

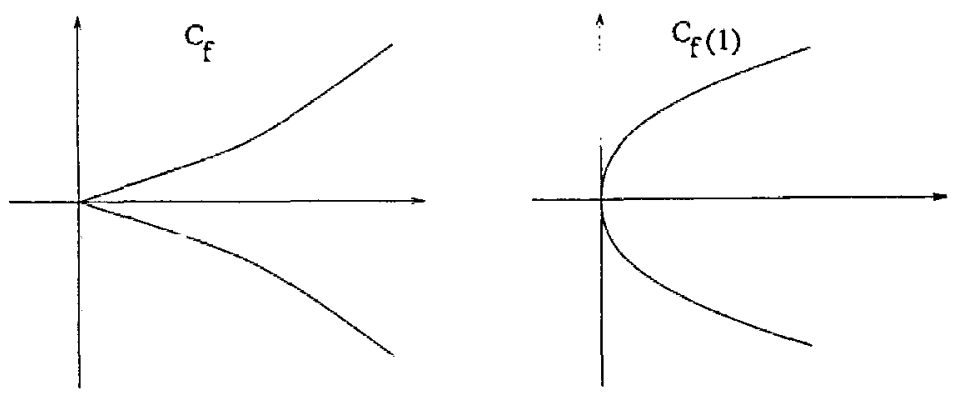

Figura 5.2: Seqüência de blowing-ups (cúspide)

A curva $C_{f}$ é uma cúspide. Após um blowing-up a curva $C_{f}$ é transformada em uma parábola, que é a $C_{f(1)}$. Como $C_{f}$ tem um único ramo passando pela origem, este é transformado no único ramo de $C_{f(1)}$ que passa pela origem.

Exemplo 5.1.4 Consideremos a curva $C_{f}$ onde $f: Y^{2}-X^{5}=0$.

A transformada total de $C_{f}$ é

$$
T^{-1}\left(C_{f}\right): f\left(T\left(X_{1}, Y_{1}\right)\right)=f\left(X_{1}, X_{1} Y_{1}\right)=X_{1}^{2} Y_{1}^{2}-X_{1}^{5}=X_{1}^{2}\left(Y_{1}^{2}-X_{1}^{3}\right)=0
$$

enquanto que a transformada estrita de $C_{f}$ é dada por

$$
C_{f^{(1)}}: Y_{1}^{2}-X_{1}^{3}=0
$$

que tem como reta tangente na origem $Y_{1}=0$. Notemos que $C_{f^{(1)}}$ é a curva do exemplo anterior e, portanto, com mais um blowing-up transformamos esta curva na parábola $Y_{2}^{2}-X_{2}=0$.
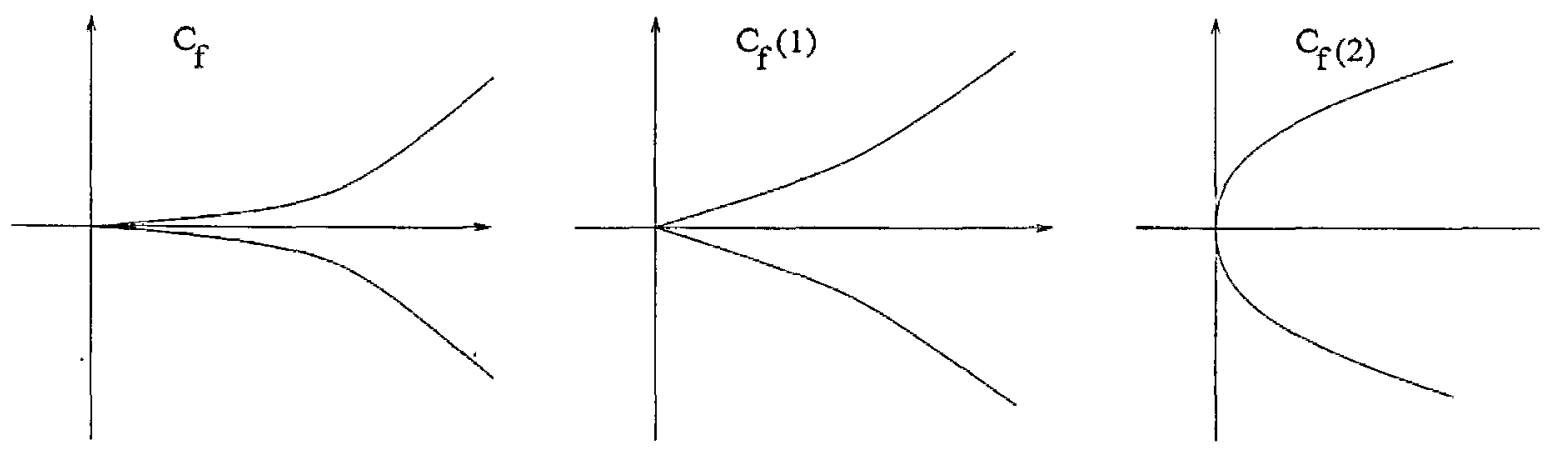

Figura 5.3: Sequiência de blowing-ups (cúspide de ordem 2)

Alguns dos fatos apontados nos exemplos anteriores sempre ocorrem. Por exemplo, vamos mostrar que após um número finito de blowing-ups é possível transformar qualquer curva singular em uma curva regular. Este procedimento será chamado de resolução de singularidades de umạ curva e permitirá o estudo de uma singularidade através da sua transformação em uma singularidade mais simples. 


\subsection{Resolução de singularidades de curvas planas}

A partir desta seção $k$ será um corpo algebricamente fechado qualquer.

Definição 5.2.1 Uma transformação quadrática $\sigma$ do anel $k[[X, Y]]$ no anel $k\left[\left[X_{1}, Y_{1}\right]\right]$ é um homomorfismo de k-álgebras, definido por

$$
\begin{aligned}
& \sigma: k[[X, Y]] \longrightarrow k\left[\left[X_{1}, Y_{1}\right]\right] \\
& X \quad \longmapsto \quad X_{1} \\
& Y \quad \longmapsto \quad X_{1} Y_{1}
\end{aligned}
$$

ou por

$$
\begin{aligned}
& \tau: k[[X, Y]] \longrightarrow k\left[\left[X_{1}, Y_{1}\right]\right] \\
& \begin{array}{ccc}
X & \longmapsto & X_{1} Y_{1} \\
Y & \longmapsto & Y_{1}
\end{array}
\end{aligned}
$$

Estas tranformações não são invertiveis, pois $X_{1} Y_{1}$ e $X_{1}$ (ou $X_{1} Y_{1}$ e $Y_{1}$ ) são linearmente dependentes, mas são birracionais, isto é, elas definem isomorfismos entre $k((X, Y))$ e $k\left(\left(X_{1}, Y_{1}\right)\right)$, respectivamente os corpos de frações de $k[[X, Y]]$ e $k\left[\left[X_{1}, Y_{1}\right]\right]$.

O homomorfismo $\sigma$ leva o ideal $\langle X, Y\rangle$ no ideal $\left\langle X_{1}, X_{1} Y_{1}\right\rangle=\left\langle X_{1}\right\rangle$.

No anel $\mathbb{C}\{X, Y\}$, esța condição corresponde geometricamente à condição $T^{-1}(0,0)=E$ da seção anterior, onde

$$
T: \begin{array}{cl}
\mathbb{C}^{2} & \longrightarrow \mathbb{C}^{2} \\
\left(X_{1}, Y_{1}\right) & \longmapsto(X, Y)=\left(X_{1}, X_{1} Y_{1}\right)
\end{array}
$$

e $E: X_{1}=0$ é o divisor excepcional do blowing-up $T$.

A transformação por $\sigma$ de

$$
f(X, Y)=F_{n}(X, Y)+F_{n+1}(X, Y)+\ldots \in k[[X, Y]]
$$

é o elemento de $k\left[\left[X_{1}, Y_{1}\right]\right]$ :

$$
\begin{gathered}
\left.\sigma(f)=f\left(X_{1}, X_{1} Y_{1}\right)\right)=F_{n}\left(X_{1}, X_{1} Y_{1}\right)+F_{n+1}\left(X_{1}, X_{1} Y_{1}\right)+\ldots= \\
=X_{1}^{n}\left(F_{n}\left(1, Y_{1}\right)+X_{1} F_{n+1}\left(1, Y_{1}\right)+\ldots\right)=0 .
\end{gathered}
$$

A série

$$
\sigma^{*}(f)=\frac{1}{X_{1}^{n}} \sigma(f)=F_{n}\left(1, Y_{1}\right)+X_{1} F_{n+1}\left(1, Y_{1}\right)+\ldots
$$

onde $n=$ mult $(f)$, será chamada de transformada estrita de $(f)$ por $\sigma$, e será também denotada por $f^{(1)}$.

Propusıção 5.2.2 Sejam $f, g \in k[[X, Y]]$.

(i) $\sigma^{*}(f)$ é invertivel em $k\left[\left[X_{1}, Y_{1}\right]\right]$ se, e somente se, $f$ é regular em $X$, isto é, a forma inicial de $f$ é da forma $F_{n}=\left(c X^{n}+{ }^{\circ} ..\right)$, onde $c \in k \backslash\{0\}$ e n é um inteiro não negativo (o caso $n=0$ corresponde a $f$ invertivel em $k[[X, Y]])$.

(ii) $\sigma^{*}(f g)=\sigma^{*}(f) \sigma^{*}(g)$.

(iii) mult $\left(\sigma^{*}(f)\right) \leq$ mult $(f)$. 
(iv) Se $f$ é um polinômio de Weierstrass em $k[[X]][Y]$ de grau n com-cone tangente $\left(Y^{n}\right)$ então $\sigma^{*}(f)$ é um pseudo-polinômio de grau $n$ em $Y_{1}$.

(v) Se $f$ é irredutível então $\sigma^{*}(f)$ é irredutivel ou é uma unidade.

\section{Demonstração:}

Se $f(X, Y)=F_{n}(X, Y)+F_{n+1}(X, Y)+\ldots$ então

$$
\sigma^{*}(f)=F_{n}\left(1, Y_{1}\right)+X_{1} F_{n+1}\left(1, Y_{1}\right)+\ldots
$$

(i) A transformada estrita $\sigma^{*}(f)$ é uma unidade se, e somente, se $F_{n}(1,0)$ é uma constante não nula. Mas isto é equivalente a dizer que $F_{n}(X, Y)=\left(c X^{n}+\ldots\right)$, onde $c \in k \backslash\{0\}$.

(ii) Seja $g(X, Y)=G_{m}(X, Y)+G_{m+1}(X, Y)+\ldots$, então $\sigma^{*}(g)=G_{m}\left(1, Y_{1}\right)+X_{1} G_{m+1}\left(1, Y_{1}\right)+\ldots$. Temos que

$$
\begin{gathered}
\sigma^{*}(f g)=F_{n} G_{m}\left(1, Y_{1}\right)+X_{1}\left(F_{n} G_{m+1}\left(1, Y_{1}\right)+F_{n+1} G_{m}\left(1, Y_{1}\right)\right)+ \\
+X_{1}^{2}\left(F_{n} G_{m+2}\left(1, Y_{1}\right)+F_{n+1} G_{m+1}\left(1, Y_{1}\right)+F_{n+2} G_{m}\left(1, Y_{1}\right)\right)+\ldots=\sigma^{*}(f) \sigma^{*}(g) .
\end{gathered}
$$

(iii) Segue do fato que em $F_{n}\left(1, Y_{1}\right)$ temos monômios de grau menor ou igual a $n=$ mult $(f)$.

(iv) Seja $f=Y^{n}+a_{1}(X) Y^{n-1}+\ldots+a_{n}(X)$ um polinômio de Weierstrass com cone tangente $\left(Y^{n}\right)$, isto é, mult $\left(a_{i}(X)\right)>i, \forall i=1, \ldots, n$. Assim,

$$
\begin{gathered}
\sigma^{*}(f)=\frac{1}{X_{1}^{n}} f\left(X_{1}, X_{1} Y_{1}\right)=\frac{X_{1}^{n} Y_{1}^{n}}{X_{1}^{n}}+\frac{a_{1}\left(X_{1}\right) X_{1}^{n-1} Y_{1}^{n-1}}{X_{1}^{n}}+\ldots+\frac{a_{n}\left(X_{1}\right)}{X_{1}^{n}} \\
\sigma^{*}(f)=Y_{1}^{n}+\frac{a_{1}\left(X_{1}\right)}{X_{1}} Y_{1}^{n-1}+\ldots+\frac{a_{n}\left(X_{1}\right)}{X_{1}^{n}}
\end{gathered}
$$

com mult $\left(\frac{a_{i}\left(X_{1}\right)}{X_{1}^{i}}\right)>0$. Portanto, $\sigma^{*}(f)$ é um pseudo-polinômio de grau $n$ em $Y_{1}$.

(v) Como $f$ é irredutível, segue do Lema da Unitangente 3.3.6 que $f$ é regular em $X$ ou $f$ é regular em $Y$. Quando $f$ é regular em $Y$ mas não é regular em $X$, o cone tangente de $f$ é da forma $\left(Y^{n}\right)$.

Se $f$ é regular em $X$ então $\sigma^{*}(f)$ é uma unidade, pelo ítem (i). Então é suficiente mostrar que o resultado vale para $f$ irredutivel com cone tangente $\left(Y^{n}\right)$. Suponhamos $\sigma^{*}(f)$ redutível.

De $(i)$ e $(i i)$ temos que, se $f$ e $g$ são associados, $f=u g$ com $u$ uma unidade em $k[[X, Y]]$, então $\sigma^{*}(f)$ e $\sigma^{*}(g)$ são associados, pois $\sigma^{*}(f)=\sigma^{*}(u) \sigma^{*}(g)$ e $\sigma^{*}(u)$ é uma unidade. Portanto, podemos assumir que $f$ é um pseudo-polinômio de grau $n$ em $Y$. De $(i v)$ segue que $\sigma^{*}(f)$ é um pseudo-polinômio de grau $n$ em $Y_{1}$. Como $\sigma^{*}(f)$ é redutível em $k\left[\left[X_{1}, Y_{1}\right]\right]$, segue do Lema $2.2 .2 \mathrm{e}$ do Lema 2.2.3 que ele pode ser fatorado em um produto de pseudo-polinômios irredutíveis. Seja $h\left(X_{1}, Y_{1}\right)$ um pseudo-polinômio irredutível de grau $r, 0<r<n$, que divide $\sigma^{*}(f)$. Portanto, $X^{r} h\left(X, \frac{Y}{X}\right)$ deve ser um fator não trivial de $f$, o que é uma contradição, pois $f$ é irredutível. 
Observemos que se $f \in k[[X, Y]]$ é irredutível e $\sigma^{*}(f)$ não é uma unidade, então $f$ é regular em $Y$ com cone tangente $\left(Y^{n}\right)$, isto é, $f$ pode ser escrito como

$$
f=a_{0}(X) Y^{n}+a_{1}(X) Y^{n-1}+\ldots+a_{n}(X)+Y^{n+1} h(X, Y),
$$

com $a_{i}(X) \in k[[X]]$, mult $\left(a_{i}(X)\right)>i, \forall i=1, \ldots, n, a_{0}(0) \neq 0$ e $h \in k[[X, Y]]$.

Proposição 5.2.3 Seja $f \in k[[X, Y]]$ uma série de potêncids irredutivel com cone tangente $\left(Y^{n}\right)$. Suponhamos que $I(f, Y)=m$. Então

(i) $I\left(\sigma^{*}(f), Y_{1}\right)=m-n$ e $I\left(\sigma^{*}(f), X_{1}\right)=n$.

(ii) Se $m-n \geq n$, então mult $\left(\sigma^{*}(f)\right)=$ mult $(f)=n$. Além disso, se $m-n>n$ então $\left(\sigma^{*}(f)\right)$ tem cone tangente $\left(Y_{1}^{n}\right)$ e se $m-n=n$ então nem $\left(X_{1}\right)$ nem $\left(Y_{1}\right)$ são retas tangentes de $\left(\sigma^{*}(f)\right)$.

(iii) Se $m-n<n$, então mult $\left(\sigma^{*}(f)\right)=m-n<m u l t(f)$ e $\left(\sigma^{*}(f)\right)$ tem cone tangente $\left(X_{1}^{m-n}\right)$.

\section{Demonstração:}

Como $f$ é regular em $Y$ de ordem $n$, podemos escrever $f$ como segue

$$
f=a_{0}(X) Y^{n}+a_{1}(X) Y^{n-1}+\ldots+a_{n}(X)+Y^{n+1} h(X, Y),
$$

coin $a_{0}(0) \neq 0, h \in k[[X, Y]]$. Então $m=I(f, Y)=I\left(a_{n}(X), Y\right)=\operatorname{mult}\left(a_{n}(X)\right)$, pois $a_{n}(X)$ e $Y$ não possuem tangentes em comum (ver Teorema 4.3.7).

Do Lema 3.4.1, temos que

$$
\text { mult }\left(a_{i}(X)\right) \geq i \frac{\operatorname{mult}\left(a_{n}(X)\right)}{n}=i \frac{m}{n}
$$

Ainda,

$$
\begin{gathered}
\sigma^{*}(f)=\frac{a_{0}\left(X_{1}\right) X_{1}^{n} Y_{1}^{n}}{X_{1}^{n}}+\frac{a_{1}\left(X_{1}\right) X_{1}^{n-1} Y_{1}^{n-1}}{X_{1}^{n}}+\ldots+\frac{a_{n}\left(X_{1}\right)}{X_{1}^{n}}+\frac{Y_{1}^{n+1} X_{1}^{n+1} h\left(X_{1}, X_{1} Y_{1}\right)}{X_{1}^{n}} \\
=a_{0}\left(X_{1}\right) Y_{1}^{n}+\frac{a_{1}\left(X_{1}\right)}{X_{1}} Y_{1}^{n-1}+\ldots+\frac{a_{n}\left(X_{1}\right)}{X_{1}^{n}}+Y_{1}^{n+1} X_{1} h\left(X_{1}, X_{1} Y_{1}\right) \\
=b_{0}\left(X_{1}\right) Y_{1}^{n}+b_{1}\left(X_{1}\right) Y_{1}^{n-1}+\ldots+b_{n}\left(X_{1}\right)+Y_{1}^{n+1} X_{1} h\left(X_{1}, X_{1} Y_{1}\right),
\end{gathered}
$$

onde $b_{i}\left(X_{1}\right)=\frac{a_{i}\left(X_{1}\right)}{X_{1}^{2}}$. Então, $b_{0}\left(X_{1}\right)=a_{0}\left(X_{1}\right) \mathrm{e}$

$$
\text { mult }\left(b_{i}\left(X_{1}\right)\right)=\operatorname{mult}\left(\frac{a_{i}\left(X_{1}\right)}{X_{1}^{i}}\right)=\operatorname{mult}\left(a_{i}\left(X_{1}\right)\right)-i \geq i \frac{m}{n}-i=\frac{i(m-n)}{n} .
$$

(i) Como o cone tangente de $(f)$ é $\left(Y^{n}\right)$ temos que $m=$ mult $\left(a_{n}(X)\right)>n$. Ainda, $b_{0}\left(X_{1}\right)=a_{0}\left(X_{1}\right)$ é uma unidade e $\frac{i(m-n)}{n}>0, \forall i \geq 1$. Logo,

$$
I\left(\sigma^{*}(f), X_{1}\right)=I\left(b_{0}\left(X_{1}\right) Y_{1}^{n}, X_{1}\right)=I\left(Y_{1}^{n}, X_{1}\right)=n
$$

e

$$
I\left(\sigma^{*}(f), Y_{1}\right)=I\left(b_{n}\left(X_{1}\right), Y_{1}\right)=\operatorname{mult}\left(b_{n}\left(X_{1}\right)\right) \text { mult }\left(Y_{1}\right)=\operatorname{mult}\left(a_{n}\left(X_{1}\right)\right)-n=m-n
$$


(ii) Se $m-n \geq n$ então

$$
\text { mult }\left(b_{i}\left(X_{1}\right)\right) \geq \frac{i(m-n)}{n} \geq i
$$

Como $b_{0}(0) \neq 0$, temos que

$$
\operatorname{mult}\left(\sigma^{*}(f)\right)=n=\operatorname{mult}(f) .
$$

Se $m-n>n$ então mult $\left(b_{i}\left(X_{1}\right)\right)>i$, o que mostra que $\left(Y_{1}^{n}\right)$ é o cone tangente de $\left(\sigma^{*}(f)\right)$.

Se $m-n=n$ então mult $\left(b_{n}\left(X_{1}\right)\right)=n$ e assim o cone tangente de $\left(\sigma^{*}(f)\right)$ tem contribuições de $b_{0}\left(X_{1}\right) Y_{1}^{n}$ e $b_{n}\left(X_{1}\right)$. Isto mostra que nem $\left(X_{1}\right)$ nem $\left(Y_{1}\right)$ são retas tangentes de $\left(\sigma^{*}(f)\right)$.

(iii) Suponhamos que $0<m-n<n$, ou seja, $n<m<2 n$. Como

$$
\operatorname{mult}\left(b_{i}\left(X_{1}\right) Y_{1}^{n-i}\right) \geq i \frac{m}{n}-i+n-i>i-i+n-i=n-i .
$$

Logo, mult $\left(\sigma^{*}(f)\right)=m-n<n=$ mult $(f)$ e $\left(X_{1}^{m-n}\right)$ é o cone tangente de $\left(\sigma^{*}(f)\right)$.

Analogamente, podemos definir a noção de transformada estrita $\tau^{*}(f)$ e provar resultados análogos, invertendo as regras para $X$ e $Y$.

Lema 5.2.4 Seja $f \in k[[X, Y]]$ uma série de potências irredutivel de multiplicidade n e regular em $Y$. Então existe um automorfismo $\Phi$ de $k[[X, Y]]$ tal que $\Phi(f)$ é irredutivel de multiplicidade $n$, regular em $Y$ e tal que $I(\Phi(f), Y)$ não é um múltiplo de $n$.

\section{Demonstração:}

Como $f$ é irredutível e regular em $Y$ de ordem $n$, podemos escrever

$$
f=a_{0}(X) Y^{n}+a_{1}(X) Y^{n-1}+\ldots+a_{n}(X)+Y^{n+1} h(X, Y)
$$

com $a_{0}(0) \neq 0, h \in k[[X, Y]]$ e $I(f, Y)=\operatorname{mult}\left(a_{n}(X)\right)$.

Do Lema 3.4.1 temos que

$$
\operatorname{mult}\left(a_{i}(X)\right) \geq i \frac{\operatorname{mult}\left(a_{n}(X)\right)}{n}=i \frac{I(f, Y)}{n}
$$

Se $n$ não divide $I(f, Y)$, tomamos ${ }^{`} \Phi=I d$.

Suponhamos que $I(f, Y)=n r$, para algum inteiro $r \geq 1$. Sejam $c \in k$ e

$$
\Phi_{1}(X, Y)=\left(X, Y+c X^{r}\right) .
$$

Temos que

$$
\Phi_{1}(f(X, Y))=f\left(X, Y+c X^{r}\right)=a_{0}(X)\left(Y+c X^{r}\right)^{n}+\ldots+a_{n}(X)+\left(Y+c X^{r}\right)^{n+1} h\left(X, Y+c X^{r}\right) .
$$

Como

$$
\text { mult }\left(a_{i}(X)\right) \geq i \frac{I(f, Y)}{n}=i \frac{n r}{n}=i r
$$

podemos escrever

$$
\Phi_{1}(f(X, Y))=b_{0}(c, X) Y^{n}+b_{1}(c, X) Y^{n-1}+\ldots+b_{n}(c, X)+P(c) X^{n r}+Y^{n+1} h^{\prime}(X, Y),
$$


$\operatorname{com} b_{0}(c, 0)=a_{0}(0) \neq 0$

$$
\text { mult }\left(b_{i}(c, X)\right) \begin{cases}\geq i r, & \text { se } 1 \leq i \leq n-1 \\ >n r, & \text { se } i=n,\end{cases}
$$

e $P(c)$ é um polinômio de grau $n$ em $c$.

Tomando $c$ uma raiz de $P$, temos que

$$
\Phi_{1}(f(X, Y))=b_{0}(X) Y^{n}+b_{1}(X) Y^{n-1}+\ldots+b_{n}(X)+Y^{n+1} h^{\prime \prime}(X, Y) .
$$

Logo, $\Phi_{1}(f)$ é irredutível; regular em $Y$ e mult $\left(b_{n}(X)\right)>n r$.

Como $I\left(\Phi_{1}(f), Y\right)=$ mult $\left(b_{n}(X)\right)$ se $n$ não divide mult $\left(b_{n}(X)\right)$, o resultado segue.

Caso contrário, repetimos o processo e obtemos um automorfismo $\Phi_{2}$ tal que

$$
\Phi_{2}(f(X, Y))=c_{0}(X) Y^{n}+c_{1}(X) Y^{n-1}+\ldots+c_{n}(X)+Y^{n+1} h^{\prime \prime \prime}(X, Y),
$$

com $c_{0}(0) \neq 0$ e mult $\left(c_{n}(X)\right)>$ mult $\left(b_{n}(X)\right)$.

Este processo deve parar em algum estágio porque, caso contrário, obteríamos um automorfismo $\Phi$ tal que

$$
\Phi(f)=a_{0}^{\prime}(X) Y^{n}+a_{1}^{\prime}(X) Y^{n-1}+\ldots+a_{n-1}^{\prime}(X) Y+Y^{n+1} \widetilde{h}(X, Y)
$$

que é redutivel, o que seria uma contradição.

Proposição 5.2.5 Seja $(f)$ irredutivel com cone tangente $\left(Y^{n}\right)$. Seja $f^{(1)}=\sigma^{*}(f)$ e $f^{(i)}=\sigma^{*}\left(f^{(i-1)}\right)$. Se $n$ não divide $m:=I(f, Y)$, então

$$
\left[\frac{m}{n}\right]=\min \left\{i ; \operatorname{mult}\left(f^{(i)}\right) \neq \operatorname{mult}(f)\right\}
$$

onde $\left[\frac{m}{n}\right]$ é o maior inteiro.menor que $\frac{m}{n}$.

\section{Demonstração:}

Como $n$ não divide $m$ e $m>n$, do algoritmo da divisão temos

$$
m=n q+r, \quad \text { com } 0<r<n \text { e } q=\left[\frac{m}{n}\right] .
$$

Se $q=1$, então $m-n=r<n$. Da Proposição 5.2 .3 (iii), temos que mult $\left(f^{(1)}\right)<\operatorname{mult}(f)$ e, portanto, segue o resultado.

Se $q \geq 2$, então $m \geq 2 n+r>2 n$, logo $m-n>n$.

Suponhamos primeiramente $q=2$. Aplicando a Proposição 5.2.3 temos

- $\operatorname{mult}\left(f^{(1)}\right)=\operatorname{mult}(f)=n$

- $I\left(f^{(1)}, Y_{1}\right)=m-n$

- $I\left(f^{(2)}, Y_{2}\right)=I\left(f^{(1)}, Y_{1}\right)-\operatorname{mult}\left(f^{(1)}\right)=(m-n)-n=m-2 n=r<n$

- $\operatorname{mult}\left(f^{(2)}\right)=m-2 n<\operatorname{mult}\left(f^{(1)}\right)$ 
portanto, $q=2=\min \left\{i ; \operatorname{mult}\left(f^{(i)}\right) \neq\right.$ mult $\left.(f)\right\}$.

Se $q=3$ então $m-2 n \geq n$ e segue da Proposição 5.2.3 que

- $\operatorname{mult}\left(f^{(2)}\right)=\operatorname{mult}\left(f^{(1)}\right)=n$

- $I\left(f^{(3)}, Y_{3}\right)=I\left(f^{(2)}, Y_{2}\right)-\operatorname{mult}\left(f^{(2)}\right)=(m-2 n)-n=m-3 n=r<n$

- $\operatorname{mult}\left(f^{(3)}\right)=m-3 n<\operatorname{mult}\left(f^{(2)}\right)=\operatorname{mult}\left(f^{(1)}\right)=n$

Prosseguindo desta forma, temos

$$
I\left(f^{(q)}, Y_{q}\right)=I\left(f^{(q-1)}, Y_{q-1}\right)-\operatorname{mult}\left(f^{(q-1)}\right)=m-q n=r<n
$$

e

$$
\text { mult }\left(f^{(q)}\right)<\operatorname{mult}\left(f^{(q-1)}\right)=\ldots=\operatorname{mult}(f) .
$$

Vamos agora descrever o processo de desingularização de uma curva plana algebróide irredutivel que consiste em transformar uma curva singular em uma curva regular, através de sucessivos e convenientes blowing-ups.

Seja $f \in k[[X, Y]]$ irredutível de multiplicidade $n$, regular em $Y$ e tal que $n$ não divide $m=I(f, Y)$. Sejam $f^{(1)}=\sigma^{*}(f)$ e $f^{(i)}=\sigma^{*}\left(f^{(i-1)}\right)$, para $i=1, \ldots, q=\left[\frac{m}{n}\right]$. Vimos na proposição acima que mult $\left(f^{(i)}\right)=\operatorname{mult}(f)$, para $i=1, \ldots, q$, e $n^{\prime}=\operatorname{mult}\left(f^{(q)}\right)<n=\operatorname{mult}(f)$.

Se $n^{\prime}=1$, então a curva $f^{(q)}$ é regular e o processo para.

Se $n^{\prime}>1$, da Proposição 5.2.3(iii) temos que $f^{(q)}$ tem cone tangente $\left(X_{q}^{n^{\prime}}\right)$ e, portanto, é uma série de potências regular de ordem $n^{\prime}$ na indeterminada $X_{q}$. Sejam $m^{\prime}=I\left(f^{(q)}, X_{q}\right)$ e $q^{\prime}=\left[\frac{m^{\prime}}{n^{\prime}}\right]$. Segue do Lema 5.2.4 que, a memos de mudança de coordenadas, podemos supor que $m^{\prime}$ não é múltiplo de $n^{\prime}$.

Definimos $f^{(q+1)}=\tau^{*}\left(f^{(q)}\right)$ e $f^{(i)}=\tau^{*}\left(f^{(i-1)}\right), i=q+2, \ldots, q+q^{\prime}$. Observemos que mult $\left(f^{(i)}\right)=$ mult $\left(f^{(q)}\right), i=q+1, \ldots, q+q^{\prime}-1$.

Se $n^{\prime \prime}=\operatorname{mult}\left(f^{\left(q+q^{\prime}\right)}\right)=1$, então a curva $f^{\left(q+q^{\prime}\right)}$ é regular e o processo para.

Șe $n^{\prime \prime}>1$ da Proposição 5.2.3(iii) temos que $f^{\left(q+q^{\prime}\right)}$ é uma série de potências regular de ordem: $n^{\prime \prime}$ na indeterminada $Y_{q+q^{\prime}}$, e repetimos o processo usando $\sigma$. Como as multiplicidades são decrescentes, em algum ponto teremos mult $\left(f^{(N)}\right)=1$, ou seja, $\left(f^{(N)}\right)$ é uma curva regular.

A sequência $f=f^{(0)}, f^{(1)}, \ldots, f^{(N)}$ é chamada resolução canônica de $(f)$. Assim; obtemos o seguinte resultado:

Teorema 5.2.6 A resolução canônica de uma curva algebróide plana irredutivel é finita e a curva se transforma em uma curva não-singular (regular).

A resolução canônica de $(f)$ determina a sequência númerica

$$
\text { mult }(f) \geq \operatorname{mult}\left(f^{(1)}\right) \geq \ldots \geq \operatorname{mult}\left(f^{(i)}\right) \geq \ldots \geq \operatorname{mult}\left(f^{(N)}\right)=1
$$

que será chamada de sequência de multiplicidades de $(f)$.

Dizemos que dois ramos planos são equisolúveis se suas sequências de multiplicidades são iguais.

Exemplo 5.2.7 Vamos determinar a resolução canônica de $f=Y^{4}-X^{7}$. Temos que $f^{(1)}=\sigma^{*}(f)=$ $\frac{1}{X_{1}^{4}} f\left(X_{1}, X_{1} Y_{1}\right)=Y_{1}^{4}-X_{1}^{3}$ e mult $\left(f^{(1)}\right)=3$. Aplicando $\tau^{*}$ à $f^{(1)}$, obtemos $f^{(2)}=\frac{1}{Y_{2}^{3}} f^{(1)}\left(X_{2} Y_{2}, Y_{2}\right)=$ $Y_{2}-X_{2}^{3}$ que é não-singular. A sequência de multiplicidades neste caso é $(4,3,1)$. 


\subsection{Fórmula de Noether}

Nesta seção vamos relacionar o índice de interseção de duas curvas algebróides irredutiveis planas $f$ e $g$ com o índice de interseção de suas respectivas transformadas estritas, usando as sequências de multiplicidades de $f$ e $g$.

Seja $f \in k[[X, Y]]$ uma série irredutível de multiplicidade $n$ e regular em $Y$. Vimos na Seção 3.4 que $f$ tem uma parametrização de Puiseux $\left(T^{n}, \varphi(T)\right) \operatorname{com} \varphi(T) \in k[[T]]=k\left[\left[X^{\frac{1}{n}}\right]\right]$ e $m=\operatorname{mult}(\varphi(T)) \geq$ $n$. De acordo com o Lema 5.2.4, podemos assumir que $m$ não é múltiplo de $n$.

Consideremos

$$
\sigma^{*}(f)=f^{(1)}\left(X_{1}, Y_{1}\right)=\frac{1}{X_{1}^{n}} f\left(X_{1}, X_{1} Y_{1}\right)
$$

Como $f^{(1)}\left(X_{1}, Y_{1}\right)$ é regular em $Y_{1}$ de ordem $n$ e

$$
f^{(1)}\left(T^{n}, \frac{\varphi(T)}{T^{n}}\right)=\frac{1}{\left(T^{n}\right)^{n}} f\left(T^{n}, \varphi(T)\right)=0
$$

segue que

$$
\left(T^{n}, \psi(T)\right):=\left(T^{n}, \frac{\varphi(T)}{T^{n}}\right)
$$

é uma parametrização de $f^{(1)}=\sigma^{*}(f)$. É possivel mostrar que $\left(T^{n}, \psi(T)\right)$ é uma parametrização primitiva de $f^{(1)}$.

Da Proposição 4.3.6, para $g \in k\left[\left[X_{1}, Y_{1}\right]\right]$ sempre temos

$$
I\left(\sigma^{*}(f), g\right)=\operatorname{mult}\left(g\left(T^{n}, \psi(T)\right)\right)
$$

Vimos na Seção 4.1 que temos um homomorfismo injetor

$$
\begin{aligned}
H_{\varphi}: \mathcal{O}_{f} & \longrightarrow k[[T]] \\
\bar{g} & \longmapsto g\left(T^{n}, \varphi(T)\right)
\end{aligned}
$$

que nos permite identificar $\mathcal{O}_{f}$ com a subálgebra de $k[[T]]$, dada por $A_{\varphi}:=H_{\varphi}\left(\mathcal{O}_{f}\right)=k\left[\left[T^{n}, \varphi(T)\right]\right]$. Analogamente, temos um homomorfismo injetor

$$
\begin{aligned}
H_{\psi}: \mathcal{O}_{f^{(1)}} & \longrightarrow k[[T]] \\
\overline{\bar{g}} & \longmapsto g\left(T^{n}, \psi(T)\right)
\end{aligned}
$$

que nos permite identificar $\mathcal{O}_{f^{(1)}} \operatorname{com} A_{\psi}=k\left[\left[T^{n}, \psi(T)\right]\right]$. O resultado seguinte nos mostra que existe um homomorfismo injetor do anel local de uma curva plana algebróide irredutível no anel local de seu blowing-up.

Proposição 5.3.1 Seja $f \in k[[X, Y]]$ irredutivel com cone tangente $Y^{n}$. Então existe um homomorfismo injetor $\phi$ de modo que o seguinte diagrama é comutativo

$$
\begin{array}{rll}
\mathcal{O}_{f} & \stackrel{\phi}{\hookrightarrow} \mathcal{O}_{f^{(1)}} \\
H_{\varphi} \downarrow & & \downarrow H_{\psi} \\
A_{\varphi} & \stackrel{I d}{\hookrightarrow} A_{\psi}
\end{array}
$$




\section{Demonstração:}

Definimos $\phi: \mathcal{O}_{f} \rightarrow \mathcal{O}_{f^{(1)}}$ por $\phi(\overline{g(X, Y)})=\overline{\overline{g\left(X_{1}, X_{1} Y_{1}\right)}}$. Observemos que $\phi$ pode ser visto como um homomorfismo de $k[[X]]$-módulos

$$
\begin{aligned}
\phi: \quad k[[X]] \oplus \ldots \oplus k[[X]] y^{n-1} & \longrightarrow k\left[\left[X_{1}\right]\right] \oplus \ldots \oplus k\left[\left[X_{1}\right]\right] y_{1}^{n-1} \\
g(X, y) & \longmapsto g\left(X_{1}, X_{1} y_{1}\right)
\end{aligned}
$$

De fato, seja $\overline{g(X, Y)}=a_{0}(X)+a_{1}(X) y+\ldots+a_{n-1}(X) y^{n-1} \in \mathcal{O}_{f}$ então

$$
g(X, Y)=f(X, Y) q(X, Y)+a_{0}(X)+a_{1}(X) Y+\ldots+a_{n-1}(X) Y^{n-1} .
$$

Segue que

$$
\begin{aligned}
g\left(X_{1}, X_{1} Y_{1}\right) & =f\left(X_{1}, X_{1} Y_{1}\right) q\left(X_{1}, X_{1} Y_{1}\right)+a_{0}\left(X_{1}\right)+a_{1}\left(X_{1}\right) X_{1} Y_{1}+\ldots+a_{n-1}\left(X_{1}\right) X_{1}^{n-1} Y_{1}^{n-1}= \\
= & X_{1}^{n}\left(\frac{f\left(X_{1}, X_{1} Y_{1}\right) q\left(X_{1}, X_{1} Y_{1}\right)}{X_{1}^{n}}+\frac{a_{0}\left(X_{1}\right)}{X_{1}^{n}}+\frac{a_{1}\left(X_{1}\right) Y_{1}}{X_{1}^{n-1}}+\ldots+\frac{a_{n-1}\left(X_{1}\right) Y_{1}^{n-1}}{X_{1}}\right) .
\end{aligned}
$$

Observando que $f^{(1)}\left(X_{1}, Y_{1}\right)=\frac{1}{X_{1}^{n}} f\left(X_{1}, X_{1} Y_{1}\right)$, temos que

$$
\overline{\overline{g\left(X_{1}, X_{1} Y_{1}\right)}}=a_{0}\left(X_{1}\right)+a_{1}\left(X_{1}\right) X_{1} y_{1}+\ldots+a_{n-1}\left(X_{1}\right) X_{1}^{n-1} y_{1}^{n-1}
$$

Visto como um homomorfismo de $k[[X]]-$ módulos, $\phi$ é bem definida e injetora.

Seja $g(X, y) \in \mathcal{O}_{f}$, então

$$
H_{\psi}(\phi(g(X, y)))=H_{\psi}\left(g\left(X_{1}, X_{1} y_{1}\right)\right)=g\left(T^{n}, T^{n} \psi(T)\right)=g\left(T^{n}, \varphi(T)\right)=H_{\varphi}(g)
$$

Por outro lado,

$$
I d\left(H_{\varphi}(g)\right)=H_{\varphi}(g)=g\left(T^{n}, \varphi(T)\right)=g\left(T^{n}, T^{n} \psi(T)\right)=H_{\psi}(\phi(g)) .
$$

Logo, o diagrama é comutativo.

Proposição 5.3.2 Sejam $(f)$ e $(g)$ duas curvas algebróides planas com $f$ irredutivel. Então

$$
I(f, g)=\operatorname{mult}(f) \text { mult }(g)+I\left(f^{(1)}, g^{(1)}\right) .
$$

\section{Demonstração:}

Denotemos por $n$ e $n^{\prime}$ as multiplicidades de $f$ e $g$, respectivamente. Podemos supor, através de mudança de coordenadas, que $f$ é regular em $Y$. Seja $\left(T^{n}, \varphi(T)\right)$ uma parametrização de Puiseux de $(f)$. Então $\left(T^{n}, \frac{\varphi(T)}{T^{n}}\right)$ é uma parametrização primitiva de $f^{(1)}$. Como $g^{(1)}=\frac{1}{X_{1}^{n^{\prime}}} g\left(X_{1}, X_{1} Y_{1}\right)$, obtemos

$$
I\left(f^{(1)}, g^{(1)}\right)=\operatorname{mult}\left(\frac{1}{\left(T^{n}\right)^{n^{\prime}}} g\left(T^{n}, T^{n} \frac{\varphi(T)}{T^{n}}\right)\right)=-n n^{\prime}+\operatorname{mult}\left(g\left(T^{n}, \varphi(T)\right)=-n n^{\prime}+I(f, g) .\right.
$$

Portanto,

$$
I(f, g)=n n^{\prime}+I\left(f^{(1)}, g^{(1)}\right)=\text { mult }(f) \text { mult }(g)+I\left(f^{(1)}, g^{(1)}\right)
$$

Vamos agora demonstrar o principal resultado desta seção, uma fórmula devida a Max Noether, que relaciona o índice de interseção entre duas curvas algebróides planas irredutíveis e as multiplicidades de suas transformadas estritas. 
Teorema 5.3.3 (Fórmula de Noether) Sejam $f$ e $g$ duas curvas algebróides planas irredutíveis. Temos que

$$
I(f, g)=\sum_{i=0}^{N} \operatorname{mult}\left(f^{(i)}\right) \operatorname{mult}\left(g^{(i)}\right)
$$

onde $f^{(0)}=f$ e $g^{(0)}=g$.

\section{Demonstração:}

Vimos na proposição anterior que

$$
I(f, g)=\operatorname{mult}(f) \operatorname{mult}(g)+I\left(f^{(1)}, g^{(1)}\right)
$$

se escolhermos $\sigma$ ou $\tau$ de forma que as curvas $f^{(1)}$ e $g^{(1)}$ sejam irredutíveis e regulares em $Y$. Aplicamos a Proposição 5.3.2 até obtermos uma sequência finita, digamos de comprimento $N$, de blowing-ups do tipo $\sigma$ ou $\tau$, conforme o caso, de forma que $f^{(N)}$ e $g^{(N)}$ tem tangentes distintas. Então

$I(f, g)=\operatorname{mult}(f)$ mult $(g)+\operatorname{mult}\left(f^{(1)}\right)$ mult $\left(g^{(1)}\right)+\ldots+\operatorname{mult}\left(f^{(N-1)}\right)$ mult $\left(g^{(N-1)}\right)+I\left(f^{(N)}, g^{(N)}\right)$.

Usando agora o Teorema 4.3.7 e o fato de que $f^{(N)}$ e $g^{(N)}$ possuem tangentes distintas concluimos que

$$
I(f, g)=\sum_{i=0}^{N} \text { mult }\left(f^{(i)}\right) \text { mult }\left(g^{(i)}\right),
$$

onde $f^{(0)}=f$ e $g^{(0)}=g$ 


\section{Capítulo 6}

\section{Semigrupos de Ramos Planos}

Primeiramente vamos definir semigrupos do conjunto dos números naturais e obter algumas propriedades. A seguir introduziremos o semigrupo de valores de uma curva algebróide plana irredutível, que é um tipo especial de semigrupo dos Naturais e é um invariante da curva. O semigrupo de valores de uma curva algebróide plana irredutivel é também um invariante topológico no caso de curvas analíticas planas irredutíveis complexas. Por fim, relacionaremos este semigrupo aos inteiros característicos e aos pares de Puiseux, que são invariantes numéricos da curva.

\subsection{Semigrupos dos Naturais}

Seja $G$ um subconjunto de $\mathbb{N}, 0 \in G$ e $G \neq\{0\}$. Dizemos que $G$ é um semigrupo de $\mathbb{N}$ se $G$ é fechado em relação à adição.

O elemento $\min (G \backslash\{0\})$ será chamado de multiplicidade de $G$ e será denotado por mult $(G)$.

Se $x_{0}, \ldots, x_{r} \in \mathbb{N}$ então o conjunto

$$
\left\langle x_{0}, \ldots, x_{r}\right\rangle=\left\{\lambda_{0} x_{0}+\ldots+\lambda_{r} x_{r} ; \lambda_{0}, \ldots, \lambda_{r} \in \mathbb{N}\right\}
$$

é claramente um semigrupo de $\mathbb{N}$ chamado de semigrupo gerado por $x_{0}, \ldots, x_{r}$. Os elementos $x_{0}, \ldots, x_{r}$ são chamados de geradores de $G$.

Proposição 6.1.I Dado um semigrupo $G$ de $\mathbb{N}$ qualquer, existe um único subconjunto $\left\{v_{0}, \ldots, v_{g}\right\} \subset$ $G$ satisfazendo

1. $v_{0}<\ldots<v_{g}$ e $v_{i} \neq v_{j} \bmod v_{0}$, para $i \neq j$.

2. $G=\left\langle v_{0}, \ldots, v_{g}\right\rangle$.

3. $\left\{v_{0}, \ldots, v_{g}\right\}$ está contido em qualquer conjunto de geradores de $G$.

\section{Demonstração:}

Obteremos $v_{0}, \ldots, v_{g}$ como segue. Tomando $v_{0}=\operatorname{mult}(G)$, definimos $v_{1}=\min \left(G \backslash\left\langle v_{0}\right\rangle\right)$. Assim, $v_{0} \neq v_{1} \bmod v_{0}$.

Definimos

$$
v_{i}=\min \left(G \backslash\left\langle v_{0}, \ldots, v_{i-1}\right\rangle\right)
$$


para todo $i \geq 2$. Temos que $v_{i} \not \equiv v_{j} \bmod v_{0}, \forall j<i$, pois caso contrário, $v_{i} \in\left\langle v_{0}, \ldots, v_{i-1}\right\rangle$, o que é uma contradição.

Observemos que o número máximo de escolhas para os $v_{i}^{\prime} s$ é $v_{0}-1$, logo este processo para.

O conjunto $\left\{v_{0}, \ldots, v_{g}\right\}$ da proposição acima será chamado de sistema minimal de geradores de $G$ e o inteiro $g$ será chamados de gênero do semigrupo $G$. Observemos que $g \leq \operatorname{mult}(G)-1$.

Dado um semigrupo $G$ de $\mathbb{N}$, os elementos de $\mathbb{N} \backslash G$ são chamados de lacunas de $G$. Um semigrupo de $\mathbb{N}$ pode possuir finitas ou infinitas lacunas.

Exemplo 6.1.2 O semigrupo $\langle 2\rangle=\{0,2,4,6, \ldots\}$ tem infinitas lacunas e as lacunas do semigrupo $\langle 3,5\rangle=\{3,5,6,8,9,10,11, \ldots\}$ são $1,2,4$ e 7 .

Quando o número de lacunas de $G$ é finito, existe um único elemento $c \in G$, chamado de condutor de $G$, tal que

- $c-1 \notin G$.

- Se $z \in \mathbb{N}$ e $z \geq c$, então $z \in G$.

No exemplo acima o condutor do semigrupo $\langle 3,5\rangle$ é 8 . Se $n>1$, o semigrupo $\langle n\rangle$ não possui condutor.

Proposição 6.1.3 Seja $G$ um semigrupo de $\mathbb{N}$. As seguintes afirmações são equivalentes

(i) G tem condutor.

(ii) mdc $G=1$, ou seja, existem $\alpha_{1}, \ldots, \alpha_{m} \in G$ tais que mdc $\left(\alpha_{1}, \ldots, \alpha_{m}\right)=1$.

(iii) Existem dois inteiros consecutivos em $G$.

\section{Demonstração:}

Se $G$ tem condutor, digamos $c$, então $c, c+1 \in G$. Logo, $\operatorname{mdc} G=1$, pois $\operatorname{mdc}(c, c+1)=1$.

Seja $\left\{v_{0}, \ldots, v_{g}\right\}$ o sistema minimal de geradores de $G$. Suponhamos que mdc $G=1$, ou seja, existem $\alpha_{1}, \ldots, \alpha_{m} \in G$ tais que mdc $\left(\alpha_{1}, \ldots, \alpha_{m}\right)=1$. Logo, existem inteiros $\lambda_{1}, \ldots, \lambda_{m}$ tais que $\lambda_{1} \alpha_{1}+\ldots+\lambda_{m} \alpha_{m}=1$. Como $\alpha_{1}, \ldots, \alpha_{m} \in G=\left\langle v_{0}, \ldots, v_{g}\right\rangle$, existem inteiros $\beta_{0}, \ldots, \beta_{g}$ tais que $\beta_{0} v_{0}+\ldots+\beta_{g} v_{g}=1$. Transferindo para o segundo membro da equação os termos cujos $\beta_{i}$ 's são negativos, segue que existem dois inteiros consecutivos em $G$. Se não existir $\beta_{i}$ negativo, então $1 \in G$ e, portanto, $G=\mathbb{N}$.

Sejam agora $a$ e $a+1$ dois elementos em $G$. Então qualquer inteiro $n \geq(a-1)(a+1) \in G$. De fato,

$$
\begin{aligned}
a^{2}-1= & (a-1)(a+1) \in G \\
& a^{2}=a a \in G \\
a^{2}+1= & (a+1)+(a-1) a \in G
\end{aligned}
$$




$$
\begin{gathered}
a^{2}+m=m(a+1)+(a-m) a \in G \\
\vdots \\
a^{2}+(a-1)=(a-1)(a+1)+(a-a+1) a \in G .
\end{gathered}
$$

Como $a \in G$, segue que todo $n \geq(a-1)(a+1) \in G$, pois $a^{2}+a \in G, a^{2}+a+1 \in G, \ldots, a^{2}+2 a-1 \in G$ e assim sucessivamente. Assim, $G$ possui um condutor $c \leq(a-1)(a+1)$.

A proposição acima nos dá uma estimativa para o condutor $c$ de $G$, isto é, $c \leq(a-1)(a+1)$, quando $a$ e $a+1$ são elementos de $G$.

Seja $G=\left\langle x_{0}, \ldots, x_{r}\right\rangle$ um semigrupo de $\mathbb{N}$ com condutor $c$. Vamos definir duas sequências finitas de números naturais associadas ao conjunto de geradores de $G$. Sejam $e_{0}=x_{0}$ e $n_{0}=1$. Para $i=1, \ldots, r$ definimos

$$
e_{i}=\operatorname{mdc}\left(x_{0}, \ldots, x_{i}\right) \text { e } n_{i}=\frac{e_{i-1}}{e_{i}} .
$$

Como claramente $e_{i}$ divide $e_{i-1}, n_{i}$ é um inteiro, para todo $i=1, \ldots, r$. Além disso, segue da proposição acima que $e_{r}=\operatorname{mdc}\left(x_{0}, \ldots, x_{r}\right)=1$.

\subsection{Semigrupos de valores}

No que segue, $f$ será um elemento irredutível em $\mathcal{M} \subset k[[X, Y]]$. Portanto, o anel $\mathcal{O}_{f}=\frac{k[[X, Y]]}{\langle f\rangle}$ é um domínio. Seja $v$ a valorização associada a $f$ definida na Seção 4.1 por

$$
\begin{aligned}
v_{f}: \mathcal{O}_{f} \backslash\{0\} & \longrightarrow \mathbb{N} \\
\bar{g} & \longmapsto \operatorname{mult}\left(H_{p}(\bar{g})\right)=\operatorname{mult}\left(g\left(T^{n}, \varphi(T)\right)\right)
\end{aligned}
$$

$\operatorname{com} v_{f}(\overline{0})=\infty$.

Para simplificar a notação, vamos denotar a classe residual de $Y$ módulo $\langle f\rangle$ por $y$ e a classe de $X$ por $x$.

Definição 6.2.1 O semigrupo de valores associado a uma curva irredutivel $(f)$ é o conjunto

$$
S(f)=\{I(f, g) ; g \in k[[X, Y]] \backslash\langle f\rangle\} \subset \mathbb{N} .
$$

Se duas curvas algebróides irredutíveis planas $f$ e $g$ são equivalentes, então $S(f)=S(g)$. De fato, se existe um $k$-automorfismo $\phi$ e uma unidade $u \in k[[X, Y]]$ tais que $\phi(g)=u f$, então $I(g, h)=$ $I(\phi(g), \phi(h))=I(u f, \phi(h))=I\left(u^{-1} u f, u^{-1} \phi(h)\right)=I\left(f, h^{\prime}\right)$. Se $h^{\prime} \in\langle f\rangle$ então $I(g, h)=\infty$, donde $h \in\langle g\rangle$. Assim, $S(g) \subset S(f)$. Analogamente, $S(f) \subset S(g)$.

O semigrupo de valores de uma curva também pode ser definido como segue. Seja $f$ uma série de potências irredutível em $k[[X, Y]]$ regular em $Y$, com parametrização de Puiseux dada por $\left(T^{n}, \varphi(T)\right)$. Então $S(f)=\left\{v_{f}(\bar{g}) ; \bar{g} \in \mathcal{O}_{f} \backslash\{0\}\right\}=\left\{\operatorname{mult}\left(H_{\varphi}(\bar{g})\right) ; \bar{g} \in \mathcal{O}_{f} \backslash\{0\}\right\}=\left\{\right.$ mult $\left.(h) ; h \in A_{\varphi} \backslash\{0\}\right\}$.

Dois ramos $(f)$ e $(g)$ serão chamados equisingulares se $S(f)=S(g)$. Se $(f)$ e $(g)$ são equivalentes então são equisingulares.

O semigrupo de valores de um ramo plano tem condutor. De fato, como ramos planos equivalentes possuem o mesmo semigrupo de valores, podemos assumir que o ramo é representado por um polinômio de Weierstrass $f$. Do Teorema 4.1.4, temos que qualquer elemento de $k[[T]]$ de multiplicidade maior 
que mult $\left(D_{Y}(f)\left(T^{n}\right)\right)$ está em $A_{\varphi}$ e, portanto, sua multiplicidade está em $S(f)$. Uma outra maneira de mostrar este fato provém da observação anterior ao Teorema 4.1.4, que diz que o corpo de frações de $A_{\varphi}$ é $k((T))$. Assim, existem $h_{1}, h_{2} \in A_{\varphi}$ tais que $T=\frac{h_{1}}{h_{2}}$. Logo, mult $\left(h_{1}\right)=\operatorname{mult}\left(h_{2}\right)+1 \mathrm{e}$, portanto, segue da Proposição 6.1.3 que $S(f)$ tem condutor.

Exemplos 6.2.2 1. Seja $f=Y^{2}-X^{3}$. Então $S(f)=\{0,2,3,4,5, \ldots\}$. De fato, temos que $X \notin\langle f\rangle$, portanto $v_{f}(x)=I(f, X)=I\left(Y^{2}-X^{3}, X\right)=I\left(Y^{2}, X\right)=2 I(Y, X)=2 \in S(f)$. Também temos que $Y \notin\langle f\rangle$, logo $v_{f}(y)=I(f, Y)=I\left(Y^{2}-X^{3}, Y\right)=I\left(X^{3}, Y\right)=3 I(X, Y)=$ $3 \in S(f)$. Como 2 e 3 estão em $S(f)$, segue da Proposição 6.1.3 que o condutor é menor ou igual a $(2-1)(2+1)=3$. Mas, $2 \in S(f)$ e, portanto, o condutor de $S(f)$ é 2 .

2. Seja $f=Y^{2}-X^{5}$. Então $S(f)=\{0,2,4,5,6, \ldots\}$. De fato, temos que $X \notin\langle f\rangle$, portanto $v_{f}(x)=I(f, X)=I\left(Y^{2}-X^{5}, X\right)=I\left(Y^{2}, X\right)=2 \in S(f)$. Também temos que $Y \notin\langle f\rangle$, logo $v_{f}(y)=I(f, Y)=I\left(Y^{2}-X^{5}, Y\right)=I\left(X^{5}, Y\right)=5 \in S(f)$. Qualquer número natural $n \geq 4$ pode ser escrito como $n=2 r+5 s$, com $r, s \in \mathbb{N}$, pois se $n$ é ímpar então $n=2 \alpha+5$, com $\alpha \geq 0$. Portanto, $v_{f}\left(x^{r} y^{s}\right)=I\left(f, X^{r} Y^{s}\right)=I\left(f, X^{r}\right)+I\left(f, Y^{s}\right)=r I(f, X)+s I(f, Y)=2 r+5 s \in S(f)$, pois $X^{r} Y^{s} \notin\langle f\rangle$. Assim, o condutor de $S(f)$ é 4 .

3. Seja $f=Y^{4}-X^{7}$. Temos que $\left(T^{4}, T^{7}\right)$ é uma parametrização de Puiseux de $(f)$, pois mult $(f)=$ 4 e $f\left(T^{4}, T^{7}\right)=0$. Dado $g=\sum_{i, j} a_{i, j} X^{i} Y^{j} \in k[[X, Y]]$, temos que

$$
v_{f}(\bar{g})=\operatorname{mult}\left(\sum_{i, j} a_{i, j} T^{4 i} T^{7 j}\right)=\operatorname{mult}\left(\sum_{i, j} a_{i, j} T^{4 i+7 j}\right)=4 r+7 s
$$

para algum par de números naturais $r$ e $s$. Isto mostra que $S(f)$ é o semigrupo gerado por 4 e 7. Então $S(f)=\{0,4,7,8,11,12,14,15,16,18,19,20,21,22,23, \ldots\}$. O condutor de $S(f)$ é 18. Observemos que a estimativa para o condutor $c$ que a Proposição 6.1.3 nos dá é de que $c \leq(7-1)(7+1)=48$, que é muito distante do condutor.

Observemos que, na maioria dos casos, não é fácil determinar $S(f)$.

O menor elemento de $S(f) \backslash\{0\}$ é o inteiro mult $(f)$, pois do Teorema 4.3.7, temos que

$$
v_{f}(\bar{g})=I(f, g) \geq \operatorname{mult}(f) \operatorname{mult}(g) \geq \operatorname{mult}(f)
$$

para todo $g \in \mathcal{M}$, e se a reta $L$ não pertence ao cone tangente de $(f)$ então $v_{f}(\bar{L})=I(f, L)=$ mult $(f)$ mult $(L)=\operatorname{mult}(f)$.

Para estudar o semigrupo de valores de um ramo plano $(f)$, vamos assumir que car $k=0$ e que suas coordenadas foram escolhidas de modo que $f$ seja regular em $Y$ e que o cone tangente de $(f)$ seja $Y^{n}$. Assim, $f$ é da forma $f=a_{0}(X) Y^{n}+a_{1}(X) Y^{n-1}+\ldots+a_{n}(X)+Y^{n+1} h(X, Y)$, com $a_{0}(0) \neq 0 \mathrm{e}$ mult $\left(a_{i}\right)>i$. Em particular, $a_{n}(X)=c X^{m}+\ldots$ onde $m>n$ e podemos supor que $m$ não é múltiplo de $n$, pelo Lema 5.2.4. Seja

$$
\left\{\begin{array}{l}
X=T^{n} \\
Y=\varphi(T)=\sum_{i \geq m} b_{i} T^{i}, b_{m} \neq 0, m>n
\end{array}\right.
$$


com $n$ e $m$ como acima, uma parametrização de Puiseux de $(f)$. Observemos que nẹsta situação $m=\min (S(f) \backslash\langle n\rangle)$. De fato, $m \in S(f)$ pois $v_{f}(y)=I(f, Y)=$ mult $\left(a_{n}(X)\right)=m$. Por outro lado, se $g=\sum a_{\alpha, \beta} X^{\alpha} Y^{\beta} \in \mathcal{M}$, então $I(f, g)=\operatorname{mult}\left(\sum a_{\alpha, \beta} T^{n \alpha}(\varphi(T))^{\beta}\right)$. Disto segue que $\min (S(f) \backslash\langle n\rangle) \geq$ $m$. Mas $I(f, Y)=m$, logo $\min (S(f) \backslash\langle n\rangle)=m$. Assim, se $v_{0}, v_{1}, \ldots, v_{g}$ é um sistema minimal de geradores para $S(f)$ então $v_{0}=n$ e $v_{1}=m$.

Sejam $\beta_{0}, \beta_{1}, \ldots, \beta_{\gamma}$ os expoentes característicos da parametrização de Puiseux de $(f)$. Sabemos que $\beta_{0}=n$ e $\beta_{1}=m$. Vamos denotar por $\eta_{j}$ e $\varepsilon_{j}$ os inteiros correspondentes aos expoentes característicos como definido na Seção 3.4. Também denotamos por $U_{n}$ o grupo das $n$-ésimas raízes da unidade em $k$. Seja $G_{j}=U_{\varepsilon_{j}}=\left\{\zeta \in k ; \zeta^{\varepsilon_{j}}=1\right\}, j=0, \ldots, \gamma$. Portanto, $U_{n}=G_{0} \supset G_{1} \supset \ldots \supset G_{\gamma}=$ $\{1\}$.

Lema 6.2.3 Se $\zeta \in G_{j} \backslash G_{j+1}$ então $\zeta^{\beta_{j+1}} \neq 1$.

\section{Demonstração:}

Suponhamos $\zeta^{\beta_{j+1}}=1$. Então $\zeta^{\varepsilon_{j}}=\zeta^{\beta_{j+1}}=1$, pois $\zeta \in G_{j}$. Como $\varepsilon_{j+1}=\operatorname{mdc}\left(\varepsilon_{j}, \beta_{j+1}\right)$, existem $x, y \in \mathbb{Z}$ tais que $\varepsilon_{j+1}=\varepsilon_{j} x+\beta_{j+1} y$. Logo, $\zeta^{\varepsilon_{j+1}}=\zeta^{\varepsilon_{j} x+\beta_{j+1} y}=\zeta^{\varepsilon_{j} x} \zeta^{\beta_{j+1} y}=1$, o que é uma contradição pois $\zeta \notin G_{j+1}$.

Lema 6.2.4 Seja $\zeta \in G_{k-1} \backslash G_{k}$. Se $l \geq k$, então $\zeta G_{l} \subset G_{k-1} \backslash G_{k}$.

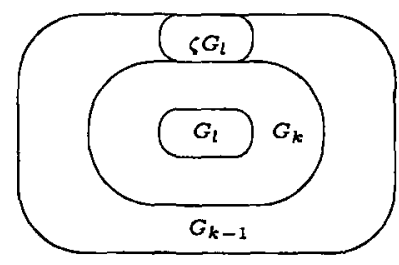

\section{Figura 1}

\section{Demonstraçāo:}

Temos que $\zeta^{\varepsilon_{k-1}}=1$ e $\zeta^{\varepsilon_{k}} \neq 1$. Seja $\rho$ um elemento de $G_{l}, l \geq k$. Como $l>k-1, \varepsilon_{l}$ divide $\varepsilon_{k-1}$ e $\varepsilon_{k}$. Assim,

$$
(\zeta \rho)^{\varepsilon_{k-1}}=\zeta^{\varepsilon_{k-1}} \rho^{\varepsilon_{l} m}=1 \text { e }(\zeta \rho)^{\varepsilon_{k}}=\zeta^{\varepsilon_{k}} \rho^{\varepsilon_{l} n}=\zeta^{\varepsilon_{k}} \neq 1
$$

Portanto, $\zeta \rho \in G_{k-1} \backslash G_{k}$.

Para todo $j=2, \ldots, \gamma$, definimos

$$
P_{j}(T)=\sum_{i=\beta_{1}}^{\beta_{j}-1} b_{i} T^{i}
$$

e $\beta_{\gamma+1}=\infty$. Então

$$
P_{\gamma+1}=\sum_{i=\beta_{1}}^{\infty} b_{i} T^{i}=Y .
$$


Consideremos agora, para todo $j=2, \ldots, \gamma, P_{j}\left(X^{\frac{1}{n}}\right) \in k\left(\left(X^{\frac{1}{n}}\right)\right)$. Queremos estudar as seguintes extensões:

$$
\left.\right|_{k((X))\left(P_{j}\left(X^{\frac{1}{n}}\right)\right)} ^{k(X))}
$$

Lema 6.2.5 Sejam $\zeta, \rho \in G_{0}=U_{n}$ e $j \in\{2, \ldots, \gamma\}$. Temos que $P_{j}(\zeta T)=P_{j}(\rho T)$ se, e somente se, $\zeta \rho^{-1} \in G_{j-1}$.

\section{Demonstração:}

Se $P_{j}(\zeta T)=P_{j}(\rho T)$ então

$$
\sum_{i=\beta_{1}}^{\beta_{j}-1} b_{i} \zeta^{i} T^{i}=\sum_{i=\beta_{1}}^{\beta_{j}-1} b_{i} \rho^{i} T^{i}
$$

Portanto, $\zeta^{i}=\rho^{i}$, para todo $i=n, \beta_{1}, \ldots, \beta_{j-1}$. Então $\zeta^{i} \rho^{-i}=1$ ou $\left(\zeta \rho^{-1}\right)^{i}=1$, para $i=$ $\operatorname{mdc}\left(n, \beta_{1}, \ldots, \beta_{j-1}\right)=\varepsilon_{j-1}$. Logo, $\zeta \rho^{-1} \in G_{j-1}$.

Reciprocamente, suponhamos que $\left(\zeta \rho^{-1}\right)^{\Sigma_{j-1}}=1$. Sabemos, da definição de $\beta_{j}$, que $\varepsilon_{j-1}$ divide todos os expoentes $i$ tais que $\beta_{j-1} \leq i<\beta_{j}$ e $b_{i} \neq 0$. Como $\varepsilon_{j-1}$ divide $\varepsilon_{l}$, para todo $l \leq j-1$, segue que $\varepsilon_{j-1}$ divide todos os expoentes $i$ tais que $\beta_{1} \leq i<\beta_{j}$ e $b_{i} \neq 0$. Então, $\zeta^{i} \rho^{-i}=\left(\zeta \rho^{-1}\right)^{i}=\left(\zeta \rho^{-1}\right)^{\varepsilon_{j-1} n_{i}}=1$, para todo $i=\beta_{1}, \beta_{1}+1, \ldots, \beta_{j}-1$, o que implica que $\zeta^{i}=\rho^{i}$ para todo $i=\beta_{1}, \beta_{1}+1, \ldots, \beta_{j}-1$, e $P_{j}(\zeta T)=P_{j}(\rho T)$.

Corolário 6:2.6 O grupo de Galois da extensão $k\left(\left(X^{\frac{1}{n}}\right)\right) / k((X))\left(P_{j}\left(X^{\frac{1}{n}}\right)\right)$ é $G_{j-1}$.

Como o grupo de Galois da extensão $k\left(\left(X^{\frac{1}{n}}\right)\right) / k((X))$ é $G_{0}=U_{n}$, que é um grupo abeliano, segue que o grupo de Galois de $k((X))\left(P_{j}\left(X^{\frac{1}{n}}\right)\right) / k((X))$ é $G_{0} / G_{j-1}$ (ver [3], Teorema 1.10, pag.265).

Denotando por [ $\zeta]$ a classe residual de $\zeta$ em $G_{0}=U_{n}$ módulo $G_{j-1}$, definimos $P_{j}([\zeta] T)=P_{j}(\zeta T)$, o que faz sentido pelo Lema 6.2.5. Assim, os conjugados de $P_{j}\left(X^{\frac{1}{n}}\right)$ em $k((X))^{*}$ sobre $k((X))$ são os $P_{j}\left([\zeta] X^{\frac{1}{n}}\right)$, onde $[\zeta] \in G_{0} / G_{j-1}$. Portanto,

$$
f_{j}(X, Y):=\prod_{[\zeta] \in G_{0}^{\dot{0}} / G_{j-1}}\left(Y-P_{j}\left([\zeta] X^{\frac{1}{n}}\right)\right) \in k[[X]][Y] .
$$

O polinômio $f_{j}(X, Y)$.é de grau $n / \varepsilon_{j-1}=\eta_{1} \eta_{2} \ldots \eta_{j-1}$ em $Y$, e é o polinômio minimal de $P_{j}\left(X^{\frac{1}{n}}\right)$ sobre $k((X))$. Ainda, $f_{j}=f_{j}(x, y) \in \mathcal{O}_{f}$.

Definimos

$$
v_{j}= \begin{cases}\beta_{0}=n & \text { se } j=0 \\ \beta_{1} & \text { se } j=1 \\ v\left(f_{j}\right)=I\left(f, f_{j}\right) & \text { se } j=2, \ldots, \gamma\end{cases}
$$

Portanto, os $v_{j}^{\prime}$ s são elementos de $S(f)$. O seguinte resultado vai relacionar estes inteiros aos expoentes característicos $\beta_{0}, \ldots, \beta_{\gamma}$ de $(f)$. Para enunciarmos o teorema vamos precisar da seguinte notação: definimos, para $k=0, \ldots, n$,

$$
M_{k}^{\prime}=k[[X]]+k[[X]] y+k[[X]] y^{2}+\ldots+k[[X]] y^{k} \subset \mathcal{O}_{f},
$$

onde $y$ é a classe residual de $Y$ em $\mathcal{O}_{f}$. Observemos que $M_{n-1}=\mathcal{O}_{f}$. 
Teorema 6.2.7 (i) Para $j=2, \ldots, \gamma$, temos que

$$
v_{j}=\sum_{k=1}^{j-1} \frac{\varepsilon_{k-1}-\varepsilon_{k}}{\varepsilon_{j-1}} \beta_{k}+\beta_{j}
$$

(ii) Se $h \in M_{k}$, com $k<\eta_{1} \eta_{2} \ldots \eta_{j}$, então $v(h) \in \sum_{i=0}^{j} v_{i} \mathbb{N}$. Em particular, $S(f)=\left\langle v_{0}, \ldots, v_{\gamma}\right\rangle$.

(iii) $O \operatorname{mdc}\left(\varepsilon_{j-1}, v_{j}\right)=\varepsilon_{j}$, para todo $j=1, \ldots, \gamma$. Além disso, $v_{j}$ é o menor elemento não nulo de $S(f)$ que não é divisivel por $\varepsilon_{j-1}$.

(iv) O gênero de $S(f)$ é $\gamma \dot{e}$ os inteiros $v_{0}, \ldots, v_{\gamma}$ constituem um sistema minimal de geradores.

\section{Demonstração:}

(i) Afirmamos que se $j>k$ então cada conjunto $G_{k-1} \backslash G_{k}$ é uma união disjunta de $\frac{\varepsilon_{k-1}-\varepsilon_{k}}{\varepsilon_{j-1}}$ classes módulo $G_{j-1}$. De fato, $G_{k-1} \backslash G_{k}$ tem $\varepsilon_{k-1}-\varepsilon_{k}$ elementos e os elementos de $\frac{G_{k-1} \backslash G_{k}}{G_{j-1}}$ são da forma $\zeta G_{j-1}$, com $\zeta \in G_{k-1} \backslash G_{k}$. Como $j-1 \geq k$, segue do Lema 6.2.4 que $\zeta G_{j-1} \subset G_{k-1} \backslash G_{k}$. Portanto, se $j>k, G_{k-1} \backslash G_{k}$ tem $\frac{\varepsilon_{k-1}-\varepsilon_{k}}{\varepsilon_{j-1}}$ classes módulo $G_{j-1}$. Além disso, temos que

$$
v\left(y-P_{j}([\zeta] T)\right)=\operatorname{mult}\left(Y-P_{j}(\zeta T)\right)=\operatorname{mult}\left(\sum_{i=\beta_{1}}^{\infty} b_{i} T^{i}-\sum_{i=\beta_{1}}^{\beta_{j}-1} b_{i} \zeta^{i} T^{i}\right) .
$$

Assim, $v\left(y-P_{j}([\zeta] T)\right)=\beta_{k}$, para todo $\zeta \in G_{k-1} \backslash G_{k}$, pois segue do Lema 6.2 .3 que $\zeta^{\beta_{k}} \neq 1$. Então

$$
v_{j}=v\left(f_{j}\right)=v\left(\prod_{[\zeta] \in G_{0} / G_{j-1}}\left(y-P_{j}([\zeta] T)\right)\right)=\sum_{k=1}^{j-1} \frac{\varepsilon_{k-1}-\varepsilon_{k}}{\varepsilon_{j-1}} \beta_{k}+\beta_{j} .
$$

(ii) A demonstração é por indução sobre $j$.

Se $j=1$, seja $h=a_{0}(X)+y a_{1}(X)+\ldots+y^{k} a_{k}(X), \operatorname{com} k<\eta_{1}=\frac{n}{\varepsilon_{1}}=\frac{n}{\operatorname{mdc}\left(n, \beta_{1}\right)}$. Para todo $i=0, \ldots, \eta_{1}-1$, temos que $v\left(y^{i} a_{i}(x)\right)=v\left(y^{i}\right)+v\left(a_{i}(x)\right)=i \beta_{1}+v\left(a_{i}(x)\right)$. Logo, $v\left(y^{i} a_{i}(x)\right) \equiv$ $i \beta_{1} \bmod n$.

Se $i$ e $l$ são inteiros distintos não-negativos menores que $\eta_{1}$ então $(i-l) \beta_{1} \equiv 0: \bmod n$, pois caso contrário, $(i-l) \frac{\beta_{1}}{\varepsilon_{1}}=\lambda \frac{n}{\varepsilon_{1}}=\lambda \eta_{1}$, para algum $\lambda$. Como $\frac{\beta_{1}}{\varepsilon_{1}}$ e $\eta_{1}=\frac{n}{\varepsilon_{1}}$ são relativamente primos, teríamos $\eta_{1}$ dividindo $i-l$, o que é uma contradição pois $0<|i-l|<\eta_{1}$.

Portanto, $v\left(y^{i} a_{i}(x)\right) \neq v\left(y^{l} a_{l}(x)\right)$, se $i, l<\eta_{1}$ com $i \neq l$. Segue que $v(h)=v\left(y^{i} a_{i}(x)\right)$ para algum $i=0, \ldots, k$. Assim, $v(h) \in n \mathbb{N}+\beta_{1} \mathbb{N}=v_{0} \mathbb{N}+v_{1} \mathbb{N}$.

Suponhamos que o resultado seja válido para $j-1$. Seja $h \in M_{k}$ e $k<\eta_{1} \eta_{2} \ldots \eta_{j}$. Suponhamos que $h=\overline{h(X, Y)}$, onde $h(X, Y)$ é um polinômio em $k[[X]][Y]$ de grau menor ou igual a $k$. Agrupando $h(X, Y)$ com respeito às potências de $f_{j}(X, Y)$, podemos escrever

$$
h(X, Y)=A_{0}(X, Y)+A_{1}(X, Y) f_{j}(X, Y)+\ldots+A_{s}(X, Y) f_{j}(X, Y)^{s},
$$


onde $A_{i}(X, Y) \in k[[X]][Y], \partial\left(A_{i}\right)<\partial\left(f_{j}(X, Y)\right)=\eta_{1} \eta_{2} \ldots \eta_{j-1}$ e $s<\eta_{j}, i=1, \ldots, s$. Portanto,

$$
h=A_{0}(x, y)+A_{1}(x, y) f_{j}+\ldots+A_{s}(x, y)\left(f_{j}\right)^{s},
$$

onde $A_{i} \in M_{k^{\prime}}$, com $k^{\prime}<\eta_{1} \eta_{2} \ldots \eta_{j-1}, i=0, \ldots, s$. Da hipótese de indução temos que, para $i=0, \ldots, s$,

$$
v\left(A_{i}(x, y)\right) \in \sum_{\nu=0}^{j-1} v_{\nu} \mathbb{N}
$$

Como $\varepsilon_{j-1}=\operatorname{mdc}\left(\beta_{0}, \ldots, \beta_{j-1}\right)$, segue de (6.2) e de $(i)$ que

$$
v\left(A_{i}(x, y)\right) \equiv 0 \bmod \varepsilon_{j-1}
$$

e

$$
v\left(A_{i}(x, y) f_{j}^{i}\right)=v\left(A_{i}(x, y)\right)+i v_{j} \equiv i v_{j} \bmod \varepsilon_{j-1}=i \beta_{j} \bmod \varepsilon_{j-1}
$$

Como $\frac{\beta_{j}}{\varepsilon_{j}}$ e $\eta_{j}=\frac{\varepsilon_{j-1}}{\varepsilon_{j}}$ são relativamente primos, segue analogamente ao caso $j=1$ que, se $i, l<\eta_{j}$ e $i \neq l$, então $(i-l) \beta_{j} \neq 0 \bmod \dot{\varepsilon}_{j-1}$, Logo, os $v\left(A_{i}(x, y) f_{j}^{i}\right)$ são distintos, $i=0, \ldots, s$. Portanto, existe $i \in\{0, \ldots, s\}$ tal que $v(h)=v\left(A_{i}(x, y) f_{j}^{i}\right)=v\left(A_{i}(x, y)\right)+i v_{j} \in \sum_{\nu=0}^{j} v_{\nu} \mathbb{N}$, por (6.2).

(iii) De (6.1) temos que

$$
\operatorname{mdc}\left(\varepsilon_{j-1}, v_{j}\right)=\operatorname{mdc}\left(\varepsilon_{j-1}, \sum_{k=1}^{j-1} \frac{\varepsilon_{k-1}-\varepsilon_{k}}{\varepsilon_{j-1}} \beta_{k}+\beta_{j}\right)=\rho .
$$

Como $\varepsilon_{j}=\operatorname{mdc}\left(\varepsilon_{j-1}, \beta_{j}\right)=\operatorname{mdc}\left(\beta_{0}, \ldots, \beta_{j}\right)$, então $\varepsilon_{j}|\rho . \operatorname{Mas} \rho| \varepsilon_{j-1}$ e $\varepsilon_{j-1}=\operatorname{mdc}\left(\beta_{0}, \ldots, \beta_{j-1}\right)$, $\operatorname{logo} \rho \mid \varepsilon_{j-1}, \beta_{j}$, o que implica que $\rho \mid \operatorname{mdc}\left(\varepsilon_{j-1}, \beta_{j}\right)=\varepsilon_{j}$. Temos que $v_{0}<v_{1}<\ldots<v_{\gamma}$ e que $\varepsilon_{j-1}$ divide $v_{0}, v_{1}, \ldots, v_{j-1}$. Suponhamos que $\varepsilon_{j-1}$ divide $v_{j}$. Mas mdc $\left(\varepsilon_{j-1}, v_{j}\right)=\varepsilon_{j}$. Assim $\varepsilon_{j-1}<\varepsilon_{j}$, o que é um absurdo.

(iv) Temos que $v_{0}=n=\min (S(f) \backslash\{0\}), v_{1}=\beta_{1}=\min (S(f) \backslash n \mathbb{N})$ e para todo $j=2, \ldots, \gamma$,

$$
v_{j}=\min \left(S(f) \backslash \sum_{\nu=0}^{j-1} v_{\nu} \mathbb{N}\right) .
$$

Segue de (ii) e (iii) que $v_{0}, v_{1}, \ldots, v_{\gamma}$ é um sistema minimal de geradores de $S(f)$. Em particular, $\gamma$ é o gênero $g$ de $S(f)$.

Do teorema acima, $S(f)=\left\langle v_{0}, \ldots, v_{g}\right\rangle$. Na Seção 6.1 definimos

$$
e_{i}=\operatorname{mdc}\left(v_{0}, v_{1}, \ldots, v_{i}\right) \text { e } n_{i}=\frac{e_{i-1}}{e_{i}} \text {. }
$$

Mas $\varepsilon_{i}$ divide $v_{0}, v_{1}, \ldots, v_{i}$, donde $\varepsilon_{i}$ divide $e_{i}$. Reciprocamente, $\varepsilon_{i}=\operatorname{mdc}\left(\beta_{0}, \beta_{1}, \ldots, \beta_{i}\right)$ e $e_{i}$ divide $\beta_{0}, \ldots, \beta_{i}$. Portanto; $e_{i}$ divide $\varepsilon_{i}$ e logo $e_{i}=\varepsilon_{i}$. Assim,

$$
n_{i}=\frac{e_{i-1}}{e_{i}}=\frac{\varepsilon_{i-1}}{\varepsilon_{i}}=\eta_{i}
$$


Em particular, como $\eta_{i}>1$, então $n_{i}>1$, para todo $i=0, \ldots, g$. Sabendo que $\frac{\varepsilon_{i-1}}{\varepsilon_{j}}=\eta_{i} \eta_{i+1} \ldots \eta_{j}$, temos

$$
\begin{gathered}
v_{j}=\frac{\varepsilon_{0}-\varepsilon_{1}}{\varepsilon_{j-1}} \beta_{1}+\frac{\varepsilon_{1}-\varepsilon_{2}}{\varepsilon_{j-1}} \beta_{2}+\ldots+\frac{\varepsilon_{j-2}-\varepsilon_{j-1}}{\varepsilon_{j-1}} \beta_{j-1}+\beta_{j}= \\
\left(\eta_{1} \eta_{2} \ldots \eta_{j-1}-\eta_{2} \eta_{3} \ldots \eta_{j-1}\right) \beta_{1}+\left(\eta_{2} \eta_{3} \ldots \eta_{j-1}-\eta_{3} \eta_{4} \ldots \eta_{j-1}\right) \beta_{2}+\ldots+\left(\eta_{j-1}-1\right) \beta_{j-1}+\beta_{j}= \\
\left(\eta_{1}-1\right) \eta_{2} \ldots \eta_{j-1} \beta_{1}+\left(\eta_{2}-1\right) \eta_{3} \ldots \eta_{j-1} \beta_{2}+\ldots+\left(\eta_{j-1}-1\right) \beta_{j-1}+\beta_{j}
\end{gathered}
$$

e

$$
\begin{gathered}
v_{j+1}=\left(\eta_{1}-1\right) \eta_{2} \ldots \eta_{j} \beta_{1}+\left(\eta_{2}-1\right) \eta_{3} \ldots \eta_{j} \beta_{2}+\ldots+\left(\eta_{j-1}-1\right) \eta_{j} \beta_{j-1}+\left(\eta_{j}-1\right) \beta_{j}+\beta_{j+1} \\
v_{j+1}=\eta_{j} v_{j}-\beta_{j}+\beta_{j+1}, j=1, \ldots, g-1
\end{gathered}
$$

Como a sequência dos $\beta_{i}^{\prime}$ s é crescente, segue de (6.4) que

$$
v_{j+1}>\eta_{j} v_{j}, j=1, \ldots, g-1 .
$$

Definição 6.2.8 Um semigrupo $S$ com um sistema minimal de geradores $v_{0}, v_{1}, \ldots, v_{g}$ satisfazendo a condição (6.5) será chamado de semigrupo fortemente crescente.

Logo, o semigrupo de valores de um ramo plano é um semigrupo fortemente crescente.

Teorema 6.2.9 Seja $f$ uma série de potências irredutivel em $k[[X, Y]]$ com uma dada parametrização de Puiseux. Então $S(f)$ e os inteiros característicos de $(f)$ se auto determinam. .

\section{Demonstração:}

Dados os inteiros característicos $\beta_{0}, \beta_{1}, \ldots, \beta_{g}$ de $(f)$ podemos determinar, através de (6.4), os inteiros $v_{0}, v_{1}, \ldots, v_{g}$ e, assim, $S(f)=v_{0}, v_{1}, \ldots, v_{g}$.

Reciprocamente, dado $S(f)$ então seu sistema minimal de geradores $v_{0}, v_{1}, \ldots, v_{g}$ é bem determinado e os inteiros $n_{i}$ são obtidos indutivamente: $e_{0}=n, e_{i}=\operatorname{mdc}\left(e_{i-1}, v_{i}\right)$ e $\eta_{i}=\frac{e_{i-1}}{e_{i}}$. Assim, os $\beta_{i}^{\prime} \mathrm{s}$ podem ser recursivamente determinados pela fórmula (6.4).

Teorema 6.2.10 Sejam $S$ um semigrupo com condutor e $v_{0}, v_{1}, \ldots, v_{g}$ um sistema minimal de geradores tal que $n_{i}>1$ e $v_{i}>n_{i-1} v_{i-1}$, para todo $i=1, \ldots, g$. Então existe uma curva algebróide plana irredutivel $(f)$ tal que $S(f)=S$.

\section{Demonstração:}

Definimos $n=\beta_{0}=v_{0}$ e $\beta_{1}=v_{1}$. Da equação (6.4) obtemos uma sequência de inteiros $\beta_{0}, \beta_{1}, \ldots, \beta_{g}$ que é crescente, pois $v_{i}>n_{i-1} v_{i-1}$. Como $n_{i}>1$ então mdc $\left(\beta_{0}, \beta_{1}, \ldots, \beta_{i-1}\right) \neq$ $\operatorname{mdc}\left(\beta_{0}, \beta_{1}, \ldots, \beta_{i}\right)$. Agora, sejam $\varphi(T)=T^{\beta_{1}}+T^{\beta_{2}}+\ldots+T^{\beta_{g}} \mathrm{e}$

$$
f(X, Y)=\prod_{i=1}^{n}\left(Y-\varphi\left(\zeta^{i} X^{\frac{1}{n}}\right)\right)
$$

onde $\zeta$ é uma raiz $n$-ésima primitiva da unidade. Então $f(X, Y) \in k[[X]][Y]$ determina uma curva algebróide plana irredutível $(f)$ com expoentes cạracterísticos $\beta_{0}, \beta_{1}, \ldots, \beta_{g}$ e com semigrupo de valores $S$. 


\subsection{Semigrupos e equações cartesianas}

Já vimos como determinar o semigrupo $S(f)$ através de uma parametrização de Puiseux de $(f)$. Agora vamos mostrar como determinar, através da equaçāo de $f$, os inteiros característicos da curva e, através da equação (6.4), os geradores de $S(f)$.

Seja $(f)$ uma curva plana algebróide irredutivel regular em $Y$ e parametrizada por

$$
\left\{\begin{array}{l}
X=T^{n} \\
Y=\varphi(T)=\sum_{i \geq m} b_{i} T^{i}, b_{m} \neq 0, m>n
\end{array}\right.
$$

Lema 6.3.1 Se $\zeta \in G_{k} \backslash G_{k+1}$ então mult $(\varphi(T)-\varphi(\zeta T))=\beta_{k+1}$.

\section{Demonstração:}

Podemos escrever $\varphi(T)=\sum_{i \geq m}^{\beta_{k+1}-1} b_{i} T^{i}+\sum_{i \geq \beta_{k+1}} b_{i} T^{i}$. Se $\zeta \in G_{k} \backslash G_{k+1}$, temos que

$$
\sum_{i \geq m}^{\beta_{k+1}-1} b_{i}(\zeta T)^{i}=\sum_{i \geq m}^{\beta_{k+1}-1} b_{i} \zeta^{i} T^{i}=\sum_{i \geq m}^{\beta_{k+1}-1} b_{i} \zeta^{\lambda_{i} \varepsilon_{k}} T^{i}=\sum_{i \geq m}^{\beta_{k+1}-1} b_{i} T^{i}
$$

Portanto,

$$
\varphi(T)-\varphi(\zeta T)=\left(1-\zeta^{\beta_{k+1}}\right) b_{\beta_{k+1}} T^{\beta_{k+1}}+\ldots
$$

Como $\zeta^{\beta_{k+1}} \neq 1$, pelo Lema 6.2 .3 , o resultado segue.

Vamos denotar por $f_{Y}^{j}$ a derivada parcial $\frac{\partial^{j} f}{\partial Y^{j}}$.

Teorema 6.3.2 Seja $f \in k[[X, Y]]$ irredutivel de multiplicidade $n$ e regular em $Y$. Sejam $j, k$ inteiros tais que $1 \leq j \leq n e 0 \leq k \leq g-1$. Se $e_{k+1} \leq j \leq e_{k}$, então

$$
I\left(f, f_{Y}^{j}\right)=\left(n-e_{1}\right) \beta_{1}+\left(e_{1}-e_{2}\right) \beta_{2}+\ldots+\left(e_{k-1}-e_{k}\right) \beta_{k}+\left(e_{k}-j\right) \beta_{k+1} .
$$

\section{Demonstração:}

Se $u$ é uma unidade em $k[[X, Y]]$ então

$$
I\left(u f,(u f)_{Y}\right)=I\left(u f, u_{Y} f+u f_{Y}\right)=I\left(u f, u_{Y} f+u f_{Y}-u_{Y} u^{-1} u f\right)=I\left(u f, u f_{Y}\right)=I\left(f, f_{Y}\right) .
$$

Logo podemos assumir que $f$ é um polinômio de Weierstrass. Se $\left(T^{n}, \varphi(T)\right)$ é uma parametrização de Puiseux de $(f)$, então, do Corolário 3.3.4, temos

$$
f(X, Y)=\prod_{\zeta \in G_{0}}\left(Y-\varphi\left(\zeta X^{\frac{1}{n}}\right)\right)
$$

Derivando em relação a $Y j$ vezes obtemos

$$
\begin{gathered}
f_{Y}^{j}(X, Y)=j ! \sum_{\substack{\Lambda \subset G_{0} \\
\# \Lambda=n-j}}\left[\prod_{\zeta \in \Lambda}\left(Y-\varphi\left(\zeta X^{\frac{1}{n}}\right)\right)\right] \\
\end{gathered}
$$


Então

$$
I\left(f, f_{Y^{\prime}}^{j}\right)=\operatorname{mult}\left(\sum_{\substack{\Lambda \subset G_{0} \\ \# \Lambda=n-j}}\left[\prod_{\zeta \in \Lambda}(\varphi(T)-\varphi(\zeta T))\right]\right) .
$$

Sabemos, pelo Lema 6.3.1, que a multiplicidade de $\prod_{\zeta \in \Lambda}(\varphi(T)-\varphi(\zeta T))$ depende do conjunto $G_{i} \backslash G_{i+1}$ ao qual $\zeta$ pertence e a multiplicidade será menor quanto menor for $i$, pois a sequência dos $\beta^{\prime}$ s é crescente. Como $e_{k+1} \leq j \leq e_{k}$, então $n-e_{k} \leq n-j \leq n-e_{k+1}$. Em $G_{0} \backslash G_{k+1}$ temos $n-e_{k+1}$ elementos. Comó queremos escolher $(n-j) \zeta^{\prime}$ s pertencentes aos conjuntos $G_{i} \backslash G_{i+1}$ para $i$ pequeno, então podemos escolhê-los em $G_{0} \backslash G_{k+1}$. Observemos que não podemos escolhê-los em $G_{0} \backslash G_{k}$, pois $n-j \geq n-e_{k}$. Portanto as menores multiplicidades na expressão acima vão ocorrer quando $\Lambda=\left(G_{0} \backslash G_{k}\right) \cup J$, onde $J \subset G_{k} \backslash G_{k+1}$ (ver figura abaixo).

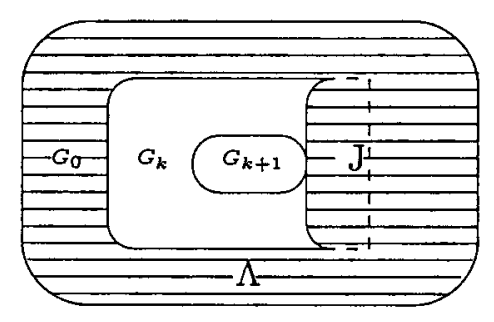

Figura 2

Assim; devemos calcular a multiplicidade da seguinte soma

$$
\begin{aligned}
& \sum_{J \subset G_{k} \backslash G_{k+1}}\left[\prod_{\zeta \in\left(G_{0} \backslash G_{k}\right) \cup J}(\varphi(T)-\varphi(\zeta T))\right]= \\
& \# J=e_{k}-j \\
& \prod_{\zeta \in G_{0} \backslash G_{k}}(\varphi(T)-\varphi(\zeta T)) \sum_{J \subset G_{k} \backslash G_{k+1}}\left[\prod_{\eta \in J}(\varphi(T)-\varphi(\eta T))\right] \\
& \# J=e_{k}-j
\end{aligned}
$$

Segue do Lema 6.3.1 que a multiplicidade do primeiro fator é

$$
\left(n-e_{1}\right) \beta_{1}+\left(e_{1}-e_{2}\right) \beta_{2}+\ldots+\left(e_{k-1}-e_{k}\right) \beta_{k}
$$

Por outro lado, cada somando do segundo fator tem multiplicidade $\left(e_{k}-j\right) \beta_{k+1}$ e coeficiente líder $b_{\beta_{k+1}}^{e_{k}-j} \prod_{\eta \in J}\left(1-\eta^{\beta_{k+1}}\right)$

Para concluir a prova devemos mostrar que

$$
\sum_{\substack{J \subset G_{k} \backslash G_{k+1} \\ \# J=e_{k}-j}}\left[\prod_{\eta \in J}\left(1-\eta^{\beta_{k+1}}\right)\right]
$$


é não nulo. Mas $\left(1-\eta^{\beta_{k+1}}\right)=0$, para todo $\eta \in G_{k+1}$, pois $e_{k+1}$ divide $\beta_{k+1}$. Logo (6.6) é igual a

$$
\sum_{\substack{J \subset G_{k} \\ \# J=e_{k}-j}}\left[\prod_{\eta \in J}\left(1-\eta^{\beta_{k+1}}\right)\right] .
$$

Observemos que, quando $\eta$ varia em $G_{k}$, a expressão $\eta^{\beta_{k+1}}$ assume $\eta_{k+1}=\frac{e_{k}}{e_{k+1}}$ valores distintos, cada um repetido $e_{k+1}$ vezes. Com isto podemos concluir que, a menos de sinal, a expressão (6.7) é o coeficiente de $Z^{j}$ do polinômio $g(Z)=\left[(1-Z)^{\eta_{k+1}}-1\right]^{e_{k+1}}$, que é, a menos de sinal, igual a

$$
\sum_{i_{1}+\ldots+i_{e_{k+1}}=j}\left(\begin{array}{c}
\eta_{k+1} \\
i_{1}
\end{array}\right) \ldots\left(\begin{array}{c}
\eta_{k+1} \\
i_{e_{k+1}}
\end{array}\right) \neq 0 .
$$

Corolário 6.3.3 Se $e_{k+1} \leq j \leq e_{k}$, então

$$
\beta_{k+1}=I\left(f, f_{Y}^{j-1}\right)-I\left(f, f_{Y}^{j}\right)
$$

\section{Demonstração:}

Do teorema acima segue que

$$
I\left(f, f_{Y}^{j-1}\right)=\left(n-e_{1}\right) \beta_{1}+\left(e_{1}-e_{2}\right) \beta_{2}+\ldots+\left(e_{k-1}-e_{k}\right) \beta_{k}+\left(e_{k}-(j-1)\right) \beta_{k+1}
$$

$\mathrm{e}$

$$
I\left(f, f_{Y}^{j}\right)=\left(n-e_{1}\right) \beta_{1}+\left(e_{1}-e_{2}\right) \beta_{2}+\ldots+\left(e_{k-1}-e_{k}\right) \beta_{k}+\left(e_{k}-j\right) \beta_{k+1} .
$$

Logo, $\beta_{k+1}=I\left(f, f_{Y}^{j-1}\right)-I\left(f, f_{Y}^{j}\right)$.

Se $f$ é regular em $X$, os resultados desta seção são válidos trocando $Y$ por $X$.

O corolário acima nos permite calcular os inteiros característicos de uma curva plana algebróide irredutível quando esta é dada na formá cartesiana. Para isto, se $f$ é regular em $Y$, é suficiente aplicar (6.8). Para $j=2>e_{g}=1$, temos que

$$
\beta_{g}=I\left(f, f_{Y}^{1}\right)-I\left(f, f_{Y}^{2}\right)
$$

Calculamos para $j=3, \ldots$ até o último valor de $j$ para o qual a diferença em (6.8) permanece a mesma. Este valor de $j$ é $e_{g-1}$. Depois calculamos para $j=e_{g-1}+1$, chegando a $\beta_{g-1}$. Novamente calculamos para $j=e_{g-1}+2, \ldots$ até o último valor de $j$ para o qual a diferença continua a mesma. Este valor de $j$ é $e_{g-2}$. Desta forma conseguimos encontrar todos os inteiros característicos de $(f)$.

Se $f$ é regular em $X$, podemos usar o algoritmo acima trocando $Y$ por $X$.

Exemplo 6.3.4 Seja $f=Y^{4}-2 X^{3} Y^{2}-4 X^{5} Y+X^{6}-X^{7}$, irredutível e regular em $Y$. Primeiramente vamos calcular os índices de interseção

$$
I\left(f, f_{Y}^{i}\right)=\text { mult } X_{X}\left(R_{Y}\left(f, f_{Y}^{i}\right)\right), i=1, \ldots, 4 .
$$

Usamos o software Maple 6 para calcular a resultante acima. O comando utilizado é resultant $\left(f, f_{Y}^{i}, Y\right)$. 
Sabendo que $f_{Y}^{1}=4 Y^{3}-4 X^{3} Y-4 X^{5}, f_{Y}^{2}=12 Y^{2}-4 X^{3}, f_{Y}^{3}=24 Y$ e $f_{Y}^{4}=24$. Obtemos $I\left(f, f_{Y}^{1}\right)=19, I\left(f, f_{Y}^{2}\right)=12, I\left(f, f_{Y}^{3}\right)=6$ e $I\left(f, f_{Y}^{4}\right)=0$.

Vamos calcular os inteiros característicos de $f$, usando o algoritmo acima. Seja $j=2>e_{g}=1$, onde $g$ é o gênero da curva $(f)$, então

$$
\beta_{g}=I\left(f, f_{Y}^{1}\right)-I\left(f, f_{Y}^{2}\right)=19-12=7 .
$$

Calculamos a diferença (6.8) para $j=3, \ldots$ até que o valor desta diferença mude. Para $j=3$, temos

$$
I\left(f, f_{Y}^{2}\right)-I\left(f, f_{Y}^{3}\right)=12-6=6 .
$$

Então $e_{g-1}=3$ e $\beta_{g-1}=6$. Para $j=e_{g-1}+1=4$, temos

$$
I\left(f, f_{Y}^{3}\right)-I\left(f, f_{Y}^{4}\right)=6-0=6 .
$$

Então

$$
\beta_{g-2}=\beta_{0}=\operatorname{mult}(f)=4
$$

Logo, $g=2, \beta_{0}=4, \beta_{1}=6$ e $\beta_{2}=7$.

\subsection{Sequência de Apéry de um semigrupo de valores}

Sejam $S$ um semigrupo com condutor c e $p \in S \backslash\{0\}$. Definimos a sequência de Apéry $a_{0}, \ldots, a_{p-1}$ de $S$ com respeito a $p$ indutivamente pelas condições

$$
a_{0}=0 \quad \text { e } \quad a_{j}=\min \left(S \backslash \bigcup_{i=0}^{j-1}\left(a_{i}+p \mathbb{N}\right)\right), 1 \leq j \leq p-1 .
$$

Se $a \in \mathbb{N}$, denotaremos por [a] a classe residual de $a$ módulo $p$ em $\mathbb{N}$.

Vejamos agora algumas propriedades das sequências de Apéry.

Proposição 6.4.1 Sejam $S$ um semigrupo com condutor e $a_{0}, \ldots, a_{p-1}$ sua sequência de Apéry. Então

(a) $a_{0}=0<a_{1}<\cdots<a_{p-1}$.

(b) $a_{i} \not \equiv a_{j} \bmod p, \quad 0 \leq i<j \leq p-1$.

(c) $a_{i}=\min \left(\left[a_{i}\right] \cap S\right)$.

(d) $S=\bigcup_{j=0}^{p-1}\left(a_{j}+p \mathbb{N}\right)$

(e) $c=a_{p-1}-(p-1)$. 


\section{Demonstração:}

(a) Observemos que $\bigcup_{i=0}^{j-1}\left(a_{i}+p \mathbb{N}\right) \subseteq \bigcup_{i=0}^{j}\left(a_{i}+p \mathbb{N}\right)$. Portanto, $S \backslash \bigcup_{i=0}^{j}\left(a_{i}+p \mathbb{N}\right) \subset S \backslash \bigcup_{i=0}^{j-1}\left(a_{i}+p \mathbb{N}\right) \mathrm{e}$ $a_{j+1}=\min \left(S \backslash \bigcup_{i=0}^{j}\left(a_{i}+p \mathbb{N}\right)\right) \geq \min \left(S \backslash \bigcup_{i=0}^{j-1}\left(a_{i}+p \mathbb{N}\right)\right)=a_{j}$

A igualdade não ocorre pois $a_{j} \in \bigcup_{i=0}^{j}\left(a_{i}+p \mathbb{N}\right)$. Logo, $a_{j}<a_{j+1}$, para todo $j=0, \ldots, p-2$.

(b) Se $i<j$, temos que $a_{j} \notin a_{i}+p \mathbb{N}$. Portanto, $a_{j} \neq a_{i}+\alpha p, \alpha \in \mathbb{N}$, e assim $a_{i} \not \equiv a_{j} \bmod p$, $0 \leq i<j \leq p-1$.

(c) Seja $\alpha \in\left[a_{i}\right] \cap S$. Logo existe $\lambda \in \mathbb{N}$ tal que $\alpha-a_{i}=\lambda p$. Portanto $\alpha \geq a_{i}$. Como $a_{i} \in\left[a_{i}\right] \cap S$, segue que $a_{i}=\min \left(\left[a_{i}\right] \cap S\right)$.

(d) Segue imediatamente da definição.

(e) Vamos mostrar que $a_{p-1}-p \notin S$ e que se $s \geq a_{p-1}-p+1$ então $s \in S$. Suponhamos que $a_{p-1}-p \in S$. Como $a_{p-1}=\min \left(\left[a_{p-1}\right] \cap S\right)$ temos que $a_{p-1}-p \notin\left[a_{p-1}\right]$, o que é uma contradição.

Seja $r$ um número natural qualquer. Suponhamos que $a_{p-1}-p+1+r \notin S$. Então existe $\lambda<0$ tal que $a_{p-1}-p+1+r=a_{i}+\lambda p$, para algum $i=0, \ldots, p-1$. Assim,

$$
a_{p-1}-a_{i}=p+\lambda p-r-1<0,
$$

o que é uma contradição.

O ítem $(d)$ da proposição acima implica que $\left\{p, a_{0}, \ldots, a_{p-1}\right\}$ gera $S$ sobre $\mathbb{N}$. Segue dos ítens $(c)$ e $(d)$ que os elementos de $S$ são da forma $a_{i}+\lambda p$, para algum $i=0, \ldots, p-1$ e $\lambda \geq 0$, enquanto que as lacunas de $S$ são da forma $a_{i}+\lambda p$, para algum $i=0, \ldots, p-1$ e $\lambda<0$.

Quando tomamos $p=n=\min (S \backslash\{0\})$ então a sequência de Apéry de $S$ com respeito a $n$ será chamada de sequência de Apéry de $S$ e o conjunto $\mathcal{A}=\left\{a_{0}, \ldots, a_{n-1}\right\}$ será chamado de conjunto de Apéry de $S$.

Seja $f \in k[[X, Y]]$ uma série de potências irredutivel regular em $Y$ de ordem $n$. Lembremos que

$$
\mathcal{O}_{f}=k[[X]]+k[[X]] y+k[[X]] y^{2}+\ldots+k[[X]] y^{n-1}
$$

e

$$
M_{k}=k[[X]]+k[[X]] y+k[[X]] y^{2}+\ldots+k[[X]] y^{k}, k=0,1, \ldots, n-1
$$

Logo

$$
k[[X]]=M_{0} \subset M_{1} \subset \ldots \subset M_{n-1}=\mathcal{O}_{f}
$$

Proposição 6.4.2 Seja $f \in k[[X, Y]]$ irredutivel regular em $Y$ de ordem $n$ e tal que $n$ não divide $m=I(f, Y)$. Sejam $M_{-1}=\{0\}$ e $y_{0}=1$. Então para todo $k=0,1, \ldots, n-1$ existe um elemento $y_{k} \in y^{k}+M_{k-1}$ tal que $v\left(y_{k}\right) \notin v\left(M_{k-1}\right)$. 


\section{Demonstração:}

Seja $\left(T^{n}, \varphi(T)\right)$ uma parametrização de Puiseux de $(f)$, onde $\varphi(T)=a_{m} T^{m}+\ldots$ Observemos que $y_{0}=1 \in y^{0}+M_{-1}=1+\{0\} \mathrm{e}$

$$
v\left(y_{0}\right)=0 \notin v\left(M_{-1}\right)=\{\infty\}
$$

Como $m$ não é múltiplo de $n$, definindo $y_{1}=y$ temos que $y_{1} \in y^{1}+M_{0}=y+k[[X]] \mathrm{e}$

$$
v\left(y_{1}\right)=v(y)=m \notin v\left(M_{0}\right)=n \mathbb{N} \text {. }
$$

Temos que $M_{1}=k[[X]]+k[[X]] y_{1}$. Consideremos o elemento $\varphi_{0}(X) \dot{+} \varphi_{1}(X) y_{1} \in M_{1}$ tal que

$$
y_{2}=y_{1}^{2}-\varphi_{0}(X)-\varphi_{1}(X) y_{1}
$$

quando visto como uma série em $T$, não contém nenhum termo de ordem múltiplo de $n$ ou de ordem $v\left(y_{1}\right)+$ múltiplo de $n$. Notemos que $v\left(y_{2}\right) \neq \infty$ pois, caso contrário, $y_{1}^{2} \in M_{1}$, o que é um absurdo. Então $y_{2} \in y^{2}+M_{1}$ e $v\left(y_{2}\right) \notin v\left(M_{1}\right)$.

Observemos que $M_{1}=k[[X]]+k[[X]] y_{1}+k[[X]] y_{2}$. Consideremos $\varphi_{0}(X)+\varphi_{1}(X) \dot{y_{1}}+\varphi_{2}(\dot{X}) y_{2} \in M_{2}$ tal que

$$
y_{3}=y_{1}^{3}-\varphi_{0}(X)-\varphi_{1}(X) y_{1}-\varphi_{2}(X) y_{2}
$$

quando visto como uma série em $T$, não contém nenhüm termo de ordem múltiplo de $n$, ou de ordem $v\left(y_{1}\right)+$ múltiplo de $n$, ou de ordem $v\left(y_{2}\right)+$ múltiplo de $n$. Notemos que $v\left(y_{3}\right) \neq \infty$ pois, caso contrário, $y_{1}^{3} \in M_{2}$, o que é um absurdo. Então $y_{3} \in y^{3}+M_{2}$ e $v\left(y_{3}\right) \notin v\left(M_{2}\right)$.

Continuando o processo, obtemos o resultado, ou seja, para todo $k=0,1, \ldots, n-1$, existe $y_{k} \in$ $y^{k}+M_{k-1}$ tal que $v\left(y_{k}\right) \notin v\left(M_{k-1}\right)$. Além disso,

$$
\mathcal{O}_{f}=k[[X]]+k[[X]] y_{1}+k[[X]] y_{2}+\ldots+k[[X]] y_{n-1} .
$$

A proposição acima mostra que $y_{0}, \ldots, y_{n-1}$ geram $\mathcal{O}_{f}$ como um $k[[X]]-$ módulo:

Lema 6.4.3 Suponhamos que exista $y_{k} \in y^{k}+M_{k-1} \operatorname{com} v\left(y_{k}\right) \notin v\left(M_{k-1}\right), k=0,1, \ldots ; n-1$, onde $y_{0}=1$ e $M_{-1}=\{0\}$. Então para todo $i, j$ com $0 \leq i, j \leq n-1$ e $i+j \leq n-1$, temos que

$$
v\left(y_{i}\right)+v\left(y_{j}\right) \leq v\left(y_{i+j}\right)
$$

\section{Demonstração:}

Escrevendo $y_{i}=a+y^{i}$ e $y_{j}=b+y^{j}$, com $a \in M_{i-1}$ e $b \in M_{j-1}$, temos

$$
y_{i} y_{j}=a b+a y^{j}+b y^{i}+y^{i+j}=c+y^{i+j}
$$

onde $c \in M_{i+j-1}$. Como $y^{i+j}=y_{i+j}-d$, para algum $d \in M_{i+j-1}$, segue que $y_{i} y_{j}=c-d+y_{i+j}$.

Se $v\left(y_{i+j}\right)<v\left(y_{i}\right)+v\left(y_{j}\right)=v\left(y_{i} y_{j}\right)$ então $v\left(y_{i+j}\right)=v(c-d) \in v\left(M_{i+j-1}\right)$, o que é uma contradição, pois $v\left(y_{i+j}\right) \notin v\left(M_{i+j-1}\right)$. 
Observação 6.4.4 Temos que $v\left(y_{i}\right)<v\left(y_{j}\right)$ sempre que $0 \leq i<j \leq n-1$. De fato, como $v\left(y_{i}\right) \notin$ $M_{i-1}$ temos que $v\left(y_{i}\right) \neq 0$. Segue do lema acima que

$$
v\left(y_{j}\right) \geq v\left(y_{i}\right)+v\left(y_{j-i}\right)>v\left(y_{i}\right) .
$$

Proposiçāo 6.4.5 Seja $f \in k[[X, Y]]$ irredutivel e regular em $Y$ de ordem $n$. Sejam $y_{0}=1$ e sejam $y_{1}, \ldots, y_{n-1}$ elementos de $\mathcal{O}_{f}$ tais que $y_{k} \in y^{k}+M_{k-1}$ e $v\left(y_{k}\right) \notin v\left(M_{k-1}\right)$. Denotando por $[r]$ a classe residual do inteiro $r$ módulo $n$ temos, para $k=0,1, \ldots, n-1$,

(i) $v\left(M_{k}\right)=\bigcup_{i=0}^{k}\left\{v\left(y_{i}\right)\right\}+n \mathbb{N}$.

(ii) $v\left(y_{i}\right) \not \equiv v\left(y_{j}\right) \bmod n$, para $i, j=0, \ldots, n-1, i \neq j$.

(iii) $v\left(y_{k}\right)=\min \left(v\left(y_{k}\right) \cap S(f)\right)$.

\section{Demonstração:}

Se $n=1$ então $S(f)=\mathbb{N}$ e $k=0$. Temos que $v\left(M_{0}\right)=v(k[[X]])=n \mathbb{N}=v\left(y_{0}\right)+n \mathbb{N}$ e $\min \left(v\left(y_{0}\right) \cap S(f)\right)=0=v\left(y_{0}\right)$, ou seja, valem as afirmações $(i)$, (ii) e (iii). Assim, podemos supor que $n>1$.

(i) A demonstração é por indução sobre $k$. Se $k=0, v\left(M_{0}\right)=v(k[[X]])=n \mathbb{N}$. Suponhamos por hipótese de indução que para algum $k, 0<k \leq n-1$,

$$
v\left(M_{k-1}\right)=\left\{v\left(y_{i}\right)+\lambda n ; 0 \leq i \leq k-1, \lambda \in \mathbb{N}\right\} .
$$

Como $M_{k}=M_{k-1}+k[[X]] y_{k}$, podemos escrever qualquer elemento $\beta \in M_{k}$ na forma $\beta=$ $\alpha+a(X) y_{k}$, com $\alpha \in M_{k-1}$ e $a(X) \in k[[X]]$. Temos que $v(\alpha) \neq v\left(a(X) y_{k}\right)$. De fato, se $v(\alpha)=v\left(a(X) y_{k}\right)$, segue da hipótese de indução que

$$
v(\alpha)=v\left(y_{i}\right)+\lambda n=v\left(a(X) y_{k}\right)=v\left(y_{k}\right)+\mu n
$$

para algum $i \leq k-1, \lambda, \mu \in \mathbb{N}$. Logo,

$$
v\left(y_{k}\right)=v\left(y_{i}\right)+(\lambda-\mu) n \text {. }
$$

Da Observação 6.4.4 temos que $\lambda>\mu$. Portanto $v\left(y_{k}\right) \in v\left(M_{k-1}\right)$, o que é um absurdo. Assim $v(\beta)=v(\alpha) \in v\left(M_{k-1}\right)$ ou $v(\beta)=v\left(a(X) y_{k}\right)=v\left(y_{k}\right)+\mu n$. Portanto, $v\left(M_{k}\right)=\bigcup_{i=0}^{k}\left\{v\left(y_{i}\right)\right\}+n \mathbb{N}$.

(ii) Suponhamos por absurdo que $v\left(y_{i}\right) \equiv v\left(y_{j}\right) \bmod n$ para algum par de inteiros $(i, j)$, com $i \neq$ $j$. Suponhamos, sem perda de generalidade, que $v\left(y_{j}\right)=v\left(y_{i}\right)+\lambda n$ e que $j>i$. Segue da Observação 6.4.4 que $\lambda>0$ e de $(i)$ temos que $v\left(y_{j}\right) \in v\left(M_{i}\right)$, o que é uma contradição.

(iii) Segue de (ii) que cada classe residual de inteiros módulo $n$ contém exatamente um dos inteiros $v\left(y_{i}\right), i=0, \ldots, n-1$. Por outro lado, os únicos elementos na classe residual de $v\left(y_{i}\right)$ são, pelo item $(i)$, os inteiros da forma $v\left(y_{i}\right)+\lambda n$, com $\lambda \geq 0$. Portanto, $v\left(y_{k}\right)=\min \left(\left[v\left(y_{k}\right)\right] \cap S(f)\right)$, $k=0, \ldots, n-1$. 
Os ítens (ii) e (iii) da proposição acima mostram que $a_{i}=v\left(y_{i}\right), i=0, \ldots, n-1$, é a sequência de Apéry de $S(f)$.

Corolário 6.4.6 Sejam $i$ e $j$ inteiros distintos: $i, j=0, \ldots, n-1$, e $\alpha_{i}(X), \alpha_{j}(X) \in k[[X]] \backslash\{0\}$. Então $v\left(\alpha_{i}(X) y_{i}\right) \not \equiv v\left(\alpha_{j}(X) y_{j}\right) \bmod n$.

\section{Demonstração:}

Temos que $v\left(\alpha_{i}(X)\right)=\lambda_{i} n$, para algum $\lambda_{i} \in \mathbb{N}$. Assumindo $j>i$, se $v\left(\alpha_{i}(X) y_{i}\right)=v\left(\alpha_{j}(X) y_{j}\right)+\alpha n$ então teríamos

$$
0<v\left(y_{j}\right)-v\left(y_{i}\right)=v\left(\alpha_{i}(X)\right)-v\left(\alpha_{j}(X)\right)+\alpha n=\left(\lambda_{i}-\lambda_{j}+\alpha\right) n,
$$

com $\lambda_{i}>\lambda_{j}$. Portanto, $v\left(y_{j}\right) \in v\left(M_{i}\right) \subset v\left(M_{j-1}\right)$, pois $i \leq j-1$, o que é uma contradição.

Corolário 6.4.7 Temos que

$$
\mathcal{O}_{f}=k[[X]] \oplus k[[X]] y_{1} \oplus k[[X]] y_{2} \oplus \ldots \oplus k[[X]] y_{n-1}
$$

\section{Demonstração:}

Sabemos que $\mathcal{O}_{f}=k[[X]]+k[[X]] y_{1}+k[[X]] y_{2}+\ldots+k[[X]] y_{n-1}$, assim basta mostrar que $y_{0}=$ $1, y_{1}, \ldots, y_{n-1}$ são linearmente independentes sobre $k[[X]]$. De fato, se existe uma relação não trivial

$$
\alpha_{0}(X)+\alpha_{1}(X) y_{1} \ldots+\alpha_{n-1}(X) y_{n-1}=0 .
$$

Então

$$
\infty=v(0)=v\left(\alpha_{0}(X)+\alpha_{1}(X) y_{1} \ldots+\alpha_{n-1}(X) y_{n-1}\right)=v\left(\alpha_{i}(X) y_{i}\right)
$$

para algum $i$ tal que $\alpha_{i}(X) \neq 0$, o que é uma contradição.

Corolário 6.4.8 Sejam $z_{0}=1, z_{1}, \ldots, z_{n-1} \in \mathcal{O}_{f}$ tais que

(i) $z_{k} \in y^{k}+M_{k-1}, k=0, \ldots, n-1, e$

(ii) $v\left(z_{0}\right), v\left(z_{1}\right), \ldots, v\left(z_{n-1}\right)$ não são dois a dois congruentes módulo $n$.

Então $v\left(z_{k}\right)=v\left(y_{k}\right)$, para todo $k=0, \ldots, n-1$.

\section{Demonstração:}

Da Proposição 6.4 .5 é suficiente mostrar que $v\left(z_{k}\right) \notin v\left(M_{k-1}\right), k=0, \ldots, n-1$, pois $(i)$ e esta condição determinam unicamente os $v\left(y_{k}\right)$. Temos que $v\left(M_{k-1}\right)=\bigcup_{i=0}^{k-1}\left\{v\left(y_{i}\right)\right\}+n \mathbb{N}$, ou seja, $v\left(M_{k-1}\right)$ intersecta apenas $k$ classes residuais módulo $n$, e como $v\left(z_{i}\right) \in v\left(M_{k-1}\right)$, para todo $i=0, \ldots, k-1$, segue que $v\left(z_{k}\right) \notin v\left(M_{k-1}\right)$, o que prova a proposição.

Observemos que o conjunto de Apéry de um semigrupo é um conjunto de geradores e, sendo assim, o conjunto de Apéry determina o conjunto minimal de geradores e vice-versa. 


\subsection{Semigrupos e blowing-ups}

Nesta seção vamos relacionar o semigrupo de um ramo plano com a sequência de multiplicidades obtida no processo de desingularização visto no Capítulo 5 .

Seja $f \in k[[X, Y]]$ uma série de potências irredutível de multiplicidade $n$ e regular em $Y$. Então, da Proposição 5.3.1, segue que $S(f) \subset S\left(f^{(1)}\right)$, onde $f^{(1)}=\sigma^{*}(f)$, pois existe um homomorfismo injetivo $\operatorname{de} \mathcal{O}_{f} \operatorname{em} \mathcal{O}_{f(1)}$.

Proposição 6.5.1 Seja $f \in k[[X, Y]]$ uma série de potências irredutivel regular em $Y$ e com cone tangente $\left(Y^{n}\right)$. Sejam $a_{0}<a_{1}<\ldots<a_{n-1}$ e $a_{0}^{\prime}<a_{1}^{\prime}<\ldots<a_{n-1}^{\prime}$, respectivamente, as sequências de Apéry de $S(f)$ e $S\left(f^{(1)}\right)$ com respeito a $n\left(n \in S(f) \subset S\left(f^{(1)}\right)\right)$. Então

$$
a_{j}=a_{j}^{\prime}+j n, j=0, \ldots, n-1 .
$$

\section{Demonstraçāo:}

Da Proposição 5.2.3 temos que $f^{(1)}$ tem cone tangente $\left(Y_{1}^{n}\right)$ e $I\left(f^{(1)}, X_{1}\right)=n$.

Temos que $\left\{a_{0}^{\prime}, a_{1}^{\prime}, \ldots, a_{n-1}^{\prime}\right\}$ é um sistema residual completo módulo $n$, ou seja,

$$
\begin{aligned}
a_{0}^{\prime} & \equiv 0 \bmod n \\
a_{1}^{\prime} & \equiv 1 \bmod n \\
\vdots & \\
a_{n-1}^{\prime} & \equiv(n-1) \bmod n .
\end{aligned}
$$

Então o conjunto $\left\{a_{0}^{\prime}, a_{1}^{\prime}+n, a_{2}^{\prime}+2 n, \ldots, a_{n-1}^{\prime}+(n-1) n\right\}$ também é um sistema residual completo módulo $n$.

Do Corolário 6.4 .8 é suficiente mostrar que existe $y_{k} \in y^{k}+M_{k-1}$ tal que $v\left(y_{k}\right)=a_{k}^{\prime}+k n$, $k=0, \ldots, n-1$.

Analogamente definimos

$$
\begin{aligned}
M_{0}^{\prime}= & k[[X]] \\
M_{1}^{\prime}= & k[[X]]+y_{1} k[[X]] \\
& \vdots \\
\mathcal{O}_{f^{(1)}} & =k[[X]]+y_{1} k[[X]]+y_{1}^{2} k[[X]]+\ldots+y_{1}^{n-1} k[[X]]
\end{aligned}
$$

onde $y_{1}=\frac{y}{X}$.

Sejam $y_{0}^{\prime}=1$ e $y_{k}^{\prime} \in\left(\frac{y}{X}\right)^{k}+M_{k-1}^{\prime}$ tal que $v\left(y_{k}^{\prime}\right)=a_{k}^{\prime}$, cuja existência em $\mathcal{O}_{f(1)}$ é assegurada por 6.4.2 e 6.4.5.

Multiplicando $y_{k}^{\prime}$ por $X^{k}, k=0, \ldots, n-1$, temos

$$
y_{k}=X^{k} y_{k}^{\prime} \in M_{k-1}+y^{k} \text {. }
$$

Portanto, $v\left(y_{k}\right)=v\left(y_{k}^{\prime}\right)+v\left(X_{k}\right)=a_{k}^{\prime}+k n$.

Quando a série de potências é regular em $X$ podemos aplicar um argumento similar trocando $\sigma$ por $\tau$.

Corolário 6.5.2 Sejam c e $c^{(1)}$ os condutores de $S(f)$ e $S\left(f^{(1)}\right)$. Então

$$
c=c^{(1)}+n(n-1) \text {. }
$$




\section{Demonstração:}

Da Proposição 6.4.1, $c=a_{n-1}-(n-1)$ e $c^{(1)}=a_{n-1}^{\prime}-(n-1)$. Além disso, $a_{n-1}=a_{n-1}^{\prime}+(n-1) n$. Logo

$$
c=a_{n-1}-(n-1)=a_{n-1}^{\prime}+(n-1) n-(n-1)=c^{(1)}+(n-1) n .
$$

Observemos que a igualdade $c^{(1)}=c-(n-1) n$ nos dá uma nova evidência de que após um número finito de blowing-ups obtemos uma curva algebróide não singular.

Corolário 6.5.3 Temos que $S\left(f^{(1)}\right)$ e mult $(f)$ determinam $S(f)$ e vice-versa.

\section{Demonstração:}

Conhecendo $S\left(f^{(1)}\right)$ temos sua sequência de Apéry com respeito a mult $(f)=n$. Usando a Proposição 6.5.1 encontramos a sequência de Apéry de $S(f)$ que determina $S(f)$, pois $S(f)=$ $\bigcup_{j=0}^{n-1}\left(a_{j}+n \mathbb{N}\right)$

Corolário 6.5.4 Dois ramos planos são equisingulares (têm mesmo semigrupo de valores) se, e somente se, eles são equisolúveis (têm mesma sequência de multiplicidades).

\section{Demonstração:}

Consideremos a resolução canônica de um ramo plano $(f)$

$$
f, f^{(1)}, \ldots, f^{(N)}
$$

Como $f^{(N)}$ é não-singular, seu semigrupo associado é $\mathbb{N}$. A sequência de Apéry de $\mathbb{N}$ em relação a $n$ é dada por $a_{i}^{(N)}=i$, então da Proposição 6.5.1 temos que a sequência de Apéry de $S\left(f^{(N-1)}\right)$ é determinada se sabemos a multiplicidade de $f^{(N-1)}$ e vice-versa. Usando indução, vemos que a sequência de multiplicidades $n^{(i)}$ de $f^{(i)}$ determina e é determinada pelo semigrupo $S(f)$.

Aplicando o Corolário 6.5.2 usado repetidas vezes obtemos uma fórmula para o condutor. Se $n^{(i)}=\operatorname{mult}\left(f^{(i)}\right)$ e $n^{(0)}=\operatorname{mult}(f)$ então

$$
\begin{gathered}
c=c^{(1)}+n^{(0)}\left(n^{(0)}-1\right) \\
c^{(1)}=c^{(2)}+n^{(1)}\left(n^{(1)}-1\right) \\
c^{(2)}=c^{(3)}+n^{(2)}\left(n^{(2)}-1\right) \\
\vdots \\
c^{(r)}=c^{(r+1)}+n^{(r)}\left(n^{(r)}-1\right)
\end{gathered}
$$

e

$$
c=\sum_{i=0}^{r} n^{(i)}\left(n^{(i)}-1\right) .
$$

Se $r+1$ for o último blowing-up então $f^{(r+1)}$ é não singular, $S\left(f^{(r+1)}\right)=\mathbb{N}$ e seu condutor é $c^{(r+1)}=0$. 
Exemplo 6.5.5 Seja $h=Y^{4}-X^{7}$ então

$$
h^{(1)}=\sigma^{*}(h)=\frac{1}{X_{1}^{4}} h\left(X_{1}, X_{1} Y_{1}\right)=\frac{1}{X_{1}^{4}}\left(X_{1}^{4} Y_{1}^{4}-X_{1}^{7}\right)=Y_{1}^{4}-X_{1}^{3} .
$$

Aplicando $\tau^{*}$ a $h^{(1)}$ obtemos

$$
h^{(2)}=\tau^{*}\left(h^{(1)}\right)=\frac{1}{Y_{2}^{3}} h^{(1)}\left(X_{2} Y_{2}, Y_{2}\right)=\frac{1}{Y_{2}^{3}}\left(Y_{2}^{4}-X_{2}^{3} Y_{2}^{3}\right)=Y_{2}-X_{2}^{3}
$$

que é não-singular. Sua sequência de multiplicidades é $(4,3,1)$. Logo,

$$
c=\sum_{i=0}^{1} n^{(i)}\left(n^{(i)}-1\right)=4(4-1)+3(3-1)=18 .
$$

Exemplo 6.5.6 Seja $f=Y^{5}-X^{8}$ então

$$
f^{(1)}=\sigma^{*}(f)=\frac{1}{X_{1}^{5}} f\left(X_{1}, X_{1} Y_{1}\right)=\frac{1}{X_{1}^{5}}\left(X_{1}^{5} Y_{1}^{5}-X_{1}^{8}\right)=Y_{1}^{5}-X_{1}^{3} .
$$

Aplicando $\tau^{*}$ a $f^{(1)}$ obtemos

$$
f^{(2)}=\tau^{*}\left(f^{(1)}\right)=\frac{1}{Y_{2}^{3}} f^{(1)}\left(X_{2} Y_{2}, Y_{2}\right)=\frac{1}{Y_{2}^{3}}\left(Y_{2}^{5}-X_{2}^{3} Y_{2}^{3}\right)=Y_{2}^{2}-X_{2}^{3} .
$$

Aplicando $\sigma^{*}$ a $f^{(2)}$-obtemos

$$
f^{(3)}=\sigma^{*}\left(f^{(2)}\right)=\frac{1}{X_{3}^{2}} f^{(2)}\left(X_{3}, X_{3} Y_{3}\right)=\frac{1}{X_{3}^{2}}\left(X_{3}^{2} Y_{3}^{2}-X_{3}^{3}\right)=Y_{3}^{2}-X_{3}
$$

que é não-singular. Sua sequência de multiplicidades é $(5,3,2,1)$. Logo,

$$
c=\sum_{i=0}^{2} n^{(i)}\left(n^{(i)}-1\right)=5(5-1)+3(3-1)+2(2-1)=28 .
$$




\section{Capítulo 7}

\section{Semigrupos dos Naturais}

Neste capítulo vamos estudar semigrupos dos números naturais e mostraremos que muitas propriedades de semigrupos de valores de ramos planos $(f)$ são consequências da inequação

$$
v_{j+1}>n_{j} v_{j}, j=1, \ldots, g-1,
$$

vista no capítulo anterior e que envolve o sistema minimal de geradores de $S(f)=\left\langle v_{0}, v_{1}, \ldots, v_{g}\right\rangle$. Lembramos que dado um semigrupo $\left\langle v_{0}, v_{1}, \ldots, v_{g}\right\rangle$ temos que $e_{i}=\operatorname{mdc}\left(v_{0}, v_{1}, \ldots, v_{i}\right), n_{i}=\frac{e_{i-1}}{e_{i}}$, $i=1, \ldots, g$, e $n_{0}=1$ são os inteiros associados a $v_{0}, v_{1}, \ldots, v_{g}$.

\subsection{Semigrupos com condutores}

Dado um semigrupo $G=\left\langle x_{0}, x_{1}, \ldots, x_{r}\right\rangle \subset \mathbb{N}$, qualquer elemento em $G$ pode ser representado de muitas maneiras na forma $\lambda_{0} x_{0}+\lambda_{1} x_{1}+\ldots+\lambda_{r} x_{r} ; \lambda_{0}, \lambda_{1}, \ldots, \lambda_{r} \in \mathbb{N}$. Por exemplo, se $G=\left\langle x_{0}, x_{1}\right\rangle=$ $\langle 3,5\rangle$ temos que $45 \in G$ pode ser escrito como

$$
45=15 x_{0}=9 x_{1}=5 x_{0}+6 x_{1} .
$$

Apesar disto, quando $G$ é um semigrupo com condutor, vamos mostrar que seus elementos podem ser representados de maneira única como uma combinação de certos elementos $x_{0}, x_{1}, \ldots, x_{r}$.

Lema 7.1.1 Sejam $x_{0}, x_{1}, \ldots, x_{r} \in \mathbb{N}$, com $\operatorname{mdc}\left(x_{0}, x_{1}, \ldots, x_{r}\right)=1, e_{i}$ e $n_{i}, i=1, \ldots, r$, seus inteiros associados. Então dado $m \in \mathbb{N}$ existem números naturais $s_{1}, \ldots, s_{r}$, únicos, tais que

$$
m=\sum_{i=1}^{r} s_{i} x_{i} \bmod x_{0}, \operatorname{com} 0 \leq s_{i}<n_{i}, i=1, \ldots, r .
$$

\section{Demonstração:}

A prova será por indução em $r$.

Se $r=1$, por hipótese, $e_{1}=\operatorname{mdc}\left(x_{0}, x_{1}\right)=1$. Segue que $n_{1}=\frac{e_{0}}{e_{1}}=x_{0}$ e $\lambda x_{0}+\mu x_{1}=1$ para algum par de inteiros $\lambda, \mu$. Então

$$
m=m \lambda x_{0}+m \mu x_{1} .
$$

Dividindo $m \mu$ por $x_{0}$, existem $q$ e $s_{1}$ tais que $0 \leq s_{1}<x_{0}=n_{1} \mathrm{e}$

$$
m \mu=q x_{0}+s_{1} .
$$


De $(*)$ e $(* *)$ temos

$$
m=m \lambda x_{0}+\left(q x_{0}+s_{1}\right) x_{1}=\left(m \lambda+q x_{1}\right) x_{0}+s_{1} x_{1} .
$$

Portanto

$$
m \equiv s_{1} x_{1} \bmod x_{0}
$$

com $0 \leq s_{1}<n_{1}$.

Suponhamos agora que o resultado seja válido para $r \geq 1$. Sejam $x_{0}, \ldots, x_{r}, x_{r+1}$ números inteiros positivos satisfazendo as hipóteses do lema. Consideremos a sequência $x_{0}^{\prime}=\frac{x_{0}}{e_{r}}, x_{1}^{\prime}=\frac{x_{1}}{e_{r}}, \ldots, x_{r}^{\prime}=\frac{x_{r}}{e_{r}}$. Como $e_{r}=\operatorname{mdc}\left(x_{0}, x_{1}, \ldots, x_{r}\right)$, então $\operatorname{mdc}\left(x_{0}^{\prime} \ldots, x_{r}^{\prime}\right)=\operatorname{mdc}\left(\frac{x_{0}}{e_{r}}, \ldots, \frac{x_{r}}{e_{r}}\right)=1$. Assim $x_{0}^{\prime}, \ldots, x_{r}^{\prime}$ satisfazem as hipóteses do lema e, por hipótese de indução, para todo inteiro $m^{\prime}$ temos que

$$
m^{\prime}=\sum_{i=1}^{r} s_{i} x_{i}^{\prime} \bmod x_{0}^{\prime}, \operatorname{com} 0 \leq s_{i}<n_{i}^{\prime}, i=1, \ldots, r,
$$

onde

$$
n_{i}^{\prime}=\frac{e_{i-1}^{\prime}}{e_{i}^{\prime}}=\frac{\operatorname{mdc}\left(x_{0}^{\prime}, \ldots, x_{i-1}^{\prime}\right)}{\operatorname{mdc}\left(x_{0}^{\prime}, \ldots, x_{i}^{\prime}\right)}=\frac{\operatorname{mdc}\left(x_{0}, \ldots, x_{i-1}\right)}{e_{r}} \frac{e_{r}}{\operatorname{mdc}\left(x_{0}, \ldots, x_{i}\right)}=\frac{e_{i-1}}{e_{i}}=n_{i}
$$

Por hipótese, existem $\lambda_{0}, \lambda_{1}, \ldots, \lambda_{r+1}$ tais que

$$
\lambda_{0} x_{0}+\lambda_{1} x_{1}+\ldots+\lambda_{r+1} x_{r+1}=1
$$

Para todo inteiro $m_{i:}$,

$$
m=\lambda_{0}^{\prime} x_{0}+\lambda_{1}^{\prime} x_{1}+\ldots+\lambda_{r+1}^{\prime} x_{r+1} .
$$

Dividindo $\lambda_{r+1}^{\prime}$ por $e_{r}$, obtemos inteiros $q$ e $s_{r+1}$, com $0 \leq s_{r+1}<e_{r}=n_{r+1}$, tais que $\lambda_{r+1}^{\prime}=q e_{r}+s_{r+1}$. Portanto, $m=\lambda_{0}^{\prime} x_{0}+\lambda_{1}^{\prime} x_{1}+\ldots+\left(q e_{r}+s_{r+1}\right) x_{r+1}$. Como $e_{r}$ divide $x_{0}, \ldots, x_{r}$ existe um inteiro $m^{\prime}$ tal que

$$
m=m^{\prime} e_{r}+s_{r+1} x_{r+1}=\left(\sum_{i=1}^{n} s_{i} x_{i}^{\prime}+\lambda x_{0}^{\prime}\right) e_{r}+s_{r+1} x_{r+1} .
$$

Como $x_{i}^{\prime} e_{r}=x_{i}, i=0,1, \ldots, r$, então

$$
m=\sum_{i=1}^{r+1} s_{i} x_{i}+\lambda x_{0}
$$

Portanto,

$$
m \equiv \sum_{i=1}^{r+1} s_{i} x_{i} \bmod x_{0}
$$

A unicidade seguirá do seguinte fato

$$
\#\left\{\sum_{i=1}^{r} s_{i} x_{i} ; 0 \leq s_{i}<n_{i}, i=1, \ldots, r\right\}=n_{1} n_{2} \ldots n_{r}=x_{0}
$$

pois existem $x_{0}$ classes residuais módulo $x_{0}$ em $\mathbb{N}$. 
Proposição 7.1.2 Sejam $x_{0}, x_{1}, \ldots, x_{r} \in \mathbb{N} \operatorname{com} \operatorname{mdc}\left(x_{0}, x_{1}, \ldots, x_{r}\right)=1$. Então

(i) Dado $m \in \mathbb{N}$ existem únicos $s_{1}, \ldots, s_{r}$ tais que

$$
m=\sum_{i=0}^{r} s_{i} x_{i} ; 0 \leq s_{i}<n_{i}, i=1, \ldots r, s_{0} \in \mathbb{Z} .
$$

(ii) Se $m>\sum_{i=0}^{r}\left(n_{i}-1\right) x_{i}-x_{0}$, então $s_{0} \geq 0$.

\section{Demonstração:}

(i) Segue diretamente do Lema 7.1.1.

(ii) Suponhamos que $m>\sum_{i=0}^{r}\left(n_{i}-1\right) x_{i}-x_{0}$. Por (i) temos

$$
m=\sum_{i=0}^{r} s_{i} x_{i} ; 0 \leq s_{i}<n_{i}, i=1, \ldots, r, s_{0} \in \mathbb{Z} .
$$

Assim

$$
\sum_{i=0}^{r} s_{i} x_{i}=m>\sum_{i=0}^{r}\left(n_{i}-1\right) x_{i}-x_{0}=\sum_{i=1}^{r}\left(n_{i}-1\right) x_{i}-x_{0} \geq \sum_{i=1}^{r} s_{i} x_{i}-x_{0},
$$

donde $s_{0} x_{0}>-x_{0}$ e, portanto, $s_{0} \geq 0$.

Vimos na proposição acima que todo natural maior que $\sum_{i=1}^{r}\left(n_{i}-1\right) x_{i}-x_{0}$ é combinação linear de $x_{0}, x_{1}, \ldots, x_{r}$. Portanto, temos o seguinte:

Corolário 7.1.3 Sejam $G$ um semigrupo em $\mathbb{N}$ com condutor $c$ e $\left\{x_{0}, x_{1}, \ldots, x_{r}\right\}$ um conjunto de geradores de $G$. Então

$$
c \leq \sum_{i=1}^{r}\left(n_{i}-1\right) x_{i}-x_{0}+1 .
$$

Observemos que, na Proposição 7.1.2, se $s_{0} \geq 0$ então $m \in G$. Mas a recíproca não é sempre válida, isto é, podemos ter $m \in G$ com $s_{0}<0$, como veremos no exemplo a seguir.

\section{Exemplo 7.1.4 Seja}

$$
G=\langle 8,10,11\rangle=\{0,8,10,11,16,18,19,20,21,22,24,26,27,28,29,30,31,32,33, \ldots\} .
$$

Neste caso $e_{0}=8, e_{1}=\operatorname{mdc}(8,10)=2$ e $e_{2}=\operatorname{mdc}(8,10,11)=1$. Temos que $22 \in G$ e

$$
22=-1.8+3.10+0.11 \text {. }
$$

Assim, $s_{0}=-1<0, s_{1}=3<n_{1}=\frac{e_{0}}{e_{1}}=4$ e $s_{2}=0<n_{2}=\frac{e_{1}}{e_{2}}=2$.

Observemos que a estimativa para o condutor $c$ dada pelo Corolário 7.1.3 é

$$
c \leq \sum_{i=1}^{2}\left(n_{i}-1\right) x_{i}-x_{0}+1=(4-1) 10+(2-1) 11-8+1=34
$$

e que o condutor deste semigrupo é 26 . 
Existe uma importante classe de semigrupos em $\mathbb{N}$ na qual vale a recíproca do item (ii) da Proposição 7.1.2. Para definir esta classe de semigrupos vamos precisar da seguinte definição:

Definição 7.1.5 Seja $x_{0}, x_{1}, \ldots, x_{r}$ uma sequência de inteiros tais que mdc $\left(x_{0}, x_{1}, \ldots, x_{r}\right)=1 e$ $n_{0}, n_{1}, \ldots, n_{r}$ seus inteiros associados. Dizemos que a sequência é boa se

$$
n_{i} x_{i} \in\left\langle x_{0}, \ldots, x_{i-1}\right\rangle, \quad i=1, \ldots, r .
$$

Proposição 7.1.6 Seja $x_{0}, x_{1}, \ldots, x_{r}$ uma sequência boa de inteiros. Se $G=\left\langle x_{0}, x_{1}, \ldots, x_{r}\right\rangle$ então

(i) Se $m \in G$, existem únicos $s_{0}, \ldots, s_{r}$ tais que

$$
m=\sum_{i=0}^{r} s_{i} x_{i} ; 0 \leq s_{i}<n_{i}, i=1, \ldots, r, s_{0} \in \mathbb{N} .
$$

(ii) $O$ condutor c de $G$ é dado por

$$
c=\sum_{i=1}^{r}\left(n_{i}-1\right) x_{i}-x_{0}+1
$$

\section{Demonstração:}

(i) Seja $m=\lambda_{0} x_{0}+\lambda_{1} x_{1}+\ldots+\lambda_{r} x_{r} \in G$, com $\lambda_{i} \in \mathbb{N}$ e escreva $\lambda_{r}=q_{r} n_{r}+s_{r}$, com $0 \leq s_{r}<n_{r}$. Então

$$
m=\lambda_{0} x_{0}+\lambda_{1} x_{1}+\ldots+q_{r} n_{r} x_{r}+s_{r} x_{r} .
$$

Como a sequência é boa, $n_{r} x_{r} \in\left\langle x_{0}, \ldots, x_{r-1}\right\rangle$. Logo,

$$
\lambda_{0} x_{0}+\lambda_{1} x_{1}+\ldots+\lambda_{r-1} x_{r-1}+q_{r} n_{r} x_{r} \in\left\langle x_{0}, \ldots, x_{r-1}\right\rangle .
$$

Portanto, existem $\beta_{0}, \ldots, \beta_{r-1} \in \mathbb{N}$ tais que

$$
m=\beta_{0} x_{0}+\beta_{1} x_{1}+\ldots+\beta_{r-1} x_{r-1}+s_{r} x_{r} .
$$

Dividindo $\beta_{r-1}$ por $n_{r-1}$ temos $\beta_{r-1}=q_{r-1} n_{r-1}+s_{r-1}$, com $0 \leq s_{r-1}<n_{r-1}$. Como a sequência é boa, $n_{r-1} x_{r-1} \in\left\langle x_{0}, \ldots, x_{r-2}\right\rangle$. Logo,

$$
\beta_{0} x_{0}+\beta_{1} x_{1}+\ldots+\beta_{r-2} x_{r-2}+q_{r-1} n_{r-1} x_{r-1} \in\left\langle x_{0}, \ldots, x_{r-2}\right\rangle .
$$

Portanto, existem $\lambda_{0}, \ldots, \lambda_{r-2} \in \mathbb{N}$ tais que

$$
m=\gamma_{0} x_{0}+\gamma_{1} x_{1}+\ldots+\gamma_{r-2} x_{r-2}+s_{r-1} x_{r-1}+s_{r} x_{r} .
$$

Continuando o processo, obtemos

$$
m=\sum_{i=0}^{r} s_{i} x_{i} ; 0 \leq s_{i}<n_{i}, i=1, \ldots, r, s_{0} \in \mathbb{N} .
$$


(ii) Do Corolário 7.1.3 sabemos que

$$
c \leq \sum_{i=1}^{r}\left(n_{i}-1\right) x_{i}-x_{0}+1 .
$$

Do item (i) temos que $\sum_{i=1}^{r}\left(n_{i}-1\right) x_{i}-x_{0} \notin G$. Logo

$$
c=\sum_{i=1}^{r}\left(n_{i}-1\right) x_{i}-x_{0}+1 .
$$

É possível mostrar que a recíproca da Proposição 7.1.6 é verdadeira (ver [11]).

Definição 7.1.7 Um semigrupo $G$ em $\mathbb{N}$ com condutor c será chamado simétrico se, para todo $z \in \mathbb{Z}$, $z \in G$ se, e somente se, $c-1-z \notin G$.

Proposição 7.1.8 Seja $G$ um semigrupo em $\mathbb{N}$ com condutor $c$. As seguintes afirmações são equivalentes

(i) G é simétrico.

(ii) $2|G \cap[0, c)|=c$.

(iii) $2|\mathbb{N} \backslash G|=c$.

(iv) $|\mathbb{N} \backslash G|=|G \cap[0, c)|$.

\section{Demonstração:}

Temos que $(\mathbb{N} \backslash G) \cup(G \cap[0, c))=[0, c) \cap \mathbb{N}$. Portanto,

$$
|\mathbb{N} \backslash G|+|G \cap[0, c)|=c .
$$

Se $2|G \cap[0, c)|=c$ então $|G \cap[0, c)|=\frac{c}{2}$. De $(*)$, segue que $|\mathbb{N} \backslash G|=\frac{c}{2}$. Logo, $2|\mathbb{N} \backslash G|=c$.

Se $2|\mathbb{N} \backslash G|=c$, segue de $(*)$ que $|\mathbb{N} \backslash G|+|G \cap[0, c)|=2|\mathbb{N} \backslash G|$. Logo, $|G \cap[0, c)|=|\mathbb{N} \backslash G|$.

Se $|\mathbb{N} \backslash G|=|G \cap[0, c)|$ então, de $(*)$, temos $|G \cap[0, c)|+|G \cap[0, c)|=2|G \cap[0, c)|=c$.

Assim, $(i i),(i i i)$ e (iv) são equivalentes. Mostremos agora que (i) é equivalente a (iv). Consideremos a bijeção

$$
\begin{aligned}
\varphi:[0, c-1] \cap \mathbb{N} & \longrightarrow[0, c-1] \cap \mathbb{N} \\
z & \longmapsto c-z-1
\end{aligned}
$$

Observemos que se $0 \leq z \leq c-1$ então $c-1+1-c=0 \leq c-1-z \leq c-1$, logo $\varphi$ está bem definida. Temos que $\varphi$ é sobre pois se $d \in[0, c-1] \cap \mathbb{N}$ então $d=c-1-z$, par algum $z \in[0, c-1] \cap \mathbb{N}$. Assim, $z=c-1-d$ é tal que $\varphi(z)=d$. Além disso, $\varphi$ é claramente injetora.

Como $c$ é condutor de $G, c-1 \notin G$. Afrrmamos que $G$ é simétrico se, e somente se, $\varphi(G \cap[0, c-1])=$ $\mathbb{N} \backslash G$. De fato, suponhamos $G$ é simétrico. Então, $\varphi(G \cap[0, c-1]) \subset \mathbb{N} \backslash G$. Se $n \in \mathbb{N} \backslash G$, então $n \leq c-1$ e $\varphi(n)=c-1-n \in G$, pois $c-1-(c-1-n)=n \notin G$. Assim, $\mathbb{N} \backslash G \subset \varphi(G \cap[0, c-1])$ e a igualdade segue. Suponhamos agora que $\varphi(G \cap[0, c-1])=\mathbb{N} \backslash G$. Seja $z \in G$. Se $z \geq c$ então $c-1-z \leq-1 \notin G$. Se $z<c-1$ então $\varphi(z)=c-1-z \notin G$. Assim, $G$ simétrico. 
Portanto, (i) é equivalente a (iv).

Se $G$ é simétrico, então $c$ é par e existe o mesmo número de lacunas e de elementos de $G$ no intervalo $[0, c)$.

Proposição 7.1.9 Todo semigrupo gerado por um sequência boa é simétrico.

\section{Demonstração:}

Seja $x_{0}, x_{1}, \ldots, x_{r}$ uma sequência boa. Segue da Proposição 7.1.6 que o condutor $c$ do semigrupo $G=\left\langle x_{0}, x_{1}, \ldots, x_{r}\right\rangle$ é dado por

$$
c=\sum_{i=1}^{r}\left(n_{i}-1\right) x_{i}-x_{0}+1 .
$$

Da Proposição 7.1.2(i) sabemos que todo número natural $z$ tem sua representação única

$$
z=\sum_{i=0}^{r} s_{i} x_{i} ; 0 \leq s_{i}<n_{i}, i=1, \ldots, r, s_{0} \in \mathbb{Z} .
$$

Consideremos

$$
c-1-z=\sum_{i=1}^{r}\left(n_{i}-1\right) x_{i}-x_{0}-\sum_{i=0}^{r} s_{i} x_{i}=\sum_{i=1}^{r}\left(n_{i}-1-s_{i}\right) x_{i}-x_{0}\left(1+s_{0}\right) .
$$

Segue da Proposição 7.1.6(i) que $z \in G$ se, e somente se, $s_{0} \geq 0$ se, e somente se, $-\left(1+s_{0}\right) \leq-1$, se, e somente se, $c-1-z \notin G$.

\subsection{Semigrupos fortemente crescentes}

Seja $x_{0}, x_{1}, \ldots, x_{r}$ uma sequência de naturais tais que mdc $\left(x_{0}, x_{1}, \ldots, x_{r}\right)=1, e_{i}$ e $n_{i}, i=0, \ldots, r$, seus inteiros associados. Lembramos que uma sequência é fortemente crescente se

$$
x_{i}>n_{i-1} x_{i-1}, \quad i=1, \ldots, r \text {. }
$$

Toda sequência fortemente crescente é uma sequência crescente. De fato,

$$
x_{1}>n_{0} x_{0}=x_{0} \text { e } x_{i+1}>n_{i} x_{i}=\frac{e_{i-1}}{e_{i}} x_{i}>x_{i}, i=1, \ldots, r-1 .
$$

Lema 7.2.1 Seja $x_{0}, x_{1}, \ldots, x_{r}$ uma sequência fortemente crescente de números naturais. Então

$$
x_{i+1}>\sum_{j=0}^{i}\left(n_{j}-1\right) x_{j}, \quad i=0, \ldots, r-1 .
$$

\section{Demonstração:}

A prova será por indução sobre $i$.

Se $i=0$ temos

$$
x_{1}>\left(n_{0}-1\right) x_{0}=0 .
$$


Suponhamos que a desigualdade é válida para $i-1$, ou seja,

$$
x_{i}>\sum_{j=0}^{i-1}\left(n_{j}-1\right) x_{j}
$$

Como a sequência é fortemente crescente, temos que

$$
x_{i+1}>n_{i} x_{i}=\left(n_{i}-1\right) x_{i}+x_{i}>\left(n_{i}-1\right) x_{i}+\sum_{j=0}^{i-1}\left(n_{j}-1\right) x_{j}=\sum_{j=0}^{i}\left(n_{j}-1\right) x_{j} .
$$

Proposição 7.2.2 Toda sequência fortemente crescente de naturais ë boa.

\section{Demonstração:}

Seja $x_{0}, x_{1}, \ldots, x_{r}$ uma sequência fortemente crescente de números naturais. Do Lema 7.2.1 temos que $x_{i+1}>\sum_{j=0}^{i}\left(n_{j}-1\right) x_{j}, i=0, \ldots, r-1$. Como $n_{i} \geq 1, i=0, \ldots, r$, então

$$
n_{i+1} x_{i+1} \geq x_{i+1}>\sum_{j=0}^{i}\left(n_{j}-1\right) x_{j}>\sum_{j=0}^{i}\left(n_{j}-1\right) x_{j}-x_{0} .
$$

Mas sabemos, do Corolário 7.1.3, que o condutor $c$ do semigrupo gerado por $x_{0}, x_{1}, \ldots, x_{i}$ é tal que

$$
c \leq \sum_{j=1}^{i}\left(n_{j}-1\right) x_{j}-x_{0}+1
$$

Logo, $n_{i+1} x_{i+1} \in\left\langle x_{0}, x_{1}, \ldots, x_{i}\right\rangle, i=0, \ldots, r-1, \mathrm{e}$, portanto, a sequência é boa.

Segue da Proposição 7.1.9 que toda sequência fortemente crescente gera um semigrupo simétrico.

Definição 7.2.3 Dizemos que um semigrupo é fortemente crescente se seu sistema minimal dé geradores é fortemente crescente.

Sejam $x, y \in \mathbb{N}^{r}$. Dizemos que $x$ é menor que $y$ na ordem lexicográfica reversa, escrevendo $x \prec y$, se a última coordenada não nula de $y-x$ é positiva. Isto estabelece uma relação de ordem total em $\mathbb{N}^{r}$.

Seja $x_{0}, x_{1}, \ldots, x_{r}$ uma sequência de naturais tais que mdc $\left(x_{0}, \ldots, x_{r}\right)=1$. Definimos

$$
E\left(x_{0}, \ldots, x_{r}\right)=\left\{\left(s_{1}, \ldots, s_{r}\right) \in \mathbb{N}^{r} ; 0 \leq s_{i}<n_{i}, i=1, \ldots, r\right\} \subset \mathbb{N}^{r} .
$$

Vamos considerar $E\left(x_{0}, \ldots, x_{r}\right)$ com a ordem lexicográfica reversa e $\mathbb{N}$ com sua ordem usual.

Lema 7.2.4 Se $x_{0}, x_{1}, \ldots, x_{r}$ é uma sequência fortemente crescenté então a aplicação

$$
\begin{aligned}
\zeta: E\left(x_{0}, \ldots, x_{r}\right) & \longrightarrow \mathbb{N} \\
\left(s_{1}, \ldots, s_{r}\right) & \longmapsto \sum_{i=1}^{r} s_{i} x_{i}
\end{aligned}
$$

preserva ordem. 


\section{Demonstração:}

Se $s_{i}<n_{i}, i=1, \ldots, j$, então

$$
\sum_{i=1}^{j} s_{i} x_{i}<x_{j+1}, j=1, \ldots, r-1
$$

De fato, do Lema 7.2.1, temos

$$
x_{j+1}>\sum_{i=0}^{j}\left(n_{i}-1\right) x_{i}=\sum_{i=1}^{j}\left(n_{i}-1\right) x_{i}, \quad j=0, \ldots, r-1 .
$$

Se $s_{i}<n_{i}, i=1, \ldots, j$, então $s_{i} \leq n_{i}-1, i=1, \ldots, j_{\text {: e }}$

$$
x_{j+1}>\sum_{i=1}^{j} s_{i} x_{i}
$$

Seja $\left(s_{1}, \ldots, s_{r}\right) \prec\left(t_{1}, \ldots, t_{r}\right)$, ou seja, a última coordenada não nula de $\left(t_{1}-s_{1}, \ldots, t_{r}-s_{r}\right)$ é positiva. Queremos mostrar que $\sum_{i=1}^{r} s_{i} x_{i}<\sum_{i=1}^{r} t_{i} x_{i}$. Suponhamos, sem perda de generalidade, que $t_{r}-s_{r}>0$. Então

$$
\begin{gathered}
\sum_{i=1}^{r}\left(s_{i}-t_{i}\right) x_{i}=\left(s_{1}-t_{1}\right) x_{1}+\ldots+\left(s_{r-1}-t_{r-1}\right) x_{r-1}+\left(s_{r}-t_{r}\right) x_{r} \leq \\
\left(s_{1}-t_{1}\right) x_{1}+\ldots+\left(s_{r-1}-t_{r-1}\right) x_{r-1}-x_{r}<x_{r}-x_{r}=0
\end{gathered}
$$

pois $\left(s_{i}-t_{i}\right)<n_{i}, i=1, \ldots, r$.

Portanto, a aplicação $\zeta$ preserva ordem.

\subsection{Sequências de Apéry}

Sejam $S$ um semigrupo com condutor $c$ e $n$ sua multiplicidade. O conjunto de Apéry $\left\{a_{0}, \ldots, a_{n-1}\right\}$ de $S$ é dado por

$$
a_{0}=0 \text { e } a_{j}=\min \left(S \backslash \bigcup_{i=0}^{j-1}\left(a_{i}+n \mathbb{N}\right)\right), 1 \leq j \leq n-1
$$

Proposição 7.3.1 Seja $S=\left\langle x_{0}, \ldots, x_{r}\right\rangle$ onde $x_{0}, \ldots, x_{r}$ é uma sequência boa. Consideremos

$$
\mathcal{A}=\left\{\sum_{i=1}^{r} s_{i} x_{i} ; 0 \leq s_{i}<n_{i}, i=1, \ldots, r\right\}
$$

onde $n_{1}, \ldots, n_{r}$ são os inteiros associados a $x_{0}, \ldots, x_{r}$. Então $\mathcal{A}$ é o conjunto de Apéry de $S$. 


\section{Demonstração:}

Como a sequência $x_{0}, \ldots, x_{r}$ é boa segue, da Proposição 7.1.6, que todo $m \in S$ pode ser escrito de forma única como

$$
m=\sum_{i=1}^{r} s_{i} x_{i}+s_{0} x_{0} ; 0 \leq s_{i}<n_{i}, i=1, \ldots, r, s_{0} \in \mathbb{N}
$$

Isto implica que cada soma $\sum_{i=1}^{r} s_{i} x_{i}$ é minimal em sua classe módulo $n=x_{0}$, logo

$$
\left\{\sum_{i=1}^{r} s_{i} x_{i} ; \quad 0 \leq s_{i}<n_{i}, i=1, \ldots, r\right\} \subset \mathcal{A}
$$

pois $a_{i}=\min \left(\left[a_{i}\right] \cap S\right)$.

Como \#\{ $\left.\sum_{i=1}^{r} s_{i} x_{i} ; 0 \leq s_{i}<n_{i}, i=1, \ldots, r\right\}=n_{1} \ldots n_{r}=n$, segue que

$$
\mathcal{A}=\left\{\sum_{i={ }^{\top}}^{r} s_{i} x_{i} ; \quad 0 \leq s_{i}<n_{i}, i=1, \ldots, r\right\}
$$

Lema 7.3.2 Seja $x_{0}, x_{1}, \ldots, x_{r}$ uma sequência de números naturais tal que $m d c\left(x_{0}, x_{1}, \ldots, x_{r}\right)=1$. A aplicação

$$
\begin{aligned}
\lambda: E\left(x_{0}, \ldots, x_{r}\right) & \longrightarrow \mathbb{N} \\
\left(s_{1}, \ldots, s_{r}\right) & \longmapsto \sum_{i=1}^{r} s_{i} n_{0} \ldots n_{i-1}
\end{aligned}
$$

preserva ordem e é uma bijeção entre $E\left(x_{0}, \ldots, x_{r}\right)$ e $\{0,1, \ldots, n-1\}$, onde $n=n_{1} \ldots n_{r}$.

\section{Demonstração:}

Se $s_{i}<n_{i}$ então $s_{i} \leq n_{i}-1$ e

$$
\sum_{i=1}^{j} s_{i} n_{0} \ldots n_{i-1}=s_{1} n_{0}+s_{2} n_{0} n_{1}+\ldots+s_{j} n_{0} \ldots n_{j-1} \leq
$$

$$
\left(n_{1}-1\right) n_{0}+\left(n_{2}-1\right) n_{0} n_{1}+\ldots+\left(n_{j}-1\right) n_{0} \ldots n_{j-1}=n_{0} \ldots n_{j}-n_{0}=n_{0} \ldots n_{j}-1<n_{0} \ldots n_{j},
$$

para todo $j=1, \ldots, r$.

As desigualdades acima mostram que $\lambda$ preserva ordem. De fato, se $\left(s_{1}, \ldots, s_{r}\right) \prec\left(t_{1}, \ldots, t_{r}\right)$ em $E\left(x_{0}, \ldots, x_{r}\right)$, ou seja, a última coordenada não nula de $\left(t_{1}-s_{1}, \ldots, t_{r}-s_{r}\right)$ é positiva, digamos $t_{r}-s_{r}>0$, temos que

$$
\begin{gathered}
\sum_{i=1}^{r} s_{i} n_{0} \ldots n_{i-1}-\sum_{i=1}^{r} t_{i} n_{0} \ldots n_{i-1}=\left(s_{1}-t_{1}\right) n_{0}+\left(s_{2}-t_{2}\right) n_{0} n_{1}+\ldots+\left(s_{r}-t_{r}\right) n_{0} \ldots n_{r-1}< \\
n_{0} \ldots n_{r-1}-n_{0} \ldots n_{r-1}=0
\end{gathered}
$$

pois, $s_{i}-t_{i}<n_{i}, i=1, \ldots, r-1$, e $s_{r}-t_{r} \leq-1$. Portanto, $\lambda$ preserva ordem. 
A imagem de $\lambda$ está contida no conjunto $\{0,1, \ldots, n-1\}$, pois $\sum_{i=1}^{r} s_{i} n_{0} \ldots n_{i-1}<n_{0} \ldots n_{r}=n$. Como $\lambda$ preserva ordem segue que ela é injetiva. Além disso $E\left(x_{0}, \ldots, x_{r}\right)$ e $\{0,1, \ldots, n-1\}$ tem a mesma cardinalidade. Portanto, $\lambda$ é uma bijeção em $\{0,1, \ldots, n-1\}$.

Já sabemos que $\mathcal{A}=\left\{\sum_{i=1}^{r} s_{i} x_{i} ; 0 \leq s_{i}<n_{i}, i=1, \ldots, r\right\}$, mas queremos saber qual $a_{j}$ corresponde a uma dada soma $\sum_{i=1}^{r} s_{i} x_{i}$ e qual elemento do conjunto de Apéry corresponde a um dado gerador $x_{i}$. Saberemos responder esta questão quando o semigrupo é fortemente crescente.

Teorema 7.3.3 Seja $x_{0}, \ldots, x_{r}$ uma sequência fortemente crescente dé naturais e $a_{0}, \ldots, a_{n-1}$, onde $n=x_{0}$, o conjunto de Apéry de $S=\left\langle x_{0}, \ldots, x_{r}\right\rangle$. Então

(i) $S e\left(s_{1}, \ldots, s_{r}\right) \in E\left(x_{0}, \ldots, x_{r}\right)$ então

$$
a_{\sum_{i=1}^{r} s_{i} n_{0} \ldots n_{i-1}}=\sum_{i=1}^{r} s_{i} v_{i}
$$

(ii) Para todo $i=1, \ldots, r$, temos que

$$
x_{i}=a_{\frac{n}{e_{i-1}}}
$$

\section{Demonstração:}

(i) Segue dos Lemas 7.2.4 e 7.3.2 que a aplicação $\zeta \circ \lambda^{-1}$ dada por

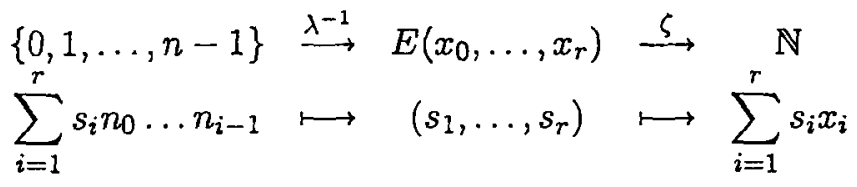

preserva ordem.

Como $\lambda$ é uma bijeção e $E\left(x_{0}, \ldots, x_{r}\right)$ tem $n$ elementos, existem $n$ índices do tipo $\sum_{i=1}^{r} s_{i} n_{0} \ldots n_{i-1}$. Então

$$
a_{\sum_{i=1}^{r} s_{i} n_{0} \ldots n_{i-1}}=\sum_{i=1}^{r} s_{i} x_{i}
$$

(ii) $\operatorname{Se}\left(s_{1}, \ldots, s_{r}\right)=(0, \ldots, \underbrace{1}, \ldots, 0)$ então

i-ésima

posição

$$
\sum_{i=1}^{r} s_{i} x_{i}=x_{i}=a_{n_{0} \ldots n_{i-1}}=a_{\frac{n}{e_{i-1}}}
$$




\subsection{Aplicação ao semigrupos de valores}

Vamos agora aplicar as propriedades de semigrupos dos naturais vistas até agora ao semigrupo de valores de um ramo plano. Como o semigrupo de valores $S(f)$ de um ramo plano $(f)$ é fortemente crescente, seu sistema minimal de geradores $x_{0}, \ldots, x_{r}$ é uma sequência boa (ver Proposição 7.2.2). Da Proposição 7.1.6 o condutor de $S(f)$ é dado por

$$
c=\sum_{i=1}^{r}\left(n_{i}-1\right) x_{i}-x_{0}+1
$$

e da Proposição 7.2.2 juntamente com a Proposição 7.1.9, temos que $S(f)$ é um semigrupo simétrico. Da Proposição 7.1.8 segue que $c$ é um natural par.

Proposiçāo 7.4.1 Seja $(f)$ um ramo plano e $x_{0}, \ldots, x_{r}$ o sistema minimal de geradores de $S(f)$. Se c é o condutor de $S(f)$ então

$$
c=n_{r} x_{r}-\beta_{r}-x_{0}+1
$$

\section{Demonstração:}

Da Proposição 7.1.6 temos que

$$
\begin{gathered}
c=\sum_{i=1}^{r}\left(n_{i}-1\right) x_{i}-x_{0}+1=\left(n_{1}-1\right) x_{1}+\left(n_{2}-1\right) x_{2}+\ldots+\left(n_{r}-1\right) x_{r}-x_{0}+1= \\
n_{r} x_{r}-\left[x_{r}-\left(n_{r-1}-1\right) x_{r-1}-\ldots-\left(n_{1}-1\right) x_{1}\right]-x_{0}+1 .
\end{gathered}
$$

Mas $\beta_{r}=x_{r}-\left(n_{r-1}-1\right) x_{r-1}-\ldots-\left(n_{1}-1\right) x_{1}(\operatorname{ver}(6.4))$, logo

$$
c=n_{r} x_{r}-\beta_{r}-x_{0}+1 \text {. }
$$

Corolário 7.4.2 Seja $(f)$ uma curva algebróide irredutível plana. Se $x_{0}, \ldots, x_{r .}$ é o șistema minimal de geradores de $S(f)$ e c é o seu condutor, então

$$
c=\sum_{i=1}^{r}\left(e_{i-1}-e_{i}\right) \beta_{i}-\beta_{0}+1 .
$$

\section{Demonstração:}

De (6.3) temos que

$$
x_{r}=\left(n_{1}-1\right) n_{2} \ldots n_{r-1} \beta_{1}+\left(n_{2}-1\right) n_{3} \ldots n_{r-1} \beta_{2}+\ldots+\left(n_{r-1}-1\right) \beta_{r-1}+\beta_{r} .
$$

Da Proposição 7.4.1 temos

$$
c=n_{r} x_{r}-\beta_{r}-x_{0}+1
$$

Então

$$
c=\left(n_{1}-1\right) n_{2} \ldots n_{r} \beta_{1}+\left(n_{2}-1\right) n_{3} \ldots n_{r} \beta_{2}+\ldots+\left(n_{r-1}-1\right) n_{r} \beta_{r-1}+n_{r} \beta_{r}-\beta_{r}-x_{0}+1
$$




$$
c=\left(e_{0}-e_{1}\right) \beta_{1}+\left(e_{1}-e_{2}\right) \beta_{2}+\ldots+\left(e_{r-2}-e_{r-1}\right) \beta_{r-1}+n_{r} \beta_{r}-\beta_{r}-x_{0}+1,
$$

onde $n_{r}=e_{r-1}, e_{r}=1$ e $x_{0}=\beta_{0}$. Logo,

$$
c=\sum_{i=1}^{r}\left(e_{i-1}-e_{i}\right) \beta_{i}-\beta_{0}+1 .
$$

Corolário 7.4.3 Sejam $f \in \mathcal{M} \subset k[[X, Y]]$ uma série de potências irredutível, $n=I(f, X)$ e $m=$ $I(f, Y)$. Se c é o condutor de $S(f)$ então $I\left(f, f_{Y}\right)=c+n-1$ e $I\left(f, f_{X}\right)=c+m-1$.

\section{Demonstração:}

Suponhamos que $f$ é regular em $Y$. Aplicando o Teorema 6.3.2 para $j=1$ e $k=r-1$, obtemos

$$
I\left(f, f_{Y}\right)=\left(n-e_{1}\right) \beta_{1}+\left(e_{1}-e_{2}\right) \beta_{2}+\ldots+\left(e_{r-2}-e_{r-1}\right) \beta_{r-1}+\left(e_{r-1}-1\right) \beta_{r}
$$

que é igual a $c+n-1$ pelo Corolário 7.4.2.

Seja $(x(T), y(T))$ uma parametrização primitiva de $(f)$. Aplicando a Regra da Cadeia, obtemos

$$
f_{X}(x(T), \dot{y}(T)) x^{\prime}(T)+f_{Y}(x(T), y(T)) y^{\prime}(T)=0 .
$$

Portanto,

$$
\begin{gathered}
I\left(f, f_{X}\right)=\operatorname{mult}\left(f_{X}(x(T), y(T))\right)=\operatorname{mult}\left(f_{Y}(x(T), y(T)) y^{\prime}(T)\right)-\operatorname{mult}\left(x^{\prime}(T)\right) \\
=c+n-1+m-1-(n-1)=c+m-1 .
\end{gathered}
$$

Em [12] J.Milnor introduziu o seguinte número

$$
\mu_{f}:=\operatorname{dim}_{k} \frac{k[[X, Y]]}{\left(f_{X}, f_{Y}\right)}=I\left(f_{X}, f_{Y}\right)
$$

que é conhecido como o número de Milnor de $f$.

O Teorema a seguir, provado por Milnor usando métodos topológicos, foi provado algebricamente por J.J.Risler em [13].

Vamos precisar da seguinte proposição:

Proposição 7.4.4 Seja $f \in \mathcal{M} \subset k[[X, Y]]$ irredutivel, de multiplicidade $n$ e regular em $Y$. Então

$$
I\left(f^{(1)},\left(f^{(1)}\right)_{Y_{1}}\right)=I\left(n f^{(1)}+X_{1}\left(f^{(1)}\right)_{X_{1}},\left(f^{(1)}\right)_{Y_{1}}\right),
$$

onde $f^{(1)}\left(X_{1}, Y_{1}\right)=\frac{1}{X_{1}^{n}} f\left(X_{1}, X_{1} Y_{1}\right)$.

\section{Demonstração:}

Suponhamos $\left(f^{(1)}\right)_{Y_{1}}=p_{1}^{\alpha_{1}} \ldots p_{r}^{\alpha_{r}}$, onde cada $p_{i}$ é uma série de potências irredutível. Então.

$$
I\left(f^{(1)},\left(f^{(1)}\right)_{Y_{1}}\right)=\sum_{i=1}^{r} \alpha_{i} I\left(f^{(1)}, p_{i}\right)
$$


e

$$
I\left(n f^{(1)}+X_{1}\left(f^{(1)}\right)_{X_{1}},\left(f^{(1)}\right)_{Y_{1}}\right)=\sum_{i=1}^{r} \alpha_{i} I\left(n f^{(1)}+X_{1}\left(f^{(1)}\right)_{X_{1}}, p_{i}\right) .
$$

Assim, é suficiente mostrar que para todo ramo plano $(p)$ de $\left(\left(f^{(1)}\right) Y_{1}\right)$ temos

$$
I\left(f^{(1)}, p\right)=I\left(n f^{(1)}+X_{1}\left(f^{(1)}\right)_{X_{1}}, p\right) .
$$

Suponhamos que $(p)$ pode ser parametrizada por $(\varphi, \psi), \varphi, \psi \in k[[T]]$. Então

$$
\frac{d}{d T} \varphi^{n} f^{(1)}(\varphi, \psi)=\varphi^{n-1}\left[n f^{(1)}(\varphi, \psi)+\varphi\left(f^{(1)}\right)_{X_{1}}(\varphi, \psi)\right] \frac{d \varphi}{d T}+\varphi^{n}\left(f^{(1)}\right)_{Y_{1}}(\varphi, \psi) \frac{d \psi}{d T},
$$

onde $\left(f^{(1)}\right)_{Y_{1}}(\varphi, \psi) \frac{d \psi}{d T}=p(\varphi, \psi) \frac{d \psi}{d T}=0$. Logo,

$$
\frac{d}{d T} \varphi^{n} f^{(1)}(\varphi, \psi)=\varphi^{n-1}\left[n f^{(1)}(\varphi, \psi)+\varphi\left(f^{(1)}\right)_{X_{1}}(\varphi, \psi)\right] \frac{d \varphi}{d T}
$$

Ainda, mult $\left(\frac{d}{d T}\left[\varphi^{n} f^{(1)}(\varphi, \psi)\right]\right)=\operatorname{nmult}(\varphi)+\operatorname{mult}\left(f^{(1)}(\varphi, \psi)\right)-1 \mathrm{e}$

$$
\begin{gathered}
\text { mult }\left(\varphi^{n-1}\left[n f^{(1)}(\varphi, \psi)+\varphi\left(f^{(1)}\right)_{X_{1}}(\varphi, \psi)\right] \frac{d \varphi}{d T}\right)= \\
(n-1) \text { mult }(\varphi)+\operatorname{mult}\left(n f^{(1)}(\varphi, \psi)+\varphi\left(f^{(1)}\right)_{X_{1}}(\varphi, \psi)\right)+\operatorname{mult}(\varphi)-1= \\
n \operatorname{mult}(\varphi)+\operatorname{mult}\left(n f^{(1)}(\varphi, \psi)+\varphi\left(f^{(1)}\right)_{X_{1}}(\varphi, \psi)\right)-1
\end{gathered}
$$

De (7.3) segue que

$$
n \text { mult }(\varphi)+\operatorname{mult}\left(f^{(1)}(\varphi, \psi)\right)-1=n \operatorname{mult}(\varphi)+\operatorname{mult}\left(n f^{(1)}(\varphi, \psi)+\varphi\left(f^{(1)}\right) X_{1}(\varphi, \psi)\right)-1
$$

Então

$$
\operatorname{mult}\left(f^{(1)}(\varphi, \psi)\right)=\operatorname{mult}\left(n f^{(1)}(\varphi, \psi)+\varphi\left(f^{(1)}\right) \dot{X}_{1}(\varphi, \psi)\right):
$$

Logo,

$$
I\left(f^{(1)}, p\right)=I\left(n f^{(1)}+X_{1}\left(f^{(1)}\right)_{X_{1}}, p\right)
$$

e o resultàdo s̈egue.

Teorema 7.4.5 (Milnor) Seja (f) um ramo plano. Se $\mu_{f}$ é o número de Milnor de $(f)$ e c é o condutor de $S(f)$, então $\mu_{f}=c$.

\section{Demonstração:}

Suponhamos que $f$ é irredutível de multiplicidade $n$ e regular em $Y$. Da definição de transformada estrita, vista no Capítulo 5, temos

$$
X_{1}^{n} f^{(1)}\left(X_{1}, Y_{1}\right)=f\left(X_{1}, X_{1} Y_{1}\right) .
$$

Derivando esta equação em relação a $Y_{1}$, obtemos

$$
X_{1}^{n}\left(f^{(1)}\right)_{Y_{1}}\left(X_{1}, Y_{1}\right)=f_{Y_{1}}\left(X_{1}, X_{1} Y_{1}\right) X_{1} .
$$


Portanto,

$$
\left(f^{(1)}\right)_{Y_{1}}=\frac{1}{X_{1}^{n-1}} f_{Y_{1}}\left(X_{1}, X_{1} Y_{1}\right)=\left(f_{Y}\right)^{(1)}
$$

Ainda, derivando a equação (7.4) em relação a $X_{1}$, obtemos

$$
\begin{aligned}
& n X_{1}^{n-1} f^{(1)}\left(X_{1}, Y_{1}\right)+X_{1}^{n}\left(f^{(1)}\right)_{X_{1}}\left(X_{1}, Y_{1}\right)=f_{X_{1}}\left(X_{1}, X_{1} Y_{1}\right)+Y_{1} f_{Y_{1}}\left(X_{1}, X_{1} Y_{1}\right) \\
& n X_{1}^{n-1} f^{(1)}\left(X_{1}, Y_{1}\right)+X_{1}^{n}\left(f^{(1)}\right)_{X_{1}}\left(X_{1}, Y_{1}\right)-Y_{1} f_{Y_{1}}\left(X_{1}, X_{1} Y_{1}\right)=f_{X_{1}}\left(X_{1}, X_{1} Y_{1}\right) .
\end{aligned}
$$

Se $g=f_{X}+f_{Y}$ então

$$
\begin{gathered}
g^{(1)}=\frac{1}{X_{1}^{n-1}}\left(f_{X_{1}}\left(X_{1}, X_{1} Y_{1}\right)+f_{Y_{1}}\left(X_{1}, X_{1} Y_{1}\right)\right)= \\
\frac{1}{X_{1}^{n-1}}\left(n X_{1}^{n-1} f^{(1)}\left(X_{1}, Y_{1}\right)+X_{1}^{n}\left(f^{(1)}\right)_{X_{1}}\left(X_{1}, Y_{1}\right)-Y_{1} f_{Y_{1}}\left(X_{1}, X_{1} Y_{1}\right)+f_{Y_{1}}\left(X_{1}, X_{1} Y_{1}\right)\right)= \\
n f^{(1)}\left(X_{1}, Y_{1}\right)+X_{1}\left(f^{(1)}\right)_{X_{1}}\left(X_{1}, Y_{1}\right)+\frac{\left(1-Y_{1}\right)}{X_{1}^{n-1}} f_{Y_{1}}\left(X_{1}, X_{1} Y_{1}\right)= \\
n f^{(1)}\left(X_{1}, Y_{1}\right)+X_{1}\left(f^{(1)}\right)_{X_{1}}\left(X_{1}, Y_{1}\right)+\left(1-Y_{1}\right)\left(f^{(1)}\right)_{Y_{1}}\left(X_{1}, Y_{1}\right) .
\end{gathered}
$$

Assim,

$$
\begin{gathered}
I\left(\left(f_{X}+f_{Y}\right)^{(1)}\left(X_{1}, Y_{1}\right),\left(f^{(1)}\right)_{Y_{1}}\left(X_{1}, Y_{1}\right)\right)= \\
I\left(n f^{(1)}\left(X_{1}, Y_{1}\right)+X_{1}\left(f^{(1)}\right)_{X_{1}}\left(X_{1}, Y_{1}\right)+\left(1-Y_{1}\right)\left(f^{(1)}\right)_{Y_{1}}\left(X_{1}, Y_{1}\right),\left(f^{(1)}\right)_{Y_{1}}\left(X_{1}, Y_{1}\right)\right)= \\
I\left(n f^{(1)}\left(X_{1}, Y_{1}\right)+X_{1}\left(f^{(1)}\right)_{X_{1}}\left(X_{1}, Y_{1}\right),\left(f^{(1)}\right)_{Y_{1}}\left(X_{1}, Y_{1}\right)\right) .
\end{gathered}
$$

Segue da Proposição 7.4.4 que

$$
I\left(\left(f_{X}+f_{Y}\right)^{(1)},\left(f^{(1)}\right)_{Y_{1}}\right)=I\left(n f^{(1)}+X_{1}\left(f^{(1)}\right)_{X_{1}},\left(f^{(1)}\right)_{Y_{1}}\right)=I\left(f^{(1)},\left(f^{(1)}\right)_{Y_{1}}\right) .
$$

Da Proposição 5.3.2, segue que

$$
I\left(f^{(1)},\left(f^{(1)}\right)_{Y_{1}}\right)=I\left(f^{(1)},\left(f_{Y}\right)^{(1)}\right)=I\left(f, f_{Y}\right)-\operatorname{mult}(f) \operatorname{mult}\left(f_{Y}\right) .
$$

Vimos que $I\left(f, f_{Y}\right)=c+n-1$ (ver Corolário 7.4.3), logo

$$
I\left(\left(f_{X}+f_{Y}\right)^{(1)},\left(f_{Y}\right)^{(1)}\right)=I\left(f^{(1)},\left(f^{(1)}\right)_{Y_{1}}\right)=c+n-1-n(n-1)=c-(n-1)^{2} .
$$

Também

$$
I\left(\left(f_{X}+f_{Y}\right)^{(1)},\left(f_{Y}\right)^{(1)}\right)=I\left(f_{X}+f_{Y}, f_{Y}\right)-\operatorname{mult}\left(f_{X}+f_{Y}\right) \text { mult }\left(f_{Y}\right)=I\left(f_{X}, f_{Y}\right)-(n-1)^{2} .
$$

Então, $\mu(f)=I\left(f_{X}, f_{Y}\right)=c$.

O Teorema acima mostra que o número de Milnor é um invariante da relação de equisingularidade de curvas e isto implica que $\mu$ é um invariante da relação de equivalência de curvas.

Nosso último teorema relaciona o semigrupo de um ramo plano com o semigrupo de sua transformada estrita.

Teorema 7.4.6 Suponhamos que $(f)$ é um ramo plano com cone tangente $\left(Y^{n}\right)$ e que $I(f, Y)=m$ não é divisivel por $n$. Seja $x_{0}, \ldots, x_{\tau}$ o sistema minimal de geradores de $S(f)$. Então o sistema minimal de geradores de $S\left(f^{(1)}\right)=S\left(\sigma^{*}(f)\right)$ é dado por 
(i) $x_{0}<x_{1}^{\prime}<\ldots<x_{r}^{\prime}$ se $x_{0}<x_{1}^{\prime}$

(ii) $x_{1}^{\prime}<x_{0}<x_{2}^{\prime}<\ldots<x_{r}^{\prime}$ se $x_{0}>x_{1}^{\prime}$ e $x_{1}^{\prime} \nmid x_{0}$,

(iii) $x_{1}^{\prime}<x_{2}^{\prime}<\ldots<x_{r}^{\prime}$ se $x_{0}>x_{1}^{\prime}$ e $x_{1}^{\prime} \mid x_{0}$.

onde $x_{j}^{\prime}=x_{j}-\frac{x_{0}^{2}}{e_{j-1}}$, onde $e_{j}=\operatorname{mdc}\left(x_{0}, \ldots, x_{j}\right), j=1, \ldots, r$.

\section{Demonstração:}

Do Teorema 7.3.3,

$$
x_{j}^{\prime}=a_{\frac{n}{e_{j-1}}}^{\prime}=a_{n_{1} n_{2} \ldots n_{j-1}}^{\prime}, j=1, \ldots, r .
$$

Mas, usando a Proposição 6.5.1 temos

$$
x_{j}^{\prime}=a_{n_{1} n_{2} \ldots n_{j-1}}^{\prime}=a_{n_{1} n_{2} \ldots n_{j-1}}-\left(n_{1} n_{2} \ldots n_{j-1}\right) n=x_{j}-\frac{n}{e_{j-1}} n=x_{j}-\frac{x_{0}^{2}}{e_{j-1}} .
$$

(i) Como $I(f, Y)=m$ então $I\left(\sigma^{*}(f), Y_{1}\right)=m-n($ ver $5.2 .3(i))$. Se $x_{0}<x_{1}^{\prime}$, ou seja, se $n<m-n$ então, da Proposição $5.2 .3(i i)$, mult $\left(\sigma^{*}(f)\right)=\operatorname{mult}(f)=n$ e $x_{0}^{\prime}=x_{0}$.

(ii) Se $m-n<n$ então mult $\left(\sigma^{*}(f)\right)=m-n<$ mult $(f)=n$. Então $x_{0}^{\prime}=m-n$ e $x_{1}^{\prime}=$ $\min \left(S\left(\sigma^{*}(f)\right) \backslash\langle m-n\rangle\right)$. Como $I\left(\sigma^{*}(f), X_{1}\right)=n$, então $n \in S\left(\sigma^{*}(f)\right)$. Temos que $n$ não é múltiplo de $m-n$ e' $n>m-n$. Além disso, $x_{2}^{\prime}=x_{2}-\frac{n^{2}}{e_{1}}>n$. Então $x_{1}^{\prime}=n$.

(ii) Se $x_{0}>x_{1}^{\prime}$ e $x_{1}^{\prime} \mid x_{0}$, então $x_{0}$ não pertence ao sistema minimal de geradores de $S\left(\sigma^{*}(f)\right)$. 


\section{Referências Bibliográficas}

[1] A.Hefez, Irreducible Plane Curve Singularities, Real and Complex Singularities, Lecture Notes Series in Pure and Applied Mathematics, Marcell-Decker, 2002, edited by D.Mond and M.J.Saia, $1-120$.

[2] M.F.Atiyah, I.G.MacDonald, Introduction to Commutative Algebra, Addison-Wesley, 1969.

[3] S.Lang, Algebra, Third Edition, Addison-Wesley, 1927.

[4] K.Hoffman, R.Kunze, Linear Algebra, Prentice-Hall, Inc, 1961.

[5] I.N.Herstein, Topics in Algebra, Blaisdell, New York, 1964.

[6] P.M.Cohn, Classic Algebra, John Wiley and Sons Ltd., 1981.

[7] J.M.Ruiz, The Basic Theory of Power Series, Advanced Lectures in Mathematics, Vieweg.

[8] O. Zariski, P.Samuel, Commutative Algebra, Volume 2, Van Nostrand, 1960.

[9] R.Walker, Algebraic Curves, Springer Verlag, 1978.

[10] W.Fulton, Algebraic Curves: an Introduction to Algebraic Geometry, Benjamin, New York, 1969.

[11] G.Angermüller, Die Wertehalbgruppe einer ebener irreduziblen algebroiden Kurve, Math. Zeitschr, 153:267-282, 1977

[12] J.Milnor, Singular Points of Complex Hypersurfaces, Annals of Mathematics Studies, 61, Princeton University Press, 1968.

[13] J.J.Risler, Sur L'idéal Jacobien D'une Courbe Plane, Bull. Soc. Math. France, 99:305-311, 1971. 
